\title{
Ti(OiPr)4-Enabled Dual Photoredox and Nickel-Catalyzed Arylation and Alkenylation of Cyclopropanols
}

\author{
Nastassia A. Varabyeva, Maryia V. Barysevich, Yauhen Aniskevich, Alaksiej Hurski
}

Submitted date: 11/05/2021 Posted date: 12/05/2021

Licence: CC BY-NC-ND 4.0

Citation information: Varabyeva, Nastassia A.; Barysevich, Maryia V.; Aniskevich, Yauhen; Hurski, Alaksiej (2021): Ti(OiPr)4-Enabled Dual Photoredox and Nickel-Catalyzed Arylation and Alkenylation of Cyclopropanols. ChemRxiv. Preprint. https://doi.org/10.26434/chemrxiv.14573148.v1

In this article, we report an application of cyclopropanols as coupling partners in the dual photoredox/nickel catalyzed reaction with aryl and vinyl bromides. We found that this cross-coupling proceeds smoothly in the presence of $\mathrm{Ti}(\mathrm{OiPr})_{4}$ as an additive to provide $\beta$-aryl or $\gamma, \delta$-unsaturated ketones.

File list (2)

Manuscript.pdf (0.96 MiB)

view on ChemRxiv - download file

SI.pdf (8.13 MiB)

view on ChemRxiv - download file 


\title{
$\operatorname{Ti}(\mathrm{O} i \mathrm{Pr})_{4}$-enabled dual photoredox and nickel-
}

\author{
catalyzed arylation and alkenylation of \\ cyclopropanols
}

Nastassia Varabyeva, ${ }^{a,{ }^{\dagger}}$ Maryia Barysevich, ${ }^{a, \dagger}$ Yauhen Aniskevich ${ }^{b}$ and Alaksiej Hurski ${ }^{a *}$

anstitute of Bioorganic Chemistry, National Academy of Sciences of Belarus, Kupreviča Str.

5/2, Minsk 220141, Belarus; ${ }^{b}$ Belarusian State University, Niezaliežnasci Av. 4, Minsk 220030, Belarus

KEYWORDS cyclopropanol, photoredox, Ni-catalyst, radical, ring-opening

ABSTRACT. Readily available from esters and kektones, cyclopropanols are inclined to undergo diverse ring-opening reactions. Their one-electron oxidation is a conventional way to $\beta$-carbonyl radicals. However, despite this fact, their application as a coupling partner in dual photoredox and nickel-catalyzed reactions with organic halides remains underdeveloped. Here, we report that $\mathrm{Ti}(\mathrm{O} i \mathrm{Pr})_{4}$ additive enables this elusive cross-coupling with aryl and alkenyl bromides affording $\beta$ substituted ketones. 
Nickel-catalyzed coupling of the photochemically generated alkyl radicals ${ }^{1}$ with aryl, alkenyl or alkyl halides has recently emerged as a powerful tool for the C-C bond construction (Scheme 1A). ${ }^{2}$ Proceeding under mild conditions, these reactions generally tolerate sensitive functional groups and moreover, can be deployed for the formation of an asymmetric setereocenter. ${ }^{3}$ The scope of the radical precursors used in the coupling is exceptionally broad. After the pioneering reports on the arylation of organotrifluoroborates, aliphatic carboxylic acids and $\mathrm{N}, \mathrm{N}$-dimethylaniline by Molander ${ }^{4}$ and MacMillan and Doyle, ${ }^{5}$ various alternative coupling partners were utilized including alkylsylicates, ${ }^{6}$ monoalkyl oxalates, ${ }^{7}$ dihydropyridines, ${ }^{8},{ }^{9}$ alkyl halides,${ }^{10}$ alkanes, ${ }^{11}$ oxiranes, ${ }^{12}$ aziridines, ${ }^{13}$ cycloalkanone oxyme-carboxylates, ${ }^{14}$ N-hydroxyphthalimide esters, ${ }^{15}$ Katritzky salts, ${ }^{16}$ alkylsulfinate salts, ${ }^{17}$ xanthate esters, ${ }^{18}$ boracene-based alkyl borates, ${ }^{19}$ linear ${ }^{20}$ and cyclic alcohols. ${ }^{21}$ Being a source of $\beta$-ketoradicals $\mathbf{3},{ }^{22}$ cyclopropanols $\mathbf{1}$ can also undergo the photoredox/nickel dual catalyzed reaction to provide $\beta$-substituted ketones (Scheme 1B). ${ }^{21}$ However, scope of cyclopropanols that can be engaged in this coupling is rather narrow. Shenvi reported arylation and alkenylation of tricyclic silyloxycyclopropanes $\mathbf{4}$ promoted by an iridium photocatalyst and a nickel complex. ${ }^{21 a}$ One of the obtained $\beta$-substituted ketones $\mathbf{5}$ was further efficiently applied in a concise synthesis of natural alkaloid GB-22. ${ }^{21 a}$ Another example of the photoredox initiated ring-opening arylation was described by Rueping. ${ }^{21 b}$ The presence of PMP group in $\mathbf{6}$ was crucial because the formation of alkoxy radical $\mathbf{2}$ was initiated by the one-electron oxidation of this moiety. Here, we report that limitations in the scope of photoredox and nickel dual-catalyzed cyclopropanol arylation and alkenylation can be overcome when the reaction is carried out in the presence of $\operatorname{Ti}(\mathrm{O} i \mathrm{Pr})_{4}$ as an additive. Under these conditions, $\beta$-substituted ketones $\mathbf{1 0}$ can be obtained from cyclopropanols $\mathbf{8}$ and aryl- or vinyl- bromides $\mathbf{9}$. 

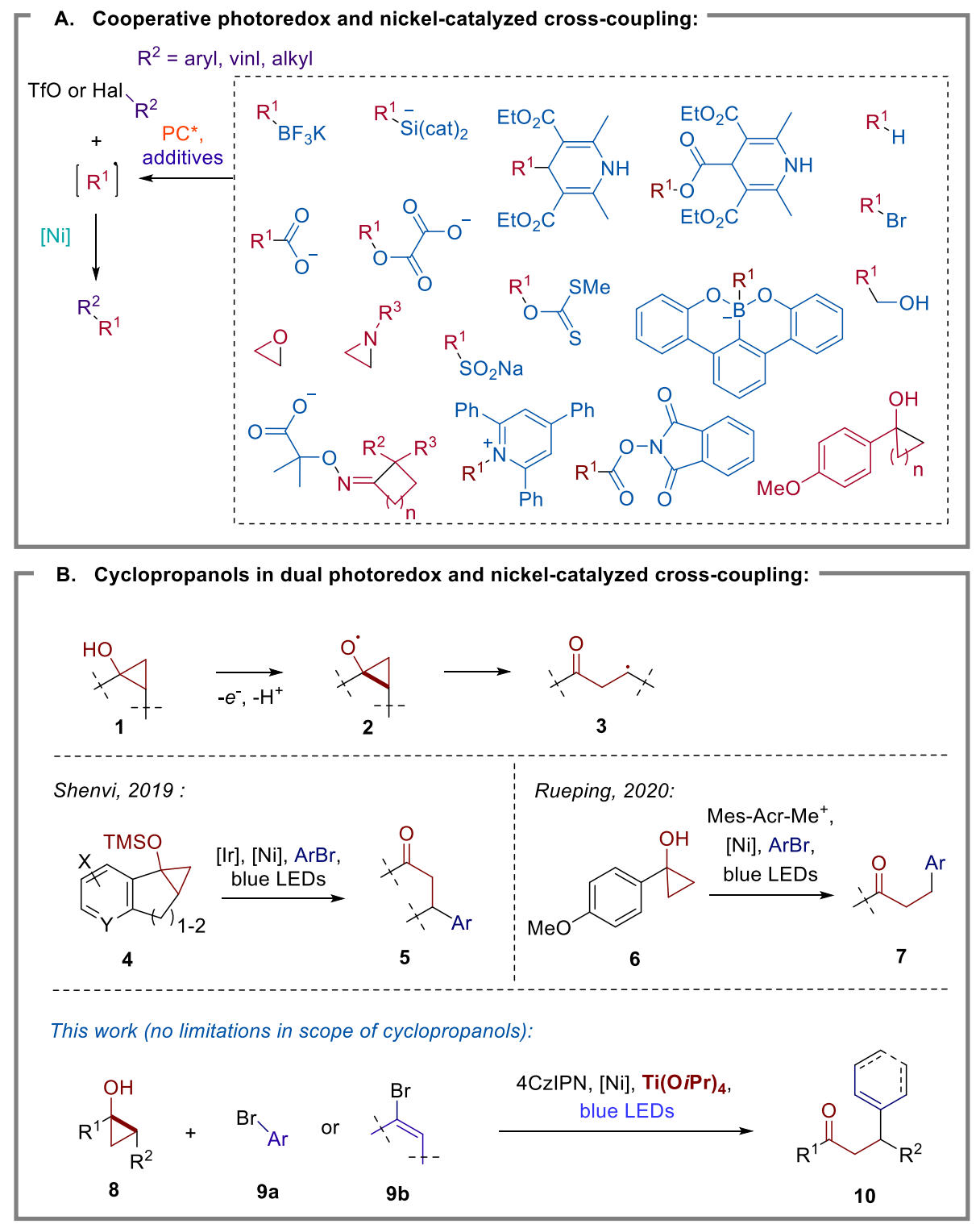

Scheme 1. Reactions of cyclopropanols under dual photoredox and nickel catalysis

Transformation of cyclopropanol $\mathbf{1}\left(\mathrm{E}^{\mathrm{ox}}=+1.66 \mathrm{~V}\right)^{23}$ to oxycyclopropyl radical $\mathbf{2}$ require a relatively strong oxidant. For comparison, the reduction potential of the excited photocatalysts commonly used in the cooperative photoredox and nickel catalysis lays between +0.77 and +1.35 $\mathrm{V}^{2 \mathrm{c}}$ Thus, either a photocatalyst with a stronger oxidative properties or an alternative way of oxycyclopropyl radical 2 genertion was required. Recently we found that cyclopropanols undergo 
one-electron oxidation by the photoexcited acridimium salts $\left(\mathrm{E}_{1 / 2}\left(\mathrm{P}^{*} / \mathrm{P}^{-}\right)=+2.08 \mathrm{~V}\right),{ }^{24}$ but our attempts to use them in the ring-opening arylation of $\mathbf{8}$ with 9 were unsuccessful. Nevertheless, we found that addition of $\mathrm{Ti}(\mathrm{O} i \mathrm{Pr})_{4}$ to the reaction mixture enables the reaction even when $4 \mathrm{CzIPN}$ $\left(\mathrm{E}_{1 / 2}\left(\mathrm{P}^{*} / \mathrm{P}^{-}\right)=+1.35 \mathrm{~V}\right)^{25}$ is employed as a photocatalyst. The highest yield in the arylation of 11a with $p$-bromoanisol (12a) was achieved when the reaction was run in acetone in the presence of the photocatalyst, nickel chloride bipyridine complex, potassium carbonate as a base and titanium isopropoxide as an additive. Changing the solvent with acetonitrile and DMA resulted in a slight drop in yield, but in THF, the arylation was significantly less efficient. When di-tertbutylbipyridine, batophenanthrolin, neocuproine or dimethoxybipyridine were used as alternative ligands, the yield of 13a diminished. While inorganic salt $\mathrm{K}_{3} \mathrm{PO}_{4}$ can be employed as a base of choice, the reaction in the presence of 2,6-lutidine proceeded giving the product in a low $23 \%$ yield. The yield of $\mathbf{1 0 a}$ in the reaction promoted by $\mathrm{Ti}(\mathrm{O} t \mathrm{Bu})_{4}$ additive with bulk tert-butoxide ligands was slightly lower than that under the standard conditions. Trimethyl borate also promoted the cross-coupling, though, significantly less efficiently. No reaction was observed when aluminum isopropoxide was used as an additive or when the arylation was carried out in the absence of $\mathrm{Ti}(\mathrm{O} i \mathrm{Pr})_{4}$ or a photocatalyst.

Table 1. Optimization of the reaction conditions. ${ }^{a}$

\begin{tabular}{lcc}
\hline reaction conditions & yield of 13a $(\%))^{b}$ \\
\hline 1 & Standard conditions & $60 \%$ \\
2 eq. $\mathrm{Ti}(\mathrm{O} / \mathrm{Pr})_{4}, 3 \mathrm{eq} \cdot \mathrm{K}_{2} \mathrm{CO}_{3}$, \\
acetone, blue $\mathrm{LEDs}, 15 \mathrm{~h}$
\end{tabular}




$\begin{array}{ccc}3 & \mathrm{MeCN} \text { instead of acetone } & 49 \% \\ 4 & \mathrm{DMA} \text { instead of acetone } & 51 \% \\ 5 & \mathrm{NiCl}_{2} \cdot \mathrm{DME} \text { and dtbbpy instead of } \mathrm{NiCl}_{2} \cdot \text { bpy } & 47 \% \\ 6 & \mathrm{BPhen} \cdot \mathrm{NiCl}_{2} \cdot 2 \mathrm{DMF} \text { instead of } \mathrm{NiCl}_{2} \cdot \text { bpy } & 47 \% \\ 7 & \mathrm{NiCl}_{2} \cdot \mathrm{DME} \text { and neocuproine instead of } \mathrm{NiCl}_{2} \cdot \text { bpy } & 21 \% \\ 8 & \mathrm{NiCl}_{2} \cdot \mathrm{DME} \text { and dMeObpy instead of } \mathrm{NiCl}_{2} \cdot \text { bpy } & 30 \% \\ 9 & \mathrm{~K}_{3} \mathrm{PO}_{4} \text { instead of } \mathrm{K}_{2} \mathrm{CO}_{3} & 56 \% \\ 10 & 2,6-\mathrm{lutidine}_{2} \text { instead of } \mathrm{K}_{2} \mathrm{CO}_{3} & 23 \% \\ 11 & \mathrm{Ti}(\mathrm{O} t \mathrm{Bu})_{4} \text { instead of } \mathrm{Ti}(\mathrm{O} i \mathrm{Pr})_{4} & 47 \% \\ 12 & \mathrm{~B}(\mathrm{OMe})_{3} \text { instead of } \mathrm{Ti}(\mathrm{O} i \mathrm{Pr})_{4} & 19 \% \\ 13 & \mathrm{Al}(\mathrm{O} i \mathrm{Pr})_{3} \text { instead of } \mathrm{Ti}(\mathrm{O} i \mathrm{Pr})_{4} \text { or no } \mathrm{Ti}(\mathrm{O} i \mathrm{Pr})_{4} \text { or no } 4 \mathrm{CzIPN} & 0 \%\end{array}$

\begin{abstract}
${ }^{a}$ Reaction conditions: 11a $(0.1 \mathrm{mmol}), 12 \mathrm{a}(0.2 \mathrm{mmol})$, photocatalyst $(0.005 \mathrm{mmol}), \mathrm{NiCl}_{2} \cdot \mathrm{bpy}$ $(0.005 \mathrm{mmol})$ or $\mathrm{NiCl}_{2} \cdot \mathrm{DME}(0.005 \mathrm{mmol})$, ligand $(0.005 \mathrm{mmol})$, additive $(0.2 \mathrm{mmol})$, base $(0.3$ mmol) and solvent $(1 \mathrm{~mL})$, blue LEDs, $15 \mathrm{~h} .{ }^{\mathrm{b}} \mathrm{Crude}{ }^{1} \mathrm{H}$ NMR yield with $\mathrm{CH}_{2} \mathrm{Br}_{2}$ as internal standard; 4CzIPN = 2,4,5,6-Tetra(9H-carbazol-9-yl)isophthalonitrile; bpy $=$ 2,2'-bipyridine; dtbbpy $=4,4^{\prime}$-di-tert-butyl-2,2'-bipyridine; BPhen $=4,4^{\prime}$-diphenyl-2,2'-bipyridine; DME $=1,2$ dimethoxyethane; DMA = N,N-dimethylacetamide; dMeObpy = 4,4'-dimethoxy-2,2'-bipyridine.
\end{abstract}

Having optimized the reaction conditions, we next investigated the scope of the reaction. Aryl bromides with donor or acceptor functional groups, as well as an ortho-substituent reacted with 11a giving desired $\beta$-arylketones 13a-f. Silylated hydroxyl group, alkenyl and diethylacetal units in the cyclopropanol substrate were tolerated but formation of $\mathbf{1 3 h}$ and $13 \mathbf{i}$ was slightly less efficient. The reaction of the 1,2-disubstituted cyclopropanol afforded arylketone $\mathbf{1 3} \mathbf{j}$ as a single $\beta$-branched regioisomer. In contrast to the palladium-catalysed arylation which leads to $\alpha$-branched products, ${ }^{26}$ the investigated radical reaction proceeded with cleavage of the more substituted bond of the three-carbon ring.

Next, cross-coupling of cyclopropanols with vinyl halides was investigated. Generally, yields of the alkenylation were better when the reaction was carried out in the presence of neocuproine instead of bipyridine ligand. The reaction between cyclopropanol 11a and 2-bromoalkenes 
provided ketones 14a and 14b in $84 \%$ and $55 \%$ yields, respectively. Alkenylation of 11a with vinyl triflate also proceeded efficiently to produce $\beta$-cyclohexenylketone $14 \mathbf{c}$. In synthesis of $\gamma, \delta$ unsturated ketones 14d and 14e from 2-alkylsubstituted vinyl bromides and cyclopropanol 11a, bipyridine ligand was more favorable than neocuproine and the products were isolated in $45 \%$ and $46 \%$ yields, respectively. Formation of Z-alkenylketone $14 \mathrm{e}$ was accompanied by a little isomerization of the double bond, which caused drop in $Z / E$ ratio to $8: 1$. Diminishing of the isomeric purity was more significant for the products $14 \mathrm{~g}$ and $14 \mathrm{~h}$ bearing the alkene unit conjugated with an electron-rich aromatic ring. After completion of the coupling, $\mathbf{1 4 g}$ was isolated as a $6: 1$ mixture of $E / Z$ stereoisomers. This ratio further dropped to $1.7: 1$ when the reaction time was increased to $48 \mathrm{~h}$. Ketone product $\mathbf{1 4 f}$ with the unsubstituted benzene ring was obtained as a single $E$-isomer. Aryl chloride units were found to be inert under the reaction conditions and ketone 14h was prepared from the corresponding vinyl bromide in a good $61 \%$ yield. Then, diverse 1mono and 1,2-disubstituted cyclopropanols were tested in the reaction with 2-bromopropene. The substrate bearing two hydroxycyclopropyl groups underwent smooth coupling giving diketone 14i in 51\% yield. Silylated and unprotected hydroxyl groups, alkenyl unit and acetal protecting groups were tolerated and corresponding products $\mathbf{1 4} \mathbf{j}-\mathbf{n}$ were isolated in $41-75 \%$ yields. The reaction conditions were mild enough for the preparation of enantiomerically pure alkenylketones 140-p that contain sensitive $\alpha$-stereocenters. Alkenylation of 1,2-disubstituted cyclopropanols provided regioisomerically pure $\beta$-branched products $\mathbf{1 4 q - s}$ in $41-64 \%$ yields. These reactions proceeded more efficiently in the presence of bipyridine ligand instead of neocuproine. Finally, $\beta$ isopropenylcycloheptanone $\mathbf{1 4 t}$ was obtained from the bicyclic cyclopropanol in $57 \%$ yield. 
Table 2. The scope of the reaction ${ }^{a, b}$
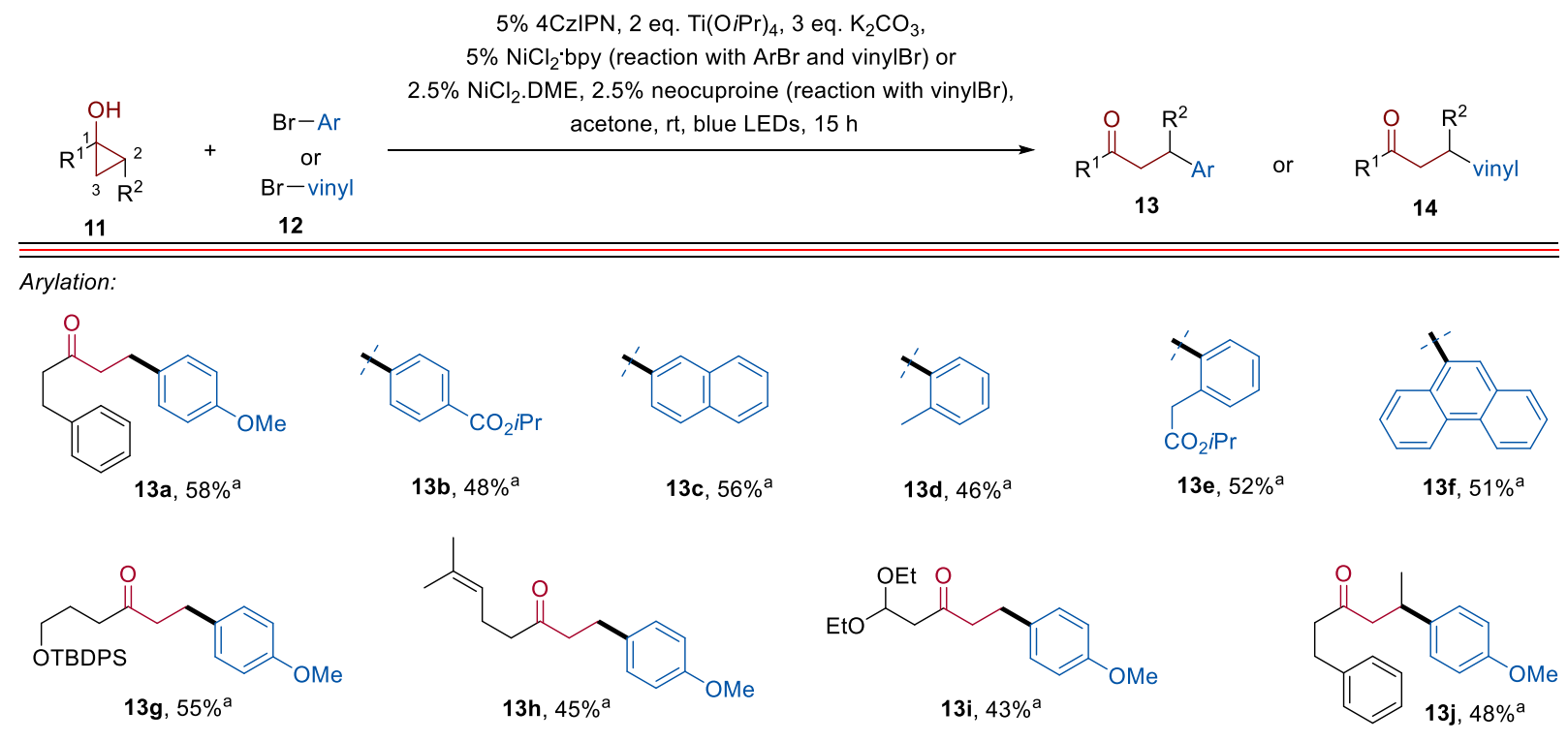

Alkenylation:<smiles>C=C(C)CCC(=O)CCc1ccccc1</smiles>

$14 \mathrm{a}, 84 \%^{\mathrm{b}}$

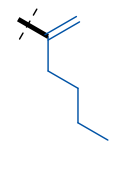

$14 b, 55 \%$ b
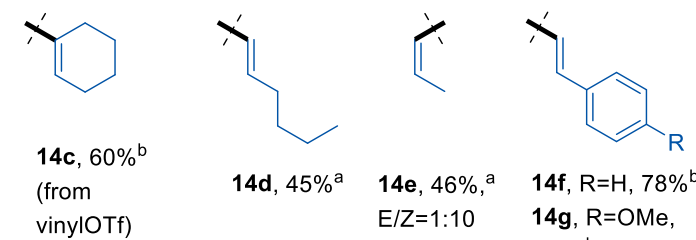

vinylOTf)

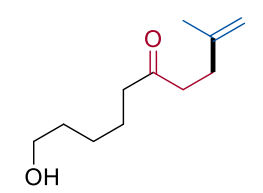

$14 k, 67 \%^{b}$

$14 \mathrm{j}, 75 \%^{\mathrm{b}}$

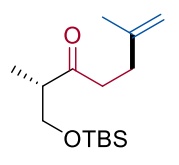

$14 p, 55 \%{ }^{b}$

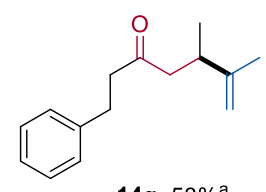

$14 q, 53 \%^{a}$

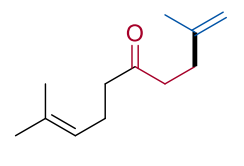

$141,65 \%^{b}$
$14 \mathrm{~g}, \mathrm{R}=\mathrm{OMe}$, $41 \%,{ }^{b} E / Z=6: 1$

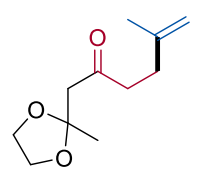

$14 \mathrm{~m}, 41 \%^{\mathrm{b}}$<smiles>C=C(C)C(CCCC[SeH2+][SbH2])CC(=O)CCc1ccccc1</smiles>

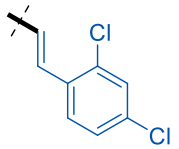

14h, $61 \%$, $E / Z=6: 1$

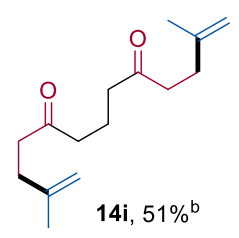<smiles>C=C(C)CCC(=O)CC(OCC)OCC</smiles>

$14 \mathrm{n}, 65 \%^{\mathrm{b}}$

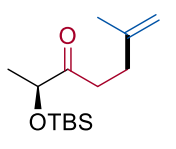

$140,48 \%{ }^{b}$<smiles>C=C(C)C(CC(=O)CCc1ccccc1)CC(CC)O[Mg]</smiles><smiles>C=C(C)C1CCc2cc(OC)ccc2C(=O)C1</smiles>

${ }^{a}$ Reaction conditions A: $11(0.25 \mathrm{mmol}), 12(0.5 \mathrm{mmol}), 4 \mathrm{CzIPN}(0.0125 \mathrm{mmol}), \mathrm{NiCl}_{2} \cdot \mathrm{bpy}$ (0.0125 mmol), $\mathrm{Ti}(\mathrm{O} i \mathrm{Pr})_{4}(0.5 \mathrm{mmol}), \mathrm{K}_{2} \mathrm{CO}_{3}(0.75 \mathrm{mmol})$, acetone $(2.5 \mathrm{~mL})$, blue LEDs, $15 \mathrm{~h}$; ${ }^{b}$ Reaction conditions $\mathrm{B}$ : the same as reaction conditions $\mathrm{A}$, but $\mathrm{NiCl}_{2} \cdot \mathrm{DME}(0.00625 \mathrm{mmol})$ and neocuproine $(0.00625 \mathrm{mmol})$ were used instead of $\mathrm{NiCl}_{2}$ bpy $(0.0125 \mathrm{mmol})$. 
To get insight into the role of $\mathrm{Ti}(\mathrm{O} i \mathrm{Pr})_{4}$ in the ring-opening cross-coupling, additional control experiments were carried out (Scheme 2A). First, the reaction between cyclopropane 11a and $\operatorname{Ti}(\mathrm{O} i \mathrm{Pr})_{4}$ in acetone- $d^{6}$ was investigated. We observed a fast exchange between the isopropoxyde and cyclopropyloxy ligands leading to 1:3 mixture of 11a and 15. Initially, we assumed that the formed titanium cyclopropoxide complex $\mathbf{1 5}$ could undergo one-electron oxidation by the photoexcited 4CzIPN* more efficiently than free cyclopropanol 11a. However, voltamperograms of 11a and its 1:1 mixture with $\operatorname{Ti}(\mathrm{O} i \mathrm{Pr})_{4}$ were nearly identical suggesting that the additive played another role in the reaction. Alternatively, $\mathrm{Ti}(\mathrm{O} i \mathrm{Pr})_{4}$ could serve as a co-catalyst that assists formation of cyclopropyloxy nickel(III) complexes. ${ }^{27}$ Homolytic cleavage of RO-Ni ${ }^{\mathrm{III}}$ bond could provide cyclopropyloxy radicals, which could further undergo the ring-opening and crosscoupling. ${ }^{28,29,30}$. Bering in mind that formation of $\mathrm{RO}-\mathrm{Ni}^{\mathrm{III}}$ intermediates is possible even in the absence of a photocatalyst and this reaction proceeds more efficiently under the irradiation with 390-395 nm LEDs, ${ }^{27 \mathrm{e}}$ we carried out additional control experiments without 4CzIPN. When blue LEDs were used as a source of light, the alkenylation of 11a by 2-bromopropene gave product 14a in $10 \%$ yield, while no reaction was observed in the absence of $\mathrm{Ti}(\mathrm{O} i \mathrm{Pr})_{4}$. Under the irradiation with purple LEDs, the yield increased significantly to $38 \%$ and $1 \%$ of the product was formed in the absence of $\operatorname{Ti}(\mathrm{O} i \mathrm{Pr})_{4}$. Based on these experiments, we proposed a catalytic cycle in which 4CzIPN* oxidizes the nickel complex rather than cyclopropanol or its titanium alkoxide while $\mathrm{Ti}(\mathrm{O} i \mathrm{Pr})_{4}$ assists the formation of $\mathrm{RO}-\mathrm{Ni}^{\mathrm{III}}$ intermedates (Scheme 2B). After the oxidative addition of aryl or vinyl bromide to $\mathbf{1 6}$, aryl nickel complex $\mathbf{1 7}$ would undergo oxidation by the photoexcited $4 \mathrm{CzIPN} *$ and ligand exchange with titanium alkoxide $\mathbf{1 8}$ to provide $\mathrm{Ni}^{\mathrm{III}}$ complex 19. Next, $\mathrm{Ni}^{\mathrm{III}}-\mathrm{O}$ bond in $\mathbf{1 9}$ would break providing $\mathrm{Ni}^{\mathrm{II}}$ complex $\mathbf{2 0}$ and oxycyclopropyl radical 21. Ring-opening of $\mathbf{2 1}$ would give $\beta$-ketoradical $\mathbf{2 2}$ which would further react with $\mathbf{2 0}$ affording 
$\mathrm{Ni}^{\mathrm{III}}$ complex 23. Reductive elimination from $\mathbf{2 3}$ would lead to coupling product $\mathbf{2 4}$ and $\mathrm{Ni}^{\mathrm{I}}$ species 25, reduction of which would close the catalytic cycle.
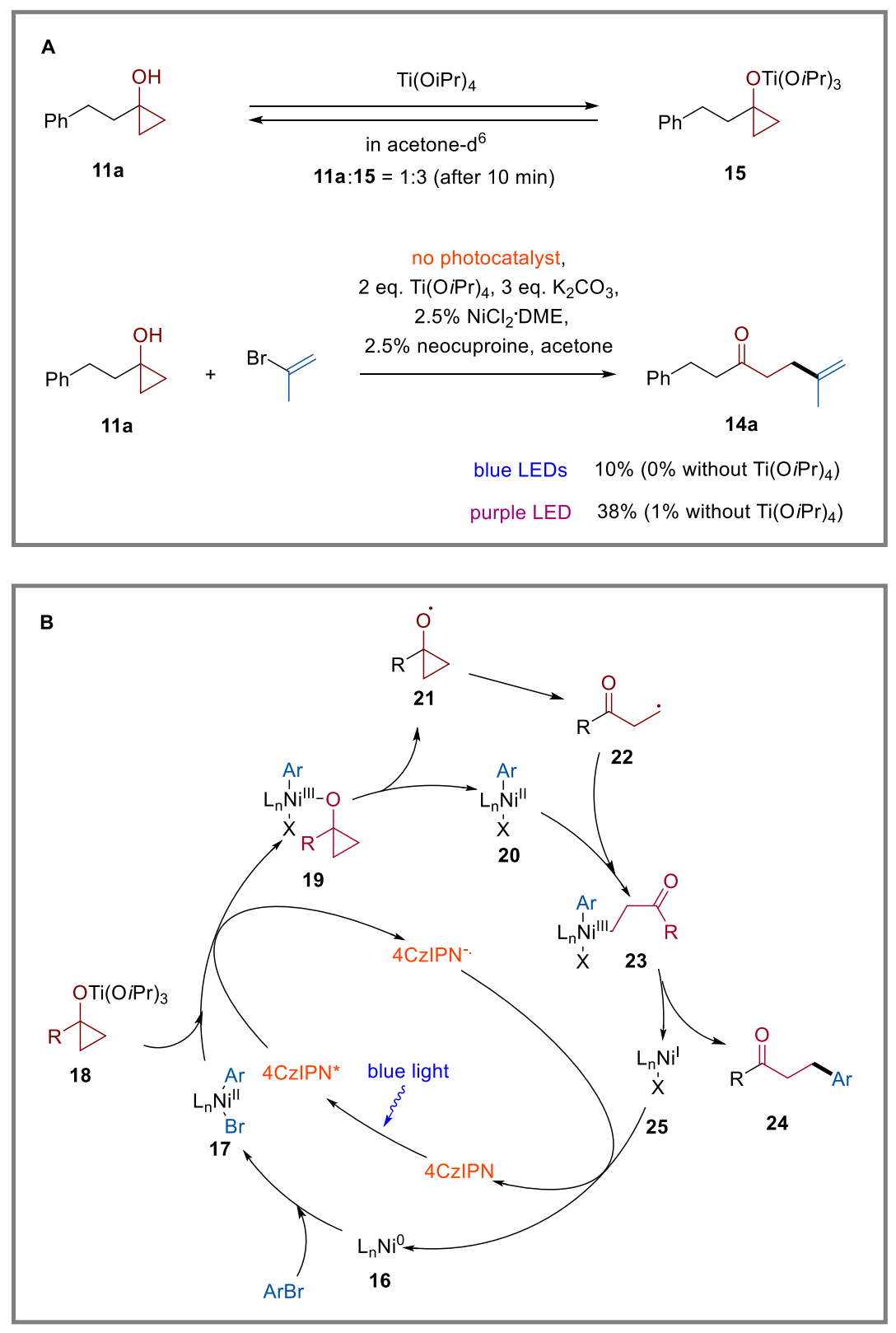

Scheme 2. Control experiments and plausible catalytic cycle

In conclusion, we have developed a general approach to $\beta$-aryl and $\beta$-alkenylketones from cyclopropanols and aryl or alkenyl bromides or triflates. We found, that this photoredox and nickel dual catalyzed reaction becomes general for a broad scope of 1-mono- and 1,2-disubstituted 
cyclopropanols when carried out in the presence of $\operatorname{Ti}(\mathrm{O} i \mathrm{Pr})_{4}$ additive. The cross-coupling is compatible with functional groups including unprotected hydroxyl and proceeds under conditions mild enough for the preparation of enantiomerically pure ketones bearing a sensitive $\alpha$ stereocenter.

\section{ASSOCIATED CONTENT}

\section{Supporting Information.}

Experimental procedures and characterization of all new compounds (PDF)

\section{AUTHOR INFORMATION}

\section{Corresponding Author}

Alaksiej Hurski - Institute of Bioorganic Chemistry, National Academy of Sciences of Belarus, Kupreviča Str. 5/2, Minsk 220141, Belarus; https://orcid.org/0000-0002-8599-2621; Email: AHurski@iboch.by

\section{Author Contributions}

$\dagger$ These authors contributed equally.

\section{Notes}

The authors declare no competing financial interest.

\section{ACKNOWLEDGMENT}

We are grateful for the financial support from the Belarusian Foundation for Fundamental Research (projects X20M-036). 


\section{REFERENCES}

1. Crespi, S.; Fagnoni, M., Generation of Alkyl Radicals: From the Tyranny of Tin to the Photon Democracy. Chem. Rev. 2020, 120, 9790-9833.

2. (a) Skubi, K. L.; Blum, T. R.; Yoon, T. P., Dual Catalysis Strategies in Photochemical Synthesis. Chem. Rev. 2016, 116, 10035-10074; (b) Twilton, J.; Le, C.; Zhang, P.; Shaw, M. H.; Evans, R. W.; MacMillan, D. W. C., The merger of transition metal and photocatalysis. Nature Reviews Chemistry 2017, 1, 0052; (c) Milligan, J. A.; Phelan, J. P.; Badir, S. O.; Molander, G. A., Alkyl Carbon-Carbon Bond Formation by Nickel/Photoredox Cross-Coupling. Angew. Chem. Int. Ed. 2019, 58, 6152-6163; (d) Matsui, J. K.; Lang, S. B.; Heitz, D. R.; Molander, G. A., PhotoredoxMediated Routes to Radicals: The Value of Catalytic Radical Generation in Synthetic Methods Development. ACS Catal. 2017, 7, 2563-2575; (e) Cheng, W.-M.; Shang, R., Transition MetalCatalyzed Organic Reactions under Visible Light: Recent Developments and Future Perspectives. ACS Catal. 2020, 10, 9170-9196; (f) Haibach, M. C.; Ickes, A. R.; Wilders, A. M.; Shekhar, S., Recent Advances in Nonprecious Metal Catalysis. Org. Proc. Res. Dev. 2020, 24, 2428-2444; (g) Zheng, S.; Hu, Y.; Yuan, W., Recent Advances in C(sp3)-C(sp3) Cross-Coupling via Metalla-photoredox Strategies. Synthesis 2021, 53, 1719-1733.

3. Lipp, A.; Badir, S. O.; Molander, G. A., Stereoinduction in Metallaphotoredox Catalysis. Angew. Chem. Int. Ed. 2021, 60, 1714-1726.

4. (a) Tellis, J. C.; Primer, D. N.; Molander, G. A., Single-electron transmetalation in organoboron cross-coupling by photoredox/nickel dual catalysis. Science 2014, 345, 433; (b) Tellis, J. C.; Kelly, C. B.; Primer, D. N.; Jouffroy, M.; Patel, N. R.; Molander, G. A., SingleElectron Transmetalation via Photoredox/Nickel Dual Catalysis: Unlocking a New Paradigm for sp3-sp2 Cross-Coupling. Acc. Chem. Res. 2016, 49, 1429-1439; (c) Primer, D. N.; Molander, G. A., Enabling the Cross-Coupling of Tertiary Organoboron Nucleophiles through RadicalMediated Alkyl Transfer. J. Am. Chem. Soc. 2017, 139, 9847-9850; (d) Yu, X.-Y.; Zhou, Q.-Q.; Wang, P.-Z.; Liao, C.-M.; Chen, J.-R.; Xiao, W.-J., Dual Photoredox/Nickel-Catalyzed Regioselective Cross-Coupling of 2-Arylaziridines and Potassium Benzyltrifluoroborates: Synthesis of $\beta$-Substitued Amines. Org. Lett. 2018, 20, 421-424; (e) Campbell, M. W.; Compton, J. S.; Kelly, C. B.; Molander, G. A., Three-Component Olefin Dicarbofunctionalization Enabled by Nickel/Photoredox Dual Catalysis. J. Am. Chem. Soc. 2019, 141, 20069-20078.

5. (a) Zuo, Z.; Ahneman, D. T.; Chu, L.; Terrett, J. A.; Doyle, A. G.; MacMillan, D. W. C., Merging photoredox with nickel catalysis: Coupling of $\alpha$-carboxyl $\mathrm{sp}<\sup >3</$ sup $>$-carbons with aryl halides. Science 2014, 345, 437-440; (b) Noble, A.; MacMillan, D. W. C., Photoredox $\alpha-$ Vinylation of $\alpha$-Amino Acids and N-Aryl Amines. J. Am. Chem. Soc. 2014, 136, 11602-11605; (c) Zuo, Z.; Cong, H.; Li, W.; Choi, J.; Fu, G. C.; MacMillan, D. W. C., Enantioselective Decarboxylative Arylation of $\alpha$-Amino Acids via the Merger of Photoredox and Nickel Catalysis. J. Am. Chem. Soc. 2016, 138, 1832-1835; (d) Johnston, C. P.; Smith, R. T.; Allmendinger, S.; MacMillan, D. W. C., Metallaphotoredox-catalysed sp3-sp3 cross-coupling of carboxylic acids with alkyl halides. Nature 2016, 536, 322-325; (e) Kölmel, D. K.; Meng, J.; Tsai, M.-H.; Que, J.; Loach, R. P.; Knauber, T.; Wan, J.; Flanagan, M. E., On-DNA Decarboxylative Arylation: Merging Photoredox with Nickel Catalysis in Water. ACS Combinatorial Science 2019, 21, 588597. 
6. (a) Corcé, V.; Chamoreau, L.-M.; Derat, E.; Goddard, J.-P.; Ollivier, C.; Fensterbank, L., Silicates as Latent Alkyl Radical Precursors: Visible-Light Photocatalytic Oxidation of Hypervalent Bis-Catecholato Silicon Compounds. Angew. Chem. Int. Ed. 2015, 54, 11414-11418; (b) Jouffroy, M.; Primer, D. N.; Molander, G. A., Base-Free Photoredox/Nickel Dual-Catalytic Cross-Coupling of Ammonium Alkylsilicates. J. Am. Chem. Soc. 2016, 138, 475-478; (c) Lévêque, C.; Chenneberg, L.; Corcé, V.; Goddard, J.-P.; Ollivier, C.; Fensterbank, L., Primary alkyl biscatecholato silicates in dual photoredox/nickel catalysis: aryl- and heteroaryl-alkyl cross coupling reactions. Org. Chem. Front. 2016, 3, 462-465; (d) Patel, N. R.; Molander, G. A., Phenol Derivatives as Coupling Partners with Alkylsilicates in Photoredox/Nickel Dual Catalysis. J. Org. Chem. 2016, 81, 7271-7275; (e) Patel, N. R.; Kelly, C. B.; Jouffroy, M.; Molander, G. A., Engaging Alkenyl Halides with Alkylsilicates via Photoredox Dual Catalysis. Org. Lett. 2016, 18, 764-767; (f) Jouffroy, M.; Davies, G. H. M.; Molander, G. A., Accessing Elaborated 2,1Borazaronaphthalene Cores Using Photoredox/Nickel Dual-Catalytic Functionalization. Org. Lett. 2016, 18, 1606-1609; (g) Lévêque, C.; Corcé, V.; Chenneberg, L.; Ollivier, C.; Fensterbank, L., Photoredox/Nickel Dual Catalysis for the C(sp3)-C(sp3) Cross-Coupling of Alkylsilicates with Alkyl Halides. Eur. J. Org. Chem. 2017, 2017, 2118-2121; (h) Remeur, C.; Kelly, C. B.; Patel, N. R.; Molander, G. A., Aminomethylation of Aryl Halides Using $\alpha$-Silylamines Enabled by Ni/Photoredox Dual Catalysis. ACS Catal. 2017, 7, 6065-6069; (i) Lin, K.; Wiles, R. J.; Kelly, C. B.; Davies, G. H. M.; Molander, G. A., Haloselective Cross-Coupling via Ni/Photoredox Dual Catalysis. ACS Catal. 2017, 7, 5129-5133; (j) Vara, B. A.; Jouffroy, M.; Molander, G. A., C(sp3)$\mathrm{C}(\mathrm{sp} 2)$ cross-coupling of alkylsilicates with borylated aryl bromides - an iterative platform to alkylated aryl- and heteroaryl boronates. Chem. Sci. 2017, 8, 530-535; (k) García-Domínguez, A.; Mondal, R.; Nevado, C., Dual Photoredox/Nickel-Catalyzed Three-Component Carbofunctionalization of Alkenes. Angew. Chem. Int. Ed. 2019, 58, 12286-12290; (1) Levernier, E.; Corcé, V.; Rakotoarison, L.-M.; Smith, A.; Zhang, M.; Ognier, S.; Tatoulian, M.; Ollivier, C.; Fensterbank, L., Cross coupling of alkylsilicates with acyl chlorides via photoredox/nickel dual catalysis: a new synthesis method for ketones. Org. Chem. Front. 2019, 6, 1378-1382.

7. Zhang, X.; MacMillan, D. W. C., Alcohols as Latent Coupling Fragments for Metallaphotoredox Catalysis: sp3-sp2 Cross-Coupling of Oxalates with Aryl Halides. J. Am. Chem. Soc. 2016, 138, 13862-13865.

8. (a) Gutiérrez-Bonet, Á.; Tellis, J. C.; Matsui, J. K.; Vara, B. A.; Molander, G. A., 1,4Dihydropyridines as Alkyl Radical Precursors: Introducing the Aldehyde Feedstock to Nickel/Photoredox Dual Catalysis. ACS Catal. 2016, 6, 8004-8008; (b) Buzzetti, L.; Prieto, A.; Roy, S. R.; Melchiorre, P., Radical-Based C-C Bond-Forming Processes Enabled by the Photoexcitation of 4-Alkyl-1,4-dihydropyridines. Angew. Chem. Int. Ed. 2017, 56, 15039-15043; (c) Badir, S. O.; Dumoulin, A.; Matsui, J. K.; Molander, G. A., Synthesis of Reversed C-Acyl Glycosides through Ni/Photoredox Dual Catalysis. Angew. Chem. Int. Ed. 2018, 57, 6610-6613; (d) Dumoulin, A.; Matsui, J. K.; Gutiérrez-Bonet, Á.; Molander, G. A., Synthesis of Non-Classical Arylated C-Saccharides through Nickel/Photoredox Dual Catalysis. Angew. Chem. Int. Ed. 2018, 57, 6614-6618; (e) Matsui, J. K.; Gutiérrez-Bonet, Á.; Rotella, M.; Alam, R.; Gutierrez, O.; Molander, G. A., Photoredox/Nickel-Catalyzed Single-Electron Tsuji-Trost Reaction: Development and Mechanistic Insights. Angew. Chem. Int. Ed. 2018, 57, 15847-15851; (f) Nakajima, K.; Guo, X.; Nishibayashi, Y., Cross-Coupling Reactions of Alkenyl Halides with 4Benzyl-1,4- Dihydropyridines Associated with E to Z Isomerization under Nickel and Photoredox Catalysis. Chem. Asian J. 2018, 13, 3653-3657; (g) Wang, Z.-J.; Zheng, S.; Romero, E.; Matsui, J. K.; Molander, G. A., Regioselective Single-Electron Tsuji-Trost Reaction of Allylic Alcohols: 
A Photoredox/Nickel Dual Catalytic Approach. Org. Lett. 2019, 21, 6543-6547; (h) Luo, Y.; Gutiérrez-Bonet, Á.; Matsui, J. K.; Rotella, M. E.; Dykstra, R.; Gutierrez, O.; Molander, G. A., Oxa- and Azabenzonorbornadienes as Electrophilic Partners under Photoredox/Nickel Dual Catalysis. ACS Catal. 2019, 9, 8835-8842.

9. Wei, Y.; Ben-zvi, B.; Diao, T., Diastereoselective Synthesis of Aryl C-Glycosides from Glycosyl Esters via C-O Bond Homolysis. Angew. Chem. Int. Ed. 2021, 60, 9433-9438.

10. (a) Zhang, P.; Le, C. C.; MacMillan, D. W. C., Silyl Radical Activation of Alkyl Halides in Metallaphotoredox Catalysis: A Unique Pathway for Cross-Electrophile Coupling. J. Am. Chem. Soc. 2016, 138, 8084-8087; (b) Duan, Z.; Li, W.; Lei, A., Nickel-Catalyzed Reductive CrossCoupling of Aryl Bromides with Alkyl Bromides: Et3N as the Terminal Reductant. Org. Lett. 2016, 18, 4012-4015; (c) Paul, A.; Smith, M. D.; Vannucci, A. K., Photoredox-Assisted Reductive Cross-Coupling: Mechanistic Insight into Catalytic Aryl-Alkyl Cross-Couplings. J. Org. Chem. 2017, 82, 1996-2003; (d) Peng, L.; Li, Z.; Yin, G., Photochemical Nickel-Catalyzed Reductive Migratory Cross-Coupling of Alkyl Bromides with Aryl Bromides. Org. Lett. 2018, 20, 18801883; (e) Bacauanu, V.; Cardinal, S.; Yamauchi, M.; Kondo, M.; Fernández, D. F.; Remy, R.; MacMillan, D. W. C., Metallaphotoredox Difluoromethylation of Aryl Bromides. Angew. Chem. Int. Ed. 2018, 57, 12543-12548; (f) Smith, R. T.; Zhang, X.; Rincón, J. A.; Agejas, J.; Mateos, C.; Barberis, M.; García-Cerrada, S.; de Frutos, O.; MacMillan, D. W. C., MetallaphotoredoxCatalyzed Cross-Electrophile Csp3-Csp3 Coupling of Aliphatic Bromides. J. Am. Chem. Soc. 2018, 140, 17433-17438; (g) Zhou, Q.-Q.; Düsel, S. J. S.; Lu, L.-Q.; König, B.; Xiao, W.-J., Alkenylation of unactivated alkyl bromides through visible light photocatalysis. Chem. Commun. 2019, 55, 107-110; (h) Yu, W.; Chen, L.; Tao, J.; Wang, T.; Fu, J., Dual nickel- and photoredoxcatalyzed reductive cross-coupling of aryl vinyl halides and unactivated tertiary alkyl bromides. Chem. Commun. 2019, 55, 5918-5921; (i) Chen, T. Q.; MacMillan, D. W. C., A Metallaphotoredox Strategy for the Cross-Electrophile Coupling of $\alpha$-Chloro Carbonyls with Aryl Halides. Angew. Chem. Int. Ed. 2019, 58, 14584-14588; (j) Sun, S.-Z.; Duan, Y.; Mega, R. S.; Somerville, R. J.; Martin, R., Site-Selective 1,2-Dicarbofunctionalization of Vinyl Boronates through Dual Catalysis. Angew. Chem. Int. Ed. 2020, 59, 4370-4374; (k) Sakai, H. A.; Liu, W.; Le, C. C.; MacMillan, D. W. C., Cross-Electrophile Coupling of Unactivated Alkyl Chlorides. J. Am. Chem. Soc. 2020, 142, 11691-11697; (1) Zhang, R.; Li, G.; Wismer, M.; Vachal, P.; Colletti, S. L.; Shi, Z.-C., Profiling and Application of Photoredox C(sp3)-C(sp2) Cross-Coupling in Medicinal Chemistry. ACS Med. Chem. Lett. 2018, 9, 773-777.

11. (a) Shields, B. J.; Doyle, A. G., Direct C(sp3)-H Cross Coupling Enabled by Catalytic Generation of Chlorine Radicals. J. Am. Chem. Soc. 2016, 138, 12719-12722; (b) Heitz, D. R.; Tellis, J. C.; Molander, G. A., Photochemical Nickel-Catalyzed C-H Arylation: Synthetic Scope and Mechanistic Investigations. J. Am. Chem. Soc. 2016, 138, 12715-12718; (c) Shaw, M. H.; Shurtleff, V. W.; Terrett, J. A.; Cuthbertson, J. D.; MacMillan, D. W. C., Native functionality in triple catalytic cross-coupling: sp\&lt;sup\&gt;3\&lt;/sup\&gt; $\mathrm{C}-\mathrm{H}$ bonds as latent nucleophiles. Science 2016, 352, 1304; (d) Perry, I. B.; Brewer, T. F.; Sarver, P. J.; Schultz, D. M.; DiRocco, D. A.; MacMillan, D. W. C., Direct arylation of strong aliphatic C-H bonds. Nature 2018, 560, 7075; (e) Huang, L.; Rueping, M., Direct Cross-Coupling of Allylic C(sp3)-H Bonds with Aryl- and Vinylbromides by Combined Nickel and Visible-Light Catalysis. Angew. Chem. Int. Ed. 2018, 57, 10333-10337; (f) Zhao, X.; Deng, C.; Meng, D.; Ji, H.; Chen, C.; Song, W.; Zhao, J., NickelCoordinated Carbon Nitride as a Metallaphotoredox Platform for the Cross-Coupling of Aryl Halides with Alcohols. ACS Catal. 2020, 10, 15178-15185; (g) Thullen, S. M.; Treacy, S. M.; 
Rovis, T., Regioselective Alkylative Cross-Coupling of Remote Unactivated C(sp3)-H Bonds. $J$. Am. Chem. Soc. 2019, 141, 14062-14067.

12. Parasram, M.; Shields, B. J.; Ahmad, O.; Knauber, T.; Doyle, A. G., Regioselective CrossElectrophile Coupling of Epoxides and (Hetero)aryl Iodides via Ni/Ti/Photoredox Catalysis. ACS Catal. 2020, 10, 5821-5827.

13. Steiman, T. J.; Liu, J.; Mengiste, A.; Doyle, A. G., Synthesis of $\beta$-Phenethylamines via $\mathrm{Ni} /$ Photoredox Cross-Electrophile Coupling of Aliphatic Aziridines and Aryl Iodides. J. Am. Chem. Soc. 2020, 142, 7598-7605.

14. Dauncey, E. M.; Dighe, S. U.; Douglas, J. J.; Leonori, D., A dual photoredox-nickel strategy for remote functionalization via iminyl radicals: radical ring-opening-arylation, vinylation and -alkylation cascades. Chem. Sci. 2019, 10, 7728-7733.

15. Kammer, L. M.; Badir, S. O.; Hu, R.-M.; Molander, G. A., Photoactive electron donoracceptor complex platform for Ni-mediated C(sp3)-C(sp2) bond formation. Chem. Sci. 2021, 12, 5450-5457.

16. Yi, J.; Badir, S. O.; Kammer, L. M.; Ribagorda, M.; Molander, G. A., Deaminative Reductive Arylation Enabled by Nickel/Photoredox Dual Catalysis. Org. Lett. 2019, 21, 33463351.

17. Knauber, T.; Chandrasekaran, R.; Tucker, J. W.; Chen, J. M.; Reese, M.; Rankic, D. A.; Sach, N.; Helal, C., Ru/Ni Dual Catalytic Desulfinative Photoredox Csp2-Csp3 Cross-Coupling of Alkyl Sulfinate Salts and Aryl Halides. Org. Lett. 2017, 19, 6566-6569.

18. Vara, B. A.; Patel, N. R.; Molander, G. A., O-Benzyl Xanthate Esters under Ni/Photoredox Dual Catalysis: Selective Radical Generation and Csp3-Csp2 Cross-Coupling. ACS Catal. 2017, 7, 3955-3959.

19. (a) Sato, Y.; Nakamura, K.; Sumida, Y.; Hashizume, D.; Hosoya, T.; Ohmiya, H., Generation of Alkyl Radical through Direct Excitation of Boracene-Based Alkylborate. J. Am. Chem. Soc. 2020, 142, 9938-9943; (b) Sato, Y.; Miyamoto, Y.; Sumida, Y.; Hosoya, T.; Ohmiya, H., Boracene-based alkylborate enabled Ni/Ir hybrid catalysis. Org. Biomol. Chem. 2020, 18, 6598-6601.

20. Chen, Y.; Wang, X.; He, X.; An, Q.; Zuo, Z., Photocatalytic Dehydroxymethylative Arylation by Synergistic Cerium and Nickel Catalysis. J. Am. Chem. Soc. 2021, 143, 4896-4902.

21. (a) Hannah E., B.; Takuya, O.; Takahiro, K.; Ryan, S., Concise Synthesis of GB22 by EndoSelective Siloxycyclopropane Arylation. 2019; (b) Huang, L.; Ji, T.; Rueping, M., Remote NickelCatalyzed Cross-Coupling Arylation via Proton-Coupled Electron Transfer-Enabled C-C Bond Cleavage. J. Am. Chem. Soc. 2020, 142, 3532-3539.

22. (a) Kulinkovich, O. G., The Chemistry of Cyclopropanols. Chem. Rev. 2003, 103, 25972632; (b) Nikolaev, A.; Orellana, A., Transition-Metal-Catalyzed C-C and C-X Bond-Forming Reactions Using Cyclopropanols. Synthesis 2016, 48, 1741-1768; (c) McDonald, T. R.; Mills, L. R.; West, M. S.; Rousseaux, S. A. L., Selective Carbon-Carbon Bond Cleavage of Cyclopropanols. Chem. Rev. 2021, 121, 3-79.

23. Woźniak, Ł.; Magagnano, G.; Melchiorre, P., Enantioselective Photochemical Organocascade Catalysis. Angew. Chem. Int. Ed. 2018, 57, 1068-1072.

24. (a) Laktsevich-Iskryk, M. V.; Varabyeva, N. A.; Kazlova, V. V.; Zhabinskii, V. N.; Khripach, V. A.; Hurski, A. L., Visible-Light-Promoted Catalytic Ring-Opening Isomerization of 1,2-Disubstituted Cyclopropanols to Linear Ketones. Eur. J. Org. Chem. 2020, 2020, 2431-2434;

(b) Laktsevich-Iskryk, M. V.; Krech, A. V.; Zhabinskii, V. N.; Khripach, V. A.; Hurski, A. L., 
Photocatalytic Stoichiometric Oxidant-Free Synthesis of Linear Unsaturated Ketones from 1,2Disubstituted Cyclopropanols. Synthesis 2021, 53, 1077-1086.

25. Shang, T.-Y.; Lu, L.-H.; Cao, Z.; Liu, Y.; He, W.-M.; Yu, B., Recent advances of 1,2,3,5tetrakis(carbazol-9-yl)-4,6-dicyanobenzene (4CzIPN) in photocatalytic transformations. Chem. Commun. 2019, 55, 5408-5419.

26. Nithiy, N.; Rosa, D.; Orellana, A., Carbon-Carbon Bond Formation through Palladium Homoenolates. Synthesis 2013, 45, 3199-3210.

27. For the reactions proceeding through formation of RO-NiII species, see: (a) Terrett, J. A.; Cuthbertson, J. D.; Shurtleff, V. W.; MacMillan, D. W. C., Switching on elusive organometallic mechanisms with photoredox catalysis. Nature 2015, 524, 330-334; (b) Zhu, B.; Yan, L.-K.; Geng, Y.; Ren, H.; Guan, W.; Su, Z.-M., IrIII/NiII-Metallaphotoredox catalysis: the oxidation state modulation mechanism versus the radical mechanism. Chem. Commun. 2018, 54, 5968-5971; (c) Yang, L.; Huang, Z.; Li, G.; Zhang, W.; Cao, R.; Wang, C.; Xiao, J.; Xue, D., Synthesis of Phenols: Organophotoredox/Nickel Dual Catalytic Hydroxylation of Aryl Halides with Water. Angew. Chem. Int. Ed. 2018, 57, 1968-1972; (d) Cavedon, C.; Madani, A.; Seeberger, P. H.; Pieber, B., Semiheterogeneous Dual Nickel/Photocatalytic (Thio)etherification Using Carbon Nitrides. Org. Lett. 2019, 21, 5331-5334; (e) Yang, L.; Lu, H.-H.; Lai, C.-H.; Li, G.; Zhang, W.; Cao, R.; Liu, F.; Wang, C.; Xiao, J.; Xue, D., Light-Promoted Nickel Catalysis: Etherification of Aryl Electrophiles with Alcohols Catalyzed by a NiII-Aryl Complex. Angew. Chem. Int. Ed. 2020, 59, 12714-12719; see also Ref. ${ }^{2 c, 11 f}$

28. For reviews on alkoxy radicals generation, see: (a) Tsui, E.; Wang, H.; Knowles, R. R., Catalytic generation of alkoxy radicals from unfunctionalized alcohols. Chem. Sci. 2020, 11, 11124-11141; (b) Wu, X.; Zhu, C., Recent advances in alkoxy radical-promoted C-C and C-H bond functionalization starting from free alcohols. Chem. Commun. 2019, 55, 9747-9756.

29. For examples of reactions proceeding through the homolytic dissociation of the Hal-Ni ${ }^{\mathrm{III}}$ bond in the photoexcited complexes, see: (a) Hwang, S. J.; Powers, D. C.; Maher, A. G.; Anderson, B. L.; Hadt, R. G.; Zheng, S.-L.; Chen, Y.-S.; Nocera, D. G., Trap-Free Halogen Photoelimination from Mononuclear Ni(III) Complexes. J. Am. Chem. Soc. 2015, 137, 6472-6475; (b) Kariofillis, S. K.; Doyle, A. G., Synthetic and Mechanistic Implications of Chlorine Photoelimination in Nickel/Photoredox C(sp3)-H Cross-Coupling. Acc. Chem. Res. 2021, 54, 988-1000; see also Ref. $11 \mathrm{~b}$

30. For an example of the reaction proceeding through the homolytic Ni-C bond cleavage, see:Ting, S. I.; Garakyaraghi, S.; Taliaferro, C. M.; Shields, B. J.; Scholes, G. D.; Castellano, F. N.; Doyle, A. G., 3d-d Excited States of Ni(II) Complexes Relevant to Photoredox Catalysis: Spectroscopic Identification and Mechanistic Implications. J. Am. Chem. Soc. 2020, 142, 58005810. 


\section{Supporting Information}

$\mathrm{Ti}(\mathrm{OiPr})_{4}$-enabled dual photoredox and nickel-catalyzed arylation and alkenylation of cyclopropanols

Nastassia Varabyeva, ${ }^{a, \dagger}$ Maryia Barysevich, ${ }^{a, \dagger}$ Yauhen Aniskevich ${ }^{b}$ and Alaksiej Hurski $^{a *}$

anstitute of Bioorganic Chemistry, National Academy of Sciences of Belarus, Kupreviča Str. 5/2, Minsk 220141, Belarus; ${ }^{b}$ Belarusian State University, Niezaliežnasci Av. 4, Minsk 220030, Belarus

Emal: AHurski@iboch.by

\section{Table of Contents}

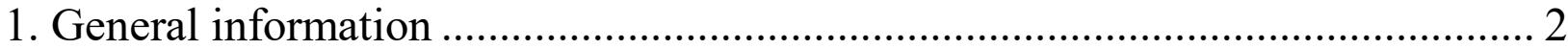

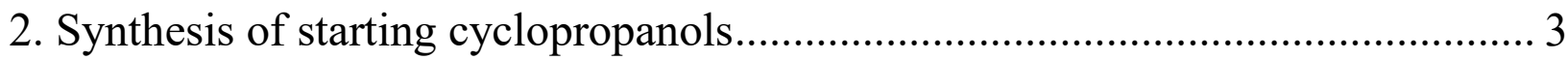

3. Selected optimizations studies on $\mathrm{Ni} /$ photoredox catalyzed alkenylation.............9

4. Arylation and alkenylation of cyclopropanols ..................................................... 11

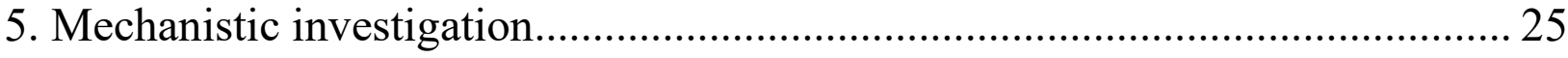

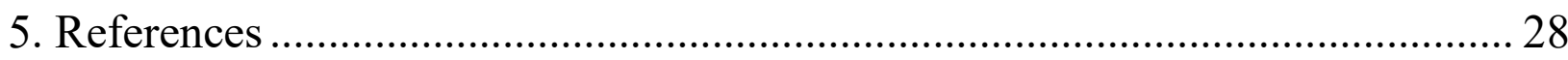

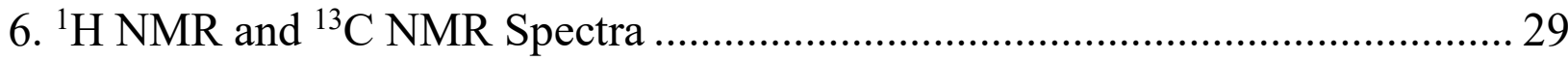




\section{General information}

All solvents were purchased from commercial sources and, unless otherwise noted, used without further purification. If necessary, solvents were distilled and dried before use by standard methods. Flash column chromatography was performed through silica gel (200-300 mesh). Thin layer chromatography (TLC) was performed using Silica gel 60 F254 plates and visualized using UV light or phosphoromolybdic acid. Photochemical reactions were carried with two $20 \mathrm{~W}$ blue light $(400 \mathrm{~nm})$ projectors or with three $10 \mathrm{~W}$ purple $(395 \mathrm{~nm})$ light projectors. The organic dye 2,4,5,6-Tetra(9H-carbazol-9-yl)isophthalonitrile (4CzIPN) and $\mathrm{NiCl}_{2} \cdot$ bpy catalyst were synthesized using reported procedures ${ }^{1,2}$. NMR spectra were obtained on a Bruker AVANCE 500 spectrometer and calibrated using residual solvent as an internal reference $\left[{ }^{1} \mathrm{H}\right.$ NMR: $\left.\mathrm{CDCl}_{3}(7.26) ;{ }^{13} \mathrm{C} \mathrm{NMR}: \mathrm{CDCl}_{3}(77.16)\right]$. Chemical shifts $(\delta)$ are given in ppm and coupling constants $(\mathrm{J})$ are given in Hertz (Hz). Multiplicity was indicated as follows: s (singlet), d (doublet), t (triplet), $q$ (quartet), hept (heptet) $\mathrm{m}$ (multiplet), bs (broad singlet), or combinations thereof. High resolution MS were obtained on Agilent technologies 6550 iFunnel Q-TOF LC/MS system using electrospray ionization (ESI) method. Optical rotations were measured using an Autopol III automatic polarimeter (2-mL cell, $1 \mathrm{dm}$ path length; concentration (c) is in $\mathrm{g} / 100 \mathrm{~mL}$ and $[\alpha]_{\mathrm{D}}$ values are in degrees.). Gas chromatography (GC) was performed on an Agilent $8860 \mathrm{GC}$ instrument using Astec CHIRALDEX B-DM $(30 \mathrm{~m} \times 0.25 \mathrm{~mm})$ column. Cyclic voltammetry was performed in a conventional three-electrode one compartment closed cell with $\mathrm{Pt}$ wire working electrode, Pt sheet counter electrode, and Ag wire pseudoreference electrode; potential of $\mathrm{Ag}$ pseudoreference electrode was calibrated by ferrocenium/ferrocene redox couple (the measurements were performed under dry Ar atmosphere, the electrolyte solution was $0.1 \mathrm{M} \mathrm{NBu}_{4} \mathrm{OTf}$ in acetonitrile). 


\section{Synthesis of starting cyclopropanols}

\section{General procedure A:}

To a stirred solution of ester (1 eq.) and $\mathrm{Ti}(\mathrm{O} i \mathrm{Pr})_{4}(1$ eq. $)$ in dry $\mathrm{THF}$ or $\mathrm{Et}_{2} \mathrm{O}(0.5 \mathrm{M})$ a solution of a Gringnard reagent in dry THF or $\mathrm{Et}_{2} \mathrm{O}$ (1-1.4 M, 4 eq.) was added over $0.5 \mathrm{~h}$ at room temperature under argon atmosphere. The reaction was stirred for $30 \mathrm{~min}$ and quenched with saturated aqueous $\mathrm{NH}_{4} \mathrm{Cl}(0.14 \mathrm{~mL}$ per $1 \mathrm{mmol}$ of Grignard reagent) at $0{ }^{\circ} \mathrm{C}$. The suspension was stirred for $15 \mathrm{~min}$, filtered through Celite $^{\circledR}$ and the precipitate was washed with EtOAc. The filtrate was concentrated under reduced pressure and the residue was purified by flash column chromatography on silica gel using PE and EtOAc as the eluent.

\section{General procedure B:}

To a stirred solution of ester ( 1 eq. $)$, alkene ( 1 eq.) and $\mathrm{Ti}(\mathrm{O} i \mathrm{Pr})_{4}(1$ eq. $)$ in dry THF or $\mathrm{Et}_{2} \mathrm{O}(0.5 \mathrm{M})$ a solution of a Gringnard reagent in dry THF or $\mathrm{Et}_{2} \mathrm{O}$ (1-1.4 M, 4 eq.) was added over $0.5 \mathrm{~h}$ at room temperature under argon atmosphere. The reaction was stirred for $30 \mathrm{~min}$ and quenched with saturated aqueous $\mathrm{NH}_{4} \mathrm{Cl}(0.14 \mathrm{~mL}$ per 1 mmol of Grignard reagent) at $0{ }^{\circ} \mathrm{C}$. The suspension was stirred for $15 \mathrm{~min}$, filtered through Celite ${ }^{\circledR}$ and the precipitate was washed with EtOAc. The filtrate was concentrated under reduced pressure and the residue was purified by flash column chromatography on silica gel using PE and EtOAc as the eluent.

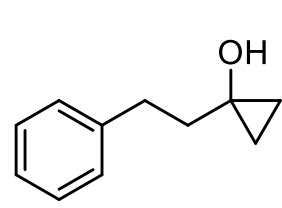

$11 \mathrm{a}$

1-(2-phenylethyl)cyclopropan-1-ol (11a): Synthesized according to the general procedure A from methyl 3-phenylpropionate and ethylmagnesium bromide in THF. The crude product was purified by flash column chromatography on silica gel $(5 \rightarrow 10 \%$ EtOAc/PE) to provide a colorless oil (633 mg, $3.91 \mathrm{mmol}, 64 \%$ yield); ${ }^{1} \mathbf{H}$ NMR $\left(500 \mathrm{MHz}, \mathrm{CDCl}_{3}\right) \delta 7.32-7.27(\mathrm{~m}, 2 \mathrm{H}), 7.25-7.17(\mathrm{~m}, 3 \mathrm{H}), 2.90-2.83(\mathrm{~m}, 2 \mathrm{H})$, $1.92-1.85(\mathrm{~m}, 2 \mathrm{H}), 1.85$ (br. s, $1 \mathrm{H}), 0.82-0.70(\mathrm{~m}, 2 \mathrm{H}), 0.53-0.41(\mathrm{~m}, 2 \mathrm{H}) .{ }^{13} \mathrm{C}$ NMR $\left(126 \mathrm{MHz}, \mathrm{CDCl}_{3}\right) \delta 142.3,128.6$ (2C), 128.6 (2C), 126.0, 56.0, 40.6, 32.6, $13.8(2 \mathrm{C})$. Data are consistent with those reported in the literature. ${ }^{3}$

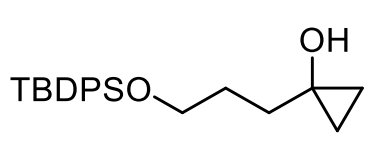

11b

1-(3-(tert-butyldiphenylsilyl)oxy)propyl)cyclopropan-1-ol (11b): Synthesized according to the general procedure A from isopropyl 4-((tert-butyldiphenylsilyl)oxy)butanoate and ethylmagnesium bromide in THF. The crude product was

purified by flash column chromatography on silica gel (10\% EtOAc/PE) to provide a colorless oil (804 mg, $2.27 \mathrm{mmol}, 87 \%$ yield); ${ }^{1} \mathbf{H}$ NMR (500 MHz, $\left.\mathrm{CDCl}_{3}\right) \delta 7.71$ -7.67 (m, 4H), $7.47-7.37$ (m, 6H), 3.76 (t, $J=5.7 \mathrm{~Hz}, 2 \mathrm{H}), 3.26$ (br. s, $1 \mathrm{H}), 1.81$ 
$-1.75(\mathrm{~m}, 2 \mathrm{H}), 1.69(\mathrm{t}, J=6.8 \mathrm{~Hz}, 2 \mathrm{H}), 1.05(\mathrm{~s}, 9 \mathrm{H}), 0.80-0.75(\mathrm{~m}, 2 \mathrm{H}), 0.47-$ $0.43(\mathrm{~m}, 2 \mathrm{H}) .{ }^{13} \mathrm{C}$ NMR $\left(126 \mathrm{MHz}, \mathrm{CDCl}_{3}\right) \delta 135.7$ (4C), 133.6 (2C), $129.9(2 \mathrm{C})$, 127.9 (4C), 64.6, 55.6, 36.1 , 31.2, 29.6, 26.9 (3C), 19.3, 13.7. HRMS (ESI+): $m / z$ calc'd for $\mathrm{C}_{22} \mathrm{H}_{31} \mathrm{O}_{2} \mathrm{Si}[\mathrm{M}+\mathrm{H}]^{+}:$355.2088, found 355.2084.

\section{1-(4-methylpent-3-en-1-yl)cyclopropane-1-ol (11c): Synthesized}

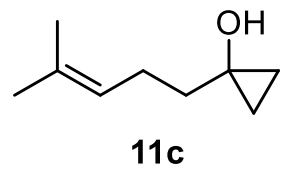

according to the general procedure A from ethyl 5-methylhex-4enoate and ethylmagnesium bromide in THF. The crude product was purified by flash column chromatography on silica gel $(15 \%$ EtOAc/PE) to provide a colorless oil $(605 \mathrm{mg}, 4.31 \mathrm{mmol}, 67 \%$ yield $) ;{ }^{1} \mathbf{H}$ NMR $\left(500 \mathrm{MHz}, \mathrm{CDCl}_{3}\right) \delta 4.79(\mathrm{t}, J=5.9 \mathrm{~Hz}, 1 \mathrm{H}), 3.72(\mathrm{dq}, J=9.4,7.0 \mathrm{~Hz}, 2 \mathrm{H}), 3.59(\mathrm{~s}$, $1 \mathrm{H}), 3.56(\mathrm{dq}, J=9.5,7.1 \mathrm{~Hz}, 2 \mathrm{H}), 1.89(\mathrm{~d}, J=5.8 \mathrm{~Hz}, 2 \mathrm{H}), 1.23(\mathrm{t}, J=7.1 \mathrm{~Hz}, 6 \mathrm{H})$, $0.81-0.70(\mathrm{~m}, 2 \mathrm{H}), 0.49-0.39(\mathrm{~m}, 2 \mathrm{H}) .{ }^{13} \mathbf{C ~ N M R}\left(126 \mathrm{MHz}, \mathrm{CDCl}_{3}\right) \delta 103.1$, $61.9,53.3,41.1,15.5,12.6$.

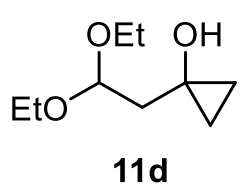

\section{1-(2,2-diethoxyethyl)cyclopropane-1-ol}

(11d): Synthesized according to the general procedure A from ethyl 3,3diethoxypropanoate and ethylmagnesium bromide in THF. The crude product was purified by flash column chromatography on silica gel $(10 \rightarrow 20 \%$ EtOAc/PE) to provide a colorless oil $(357 \mathrm{mg}, 2.05 \mathrm{mmol}, 39 \%$ yield); ${ }^{1} \mathbf{H}$ NMR $\left(500 \mathrm{MHz}, \mathrm{CDCl}_{3}\right) \delta 4.79(\mathrm{t}, J=5.9 \mathrm{~Hz}, 1 \mathrm{H}), 3.72(\mathrm{dq}, J=9.5,7.1 \mathrm{~Hz}$, 2H), $3.59(\mathrm{~s}, 1 \mathrm{H}), 3.56(\mathrm{dq}, J=9.5,7.1 \mathrm{~Hz}, 2 \mathrm{H}), 1.89(\mathrm{~d}, J=5.9 \mathrm{~Hz}, 2 \mathrm{H}), 1.23(\mathrm{t}, J$ $=7.1 \mathrm{~Hz}, 6 \mathrm{H}), 0.81-0.70(\mathrm{~m}, 2 \mathrm{H}), 0.49-0.39(\mathrm{~m}, 2 \mathrm{H}) .{ }^{13} \mathbf{C} \mathbf{~ N M R}(126 \mathrm{MHz}$, $\left.\mathrm{CDCl}_{3}\right) \delta 103.1,61.9,53.3,41.1,15.5,12.6$. Data are consistent with those reported in the literature. ${ }^{4}$

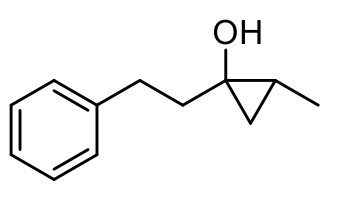

$11 \mathrm{e}$

2-methyl-1-phenethylcyclopropan-1-ol (11e): Synthesized according to the general procedure A from methyl 3phenylpropionate and propylmagnesium bromide in THF. The crude product was purified by flash column chromatography on silica gel $(5 \rightarrow 10 \%$ EtOAc/PE) to provide a colorless oil $(157 \mathrm{mg}, 0.89 \mathrm{mmol}, 80 \%$ yield); ${ }^{1} \mathbf{H}$ NMR $\left(500 \mathrm{MHz}, \mathrm{CDCl}_{3}\right) \delta 7.33-7.27(\mathrm{~m}, 2 \mathrm{H}), 7.26-7.18(\mathrm{~m}, 3 \mathrm{H}), 2.92$ $-2.85(\mathrm{~m}, 2 \mathrm{H}), 1.93-1.85(\mathrm{~m}, 2 \mathrm{H}), 1.80(\mathrm{~s}, 1 \mathrm{H}), 1.13-1.05(\mathrm{~m}, 1 \mathrm{H}), 1.02(\mathrm{~d}, J=$ $6.1 \mathrm{~Hz}, 3 \mathrm{H}), 0.86(\mathrm{dd}, J=9.7,5.3 \mathrm{~Hz}, 1 \mathrm{H}), 0.10-0.04(\mathrm{~m}, 1 \mathrm{H}) .{ }^{13} \mathbf{C}$ NMR $(126$ $\left.\mathrm{MHz}, \mathrm{CDCl}_{3}\right) \delta 142.6,128.6,128.6,126.0,59.0,36.4,32.7,20.9,20.1,14.3$. Data are consistent with those reported in the literature. ${ }^{3}$

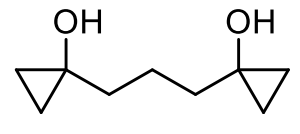

$11 f$

1,1'-(propane-1,3-diyl)bis(cyclopropan-1-ol) (11f): Synthesized according to the general procedure A from dimethyl glutarate and 
ethylmagnesium bromide ( 8 equiv.) in THF. The crude product was purified by flash column chromatography on silica gel $(30 \% \mathrm{EtOAc} / \mathrm{PE})$ to provide white crystals (402 mg, $2.57 \mathrm{mmol}, 42 \%$ yield); ${ }^{1} \mathrm{H}$ NMR (500 MHz, $\left.\mathrm{CDCl}_{3}\right) \delta 2.05$ (s, 2H), 1.80 $-1.71(\mathrm{~m}, 2 \mathrm{H}), 1.66-1.56(\mathrm{~m}, 4 \mathrm{H}), 0.80-0.70(\mathrm{~m}, 4 \mathrm{H}), 0.51-0.41(\mathrm{~m}, 4 \mathrm{H}) .{ }^{13} \mathrm{C}$ NMR $\left(126 \mathrm{MHz}, \mathrm{CDCl}_{3}\right) \delta 55.9,38.3,22.5,13.7$. Data are consistent with those reported in the literature. ${ }^{5}$

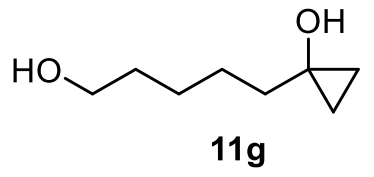

1-(5-hydroxypentyl)cyclopropan-1-ol (11g): Synthesized according to the general procedure A from caprolactone and ethylmagnesium bromide in THF. The crude product was purified by flash column chromatography on silica gel $(10 \rightarrow 15 \%$ EtOAc/PE) to provide a colorless oil $(1.04 \mathrm{~g}, 7.19 \mathrm{mmol}, 55 \%$ yield $) ;{ }^{1} \mathrm{H} \mathrm{NMR}\left(500 \mathrm{MHz}, \mathrm{CDCl}_{3}\right)$ $\delta 3.65(\mathrm{t}, J=6.6 \mathrm{~Hz}, 2 \mathrm{H}), 1.65-1.49(\mathrm{~m}, 6 \mathrm{H}), 1.48-1.35(\mathrm{~m}, 2 \mathrm{H}), 0.79-0.66(\mathrm{~m}$, $2 \mathrm{H}), 0.51-0.35(\mathrm{~m}, 2 \mathrm{H}) .{ }^{13} \mathrm{C} \mathrm{NMR}\left(126 \mathrm{MHz}, \mathrm{CDCl}_{3}\right) \delta 63.0,55.8,38.4,32.8,25.8$, 13.7. Data are consistent with those reported in the literature. ${ }^{6}$

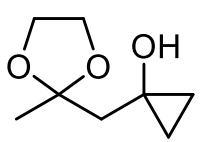

$11 \mathrm{~h}$

\section{1-((2-methyl-1,3-dioxolan-2-yl)methyl)cyclopropane-1-ol (11h):}

Synthesized according to the general procedure A from ethyl 2-(2methyl-1,3-dioxolan-2-yl)acetate and ethylmagnesium bromide in THF.

The crude product was purified by flash column chromatography on silica gel (15\% EtOAc/PE) to provide a colorless oil (180.4 mg, $1.14 \mathrm{mmol}, 40 \%$ yield); ${ }^{1} \mathbf{H}$ NMR (500 MHz, $\left.\mathrm{CDCl}_{3}\right) \delta 4.06-3.97$ (m, 4H), 3.85 (br. s, 1H), 1.94 (s, 2H), $1.44(\mathrm{~s}, 3 \mathrm{H}), 0.78-0.73(\mathrm{~m}, 2 \mathrm{H}), 0.51-0.47(\mathrm{~m}, 2 \mathrm{H}) .{ }^{13} \mathbf{C}$ NMR $(126 \mathrm{MHz}$, $\left.\mathrm{CDCl}_{3}\right) \delta 111.3,64.7(2 \mathrm{C}), 53.3,45.2,24.9,12.7$ (2C). Data are consistent with those reported in the literature. ${ }^{?}$

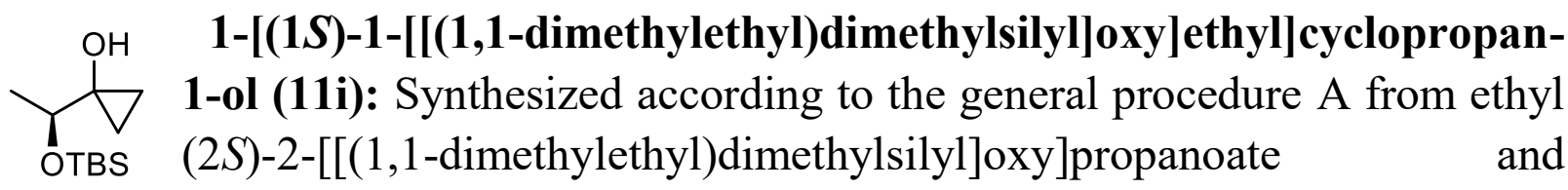

11i ethylmagnesium bromide in THF. The crude product was purified by flash column chromatography on silica gel $(5 \% \mathrm{EtOAc} / \mathrm{PE})$ to provide a colorless oil (275 mg, $1.27 \mathrm{mmol}, 60 \%$ yield $) ;[\boldsymbol{\alpha}]_{\mathbf{D}}{ }^{\mathbf{2 0}}=+4.58\left(\mathrm{c}=0.50\right.$ in $\left.\mathrm{CHCl}_{3}\right) ;{ }^{1} \mathbf{H}$ NMR $\left(500 \mathrm{MHz}, \mathrm{CDCl}_{3}\right) \delta 3.41(\mathrm{q}, \mathrm{J}=6.3 \mathrm{~Hz}, 1 \mathrm{H}), 2.47$ (br. s, $\left.1 \mathrm{H}\right), 1.24(\mathrm{~d}, \mathrm{~J}=6.3$ $\mathrm{Hz}, 3 \mathrm{H}), 0.90(\mathrm{~s}, 9 \mathrm{H}), 0.82-0.78(\mathrm{~m}, 1 \mathrm{H}), 0.76-0.72(\mathrm{~m}, 1 \mathrm{H}), 0.49-0.46(\mathrm{~m}, 2 \mathrm{H})$, $0.07(\mathrm{~s}, 6 \mathrm{H}) .{ }^{13} \mathrm{C}$ NMR $\left(126 \mathrm{MHz}, \mathrm{CDCl}_{3}\right) \delta$ 73.2, 59.6, 26.0 (3C), 19.9, 18.2, 13.6, $10.0,-4.4,-4.4$. 
TBSO

11j

product was purified by flash column chromatography on silica gel $(5 \%$ EtOAc/PE) to provide a colorless oil $\left(361 \mathrm{mg}, 1.57 \mathrm{mmol}, 73 \%\right.$ yield); $[\boldsymbol{\alpha}]_{\mathbf{D}}{ }^{\mathbf{2 0}}=+3.64(\mathrm{c}=0.53$ in $\left.\mathrm{CHCl}_{3}\right)^{1} \mathbf{H}$ NMR $\left(500 \mathrm{MHz}, \mathrm{CDCl}_{3}\right) \delta 3.91(\mathrm{dd}, \mathrm{J}=9.6,4.0 \mathrm{~Hz}, 1 \mathrm{H}), 3.68(\mathrm{dd}, \mathrm{J}$ $=9.6,5.0 \mathrm{~Hz}, 1 \mathrm{H}), 3.60$ (br. s, $1 \mathrm{H}), 1.41-1.34(\mathrm{~m}, 1 \mathrm{H}), 1.02(\mathrm{~d}, \mathrm{~J}=7.1 \mathrm{~Hz}, 3 \mathrm{H})$, 0.91 (s, 9H), 0.75 (ddd, J = 10.9, 5.8, $4.8 \mathrm{~Hz}, 1 \mathrm{H}$ ), 0.67 (ddd, J = 10.8, 5.8, 5.3 Hz, 1H), 0.47 (ddd, J = 10.8, 5.9, $4.8 \mathrm{~Hz}, 1 \mathrm{H}$ ), 0.37 (ddd, $\mathrm{J}=10.9,5.9,5.3 \mathrm{~Hz}, 1 \mathrm{H}$ ), 0.09 (s, 6H). ${ }^{13}$ C NMR $\left(126 \mathrm{MHz}, \mathrm{CDCl}_{3}\right) \delta 68.5,59.7,40.8,26.0$ (3C), 18.3, 13.3, 13.0, $11.4,-5.4,-5.5$. Data are consistent with those reported in the literature. ${ }^{8}$

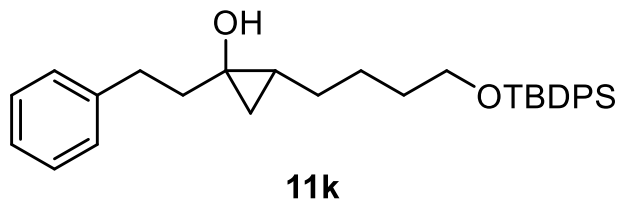

$11 \mathrm{k}$

2-(4-((tert-butyldiphenylsilyl)oxy)butyl)-1phenethylcyclopropan-1-ol (11k): Synthesized according to the general procedure B from ethyl 3phenylpropionate,

tert-butyl(hex-5-en-1yloxy)diphenylsilane and cyclopentylmagnesium chloride in THF. The crude product was purified by flash column chromatography on silica gel (10\% EtOAc/PE) to provide a colorless oil $(730 \mathrm{mg}, 1.54 \mathrm{mmol}, 51 \%$ yield $) ;{ }^{1} \mathrm{H}$ NMR $(500 \mathrm{MHz}$, $\left.\mathrm{CDCl}_{3}\right) \delta 7.72-7.61(\mathrm{~m}, 4 \mathrm{H}), 7.45-7.33(\mathrm{~m}, 6 \mathrm{H}), 7.32-7.27(\mathrm{~m}, 2 \mathrm{H}), 7.24-7.17$ $(\mathrm{m}, 3 \mathrm{H}), 3.66(\mathrm{t}, J=6.4 \mathrm{~Hz}, 2 \mathrm{H}), 2.93-2.80(\mathrm{~m}, 2 \mathrm{H}), 2.00-1.91(\mathrm{~m}, 1 \mathrm{H}), 1.82-$ $1.73(\mathrm{~m}, 2 \mathrm{H}), 1.67-1.38(\mathrm{~m}, 6 \mathrm{H}), 1.05(\mathrm{~s}, 9 \mathrm{H}), 0.94-0.79(\mathrm{~m}, 2 \mathrm{H}), 0.11-0.05(\mathrm{~m}$, $1 \mathrm{H}) .{ }^{13} \mathrm{C}$ NMR $\left(126 \mathrm{MHz}, \mathrm{CDCl}_{3}\right) \delta 142.6,135.7,134.3,129.7,128.6,128.6,127.7$, 126.0, 64.0, 59.0, 36.6, 32.7, 32.5, 29.4, 27.0, 26.3, 26.0, 19.8, 19.4. HRMS (ESI+): $m / z$ calc'd for $\mathrm{C}_{31} \mathrm{H}_{40} \mathrm{NaO}_{2} \mathrm{Si}[\mathrm{M}+\mathrm{Na}]^{+}:$495.2690, found 495.2686.

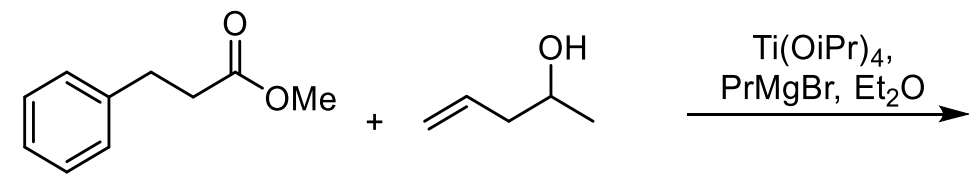<smiles>CCC(O)C[C@@H]1C[C@@]1(O)CCc1ccccc1</smiles>

$( \pm)-\mathbf{S 1}$
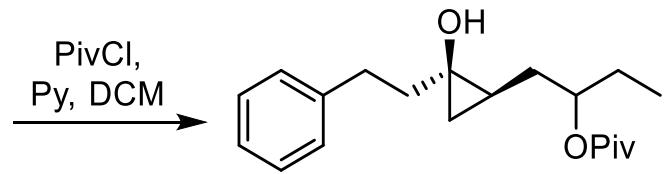

$( \pm)-111$

\section{1-(2-hydroxy-2-phenethylcyclopropyl)butan-2-yl pivalate (( \pm )-11l):}

To a stirred solution of methyl 3-phenylpropionate (492 mg, 3 mmol, 1 eq.), pent-4en-2-ol (300 mg, 3 mmol, 1 eq.) and $\mathrm{Ti}(\mathrm{O} i \mathrm{Pr})_{4}(0.89 \mathrm{~mL}, 3 \mathrm{mmol}, 1$ eq. $)$ in dry $\mathrm{Et}_{2} \mathrm{O}$ $(25 \mathrm{~mL})$ a solution of $\mathrm{PrMgBr}$ in $\mathrm{Et}_{2} \mathrm{O}(22 \mathrm{ml}, 24 \mathrm{mmol}, 1.1 \mathrm{M}, 8$ eq. $)$ was added 
over $1 \mathrm{~h}$ at room temperature under argon atmosphere. The reaction was stirred for $30 \mathrm{~min}$ and quenched with saturated aqueous $\mathrm{NH}_{4} \mathrm{Cl}$ solution $(3.5 \mathrm{~mL})$ at $0{ }^{\circ} \mathrm{C}$. The suspension was stirred for $15 \mathrm{~min}$, filtered through Celite ${ }^{\circledR}$ and the precipitate was washed with EtOAc $(5 \times 5 \mathrm{~mL})$. The filtrate was concentrated under reduced pressure and the residue was purified by flash column chromatography on silica gel $(10 \rightarrow 15 \%$ EtOAc/PE) to give cyclopropanol $( \pm)-\mathbf{S 1}$ as a colorless oil (313 mg, 1.33 mmol, $45 \%$ yield). ${ }^{1} \mathbf{H}$ NMR $\left(500 \mathrm{MHz}, \mathrm{CDCl}_{3}\right) \delta 7.31-7.25(\mathrm{~m}, 2 \mathrm{H}), 7.23-7.15$ (m, 3H), $3.71-3.66(\mathrm{~m}, 1 \mathrm{H}), 2.91-2.82(\mathrm{~m}, 2 \mathrm{H}), 1.96(\mathrm{dt}, J=14.9,4.5 \mathrm{~Hz}, 1 \mathrm{H})$, $1.91-1.83(\mathrm{~m}, 1 \mathrm{H}), 1.83-1.75(\mathrm{~m}, 1 \mathrm{H}), 1.65-1.53(\mathrm{~m}, 3 \mathrm{H}), 0.95$ (t, $J=7.4 \mathrm{~Hz}$, $3 \mathrm{H}), 0.80-0.71(\mathrm{~m}, 1 \mathrm{H}), 0.66(\mathrm{dd}, J=9.3,5.1 \mathrm{~Hz}, 1 \mathrm{H}), 0.46-0.41(\mathrm{~m}, 1 \mathrm{H}) .{ }^{13} \mathrm{C}$ NMR $\left(126 \mathrm{MHz}, \mathrm{CDCl}_{3}\right) \delta 142.7,128.5$ (2C), 128.5 (2C), 125.8, 72.9, 57.4, 42.0, $34.2,32.4,28.8,19.9,19.5,10.5$.

To a solution of cyclopropanol $( \pm)-\mathbf{S 1}(587 \mathrm{mg}, 2.5 \mathrm{mmol})$ and pyridine $(0.44 \mathrm{ml}$, $5.5 \mathrm{mmol}, 2.2$ eq.) in DCM (9 ml) pivaloyl chloride $(0.61 \mathrm{~mL}, 5 \mathrm{mmol}, 2$ eq. $)$ was added at $0{ }^{\circ} \mathrm{C}$. The reaction mixture was stirred overnight at room temperature, then quenched with saturated aqueous $\mathrm{NaHCO}_{3}$ solution $(5 \mathrm{~mL})$ and extracted with EtOAc $(3 \times 5 \mathrm{~mL})$. The combined organic layers were dried over $\mathrm{Na}_{2} \mathrm{SO}_{4}$ and concentrated under reduced pressure. The residue was purified by column chromatography on silica gel $(10 \%$ EtOAc/PE) to provide cyclopropanol $( \pm)-\mathbf{1 1 1}$ as a colorless oil $(557 \mathrm{mg}, 70 \%) .{ }^{1} \mathbf{H}$ NMR $\left(500 \mathrm{MHz}, \mathrm{CDCl}_{3}\right) \delta 7.30-7.25(\mathrm{~m}, 2 \mathrm{H})$, $7.22-7.15(\mathrm{~m}, 3 \mathrm{H}), 4.96-4.87(\mathrm{~m}, 1 \mathrm{H}), 2.89-2.77(\mathrm{~m}, 2 \mathrm{H}), 2.14(\mathrm{br} \mathrm{s}, 1 \mathrm{H}), 1.90$ $-1.81(\mathrm{~m}, 1 \mathrm{H}), 1.80-1.54(\mathrm{~m}, 5 \mathrm{H}), 1.21(\mathrm{~s}, 9 \mathrm{H}), 0.89(\mathrm{t}, J=7.5 \mathrm{~Hz}, 3 \mathrm{H}), 0.65-$ $0.58(\mathrm{~m}, 2 \mathrm{H}), 0.46-0.37(\mathrm{~m}, 1 \mathrm{H}) .{ }^{13} \mathbf{C}$ NMR $\left(126 \mathrm{MHz}, \mathrm{CDCl}_{3}\right) \delta 178.5,142.4$, 128.5 (4C), 125.9, 75.5, 58.2, 41.6, 39.0, 32.5, 32.4, 27.4, 27.2, 20.7, 19.1, 9.7. Data are consistent with those reported in the literature. ${ }^{9}$
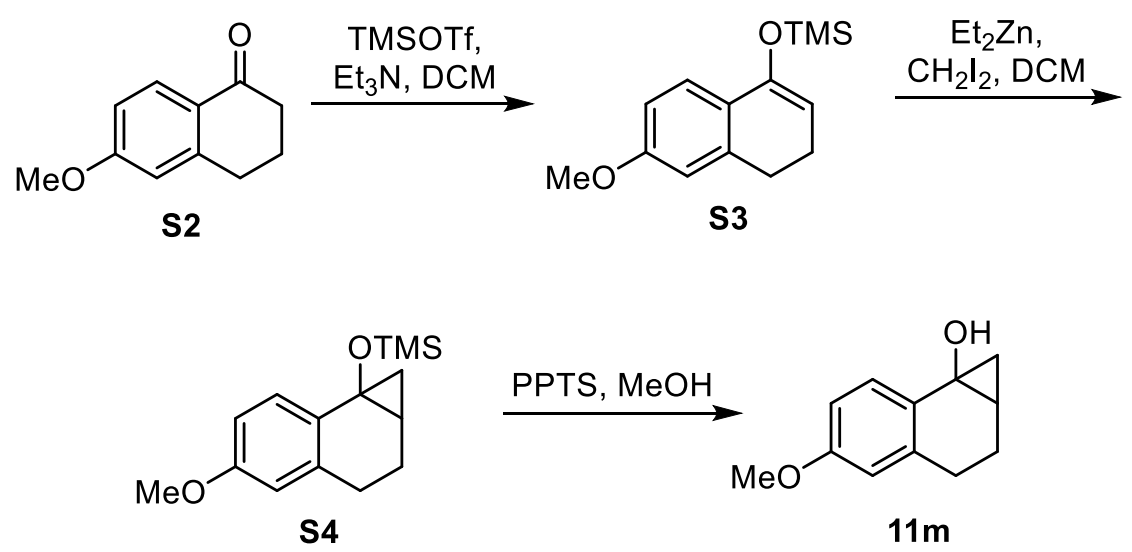

\section{5-methoxy-1,1a,2,3-tetrahydro-7bH-cyclopropa[a]naphthalen-7b-ol (11m):}

To an ice-cold solution of ketone $\mathbf{S 2}\left(500 \mathrm{mg}, 2.8 \mathrm{mmol}, 1\right.$ eq.) and $\mathrm{Et}_{3} \mathrm{~N}$ (1.6 mL, $11.4 \mathrm{mmol}, 4$ eq.) in dry DCM (7 mL) TMSOTf $(1.5 \mathrm{~mL}, 8.5 \mathrm{mmol}, 3$ eq. $)$ was added 
dropwise. After stirring for $1 \mathrm{~h}$ at $0^{\circ} \mathrm{C}$, the reaction mixture was diluted with saturated aqueous $\mathrm{NaHCO}_{3}$ solution $(5 \mathrm{~mL})$ and water $(5 \mathrm{~mL})$. The organic layer was separated and the aqueous layer was extracted with DCM $(2 \times 5 \mathrm{~mL})$. The combined organic layers were dried over $\mathrm{Na}_{2} \mathrm{SO}_{4}$ and concentrated under reduced pressure to give TMS enol ether $\mathbf{S 3}$ as an oil which was used in the next step without further purification.

To an ice-cold solution of the above TMS enol ether $\mathbf{S 3}$ in dry DCM (10 mL) solution of $\mathrm{Et}_{2} \mathrm{Zn}$ in toluene $(9.3 \mathrm{~mL}, 6.5 \mathrm{mmol}, 0.7 \mathrm{M}, 2.3 \mathrm{eq}$.) was added under argon atmosphere followed by $\mathrm{CH}_{2} \mathrm{I}_{2}(0.68 \mathrm{~mL}, 8.4 \mathrm{mmol}, 3$ eq.). The reaction mixture was stirred overnight at room temperature, then quenched with saturated aqueous $\mathrm{NH}_{4} \mathrm{Cl}(5 \mathrm{~mL})$ and extracted with $\mathrm{DCM}(3 \times 5 \mathrm{~mL})$. Combined organic phases were dried over $\mathrm{Na}_{2} \mathrm{SO}_{4}$ and concentrated under reduced pressure to give TMS-protected cyclopropanol $\mathbf{S} \mathbf{4}$ as a colorless oil which was used in the next step without additional purification.

To a solution of the above TMS-protected cyclopropanol $\mathbf{S 4}$ in $\mathrm{MeOH}(2.8 \mathrm{~mL})$ PPTS (704 mg, $2.8 \mathrm{mmol}, 1$ eq.) was added. After stirring for $10 \mathrm{~min}$ at room temperature, pyridine $(0.23 \mathrm{~mL}, 2.8 \mathrm{mmol}, 1$ eq. $)$ was added, and the mixture was evaporated under reduced pressure. The residue was then purified by column chromatography on silica gel $(15 \rightarrow 25 \%$ EtOAc/PE) to provide cyclopropanol $11 \mathbf{m}$ as a colorless oil (388 mg, $2.0 \mathrm{mmol}, 73 \%$ yield over three steps). ${ }^{1} \mathbf{H}$ NMR (500 $\left.\mathrm{MHz}, \mathrm{CDCl}_{3}\right) \delta 7.62(\mathrm{~d}, J=8.5 \mathrm{~Hz}, 1 \mathrm{H}), 6.82(\mathrm{ddd}, J=8.5,2.7,0.9 \mathrm{~Hz}, 1 \mathrm{H}), 6.66-$ $6.61(\mathrm{~m}, 1 \mathrm{H}), 3.79(\mathrm{~s}, 3 \mathrm{H}), 2.61-2.54(\mathrm{~m}, 1 \mathrm{H}), 2.42-2.29(\mathrm{~m}, 2 \mathrm{H}), 1.98(\mathrm{ddt}, J=$ $13.2,6.4,2.2 \mathrm{~Hz}, 1 \mathrm{H}), 1.77$ (tdd, $J=13.2,5.5,3.2 \mathrm{~Hz}, 1 \mathrm{H}), 1.73-1.65(\mathrm{~m}, 1 \mathrm{H})$, $1.20(\mathrm{dd}, J=9.9,5.7 \mathrm{~Hz}, 1 \mathrm{H}), 1.02-0.95(\mathrm{~m}, 1 \mathrm{H}) .{ }^{13} \mathbf{C} \mathbf{~ N M R}\left(126 \mathrm{MHz}, \mathrm{CDCl}_{3}\right) \delta$ $157.8,134.5,132.8,125.3,114.1,111.7,55.4,54.6,26.5,24.2,18.8,16.4$. Data are consistent with those reported in the literature. ${ }^{9}$ 


\section{Selected optimizations studies on $\mathrm{Ni} /$ photoredox catalyzed alkenylation}

General procedure: condition screening

A $1.5 \mathrm{~mL}$ vial equipped with a stirring bar was charged with cyclopropanol 11 a (20 mg, 0.12 mmol, 1 eq.), $\mathrm{NiCl}_{2} \cdot$ bpy ( $1.8 \mathrm{mg}, 6.2 \mu \mathrm{mol}, 5 \mathrm{~mol} \%$ ), 4CzIPN (4.9 mg, 6.2 $\mu \mathrm{mol}, 5 \mathrm{~mol} \%), \mathrm{K}_{2} \mathrm{CO}_{3}\left(51.2 \mathrm{mg}, 0.37 \mathrm{mmol}, 3\right.$ eq.) and $\mathrm{Ti}(\mathrm{O} i \mathrm{Pr})_{4}(73 \mu \mathrm{L}, 0.25$ mmol, 2 eq.). The vial was purged with Ar for 5 min followed by addition of 2bromoprop-1-ene $(21 \mu \mathrm{L}, 0.25 \mathrm{mmol}, 2$ eq.) solution in acetone $(1.2 \mathrm{~mL})$. The resulting mixture was set to stir $(1200 \mathrm{rpm})$ and irradiated with two $20 \mathrm{~W}$ blue LED spotlights ( $5 \mathrm{~cm}$ away) for $15 \mathrm{~h}$. Upon reaction complete the crude mixture was poured into $5 \% \mathrm{H}_{2} \mathrm{SO}_{4}$ solution $(2 \mathrm{~mL})$ and extracted with EtOAc $(3 \times 2 \mathrm{~mL})$. The combined organic layers were washed with water $(2 \mathrm{~mL})$, saturated solution of $\mathrm{NaHCO}_{3}(2 \mathrm{~mL})$, dried over $\mathrm{Na}_{2} \mathrm{SO}_{4}$ and concentrated under reduced pressure. Crude NMR with internal standard (dibromomethane) was recorded.
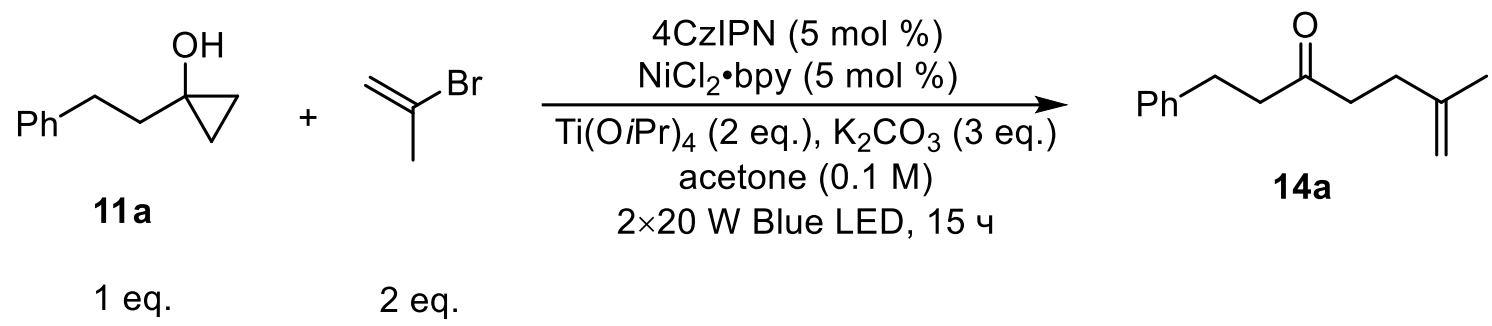

Table S1: Solvent screening

\begin{tabular}{ccc}
\hline $\begin{array}{c}\text { Deviation from standard } \\
\text { conditions }\end{array}$ & Conversion & NMR Yield of 14a \\
\hline none & 62 & 58 \\
MeCN & 77 & 58 \\
THF & 57 & 45 \\
DMA & 53 & 43 \\
acetone $/ i \operatorname{PrOH}(60: 1)$ & 56 & 54 \\
acetone $/ i \operatorname{PrOH}(6: 1)$ & 60 & 60 \\
$i \operatorname{PrOH}$ & - & trace \\
\hline
\end{tabular}



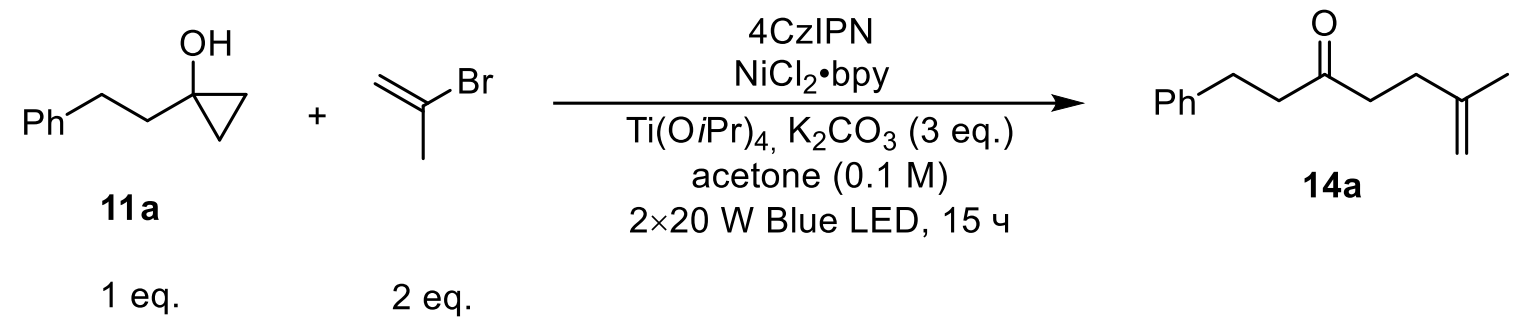

Table S2: Condition evaluation

\begin{tabular}{|c|c|c|}
\hline $\begin{array}{c}\text { Deviation from } \\
\text { standard conditions }\end{array}$ & $\begin{array}{c}\text { Conversion, } \\
\%\end{array}$ & $\begin{array}{l}\text { Yield 14a, } \\
\%^{\mathrm{a}}\end{array}$ \\
\hline $10 \mathrm{~mol} \%$ of $4 \mathrm{CzIPN}$ instead of $5 \mathrm{~mol} \%{ }^{\mathrm{b}}$ & 82 & 59 \\
\hline $10 \mathrm{~mol} \%$ of $\mathrm{NiCl}_{2} \cdot$ bpy instead of $5 \mathrm{~mol} \mathrm{\%}{ }^{\mathrm{c}}$ & 61 & 53 \\
\hline $\begin{array}{c}\mathrm{CPTi}(\mathrm{O} i \operatorname{Pr})_{3} \mathbf{1 5}(1 \text { eq. })^{\mathrm{d}} \\
\text { instead of } \mathbf{1 1 a}(1 \text { eq. })+\mathrm{Ti}(\mathrm{O} i \operatorname{Pr})_{4}(2 \text { eq. })\end{array}$ & 52 & 41 \\
\hline $\begin{array}{l}\left.\mathrm{NiCl}_{2} \cdot \mathrm{DME}(5 \mathrm{~mol} \%)+2,9-\mathrm{Neocuproine}^{\circ}\right) \\
(5 \mathrm{~mol} \%) \text { instead of } \mathrm{NiCl}_{2} \cdot \text { bpy }\end{array}$ & 92 & $82(71)^{\mathrm{e}}$ \\
\hline $\begin{array}{l}\mathrm{NiCl}_{2} \cdot \mathrm{DME}(5 \mathrm{~mol} \%)+6,6 \text {-Dimethyl-2,2'- } \\
\text { bipyridine }(5 \mathrm{~mol} \mathrm{\%}) \text { instead of } \mathrm{NiCl}_{2} \cdot \text { bpy }\end{array}$ & 76 & 45 \\
\hline $\begin{array}{l}\mathrm{NiCl}_{2} \cdot \mathrm{DME}(2.5 \mathrm{~mol} \%)+2,9-\mathrm{Neocuproine} \\
\quad(2.5 \mathrm{~mol} \%) \text { instead of } \mathrm{NiCl}_{2} \cdot \mathrm{bpy}\end{array}$ & nd & nd $(84)^{\mathrm{e}}$ \\
\hline $\operatorname{Ti}(\mathrm{O} t \mathrm{Bu})_{4}$ instead of $\mathrm{Ti}(\mathrm{O} i \mathrm{Pr})_{4}$ & 43 & 33 \\
\hline
\end{tabular}

a Yields were determined by ${ }^{1} \mathrm{H}$ NMR spectroscopy using dibromomethane as an internal standard;

${ }^{\mathrm{b}} \mathrm{MeCN}$ was used as a solvent;

${ }^{\mathrm{c}}$ The reaction was carried out with $5 \%$ of $\mathrm{NiCl}_{2}$.bpy and additional $5 \%$ were added after $7 \mathrm{~h}$;

${ }^{\mathrm{d}} \mathrm{CP}$ - 1-phenethylcyclopropoxy substituent.

e Isolated yields are given in parentheses (the reactions were carried out with $0.25 \mathrm{mmol}$ of starting material); 


\section{Arylation and alkenylation of cyclopropanols}

\section{General procedure A:}

A $4 \mathrm{~mL}$ vial equipped with a stirring bar was charged with cyclopropanol (0.25 mmol, 1 eq.), aryl halide (0.50 mmol, 2 eq.), 4CzIPN (9.9 mg, $12.5 \mu \mathrm{mol}$, $5 \mathrm{~mol} \%), \mathrm{NiCl}_{2} \cdot$ bpy (3.6 mg, $\left.12.5 \mu \mathrm{mol}, 5 \mathrm{~mol} \%\right)$, Ti(O $\left.i \operatorname{Pr}\right)_{4}(148 \mu \mathrm{L}, 0.50 \mathrm{mmol}$, 2 eq.) and $\mathrm{K}_{2} \mathrm{CO}_{3}(103.5 \mathrm{mg}, 0.75 \mathrm{mmol}, 3$ eq.). The vial was purged with Ar for $10 \mathrm{~min}$, followed by addition of acetone $(2.5 \mathrm{~mL})$. The resulting mixture was set to stir (1200 rpm) and irradiated with two 20W blue LED spotlights $(5 \mathrm{~cm}$ away) for $15 \mathrm{~h}$. Upon reaction complete the crude mixture was poured into $5 \% \mathrm{H}_{2} \mathrm{SO}_{4}$ solution $(5 \mathrm{~mL})$ and extracted with EtOAc $(3 \times 2 \mathrm{~mL})$. The combined organic layers were washed with water $(2 \mathrm{~mL})$, saturated solution of $\mathrm{NaHCO}_{3}(2 \mathrm{~mL})$ and dried over $\mathrm{Na}_{2} \mathrm{SO}_{4}$. After removal of the solvent in vacuum, the residue was purified by flash column chromatography on silica gel using petroleum ether/EtOAc as the eluent to yield the corresponding products.

\section{General procedure B:}

A $4 \mathrm{~mL}$ vial equipped with a stirring bar was charged with cyclopropanol $(0.25$ mmol, 1 eq.), $\mathrm{NiCl}_{2} \cdot \mathrm{DME}$ (1.4 mg, $6.25 \mu \mathrm{mol}, 2.5 \mathrm{~mol} \%$ ), 2,9-neocuproine (1.3 $\mathrm{mg}$, $6.25 \mu \mathrm{mol}, 2.5 \mathrm{~mol} \%$ ), 4CzIPN (9.9 mg, $12.5 \mu \mathrm{mol}, 5 \mathrm{~mol} \%), \mathrm{K}_{2} \mathrm{CO}_{3}(103.5 \mathrm{mg}$, $0.75 \mathrm{mmol}, 3$ eq. $)$ and $\mathrm{Ti}(\mathrm{O} i \mathrm{Pr})_{4}(148 \mu \mathrm{L}, 0.50 \mathrm{mmol}, 2$ eq. $)$. The vial was purged with Ar for $5 \mathrm{~min}$ followed by addition of vinyl bromide $(0.50 \mathrm{mmol}, 2 \mathrm{eq})$ solution in acetone $(2.5 \mathrm{~mL})$. The resulting mixture was set to stir $(1200 \mathrm{rpm})$ and irradiated with two $20 \mathrm{~W}$ blue LED spotlights ( $5 \mathrm{~cm}$ away) for $15 \mathrm{~h}$. Upon reaction complete the crude mixture was poured into $5 \% \mathrm{H}_{2} \mathrm{SO}_{4}$ solution $(5 \mathrm{~mL})$ and extracted with EtOAc $(3 \times 2 \mathrm{~mL})$. The combined organic layers were washed with water $(2 \mathrm{~mL})$, saturated solution of $\mathrm{NaHCO}_{3}(2 \mathrm{~mL})$ and dried over $\mathrm{Na}_{2} \mathrm{SO}_{4}$. After removal of the solvent in vacuum, the residue was purified by flash column chromatography on silica gel using petroleum ether/EtOAc as the eluent to yield the corresponding products.

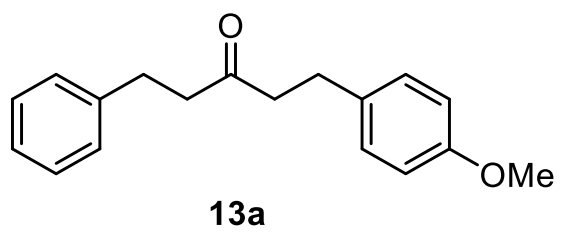

1-(4-methoxyphenyl)-5-phenylpentan-3-one (13a): Obtained according to the general procedure $A$ from cyclopropanol 11a and 4-bromoanisol. The crude product was purified by flash column chromatography on silica gel $(5 \% \mathrm{EtOAc} / \mathrm{PE})$ to provide a colorless oil ( $38.5 \mathrm{mg}, 0.144 \mathrm{mmol}, 58 \%$ yield). The yields of the product obtained from 4-methoxyphenyl trifluoromethanesulfonate and 1-chloro-4methoxybenzene were $18 \%$ and $0 \%$, respectively; ${ }^{1} \mathbf{H}$ NMR $\left(500 \mathrm{MHz}, \mathrm{CDCl}_{3}\right) \delta$ $7.28(\mathrm{~m}, 2 \mathrm{H}), 7.20(\mathrm{t}, J=7.4 \mathrm{~Hz}, 1 \mathrm{H}), 7.16(\mathrm{~d}, J=7.0 \mathrm{~Hz}, 2 \mathrm{H}), 7.08(\mathrm{~d}, J=8.7 \mathrm{~Hz}$, 
2H), $6.82(\mathrm{~d}, J=8.7 \mathrm{~Hz}, 2 \mathrm{H}), 3.79(\mathrm{~s}, 3 \mathrm{H}), 2.89(\mathrm{t}, J=7.6 \mathrm{~Hz}, 2 \mathrm{H}), 2.84(\mathrm{t}, J=7.6$ $\mathrm{Hz}, 2 \mathrm{H}), 2.71(\mathrm{t}, J=7.6 \mathrm{~Hz}, 1 \mathrm{H}), 2.68(\mathrm{t}, J=7.6 \mathrm{~Hz}, 1 \mathrm{H}) .{ }^{13} \mathbf{C}$ NMR $(126 \mathrm{MHz}$, $\left.\mathrm{CDCl}_{3}\right) \delta 209.4,158.1,141.2,133.2,129.4$ (2C), 128.6 (2C), 128.4 (2C), 126.2, 114.0 (2C), 55.4, 44.9, 44.7, 29.8, 29.0. HRMS (ESI+): $m / z$ calc'd for $\mathrm{C}_{18} \mathrm{H}_{20} \mathrm{NaO}_{2}$ $[\mathrm{M}+\mathrm{Na}]^{+}: 291.1356$, found 291.1364 .

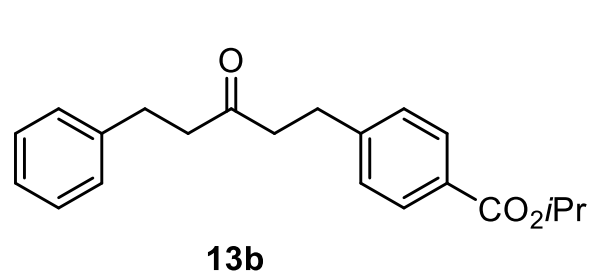

isopropyl 4-(3-oxo-5-phenylpentyl)benzoate

(13b): Obtained according to the general procedure A from cyclopropanol 11a and isopropyl 4bromobenzoate. The crude product was purified by flash column chromatography on silica gel $(3 \%$ EtOAc/PE) to provide a colorless oil (38.5 mg, $0.119 \mathrm{mmol}, 48 \%$ yield); ${ }^{1} \mathbf{H}$ NMR $\left(500 \mathrm{MHz}, \mathrm{CDCl}_{3}\right) \delta 7.94(\mathrm{~d}, J=8.3 \mathrm{~Hz}, 2 \mathrm{H}), 7.30-7.24(\mathrm{~m}, 2 \mathrm{H}), 7.23-7.18(\mathrm{~m}$, $3 \mathrm{H}), 7.17-7.13(\mathrm{~m}, 2 \mathrm{H}), 5.24$ (hept, $J=6.3 \mathrm{~Hz}, 1 \mathrm{H}), 2.93(\mathrm{t}, J=7.5 \mathrm{~Hz}, 2 \mathrm{H}), 2.89$ $(\mathrm{t}, J=7.5 \mathrm{~Hz}, 2 \mathrm{H}), 2.71(\mathrm{t}, J=7.5 \mathrm{~Hz}, 4 \mathrm{H}), 1.36(\mathrm{~d}, J=6.3 \mathrm{~Hz}, 6 \mathrm{H}) .{ }^{13} \mathbf{C}$ NMR $(126$ $\left.\mathrm{MHz}, \mathrm{CDCl}_{3}\right) \delta 208.7,166.1,146.4,141.0,129.9$ (2C), 129.0, 128.6 (2C), 128.4 (2C), 128.4 (2C), 126.3, 68.3, 44.6, 44.1, 29.8, 29.7, 22.1 (2C). HRMS (ESI+): $m / z$ calc'd for $\mathrm{C}_{21} \mathrm{H}_{24} \mathrm{NaO}_{3}[\mathrm{M}+\mathrm{Na}]^{+}: 347.1618$, found 347.1618 .

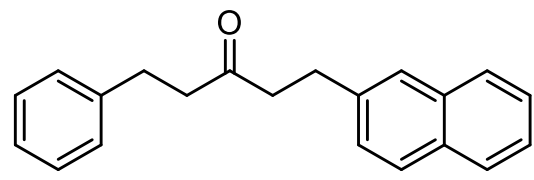

$13 c$ 1-(naphthalen-2-yl)-5-phenylpentan-3-one $\quad(13 \mathrm{c})$ : Obtained according to the general procedure A from cyclopropanol 11a and 2-bromonaphthalene. The crude product was purified by flash column chromatography on silica gel (3\% EtOAc/PE) to provide a colorless oil $(40.0 \mathrm{mg}, 0.139 \mathrm{mmol}, 56 \%$ yield $)$; ${ }^{1} \mathbf{H}$ NMR $(500 \mathrm{MHz}$, $\left.\mathrm{CDCl}_{3}\right) \delta 7.83-7.80(\mathrm{~m}, 1 \mathrm{H}), 7.77(\mathrm{~d}, J=8.2 \mathrm{~Hz}, 2 \mathrm{H}), 7.61(\mathrm{~s}, 1 \mathrm{H}), 7.50-7.41(\mathrm{~m}$, 2H), $7.31(\mathrm{dd}, J=8.4,1.7 \mathrm{~Hz}, 1 \mathrm{H}), 7.29-7.24(\mathrm{~m}, 2 \mathrm{H}), 7.22-7.17(\mathrm{~m}, 1 \mathrm{H}), 7.17$ $-7.13(\mathrm{~m}, 2 \mathrm{H}), 3.07$ (t, $J=7.6 \mathrm{~Hz}, 2 \mathrm{H}), 2.91(\mathrm{t}, J=7.6 \mathrm{~Hz}, 2 \mathrm{H}), 2.81(\mathrm{t}, J=7.6 \mathrm{~Hz}$, 2H), $2.74(\mathrm{t}, J=7.6 \mathrm{~Hz}, 2 \mathrm{H}) ;{ }^{13} \mathbf{C}$ NMR $\left(126 \mathrm{MHz}, \mathrm{CDCl}_{3}\right) \delta 209.2,141.1,138.6$, $133.7,132.2,128.6$ (2C), 128.4 (2C), 128.2, 127.7, 127.6, 127.2, 126.6, 126.2, 126.1, 125.5, 44.7, 44.5, 30.0, 29.9. HRMS (ESI+): $\mathrm{m} / z$ calc'd for $\mathrm{C}_{21} \mathrm{H}_{20} \mathrm{NaO}$ $[\mathrm{M}+\mathrm{Na}]^{+}: 311.1406$, found 311.1400 .

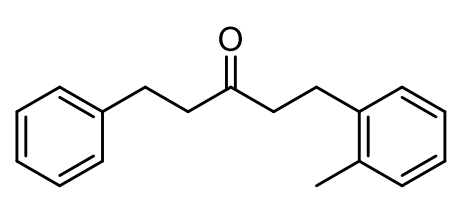

$13 d$

1-phenyl-5-(o-tolyl)pentan-3-one

(13d): Obtained according to the general procedure A from cyclopropanol 11a and 1-bromo-2-methylbenzene. The crude product was purified by flash column chromatography on silica gel (3\% EtOAc/PE) to provide a colorless oil $(28.9 \mathrm{mg}, 0.115$ mmol, $46 \%$ yield); ${ }^{1} \mathbf{H}$ NMR $\left(500 \mathrm{MHz}, \mathrm{CDCl}_{3}\right) \delta 7.32-7.26(\mathrm{~m}, 2 \mathrm{H}), 7.22-7.16$ 
(m, 3H), $7.16-7.07(\mathrm{~m}, 4 \mathrm{H}), 2.91(\mathrm{t}, \mathrm{J}=7.6 \mathrm{~Hz}, 2 \mathrm{H}), 2.88(\mathrm{t}, \mathrm{J}=7.9 \mathrm{~Hz}, 2 \mathrm{H}), 2.74$ $(\mathrm{t}, \mathrm{J}=7.6 \mathrm{~Hz}, 2 \mathrm{H}), 2.66(\mathrm{t}, \mathrm{J}=7.9 \mathrm{~Hz}, 2 \mathrm{H}), 2.29$ (s, 3H). ${ }^{13} \mathbf{C} \mathbf{~ N M R}(126 \mathrm{MHz}$, $\left.\mathrm{CDCl}_{3}\right) \delta 209.4,141.1,139.2,136.0,135.7,130.4,128.7,128.6(2 \mathrm{C}), 128.4(2 \mathrm{C})$, 126.4, 126.3, 44.6, 43.3, 30.0, 27.2, 19.4. HRMS (ESI+): $m / z$ calc'd for $\mathrm{C}_{18} \mathrm{H}_{20} \mathrm{NaO}$ $[\mathrm{M}+\mathrm{Na}]^{+}: 275.1406$, found 275.1410 .

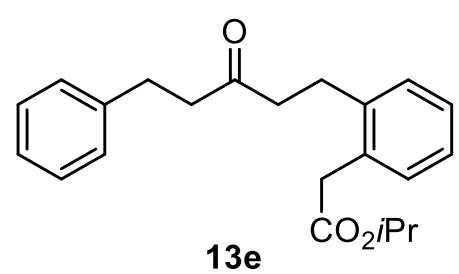

isopropyl 2-(2-(3-oxo-5-phenylpentyl)phenyl)acetate (13e): Obtained according to the general procedure A from cyclopropanol 11a and 1-methylethyl 2bromobenzeneacetate. The crude product was purified by flash column chromatography on silica gel (6\% EtOAc/PE) to provide a colorless oil (43.7 mg, $0.129 \mathrm{mmol}, 52 \%$ yield); ${ }^{1} \mathbf{H}$ NMR (500 MHz, $\left.\mathrm{CDCl}_{3}\right) \delta 7.30-7.27(\mathrm{~m}, 1 \mathrm{H}), 7.23-7.11(\mathrm{~m}, 8 \mathrm{H}), 4.99$ (hept, $\left.J=6.3 \mathrm{~Hz}, 1 \mathrm{H}\right), 3.61$ (s, 2H), $2.93-2.87(\mathrm{~m}, 4 \mathrm{H}), 2.75-2.68(\mathrm{~m}, 4 \mathrm{H}), 1.22(\mathrm{~d}, J=6.3 \mathrm{~Hz}, 6 \mathrm{H}) .{ }^{13} \mathbf{C}$ NMR $\left(126 \mathrm{MHz}, \mathrm{CDCl}_{3}\right) \delta 209.1,171.3,141.1,139.7,132.7,130.7,129.4,128.6$ (2C), 128.4 (2C), 127.7, 126.6, 126.3, 68.4, 44.6, 44.0, 39.0, 29.9, 26.8, 21.9 (2C). HRMS (ESI+): $m / z$ calc'd for $\mathrm{C}_{22} \mathrm{H}_{26} \mathrm{NaO}_{3}[\mathrm{M}+\mathrm{Na}]^{+}: 361.1774$, found 361.1779 .

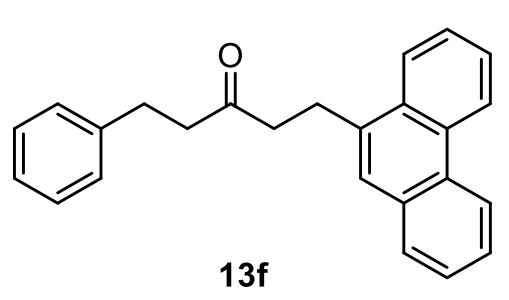

\section{1-(phenanthren-9-yl)-5-phenylpentan-3-one}

(13f):

Obtained according to the general procedure A from cyclopropanol 11a and 9-bromophenanthrene. The crude product was purified by flash column chromatography on silica gel ( $3 \%$ EtOAc/PE) to provide a colorless oil $(42.7$ mg, 0.126 mmol, $51 \%$ yield); ${ }^{1} \mathbf{H}$ NMR $\left(500 \mathrm{MHz}, \mathrm{CDCl}_{3}\right) \delta 8.75$ (dd, J = 8.4, 0.9 $\mathrm{Hz}, 1 \mathrm{H}), 8.66(\mathrm{~d}, \mathrm{~J}=8.0 \mathrm{~Hz}, 1 \mathrm{H}), 8.02(\mathrm{dd}, \mathrm{J}=8.0,1.3 \mathrm{~Hz}, 1 \mathrm{H}), 7.82(\mathrm{dd}, \mathrm{J}=7.8$, $1.3 \mathrm{~Hz}, 1 \mathrm{H}), 7.71-7.58(\mathrm{~m}, 4 \mathrm{H}), 7.57(\mathrm{~s}, 1 \mathrm{H}), 7.28(\mathrm{t}, \mathrm{J}=7.5 \mathrm{~Hz}, 2 \mathrm{H}), 7.20(\mathrm{t}, \mathrm{J}=$ $7.5 \mathrm{~Hz}, 1 \mathrm{H}), 7.16(\mathrm{~d}, \mathrm{~J}=7.0 \mathrm{~Hz}, 2 \mathrm{H}), 3.40(\mathrm{t}, \mathrm{J}=8.3 \mathrm{~Hz}, 2 \mathrm{H}), 2.93(\mathrm{t}, \mathrm{J}=7.6 \mathrm{~Hz}$, 2H), 2.89 (t, J = 8.3 Hz, 2H), $2.75(\mathrm{t}, \mathrm{J}=7.6 \mathrm{~Hz}, 2 \mathrm{H}) .{ }^{13} \mathbf{C}$ NMR $\left(126 \mathrm{MHz}, \mathrm{CDCl}_{3}\right)$

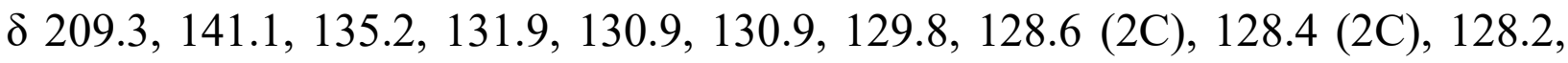
126.8 (2C), 126.5, 126.4, 126.3, 126.2, 124.2, 123.5, 122.6, 44.6, 43.4, 30.0, 27.2. HRMS (ESI+): $m / z$ calc'd for $\mathrm{C}_{25} \mathrm{H}_{22} \mathrm{NaO}[\mathrm{M}+\mathrm{Na}]^{+}: 361.1563$, found 361.1562 .

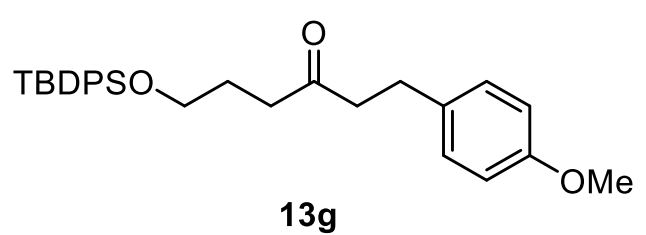

6-((tert-butyldiphenylsilyl)oxy)-1-(4methoxyphenyl)hexane-3-one (13g): Obtained according to the general procedure A from cyclopropanol 11b and 4-bromoanisol. The crude product was purified by flash column chromatography on silica gel (3\% EtOAc/PE) to provide a colorless oil $(63.0 \mathrm{mg}$, $0.137 \mathrm{mmol}, 55 \%$ yield $) ;{ }^{1} \mathbf{H}$ NMR $\left(500 \mathrm{MHz}, \mathrm{CDCl}_{3}\right) \delta 7.67-7.63(\mathrm{~m}, 4 \mathrm{H}), 7.45$ 
$-7.36(\mathrm{~m}, 6 \mathrm{H}), 7.10(\mathrm{~d}, \mathrm{~J}=8.6 \mathrm{~Hz}, 2 \mathrm{H}), 6.83(\mathrm{~d}, \mathrm{~J}=8.6 \mathrm{~Hz}, 2 \mathrm{H}), 3.78(\mathrm{~s}, 3 \mathrm{H}), 3.66$ $(\mathrm{t}, \mathrm{J}=6.1 \mathrm{~Hz}, 2 \mathrm{H}), 2.84(\mathrm{t}, \mathrm{J}=7.6 \mathrm{~Hz}, 2 \mathrm{H}), 2.70(\mathrm{t}, \mathrm{J}=7.6 \mathrm{~Hz}, 2 \mathrm{H}), 2.51(\mathrm{t}, \mathrm{J}=7.3$ $\mathrm{Hz}, 2 \mathrm{H}), 1.87-1.78(\mathrm{~m}, 2 \mathrm{H}), 1.05(\mathrm{~s}, 9 \mathrm{H}) .{ }^{13} \mathbf{C}$ NMR $\left(126 \mathrm{MHz}, \mathrm{CDCl}_{3}\right) \delta 210.2$, 158.1, 135.7 (4C), 133.9 (2C), 133.3, 129.8 (2C), 129.4 (2C), 127.8 (4C), 114.0 (2C), 63.1, 55.4, 44.7, 39.5, 29.1, 27.0 (3C), 26.7, 19.3. HRMS (ESI+): $m / z$ calc'd for $\mathrm{C}_{29} \mathrm{H}_{36} \mathrm{NaO}_{3} \mathrm{Si}[\mathrm{M}+\mathrm{Na}]^{+}:$483.2326, found 483.2321 .

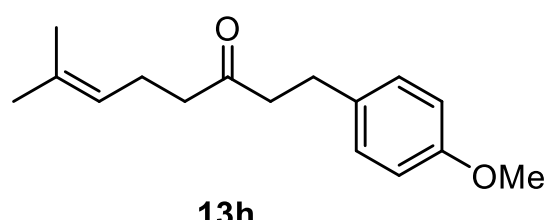

$13 h$

1-(4-methoxyphenyl)-7-methyloct-6-en-3-one (13h): Obtained according to the general procedure A from cyclopropanol 11c and 4-bromoanisol. The crude product was purified by flash column chromatography on silica gel $(2 \% \mathrm{EtOAc} / \mathrm{PE})$ to provide a colorless oil $(27.5 \mathrm{mg}, 0.112 \mathrm{mmol}, 45 \%$ yield $) ;{ }^{1} \mathbf{H}$ NMR $(500 \mathrm{MHz}$, $\left.\mathrm{CDCl}_{3}\right) \delta 7.09(\mathrm{~d}, \mathrm{~J}=8.6 \mathrm{~Hz}, 2 \mathrm{H}), 6.82(\mathrm{~d}, \mathrm{~J}=8.6 \mathrm{~Hz}, 2 \mathrm{H}), 5.06-5.00(\mathrm{~m}, 1 \mathrm{H})$, $3.78(\mathrm{~s}, 3 \mathrm{H}), 2.84(\mathrm{t}, \mathrm{J}=7.6 \mathrm{~Hz}, 2 \mathrm{H}), 2.69(\mathrm{t}, \mathrm{J}=7.6 \mathrm{~Hz}, 2 \mathrm{H}), 2.40(\mathrm{t}, \mathrm{J}=7.4 \mathrm{~Hz}$, 2H), $2.28-2.19(\mathrm{~m}, 2 \mathrm{H}), 1.67$ (s, 3H), 1.60 (s, 3H). ${ }^{13} \mathbf{C ~ N M R}\left(126 \mathrm{MHz}, \mathrm{CDCl}_{3}\right) \delta$ 210.2, 158.1, 133.3, 129.4 (2C), 127.8, 122.9, 114.0 (2C), 55.4, 44.7, 43.1, 29.1, 25.8, 22.6, 17.8. HRMS (ESI+): $m / z$ calc'd for $\mathrm{C}_{16} \mathrm{H}_{22} \mathrm{NaO}_{2}[\mathrm{M}+\mathrm{Na}]^{+}:$269.1512, found 269.1512 .

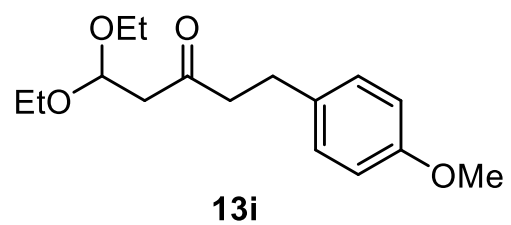

\section{1,1-diethoxy-5-(4-methoxyphenyl)pentan-3-one} (13i): Obtained according to the general procedure A from cyclopropanol 11d and 4-bromoanisol. Upon reaction complete the crude mixture was poured into 5 $\mathrm{mL}$ saturated aqueous solution of $\mathrm{NH}_{4} \mathrm{Cl}$ and extracted with EtOAc $(3 \times 2 \mathrm{~mL})$. The crude product was purified by flash column chromatography on silica gel (5\% EtOAc/PE) to provide a colorless oil $(30.1 \mathrm{mg}$, $0.107 \mathrm{mmol}, 43 \%$ yield); ${ }^{1} \mathrm{H}$ NMR $\left(500 \mathrm{MHz}, \mathrm{CDCl}_{3}\right) \delta 7.09(\mathrm{~d}, \mathrm{~J}=8.6 \mathrm{~Hz}, 2 \mathrm{H})$, $6.81(\mathrm{~d}, \mathrm{~J}=8.6 \mathrm{~Hz}, 2 \mathrm{H}), 4.89(\mathrm{t}, \mathrm{J}=5.7 \mathrm{~Hz}, 1 \mathrm{H}), 3.78(\mathrm{~s}, 3 \mathrm{H}), 3.65(\mathrm{dq}, \mathrm{J}=9.3,7.1$ $\mathrm{Hz}, 2 \mathrm{H}), 3.50(\mathrm{dq}, \mathrm{J}=9.3,7.1 \mathrm{~Hz}, 2 \mathrm{H}), 2.86-2.80(\mathrm{~m}, 2 \mathrm{H}), 2.78-2.73(\mathrm{~m}, 2 \mathrm{H})$, $2.72(\mathrm{~d}, \mathrm{~J}=5.7 \mathrm{~Hz}, 2 \mathrm{H}), 1.17(\mathrm{t}, \mathrm{J}=7.0 \mathrm{~Hz}, 6 \mathrm{H}) .{ }^{13} \mathrm{C} \mathrm{NMR}\left(126 \mathrm{MHz}, \mathrm{CDCl}_{3}\right) \delta$ 207.2, 158.1, 133.2, 129.4 (2C), 114.0 (2C), 100.1, 62.5 (2C), 55.4, 47.8, 45.9, 28.6, 15.4 (2C). HRMS (ESI+): $m / z$ calc'd for $\mathrm{C}_{16} \mathrm{H}_{24} \mathrm{NaO}_{4}[\mathrm{M}+\mathrm{Na}]^{+}: 303.1567$, found 303.1561 .

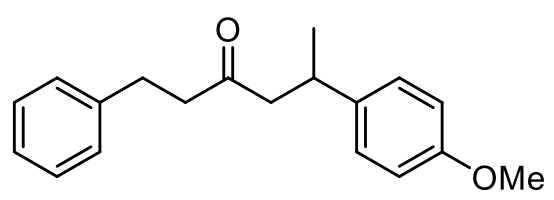

13j

\section{5-(4-methoxyphenyl)-1-phenylhexan-3-one (13j):}

Obtained according to the general procedure A from cyclopropanol 11e and 4-bromoanisol. The crude product was purified by flash column chromatography 
on silica gel (2\% EtOAc/PE) to provide a colorless oil $(33.9 \mathrm{mg}, 0.120 \mathrm{mmol}, 48 \%$ yield); ${ }^{1} \mathbf{H}$ NMR $\left(500 \mathrm{MHz}, \mathrm{CDCl}_{3}\right) \delta 7.23-7.28(\mathrm{~m}, 2 \mathrm{H}), 7.22-7.16(\mathrm{~m}, 1 \mathrm{H}), 7.13$ $-7.08(\mathrm{~m}, 4 \mathrm{H}), 6.83(\mathrm{~d}, \mathrm{~J}=8.7 \mathrm{~Hz}, 2 \mathrm{H}), 3.79(\mathrm{~s}, 3 \mathrm{H}), 3.27$ (h, J = 7.0 Hz, 1H), 2.85 $-2.79(\mathrm{~m}, 2 \mathrm{H}), 2.71-2.55(\mathrm{~m}, 4 \mathrm{H}), 1.22(\mathrm{~d}, \mathrm{~J}=7.0 \mathrm{~Hz}, 3 \mathrm{H}) .{ }^{13} \mathbf{C} \mathbf{N M R}(126 \mathrm{MHz}$, $\left.\mathrm{CDCl}_{3}\right) \delta 209.2,158.2,141.2,138.3,128.6(2 \mathrm{C}), 128.4(2 \mathrm{C}), 127.8(2 \mathrm{C}), 126.2$, 114.0 (2C), 55.4, 51.8, 45.1, 34.9, 29.7, 22.3. HRMS (ESI+): $\mathrm{m} / \mathrm{z}$ calc'd for $\mathrm{C}_{19} \mathrm{H}_{22} \mathrm{NaO}_{2}[\mathrm{M}+\mathrm{Na}]^{+}:$305.1512, found 305.1526.

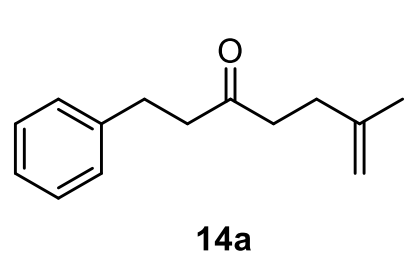

6-methyl-1-phenylhept-6-en-3-one (14a): Obtained according to the general procedure $B$ from cyclopropanol 11a and 2-bromoprop-1-ene. The crude product was purified by flash column chromatography on silica gel (1\% EtOAc/PE) to provide a colorless oil $(42.5 \mathrm{mg}, 0.210 \mathrm{mmol}, 84 \%$ yield $)$; ${ }^{1} \mathbf{H}$ NMR $\left(500 \mathrm{MHz}, \mathrm{CDCl}_{3}\right) \delta 7.31-7.25(\mathrm{~m}, 2 \mathrm{H}), 7.22-7.15(\mathrm{~m}, 3 \mathrm{H}), 4.72(\mathrm{~s}$, $1 \mathrm{H}), 4.63(\mathrm{~s}, 1 \mathrm{H}), 2.94-2.87(\mathrm{~m}, 2 \mathrm{H}), 2.79-2.73(\mathrm{~m}, 2 \mathrm{H}), 2.57-2.50(\mathrm{~m}, 2 \mathrm{H})$, $2.31-2.24(\mathrm{~m}, 2 \mathrm{H}), 1.71(\mathrm{~s}, 3 \mathrm{H}) .{ }^{13} \mathbf{C}$ NMR $\left(126 \mathrm{MHz}, \mathrm{CDCl}_{3}\right) \delta$ 209.6, 144.6, 141.2, 128.6, 128.5, 126.2, 110.3, 44.4, 41.3, 31.5, 29.9, 22.8. HRMS (ESI+): $\mathrm{m} / \mathrm{z}$ calc'd for $\mathrm{C}_{14} \mathrm{H}_{19} \mathrm{O}[\mathrm{M}+\mathrm{H}]^{+}:$203.1430, found 203.1433.

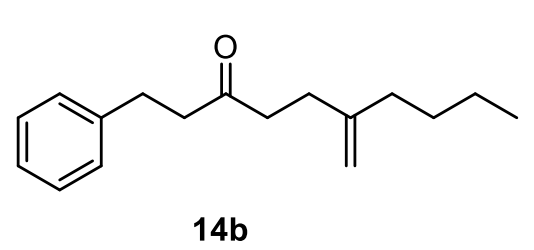

6-methylene-1-phenyldecan-3-one (14b): Obtained according to the general procedure $\mathrm{B}$ from cyclopropanol 11a and 2-bromohex-1-ene. The crude product was purified by flash column chromatography on silica gel $(1 \rightarrow 1.5 \%$ EtOAc/PE) to provide a colorless oil (33.6 mg, $0.137 \mathrm{mmol}, 55 \%$ yield); ${ }^{1} \mathbf{H}$ NMR (500 MHz, $\left.\mathrm{CDCl}_{3}\right) \delta 7.31-7.25$ $(\mathrm{m}, 2 \mathrm{H}), 7.22-7.17(\mathrm{~m}, 3 \mathrm{H}), 4.72(\mathrm{~s}, 1 \mathrm{H}), 4.65(\mathrm{~s}, 1 \mathrm{H}), 2.93-2.88(\mathrm{~m}, 2 \mathrm{H}), 2.78-$ $2.73(\mathrm{~m}, 2 \mathrm{H}), 2.56-2.50(\mathrm{~m}, 2 \mathrm{H}), 2.31-2.24(\mathrm{~m}, 2 \mathrm{H}), 2.03-1.97(\mathrm{~m}, 2 \mathrm{H}), 1.43-$ $1.36(\mathrm{~m}, 2 \mathrm{H}), 1.35-1.25(\mathrm{~m}, 2 \mathrm{H}), 0.90(\mathrm{t}, J=7.3 \mathrm{~Hz}, 3 \mathrm{H}) .{ }^{13} \mathbf{C}$ NMR $(126 \mathrm{MHz}$, $\left.\mathrm{CDCl}_{3}\right) \delta 209.7,148.8,141.3,128.6,128.5,126.2,109.1,44.5,41.5,36.2,30.1$, 29.9, 29.8, 22.6, 14.1. HRMS (ESI+): $m / z$ calc'd for $\mathrm{C}_{17} \mathrm{H}_{25} \mathrm{O}[\mathrm{M}+\mathrm{H}]^{+}:$245.1900, found 245.1901 .

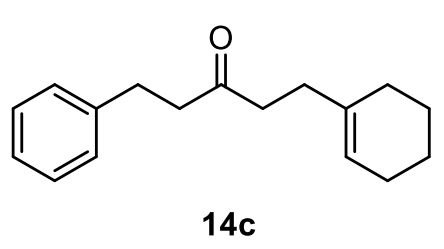

1-(cyclohex-1-en-1-yl)-5-phenylpentan-3-one

(14c): Obtained according to the general procedure B from cyclopropanol 11a and cyclohex-1-en-1-yl trifluoromethanesulfonate. The crude product was purified by flash column chromatography on silica gel (1\% EtOAc/PE) to provide a colorless oil (36.4 mg, $0.150 \mathrm{mmol}, 60 \%$ yield); ${ }^{1} \mathbf{H}$ NMR $\left(500 \mathrm{MHz}, \mathrm{CDCl}_{3}\right) \delta 7.31-7.25(\mathrm{~m}, 2 \mathrm{H}), 7.22-7.16(\mathrm{~m}, 3 \mathrm{H}), 5.39-5.33(\mathrm{~m}, 1 \mathrm{H})$, 
$2.90(\mathrm{t}, J=7.6 \mathrm{~Hz}, 2 \mathrm{H}), 2.74(\mathrm{t}, J=7.6 \mathrm{~Hz}, 2 \mathrm{H}), 2.52-2.46(\mathrm{~m}, 2 \mathrm{H}), 2.23-2.15$ (m, 2H), $1.99-1.92(\mathrm{~m}, 2 \mathrm{H}), 1.92-1.86(\mathrm{~m}, 2 \mathrm{H}), 1.64-1.57(\mathrm{~m}, 2 \mathrm{H}), 1.56-1.50$ (m, 2H). ${ }^{13} \mathbf{C}$ NMR $\left(126 \mathrm{MHz}, \mathrm{CDCl}_{3}\right) \delta 210.1,141.3,136.4,128.6,128.5,126.2$, $121.5,44.4,41.5,32.0,29.9,28.5,25.3,23.0,22.5$. HRMS (ESI +$): m / z$ calc'd for $\mathrm{C}_{17} \mathrm{H}_{23} \mathrm{O}[\mathrm{M}+\mathrm{H}]^{+}:$243.1743, found 243.1746.

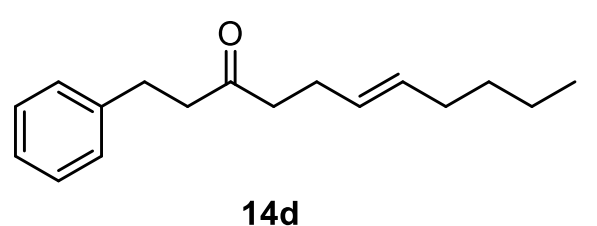

to provide a colorless oil (27.5 mg, $0.113 \mathrm{mmol}, 45 \%$ yield); ${ }^{1} \mathbf{H}$ NMR $(500 \mathrm{MHz}$, $\left.\mathrm{CDCl}_{3}\right) \delta 7.31-7.25(\mathrm{~m}, 2 \mathrm{H}), 7.22-7.15(\mathrm{~m}, 3 \mathrm{H}), 5.47-5.31(\mathrm{~m}, 2 \mathrm{H}), 2.93-2.87$ $(\mathrm{m}, 2 \mathrm{H}), 2.76-2.70(\mathrm{~m}, 2 \mathrm{H}), 2.47-2.42(\mathrm{~m}, 2 \mathrm{H}), 2.28-2.22(\mathrm{~m}, 2 \mathrm{H}), 2.00-1.92$ $(\mathrm{m}, 2 \mathrm{H}), 1.34-1.24(\mathrm{~m}, 4 \mathrm{H}), 0.88(\mathrm{t}, J=7.0 \mathrm{~Hz}, 3 \mathrm{H}) .{ }^{13} \mathbf{C} \mathbf{~ N M R}\left(126 \mathrm{MHz}, \mathrm{CDCl}_{3}\right)$ $\delta$ 209.7, 141.3, 131.8, 128.6, 128.4, 128.3, 126.2, 44.5, 43.0, 32.3, 31.8, 29.8, 27.0, 22.3, 14.1. HRMS (ESI+): $m / z$ calc'd for $\mathrm{C}_{17} \mathrm{H}_{25} \mathrm{O}[\mathrm{M}+\mathrm{H}]^{+}: 245.1900$, found 245.1899 .

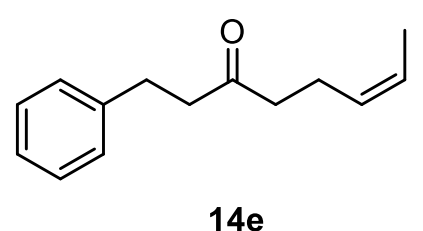

(Z)-1-phenyloct-6-en-3-one (14e): Obtained according to the general procedure A from cyclopropanol 11a and $(Z)-1-$ bromoprop-1-ene. The crude product was purified by flash column chromatography on silica gel $(2 \%$ EtOAc/PE) to provide a colorless oil as 1:8 mixture of $E / Z$ isomers $(23.2$ mg, 0.115 mmol, 46\% yield); ${ }^{1} \mathrm{H}$ NMR (500 MHz, $\left.\mathrm{CDCl}_{3}\right) \delta 7.31-7.25(\mathrm{~m}, 2 \mathrm{H})$, $7.22-7.15(\mathrm{~m}, 3 \mathrm{H}), 5.51-5.27(\mathrm{~m}, 2 \mathrm{H}), 2.90(\mathrm{t}, \mathrm{J}=7.7 \mathrm{~Hz}, 2 \mathrm{H}), 2.73(\mathrm{t}, \mathrm{J}=7.7$ $\mathrm{Hz}, 2 \mathrm{H}), 2.44(\mathrm{t}, \mathrm{J}=7.4 \mathrm{~Hz}, 2 \mathrm{H}), 2.35-2.27(\mathrm{~m}, 2 \mathrm{H}), 1.62-1.58(\mathrm{~m}, 3 \mathrm{H}) .{ }^{13} \mathrm{C}$ NMR $\left(126 \mathrm{MHz}, \mathrm{CDCl}_{3}\right) \delta 209.8,141.3,128.8,128.6(2 \mathrm{C}), 128.5$ (2C), 126.2, 125.2, 44.5, 42.8, 29.9, 21.4, 12.8. HRMS (ESI+): $\mathrm{m} / z$ calc'd for $\mathrm{C}_{14} \mathrm{H}_{19} \mathrm{O}[\mathrm{M}+\mathrm{H}]^{+}:$203.1430, found 203.1431 .

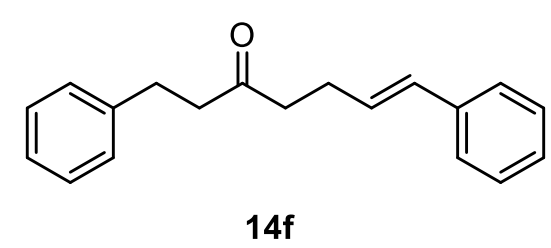

(E)-1,7-diphenylhept-6-en-3-one (14f): Obtained according to the general procedure $\mathrm{B}$ from cyclopropanol 11a and (E)-(2-bromovinyl)benzene. The crude product was purified by flash column chromatography on silica gel $(1 \rightarrow 2 \%$ EtOAc/PE) to provide a colorless oil $(51.6 \mathrm{mg}, 0.195 \mathrm{mmol}, 78 \%$ yield $) ;{ }^{1} \mathbf{H}$ NMR $(500 \mathrm{MHz}$, $\left.\mathrm{CDCl}_{3}\right) \delta 7.34-7.24(\mathrm{~m}, 6 \mathrm{H}), 7.19(\mathrm{t}, J=7.4 \mathrm{~Hz}, 4 \mathrm{H}), 6.39(\mathrm{~d}, J=15.9 \mathrm{~Hz}, 1 \mathrm{H})$, $6.17(\mathrm{dt}, J=15.9,6.9 \mathrm{~Hz}, 1 \mathrm{H}), 2.92(\mathrm{t}, J=7.6 \mathrm{~Hz}, 2 \mathrm{H}), 2.76(\mathrm{t}, J=7.6 \mathrm{~Hz}, 2 \mathrm{H}), 2.57$ 
$(\mathrm{t}, J=7.2 \mathrm{~Hz}, 2 \mathrm{H}), 2.51-2.45(\mathrm{~m}, 2 \mathrm{H}) .{ }^{13} \mathbf{C} \mathbf{N M R}\left(126 \mathrm{MHz}, \mathrm{CDCl}_{3}\right) \delta 209.3,141.2$, $137.5,130.9,129.0,128.6,128.5,127.2,126.3,126.2,44.6,42.6,29.9,27.2$. HRMS (ESI+): $m / z$ calc'd for $\mathrm{C}_{19} \mathrm{H}_{21} \mathrm{O}[\mathrm{M}+\mathrm{H}]^{+}: 265.1587$, found 265.1590 .

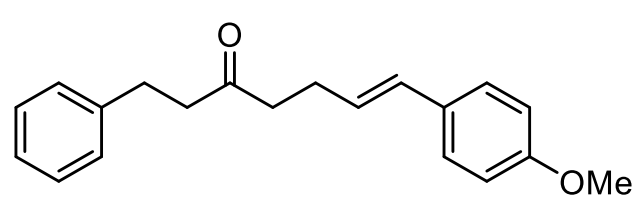

149

(E)-7-(4-methoxyphenyl)-1-phenylhept-6-en3-one (14g): Obtained according to the general procedure B from cyclopropanol 11a and $(E)-1-$ (2-bromovinyl)-4-methoxybenzene. The crude product was purified by flash column chromatography on silica gel (3\% EtOAc/PE) to provide a colorless oil as $6: 1$ mixture of $E / Z$ isomers (30.2 $\mathrm{mg}, 0.103 \mathrm{mmol}, 41 \%$ yield); ${ }^{1} \mathbf{H}$ NMR $(500 \mathrm{MHz}$, $\left.\mathrm{CDCl}_{3}\right) \delta 7.31-7.24(\mathrm{~m}, 5 \mathrm{H}), 7.20-7.17(\mathrm{~m}, 2 \mathrm{H}), 6.86-6.82(\mathrm{~m}, 2 \mathrm{H}), 6.34(\mathrm{~d}, \mathrm{~J}$ $=15.8 \mathrm{~Hz}, 1 \mathrm{H}), 6.03(\mathrm{dt}, \mathrm{J}=15.8,6.9 \mathrm{~Hz}, 1 \mathrm{H}), 3.80(\mathrm{~s}, 3 \mathrm{H}), 2.92(\mathrm{t}, \mathrm{J}=7.6 \mathrm{~Hz}, 2 \mathrm{H})$, $2.76(\mathrm{t}, \mathrm{J}=7.6 \mathrm{~Hz}, 2 \mathrm{H}), 2.56(\mathrm{t}, \mathrm{J}=7.3 \mathrm{~Hz}, 2 \mathrm{H}), 2.50-2.42(\mathrm{~m}, 2 \mathrm{H}) .{ }^{13} \mathbf{C}$ NMR (126 $\left.\mathrm{MHz}, \mathrm{CDCl}_{3}\right) \delta 209.4,159.0,141.2,130.4,130.3,128.6$ (2C), $128.4(2 \mathrm{C}), 127.2$ (2C), 126.7, 126.2, 114.1 (2C), 55.4, 44.5, 42.8, 29.9, 27.2. HRMS (ESI+): $m / z$ calc'd for $\mathrm{C}_{20} \mathrm{H}_{22} \mathrm{NaO}_{2}[\mathrm{M}+\mathrm{Na}]^{+}:$305.1512, found 305.1512 .

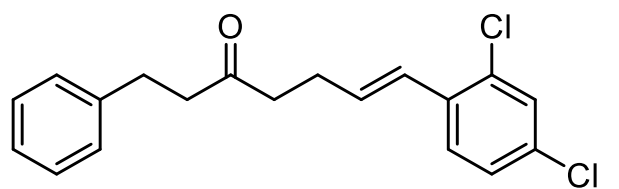

$14 h$

(E)-7-(2,4-dichlorophenyl)-1-phenylhept-6-en3-one (14h): Obtained according to the general procedure B from cyclopropanol 11a and $(E)-1-(2-$ bromovinyl)-2,4-dichlorobenzene. The crude product was purified by flash column chromatography on silica gel $(3 \%$ EtOAc/PE) to provide a colorless oil as 6:1 mixture of $E / Z$ isomers $\left(50.8 \mathrm{mg}, 0.153 \mathrm{mmol}, 61 \%\right.$ yield); ${ }^{1} \mathbf{H}$ NMR $(500 \mathrm{MHz}$, $\left.\mathrm{CDCl}_{3}\right) \delta 7.38(\mathrm{~d}, \mathrm{~J}=8.4 \mathrm{~Hz}, 1 \mathrm{H}), 7.34(\mathrm{~d}, \mathrm{~J}=2.1 \mathrm{~Hz}, 1 \mathrm{H}), 7.30-7.25(\mathrm{~m}, 3 \mathrm{H})$, $7.20-7.17(\mathrm{~m}, 3 \mathrm{H}), 6.68(\mathrm{~d}, \mathrm{~J}=15.8 \mathrm{~Hz}, 1 \mathrm{H}), 6.15(\mathrm{dt}, \mathrm{J}=15.8,6.8 \mathrm{~Hz}, 1 \mathrm{H}), 2.92$ $(\mathrm{t}, \mathrm{J}=7.6 \mathrm{~Hz}, 2 \mathrm{H}), 2.77$ (t, J = 7.6 Hz, 2H), 2.59 (t, J = 7.4 Hz, 2H), $2.55-2.48$ (m, 2H). ${ }^{13} \mathrm{C}$ NMR $\left(126 \mathrm{MHz}, \mathrm{CDCl}_{3}\right) \delta 209.0,141.1,134.2,133.2,133.0,132.6,129.4$, 128.6 (2C), 128.4 (2C), 127.6, 127.3, 126.3, 126.1, 44.5, 42.3, 29.9, 27.3. HRMS (ESI+): $m / z$ calc'd for $\mathrm{C}_{19} \mathrm{H}_{19} \mathrm{Cl}_{2} \mathrm{O}[\mathrm{M}+\mathrm{H}]^{+}: 333.0807$, found 333.0804 .

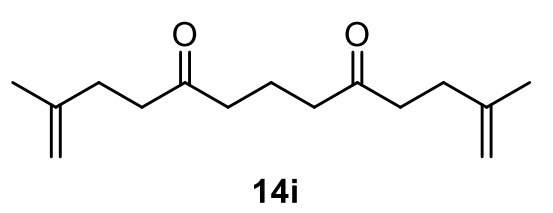

\section{2,12-dimethyltrideca-1,12-diene-5,9-dione}

(14i):

Obtained according to the general procedure B from cyclopropanol 11f, and 2-bromoprop-1-ene (4 eq.) with $\mathrm{NiCl}_{2} \cdot \mathrm{DME}(5 \%), 2,9$-neocuproine (5\%), 4CzIPN $(10 \%), \mathrm{Ti}(\mathrm{OiPr})_{4}$ (4 eq.), $\mathrm{K}_{2} \mathrm{CO}_{3}$ (6 eq.). The crude product was purified by flash column chromatography on silica gel $(5 \rightarrow 6 \%$ EtOAc/PE) to provide a colorless oil (30.1 mg, $0.127 \mathrm{mmol}, 51 \%$ yield); ${ }^{1} \mathbf{H}$ NMR $\left(500 \mathrm{MHz}, \mathrm{CDCl}_{3}\right) \delta 4.71(\mathrm{~s}, 2 \mathrm{H}), 4.63$ 
(s, 2H), $2.56-2.48(\mathrm{~m}, 4 \mathrm{H}), 2.45(\mathrm{t}, J=7.1 \mathrm{~Hz}, 4 \mathrm{H}), 2.26(\mathrm{t}, J=7.6 \mathrm{~Hz}, 4 \mathrm{H}), 1.84$ $(\mathrm{p}, J=7.1 \mathrm{~Hz}, 2 \mathrm{H}), 1.72(\mathrm{~s}, 6 \mathrm{H}) .{ }^{13} \mathbf{C}$ NMR $\left(126 \mathrm{MHz}, \mathrm{CDCl}_{3}\right) \delta 210.1,144.6,110.3$, 41.7, 41.0, 31.6, 22.7, 17.8. HRMS (ESI+): $m / z$ calc'd for $\mathrm{C}_{15} \mathrm{H}_{24} \mathrm{NaO}_{2}[\mathrm{M}+\mathrm{Na}]^{+}$: 259.1669 , found 259.1665 .

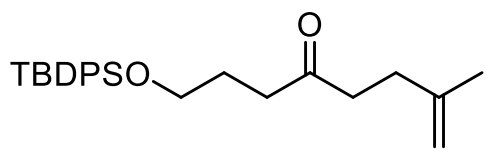

14j

\section{1-((tert-butyldiphenylsilyl)oxy)-7-methyloct-7-en-4-} one (14j): Obtained according to the general procedure B from cyclopropanol 11b and 2-bromoprop-1-ene. The crude product was purified by flash column chromatography on silica gel $(2 \% \mathrm{EtOAc} / \mathrm{PE})$ to provide a colorless oil $(74.0 \mathrm{mg}$, $0.188 \mathrm{mmol}, 75 \%$ yield); ${ }^{1} \mathbf{H}$ NMR $\left(500 \mathrm{MHz}, \mathrm{CDCl}_{3}\right) \delta 7.68-7.62(\mathrm{~m}, 4 \mathrm{H}), 7.46$ $-7.34(\mathrm{~m}, 6 \mathrm{H}), 4.73(\mathrm{~s}, 1 \mathrm{H}), 4.65(\mathrm{~s}, 1 \mathrm{H}), 3.67(\mathrm{t}, J=5.9 \mathrm{~Hz}, 2 \mathrm{H}), 2.58-2.50(\mathrm{~m}$, 4H), $2.27(\mathrm{t}, J=7.5 \mathrm{~Hz}, 2 \mathrm{H}), 1.88-1.78(\mathrm{~m}, 2 \mathrm{H}), 1.73(\mathrm{~s}, 3 \mathrm{H}), 1.05(\mathrm{~s}, 9 \mathrm{H}) .{ }^{13} \mathrm{C}$ NMR $\left(126 \mathrm{MHz}, \mathrm{CDCl}_{3}\right) \delta 210.5,144.7,135.7,134.0,129.8,127.8,110.3,63.2$, 41.1, 39.3, 31.7, 27.0, 26.8, 22.8, 19.4. HRMS (ESI+): $\mathrm{m} / z$ calc'd for $\mathrm{C}_{25} \mathrm{H}_{35} \mathrm{O}_{2} \mathrm{Si}$ $[\mathrm{M}+\mathrm{H}]^{+}:$395.2401, found 395.2394.

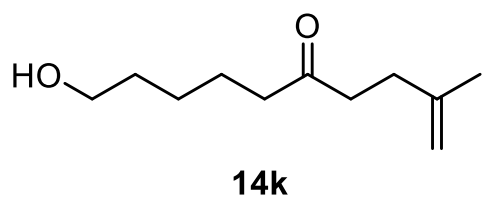

$14 k$

10-hydroxy-2-methyldec-1-en-5-one (14k): Obtained according to the general procedure $\mathrm{B}$ from cyclopropanol $11 \mathrm{~g}$ and 2-bromoprop-1-ene. The crude product was purified by flash column chromatography on silica gel (30\% EtOAc/PE) to provide a colorless oil (30.8 mg, $0.167 \mathrm{mmol}, 67 \%$ yield); ${ }^{1} \mathbf{H}$ NMR $\left(500 \mathrm{MHz}, \mathrm{CDCl}_{3}\right) \delta 4.72(\mathrm{~s}, 1 \mathrm{H}), 4.64(\mathrm{~s}, 1 \mathrm{H}), 3.64(\mathrm{t}, J=6.6 \mathrm{~Hz}, 2 \mathrm{H}), 2.56$ $-2.50(\mathrm{~m}, 2 \mathrm{H}), 2.43(\mathrm{t}, J=7.3 \mathrm{~Hz}, 2 \mathrm{H}), 2.29-2.23(\mathrm{~m}, 2 \mathrm{H}), 1.72(\mathrm{~s}, 3 \mathrm{H}), 1.65-$ $1.52(\mathrm{~m}, 4 \mathrm{H}), 1.51$ (br s, 1H), $1.40-1.30(\mathrm{~m}, 2 \mathrm{H}) .{ }^{13} \mathbf{C ~ N M R}\left(126 \mathrm{MHz}, \mathrm{CDCl}_{3}\right) \delta$ 210.7 , 144.7, 110.2, 62.8, 42.8, 41.1, 32.6, 31.6, 25.5, 23.5, 22.8. HRMS (ESI+): $m / z$ calc'd for $\mathrm{C}_{11} \mathrm{H}_{21} \mathrm{O}_{2}[\mathrm{M}+\mathrm{H}]^{+}:$185.1536, found 185.1533 .

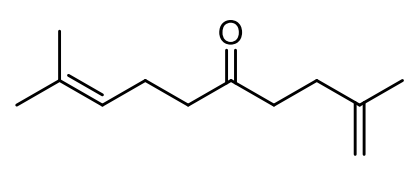

141

2,9-dimethyldeca-1,8-dien-5-one (14l): Obtained according to the general procedure B from cyclopropanol 11c and 2bromoprop-1-ene. The crude product was purified by flash column chromatography on silica gel (2\% EtOAc/PE) to provide a colorless oil $(29.3 \mathrm{mg}, 0.163 \mathrm{mmol}, 65 \%$ yield $) ;{ }^{1} \mathbf{H}$ NMR $(500 \mathrm{MHz}$, $\left.\mathrm{CDCl}_{3}\right) \delta 5.10-5.02(\mathrm{~m}, 1 \mathrm{H}), 4.72(\mathrm{~s}, 1 \mathrm{H}), 4.64(\mathrm{~s}, 1 \mathrm{H}), 2.57-2.50(\mathrm{~m}, 2 \mathrm{H}), 2.44$ $(\mathrm{t}, J=7.5 \mathrm{~Hz}, 2 \mathrm{H}), 2.31-2.21(\mathrm{~m}, 4 \mathrm{H}), 1.72(\mathrm{~s}, 3 \mathrm{H}), 1.67(\mathrm{~s}, 3 \mathrm{H}), 1.61(\mathrm{~s}, 3 \mathrm{H}) .{ }^{13} \mathrm{C}$ NMR $\left(126 \mathrm{MHz}, \mathrm{CDCl}_{3}\right) \delta 210.4,144.7,132.8,122.9,110.2,42.9,41.1,31.6,25.8$, 22.8, 22.7, 17.8. HRMS (ESI + ): $m / z$ calc'd for $\mathrm{C}_{12} \mathrm{H}_{21} \mathrm{O}[\mathrm{M}+\mathrm{H}]^{+}: 181.1587$, found 185.1587 . 


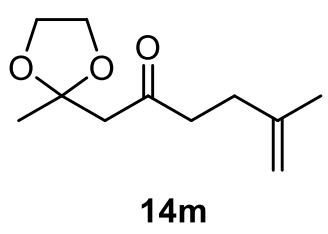

5-methyl-1-(2-methyl-1,3-dioxolan-2-yl)hex-5-en-2-one (14m): Obtained according to the general procedure B from cyclopropanol $\mathbf{1 1 h}$ and 2-bromoprop-1-ene. Upon reaction complete the crude mixture was poured into $5 \mathrm{~mL}$ saturated aqueous solution of $\mathrm{NH}_{4} \mathrm{Cl}$ and extracted with EtOAc $(3 \times 2 \mathrm{~mL})$.

The crude product was purified by flash column chromatography on silica gel $(5 \%$ EtOAc/PE) to provide a colorless oil (20.1 mg, $0.102 \mathrm{mmol}, 41 \%$ yield $) ;{ }^{1} \mathbf{H}$ NMR $\left(500 \mathrm{MHz}, \mathrm{CDCl}_{3}\right) \delta 4.71(\mathrm{~s}, 1 \mathrm{H}), 4.65(\mathrm{~s}, 1 \mathrm{H}), 4.00-3.91(\mathrm{~m}, 4 \mathrm{H}), 2.76(\mathrm{~s}, 2 \mathrm{H})$, $2.69-2.61(\mathrm{~m}, 2 \mathrm{H}), 2.26(\mathrm{t}, \mathrm{J}=7.6 \mathrm{~Hz}, 2 \mathrm{H}), 1.72(\mathrm{~s}, 3 \mathrm{H}), 1.40(\mathrm{~s}, 3 \mathrm{H}) .{ }^{13} \mathbf{C}$ NMR $\left(126 \mathrm{MHz}, \mathrm{CDCl}_{3}\right) \delta 207.2,144.7,110.2,108.1,64.7$ (2C), 51.8, 42.6, 31.3, 24.6, 22.8. HRMS (ESI+): $m / z$ calc'd for $\mathrm{C}_{11} \mathrm{H}_{18} \mathrm{NaO}_{3}[\mathrm{M}+\mathrm{Na}]^{+}:$221.1148, found 221.1141.

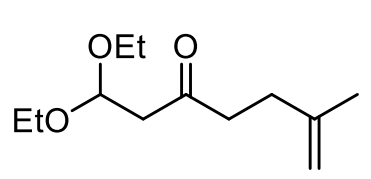

$14 n$

1,1-diethoxy-6-methylhept-6-en-3-one (14n): Obtained according to the general procedure $B$ from cyclopropanol 11d and 2-bromoprop-1-ene. Upon reaction complete the crude mixture was poured into $5 \mathrm{~mL}$ saturated aqueous solution of $\mathrm{NH}_{4} \mathrm{Cl}$ and extracted with EtOAc $(3 \times 2 \mathrm{~mL})$. The crude product was purified by flash column chromatography on silica gel $(3 \rightarrow 4 \%$ EtOAc/PE) to provide a colorless oil (34.8 mg, $0.162 \mathrm{mmol}, 65 \%$ yield); ${ }^{1} \mathbf{H}$ NMR (500 MHz, $\left.\mathrm{CDCl}_{3}\right) \delta 4.89$ $(\mathrm{t}, J=5.7 \mathrm{~Hz}, 1 \mathrm{H}), 4.71(\mathrm{~s}, 1 \mathrm{H}), 4.64(\mathrm{~s}, 1 \mathrm{H}), 3.66(\mathrm{dq}, J=9.3,7.1 \mathrm{~Hz}, 2 \mathrm{H}), 3.52$ (dq, $J=9.3,7.1 \mathrm{~Hz}, 2 \mathrm{H}), 2.73(\mathrm{~d}, J=5.7 \mathrm{~Hz}, 2 \mathrm{H}), 2.63-2.56(\mathrm{~m}, 2 \mathrm{H}), 2.27(\mathrm{~d}, J=$ $7.7 \mathrm{~Hz}, 2 \mathrm{H}), 1.71(\mathrm{~s}, 3 \mathrm{H}), 1.18(\mathrm{t}, J=7.1 \mathrm{~Hz}, 6 \mathrm{H}) .{ }^{13} \mathbf{C}$ NMR $\left(126 \mathrm{MHz}, \mathrm{CDCl}_{3}\right) \delta$ 207.4, 144.5, 110.2, 100.2, 47.7, 42.3, 31.1, 22.8, 15.4. HRMS (ESI+): $m / z$ calc'd for $\mathrm{C}_{12} \mathrm{H}_{22} \mathrm{NaO}_{3}[\mathrm{M}+\mathrm{Na}]^{+}: 237.1461$, found 237.1459.

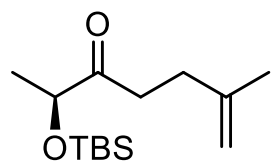

140

(S)-2-((tert-butyldimethylsilyl)oxy)-6-methylhept-6-en-3-one (140): Obtained according to the general procedure B from cyclopropanol 11i and 2-bromoprop-1-ene. The crude product was purified by flash column chromatography on silica gel $(2 \%$ EtOAc/PE) to provide a colorless oil $(30.8 \mathrm{mg}, 0.120 \mathrm{mmol}, 48 \%$ yield $) ;[\boldsymbol{\alpha}]_{\mathbf{D}^{20}}{ }^{\mathbf{2 0}}=-5.05\left(\mathrm{c}=0.55\right.$ in $\left.\mathrm{CHCl}_{3}\right){ }^{1} \mathbf{H} \mathbf{~ N M R}\left(500 \mathrm{MHz}, \mathrm{CDCl}_{3}\right) \delta 4.72(\mathrm{~s}, 1 \mathrm{H})$, 4.66 (s, 1H), 4.15 (q, $J=6.8 \mathrm{~Hz}, 1 \mathrm{H}), 2.82-2.65(\mathrm{~m}, 2 \mathrm{H}), 2.25(\mathrm{t}, J=7.7 \mathrm{~Hz}, 2 \mathrm{H})$, $1.73(\mathrm{~s}, 3 \mathrm{H}), 1.28(\mathrm{~d}, J=6.8 \mathrm{~Hz}, 3 \mathrm{H}), 0.92(\mathrm{~s}, 9 \mathrm{H}), 0.08(\mathrm{~s}, 6 \mathrm{H}) .{ }^{13} \mathbf{C}$ NMR $(126$ $\left.\mathrm{MHz}, \mathrm{CDCl}_{3}\right) \delta 213.8,144.9,110.0,75.0,35.3,31.0,25.9,22.9,21.0,18.2,-4.5$, 4.9. ee determined by the Mosher method was $93 \%$. Selected ${ }^{1} \mathrm{H}$ NMR chemical shifts for (S)-6-methyl-3-oxohept-6-en-2-yl (S)-3,3,3-trifluoro-2-methoxy-2phenylpropanoate: $\delta 5.28\left(\mathrm{q}, \mathrm{J}=7.0 \mathrm{~Hz}, 1 \mathrm{H}, \mathrm{CH}_{3} \mathrm{CHO}\right), 1.45(\mathrm{~d}, \mathrm{~J}=7.0 \mathrm{~Hz}, 3 \mathrm{H}$, $\mathrm{CH}_{3} \mathrm{CHO}$ ). Selected ${ }^{1} \mathrm{H}$ NMR chemical shifts for (S)-6-methyl-3-oxohept-6-en-2-yl 
(R)-3,3,3-trifluoro-2-methoxy-2-phenylpropanoate: $\delta 5.32(\mathrm{q}, \mathrm{J}=7.0 \mathrm{~Hz}, 1 \mathrm{H}$, $\left.\mathrm{CH}_{3} \mathrm{CHO}\right), 1.51\left(\mathrm{~d}, \mathrm{~J}=7.0 \mathrm{~Hz}, 3 \mathrm{H}, \mathrm{CH}_{3} \mathrm{CHO}\right.$ ). HRMS (ESI+): $\mathrm{m} / z$ calc'd for $\mathrm{C}_{14} \mathrm{H}_{28} \mathrm{NaO}_{2} \mathrm{Si}[\mathrm{M}+\mathrm{Na}]^{+}: 279.1751$, found 279.1754 .

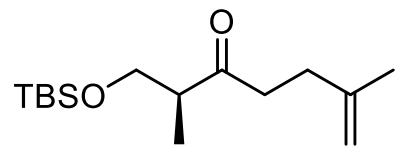

$14 p$

(S)-1-((tert-butyldimethylsilyl)oxy)-2,6-dimethylhept-6en-3-one (14p): Obtained according to the general procedure B from cyclopropanol 11j and 2-bromoprop-1-ene. The crude product was purified by flash column chromatography on silica gel (2\% EtOAc/PE) to provide a colorless oil $(37.2 \mathrm{mg}$, $0.138 \mathrm{mmol}, 55 \%$ yield $) ;[\boldsymbol{\alpha}]_{\mathbf{D}}{ }^{\mathbf{2 0}}=+29.28\left(\mathrm{c}=0.69\right.$ in $\left.\mathrm{CHCl}_{3}\right),{ }^{1} \mathbf{H} \mathbf{N M R}(500 \mathrm{MHz}$, $\left.\mathrm{CDCl}_{3}\right) \delta 4.71(\mathrm{~s}, 1 \mathrm{H}), 4.66(\mathrm{~s}, 1 \mathrm{H}), 3.72(\mathrm{dd}, J=9.8,7.7 \mathrm{~Hz}, 1 \mathrm{H}), 3.61(\mathrm{dd}, J=9.8$, $5.4 \mathrm{~Hz}, 1 \mathrm{H}), 2.83-2.74(\mathrm{~m}, 1 \mathrm{H}), 2.71-2.57(\mathrm{~m}, 2 \mathrm{H}), 2.26(\mathrm{t}, J=7.7 \mathrm{~Hz}, 2 \mathrm{H}), 1.73$ $(\mathrm{s}, 3 \mathrm{H}), 1.02(\mathrm{~d}, J=6.9 \mathrm{~Hz}, 3 \mathrm{H}), 0.86(\mathrm{~s}, 9 \mathrm{H}), 0.03(\mathrm{~s}, 3 \mathrm{H}), 0.02(\mathrm{~s}, 3 \mathrm{H}) .{ }^{13} \mathbf{C}$ NMR $\left(126 \mathrm{MHz}, \mathrm{CDCl}_{3}\right) \delta 213.3,144.9,110.1,65.8,48.7,41.2,31.2,26.0,22.8,18.3$, 13.2, -5.4. HRMS (ESI+): $m / z$ calc'd for $\mathrm{C}_{15} \mathrm{H}_{31} \mathrm{O}_{2} \mathrm{Si}[\mathrm{M}+\mathrm{H}]^{+}: 271.2088$, found 271.2086. 
Fragments of ${ }^{1} \mathrm{H}$ NMR spectra of (S)-6-methyl-3-oxohept-6-en-2-yl (R)-3,3,3trifluoro-2-methoxy-2-phenylpropanoate (up) and (S)-6-methyl-3-oxohept-6-en-2yl (S)-3,3,3-trifluoro-2-methoxy-2-phenylpropanoate (under):

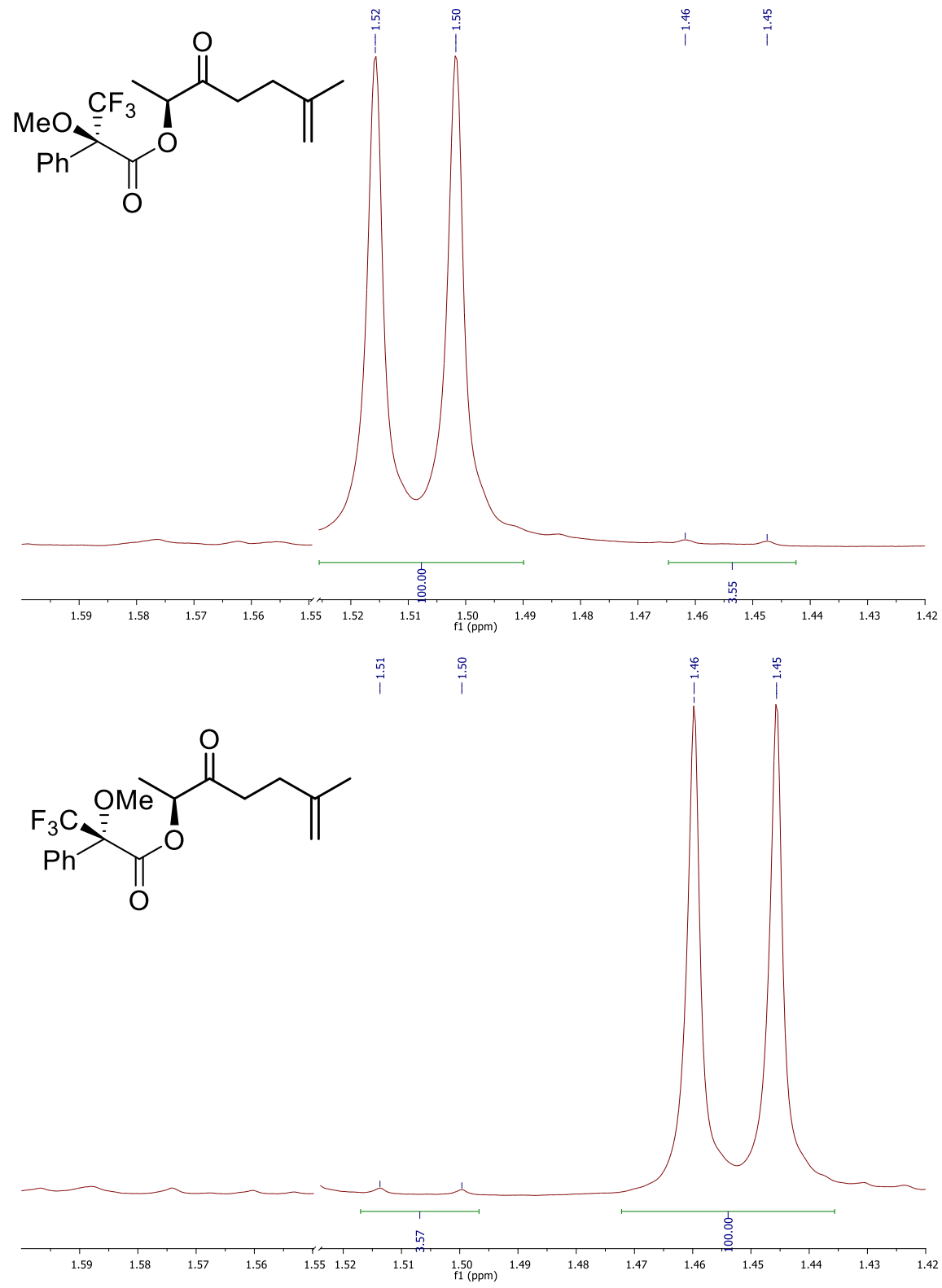


Retention of ee was observed for 14p.

Racemic product rac-14p: Astec CHIRALDEX B-DM, He at $12 \mathrm{psi}, 0.75 \mathrm{~mL} / \mathrm{min}$, $110{ }^{\circ} \mathrm{C}$, FID.

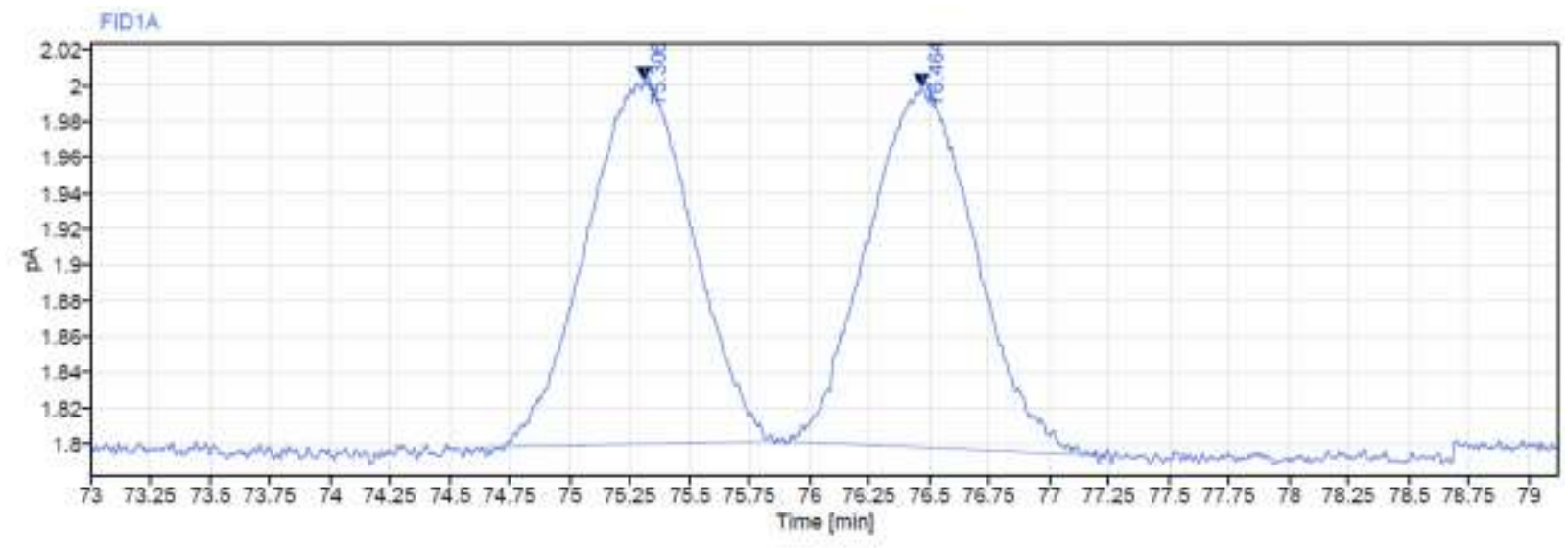

$\begin{array}{rrrrrr}\text { Signal: } & \text { FID1A } & & & & \\ \text { RT [min] } & \text { Type } & \text { Width [min] } & \text { Area } & \text { Height } & \text { Area\% } \\ 75.306 & \text { MM m } & 0.36 & 6.23 & 0.20 & 49.97 \\ 76.464 & \text { MM m } & 0.37 & 6.24 & 0.20 & 50.03 \\ & & \text { Sum } & 12.48 & & \end{array}$

Chiral product 14p: Astec CHIRALDEX B-DM, He at $12 \mathrm{psi}, 0.75 \mathrm{~mL} / \mathrm{min}, 110^{\circ} \mathrm{C}$, FID.

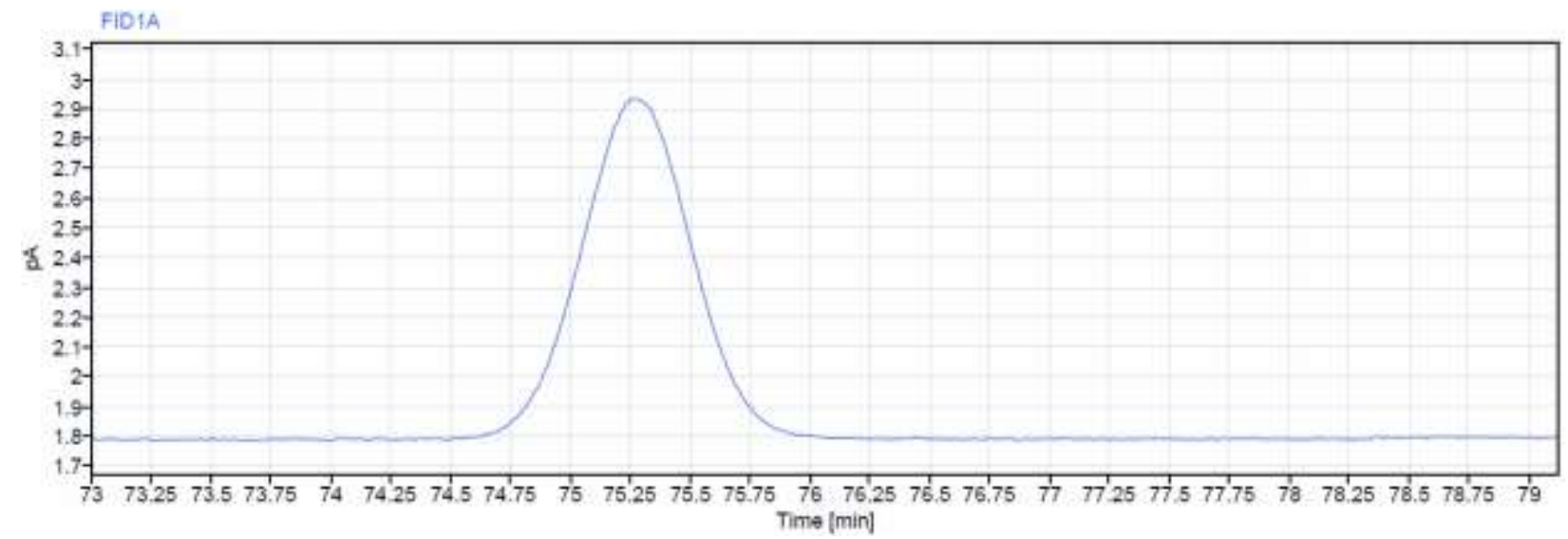

Racemic product rac-14p was obtained from corresponding chiral ketone according previously reported procedure with $\mathrm{Al}_{2} \mathrm{O}_{3}{ }^{10}$<smiles>C=C(C)C(C)CC(=O)CCc1ccccc1</smiles>

$14 q$

5,6-dimethyl-1-phenylhept-6-en-3-one (14q): Obtained according to the general procedure A from cyclopropanol 11e and 2-bromoprop-1-ene. The crude product was purified by flash column chromatography on silica gel $(2 \% \mathrm{EtOAc} / \mathrm{PE})$ to provide a colorless oil $(28.7 \mathrm{mg}, 0.133 \mathrm{mmol}, 53 \%$ yield $)$; ${ }^{1} \mathbf{H}$ NMR $\left(500 \mathrm{MHz}, \mathrm{CDCl}_{3}\right) \delta 7.31-7.26(\mathrm{~m}, 2 \mathrm{H}), 7.22-7.16(\mathrm{~m}, 3 \mathrm{H}), 4.71-4.68$ (m, 2H), 2.89 (t, J = 7.6 Hz, 2H), $2.75-2.66(\mathrm{~m}, 3 \mathrm{H}), 2.54$ (dd, J = 15.9, 6.4 Hz, 
1H), $2.34(\mathrm{dd}, \mathrm{J}=15.9,7.9 \mathrm{~Hz}, 1 \mathrm{H}), 1.70(\mathrm{~s}, 3 \mathrm{H}), 1.01(\mathrm{~d}, \mathrm{~J}=6.9 \mathrm{~Hz}, 3 \mathrm{H}) .{ }^{13} \mathbf{C}$ NMR $\left(126 \mathrm{MHz}, \mathrm{CDCl}_{3}\right) \delta 209.3,149.2,141.3,128.6(2 \mathrm{C}), 128.5(2 \mathrm{C}), 126.2,109.7,48.9$, 44.9, 36.7, 29.8, 20.2, 19.7. HRMS $(\mathrm{ESI}+): \mathrm{m} / \mathrm{z}$ calc'd for $\mathrm{C}_{15} \mathrm{H}_{21} \mathrm{O}[\mathrm{M}+\mathrm{H}]^{+}$: 217.1587, found 217.1587 .

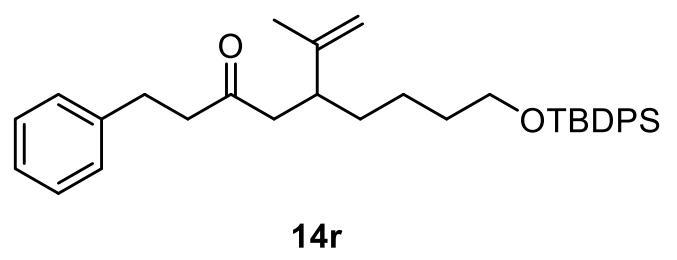

9-((tert-butyldiphenylsilyl)oxy)-1-phenyl-5(prop-1-en-2-yl)nonan-3-one (14r): Obtained according to the general procedure A from cyclopropanol $11 \mathrm{k}$ and 2-bromoprop-1-ene. The crude product was purified by flash column chromatography on silica gel ( $2 \%$ EtOAc/PE) to provide a colorless oil $(82.1 \mathrm{mg}, 0.160 \mathrm{mmol}, 64 \%$ yield $) ;{ }^{1} \mathbf{H}$ NMR $(500 \mathrm{MHz}$, $\left.\mathrm{CDCl}_{3}\right) \delta 7.69-7.64(\mathrm{~m}, 4 \mathrm{H}), 7.45-7.35(\mathrm{~m}, 6 \mathrm{H}), 7.30-7.25(\mathrm{~m}, 2 \mathrm{H}), 7.23-7.15$ $(\mathrm{m}, 3 \mathrm{H}), 4.74(\mathrm{~s}, 1 \mathrm{H}), 4.69(\mathrm{~s}, 1 \mathrm{H}), 3.64(\mathrm{t}, \mathrm{J}=6.4 \mathrm{~Hz}, 2 \mathrm{H}), 2.88(\mathrm{t}, \mathrm{J}=7.7 \mathrm{~Hz}, 2 \mathrm{H})$, $2.70(\mathrm{td}, \mathrm{J}=7.4,2.9 \mathrm{~Hz}, 2 \mathrm{H}), 2.65-2.55(\mathrm{~m}, 1 \mathrm{H}), 2.46(\mathrm{dd}, \mathrm{J}=15.5,7.9 \mathrm{~Hz}, 1 \mathrm{H})$, $2.37(\mathrm{dd}, \mathrm{J}=15.5,6.5 \mathrm{~Hz}, 1 \mathrm{H}), 1.62(\mathrm{~s}, 3 \mathrm{H}), 1.57-1.45(\mathrm{~m}, 2 \mathrm{H}), 1.31-1.20(\mathrm{~m}$, 4H), 1.05 (s, 9H). ${ }^{13}$ C NMR (126 MHz, $\left.\mathrm{CDCl}_{3}\right) \delta 209.3,146.7,141.3,135.7$ (4C), 134.3, $129.6(2 \mathrm{C}), 128.6(2 \mathrm{C}), 128.5$ (2C), 127.7 (4C), 126.2 (2C), 111.9, 63.9, 47.8, 44.8, 42.8, 33.1, 32.6, 29.7, 27.0 (3C), 23.6, 19.4, 19.0. HRMS (ESI+): $m / z$ calc'd for $\mathrm{C}_{34} \mathrm{H}_{45} \mathrm{O}_{2} \mathrm{Si}[\mathrm{M}+\mathrm{H}]^{+}:$513.3183, found 513.3177 .

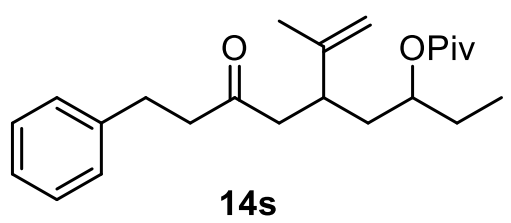

7-oxo-9-phenyl-5-(prop-1-en-2-yl)nonan-3-yl pivalate (14s): Obtained according to the general procedure A from cyclopropanol $( \pm)-\mathbf{1 1 1}$ and 2bromoprop-1-ene. The crude product was purified by flash column chromatography on silica gel $(3 \%$ EtOAc/PE) to provide 14s as a 1:1 mixture of diastereomers, colorless oil $(36.4 \mathrm{mg}$, $0.102 \mathrm{mmol}, 41 \%$ yield); ${ }^{1} \mathbf{H}$ NMR $\left(500 \mathrm{MHz}, \mathrm{CDCl}_{3}\right) \delta 7.30-7.24(\mathrm{~m}, 2 \mathrm{H}), 7.21$ $-7.14(\mathrm{~m}, 3 \mathrm{H}), 4.79-4.77(\mathrm{~m}, 0.5 \mathrm{H}), 4.75-4.72(\mathrm{~m}, 0.5 \mathrm{H}), 4.71(\mathrm{~s}, 0.5 \mathrm{H}), 4.67(\mathrm{~s}$, $0.5 \mathrm{H}), 2.90-2.83(\mathrm{~m}, 2 \mathrm{H}), 2.77-2.61(\mathrm{~m}, 3 \mathrm{H}), 2.50(\mathrm{dd}, \mathrm{J}=15.8,7.4 \mathrm{~Hz}, 0.5 \mathrm{H})$, $2.47-2.44(\mathrm{~m}, 1 \mathrm{H}), 2.39(\mathrm{dd}, \mathrm{J}=15.8,7.0 \mathrm{~Hz}, 0.5 \mathrm{H}), 1.70(\mathrm{~s}, 1.5 \mathrm{H}), 1.68-1.64$ $(\mathrm{m}, 1 \mathrm{H}), 1.62(\mathrm{~s}, 1.5 \mathrm{H}), 1.58-1.41(\mathrm{~m}, 3 \mathrm{H}), 1.21(\mathrm{~s}, 4 \mathrm{H}), 1.19(\mathrm{~s}, 5 \mathrm{H}), 1.18-1.14$ $(\mathrm{m}, 1 \mathrm{H}), 0.88(\mathrm{t}, \mathrm{J}=7.4 \mathrm{~Hz}, 1.5 \mathrm{H}), 0.84(\mathrm{t}, \mathrm{J}=7.4 \mathrm{~Hz}, 1.5 \mathrm{H}) .{ }^{13} \mathbf{C} \mathbf{N M R}(126 \mathrm{MHz}$, $\left.\mathrm{CDCl}_{3}\right) \delta 208.8,208.7,178.4,178.2,146.3,145.8,141.2,128.6(2 \mathrm{C}), 128.4(2 \mathrm{C})$, 126.2, 126.2, 112.9, 112.1, 73.1, 72.8, 47.9, 47.2, 44.7, 39.4, 39.1, 39.0, 37.2, 37.0, 29.8, 29.7, 29.7, 27.5, 27.4 (3C), 27.3 (3C), 26.6, 19.2, 19.1, 9.4, 9.4. HRMS (ESI+): $m / z$ calc'd for $\mathrm{C}_{23} \mathrm{H}_{34} \mathrm{NaO}_{3}[\mathrm{M}+\mathrm{Na}]^{+}: 381.2400$, found 381.2402 . 
<smiles>C=C(C)C1CCc2cc(OC)ccc2C(=O)C1</smiles>

2-methoxy-7-(prop-1-en-2-yl)-6,7,8,9-tetrahydro-5Hbenzo[7]annulen-5-one (14t): Obtained according to the general procedure A from cyclopropanol $11 \mathrm{~m}$ and 2bromoprop-1-ene. The crude product was purified by flash column chromatography on silica gel (3\% EtOAc/PE) to provide a colorless oil $(33.0 \mathrm{mg}, 0.143 \mathrm{mmol}, 57 \%$ yield $) ;{ }^{1} \mathbf{H}$ NMR $(500 \mathrm{MHz}$, $\left.\mathrm{CDCl}_{3}\right) \delta 8.01(\mathrm{~d}, \mathrm{~J}=8.7 \mathrm{~Hz}, 1 \mathrm{H}), 6.82(\mathrm{dd}, \mathrm{J}=8.7,2.5 \mathrm{~Hz}, 1 \mathrm{H}), 6.68(\mathrm{~d}, \mathrm{~J}=2.5 \mathrm{~Hz}$, $1 \mathrm{H}), 4.83(\mathrm{~s}, 1 \mathrm{H}), 4.74(\mathrm{~s}, 1 \mathrm{H}), 3.85(\mathrm{~s}, 3 \mathrm{H}), 3.01-2.86(\mathrm{~m}, 2 \mathrm{H}), 2.79(\mathrm{dd}, \mathrm{J}=14.1$, $3.8 \mathrm{~Hz}, 1 \mathrm{H}), 2.63-2.54(\mathrm{~m}, 1 \mathrm{H}), 2.22-2.15(\mathrm{~m}, 1 \mathrm{H}), 2.11(\mathrm{dd}, \mathrm{J}=14.1,10.1 \mathrm{~Hz}$, 1H), $1.80-1.70(\mathrm{~m}, 4 \mathrm{H}) .{ }^{13} \mathbf{C}$ NMR $\left(126 \mathrm{MHz}, \mathrm{CDCl}_{3}\right) \delta 198.8,163.6,146.6,143.6$, 130.1, 126.3, 113.2, 112.6 (2C), 55.5, 45.0, 38.0, 28.8, 27.7, 22.1. HRMS (ESI+): $m / z$ calc'd for $\mathrm{C}_{15} \mathrm{H}_{19} \mathrm{O}_{2}[\mathrm{M}+\mathrm{H}]^{+}: 231.1380$, found 231.1378.

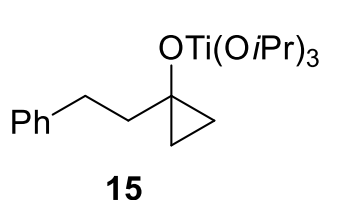

triisopropoxy(1-phenethylcyclopropoxy)titanium (15):

Prepared by stirring cyclopropanol 11a (1 eq.) and $\mathrm{Ti}(\mathrm{O} i \mathrm{Pr})_{4}$ (1 eq.) at room temperature under reduced pressure for 1 hour.

${ }^{1} \mathbf{H}$ NMR $\left(500 \mathrm{MHz}, \mathrm{CDCl}_{3}\right) \delta 7.30-7.14(\mathrm{~m}, 5 \mathrm{H}), 4.59-4.45$ $(\mathrm{m}, 3 \mathrm{H}), 2.97-2.89(\mathrm{~m}, 2 \mathrm{H}), 1.94-1.86(\mathrm{~m}, 2 \mathrm{H}), 1.27(\mathrm{~d}, J=6.1 \mathrm{~Hz}, 18 \mathrm{H}), 0.96-$ $0.83(\mathrm{~m}, 2 \mathrm{H}), 0.58-0.45(\mathrm{~m}, 2 \mathrm{H}) .{ }^{13} \mathrm{C}$ NMR $\left(126 \mathrm{MHz}, \mathrm{CDCl}_{3}\right) \delta 143.0,128.6$, $128.4,125.7,41.4,32.9,26.7,25.5,15.6$. 


\section{Mechanistic investigation}

\section{General procedure: w/o photocatalyst}

A $1.5 \mathrm{~mL}$ vial equipped with a stirring bar was charged with cyclopropanol 11a (20 $\mathrm{mg}, 0.12 \mathrm{mmol}, 1$ eq.), $\mathrm{NiCl}_{2} \cdot \mathrm{DME}(1.4 \mathrm{mg}, 6.25 \mu \mathrm{mol}, 2.5 \mathrm{~mol} \%$ ), 2,9-neocuproine (1.3 mg, $6.25 \mu \mathrm{mol}, 2.5 \mathrm{~mol} \%), \mathrm{K}_{2} \mathrm{CO}_{3}\left(51.2 \mathrm{mg}, 0.37 \mathrm{mmol}, 3\right.$ eq.) and $\mathrm{Ti}(\mathrm{O} i \mathrm{Pr})_{4}$ (73 $\mu \mathrm{L}, 0.25 \mathrm{mmol}, 2$ eq., for entry 1,3). The vial was purged with Ar for $5 \mathrm{~min}$ followed by addition of 2-bromoprop-1-ene ( $21 \mu \mathrm{L}, 0.25 \mathrm{mmol}, 2$ eq.) solution in acetone $(1.2 \mathrm{~mL})$. The resulting mixture was set to stir $(1200 \mathrm{rpm})$ and irradiated with light source ( $5 \mathrm{~cm}$ away) for $22 \mathrm{~h}$. Upon reaction complete the crude mixture was poured into $5 \% \mathrm{H}_{2} \mathrm{SO}_{4}$ solution ( $2 \mathrm{~mL}$; for entry 1 and 3$)$ or $\mathrm{H}_{2} \mathrm{O}(2 \mathrm{~mL}$; for entry 2 and 4$)$ and extracted with EtOAc $(3 \times 2 \mathrm{~mL})$. The combined organic layers were washed with water ( $2 \mathrm{~mL}$; for entry 1 and 3$)$, saturated solution of $\mathrm{NaHCO}_{3}$ ( $2 \mathrm{~mL}$; for entry 1 and 3), dried over $\mathrm{Na}_{2} \mathrm{SO}_{4}$ and concentrated under reduced pressure. Crude NMR with internal standard (dibromomethane) was recorded.

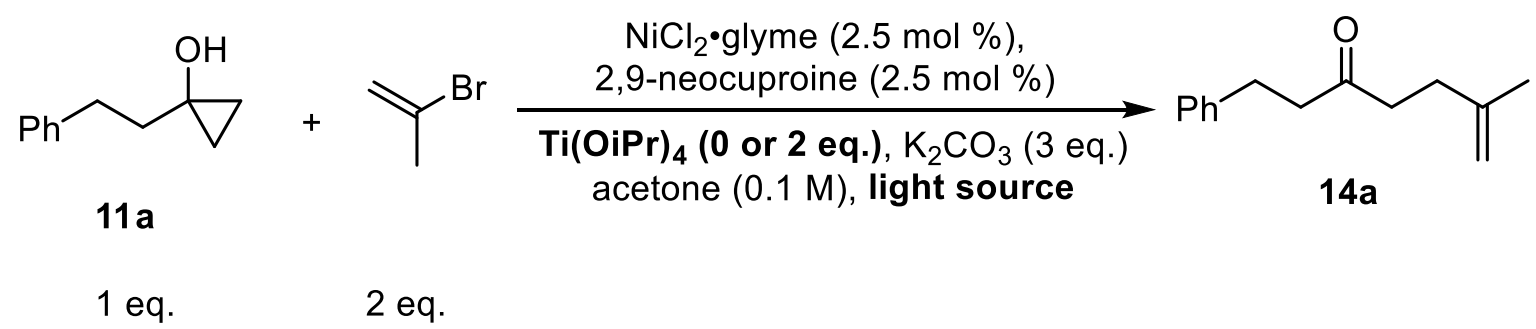

\begin{tabular}{cccc}
\hline Entry & Light source & Ti(OiPr) $)_{4}$, equiv. & NMR Yield of 14a, \% \\
\hline 1 & blue LEDs & 2 & 10 \\
2 & blue LEDs & - & - \\
3 & purple LEDs & 2 & 38 \\
4 & purple LEDs & - & 1 \\
\hline
\end{tabular}




\section{Cyclic Voltammetry}

The blank curve (Figure S1) indicates no considerable oxidation current, whereas addition of cyclopropanol 11a leads to appearance of anodic peak around $1.7 \mathrm{~V}$ vs $\mathrm{Fc}^{+} / \mathrm{Fc}$ which is attributed to cyclopropanol oxidation. A further addition of 1 equivalent of $\mathrm{Ti}(\mathrm{O} i \mathrm{Pr})_{4}$ does not change shape of the voltammogram noticeably, only slight current increase is observed

Thus, addition of $\mathrm{Ti}(\mathrm{O} i \mathrm{Pr})_{4}$ to reaction mixture does not change oxidation potential of 11a and appear not to affect oxidative ability of initial state of the compound.

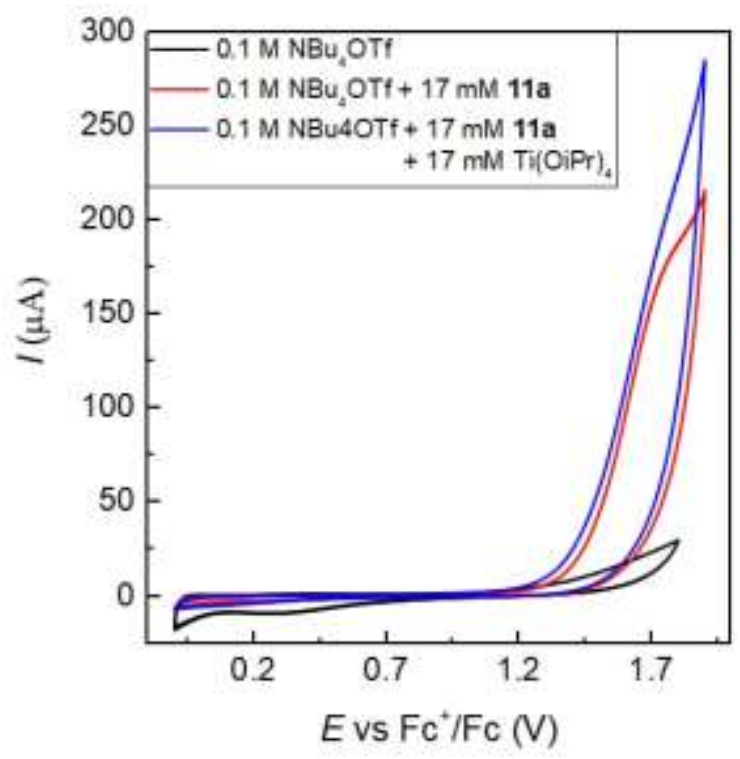

Figure S1. Cyclic voltammograms of Pt wire electrode in blank electrolyte (black curve), electrolyte with cyclopropanol 11a (red curve), electrolyte with cyclopropanol 11a and 1 equivalent of $\mathrm{Ti}(\mathrm{O} i \mathrm{Pr})_{4}$ (blue curve). Potential scan rate $0.1 \mathrm{~V} / \mathrm{s}$ 


\section{IPA/Cyclopropanol ligand exchange experiment}

To a solution of cyclopropanol 11a $(10 \mathrm{mg}, 0.062 \mathrm{mmol})$ in acetone- $\mathrm{d}^{6}(0.7 \mathrm{~mL})$ $\mathrm{Ti}(\mathrm{O} i \mathrm{Pr})_{4}(0.018 \mathrm{~mL}, 0.062 \mathrm{mmol})$ was added and ${ }^{1} \mathrm{H}$ NMR was recorded after 10 min at room temperature. Formation of 1:3 mixture of 11a and 15 was observed.

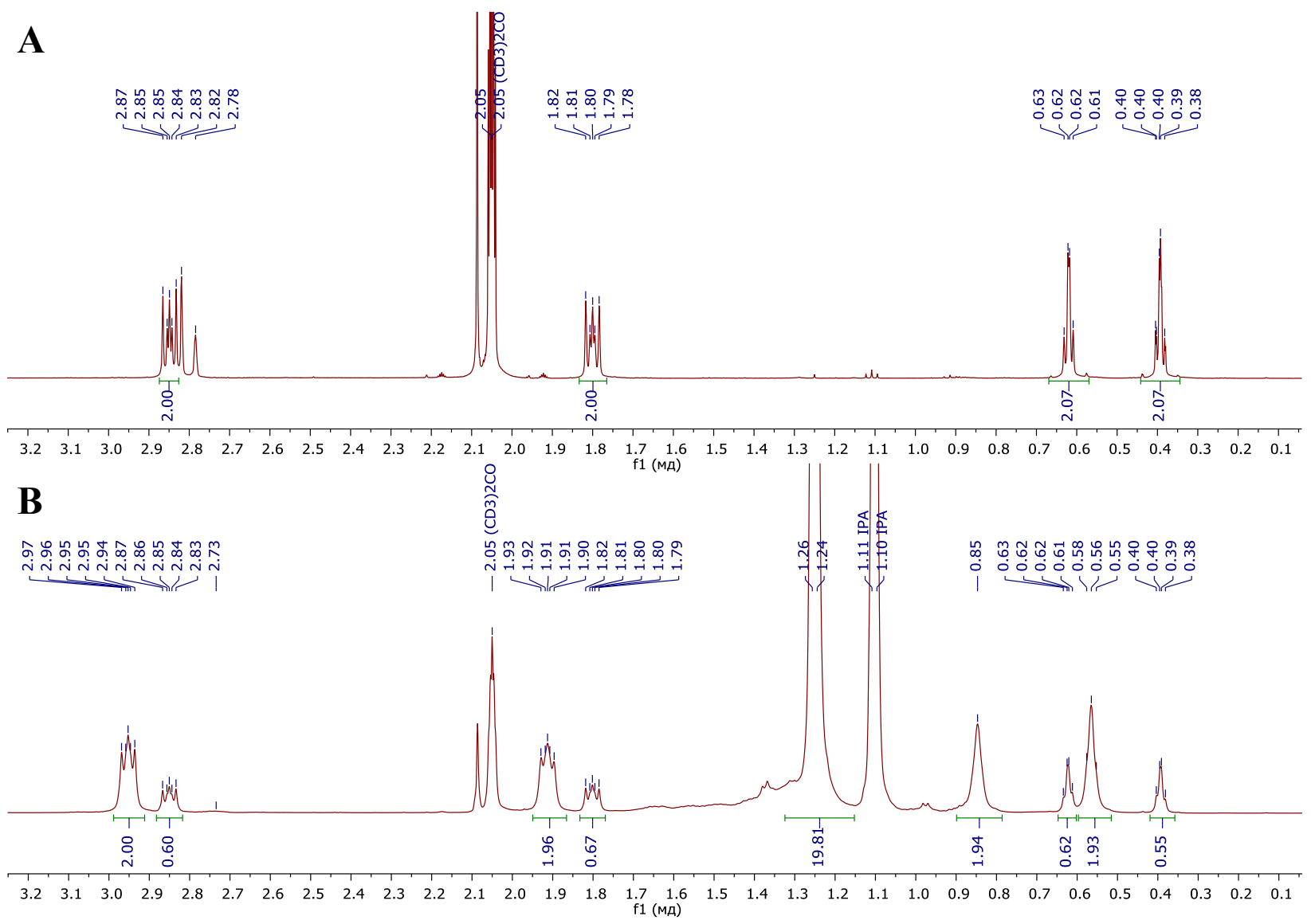

Figure S2. ${ }^{1} \mathrm{H}$ NMR $\left(500 \mathrm{MHz}\right.$, acetone- $\left.\mathrm{d}^{6}\right)$ of compound 11a (A) and 1:1 mixture of 11a and $\mathrm{Ti}(\mathrm{O} i \mathrm{Pr})_{4}$ after $10 \mathrm{~min}$ at room temperature (B). 


\section{References}

1. S. M. Engle, T. R. Kirkner, and C. B. Kelly, Org. Synth. 2019, 96, 455.

2. J. Das, M. Vellakkaran, and D. Banerjee, J. Org. Chem. 2019, 84, 769.

3. M.V. Laktsevich-Iskryk, N.A. Varabyeva, V.V. Kazlova, V.N. Zhabinskii, V.A. Khripach and A.L. Hurski, Eur. J. Org. Chem. 2020, 2431-2434.

4. M. Limbach, S. Dalai, A. de Meijere, Adv. Synth. Catal. 2004, 346, 760.

5. O. G. Kulinkovich, V. V. Bagutskii, Zh. Org. Khim. 1997, 33, 898-901; Russ. J. Org. Chem. (Engl. Transl.) 1997, 33, 830- 834.

6. M.V. Barysevich, M.V. Laktsevich-Iskryk, A.V. Krech, V.N. Zhabinskii, V.A. Khripach and A.L. Hurski, Eur. J. Org. Chem. $2020,937$.

7. D. G. Kananovich, Y. A. Konik, D. M. Zubrytski, I. Järving and M. Lopp, Chem. Commun. 2015, 51, 8349.

8. A. L. Hurski, Y. V. Ermolovich, V. N. Zhabinskii and V. A. Khripach, Org. Biomol. Chem. 2015, 13, 1446.

9. M. V. Laktsevich-Iskryk, A. V. Krech, V.N. Zhabinskii, V.A. Khripach and A. L. Hurski, Synth. 2021, 53, 1077-1086.

10. M. V. Barysevich, V. V Kazlova, A. G. Kukel, A. I. Liubina, A. L. Hurski, V. N. Zhabinskii, V. A. Khripach, Chem. Commun. 2018, 54, 2800. 


\section{6. ${ }^{1} \mathrm{H}$ NMR and ${ }^{13} \mathrm{C}$ NMR Spectra}

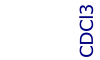

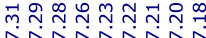

ind<smiles>OC1(CCc2ccccc2)CC1</smiles>

$11 \mathrm{a}$

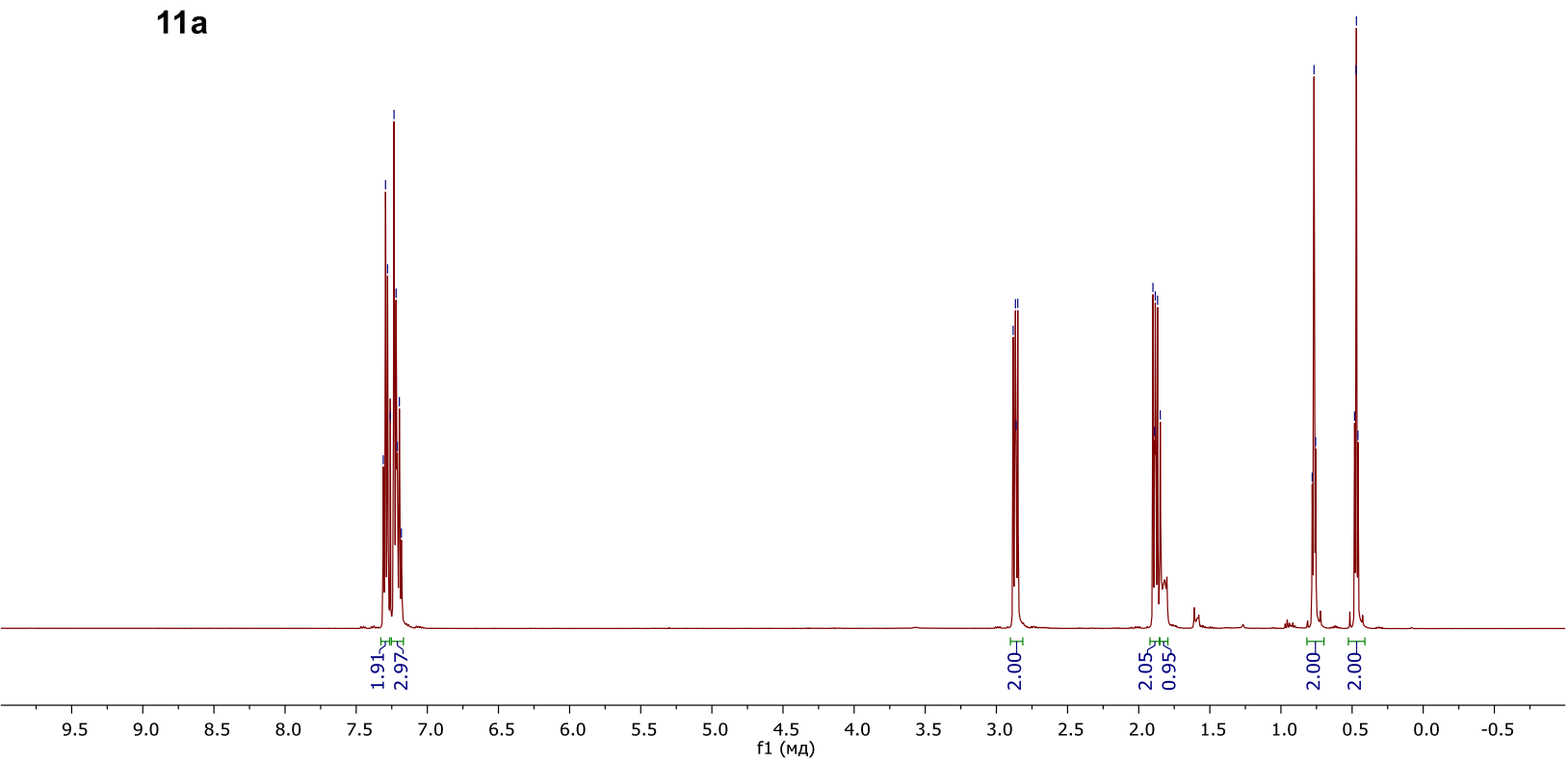

${ }^{1} \mathrm{H}$ NMR (500 MHz, $\left.\mathrm{CDCl}_{3}\right)$ of compound 11a

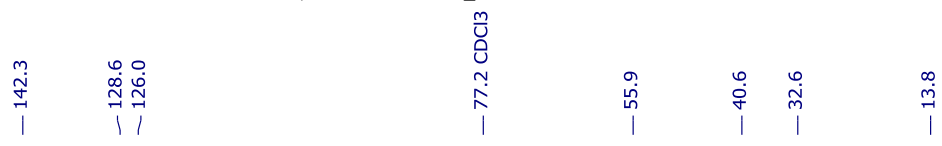<smiles>OC1(CCc2ccccc2)CC1</smiles>

11a

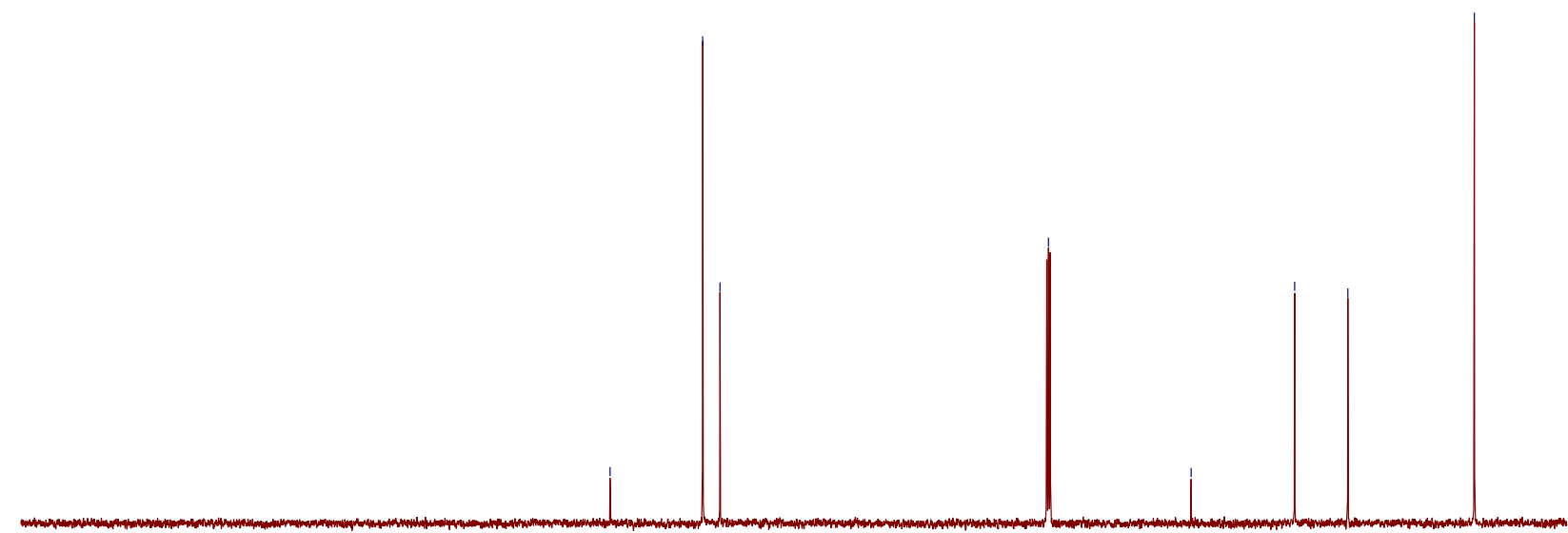

$\begin{array}{lllll}30 & 220 & 210 & 200 & 190\end{array}$

${ }^{13} \mathrm{C}$ NMR (126 MHz, $\left.\mathrm{CDCl}_{3}\right)$ of compound 11a 


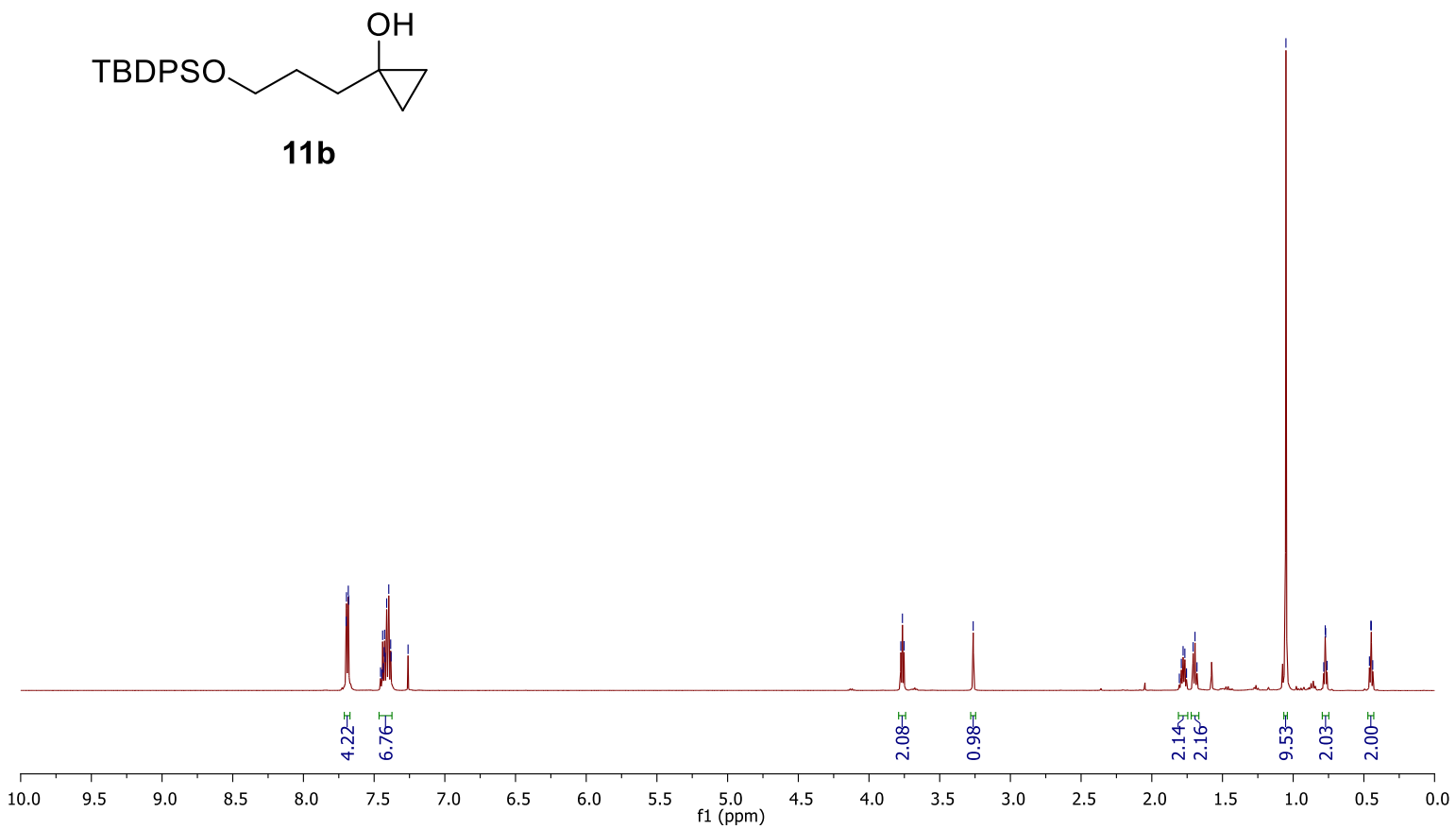

11b

${ }^{1} \mathrm{H}$ NMR (500 MHz, $\mathrm{CDCl}_{3}$ ) of compound $\mathbf{1 1 b}$

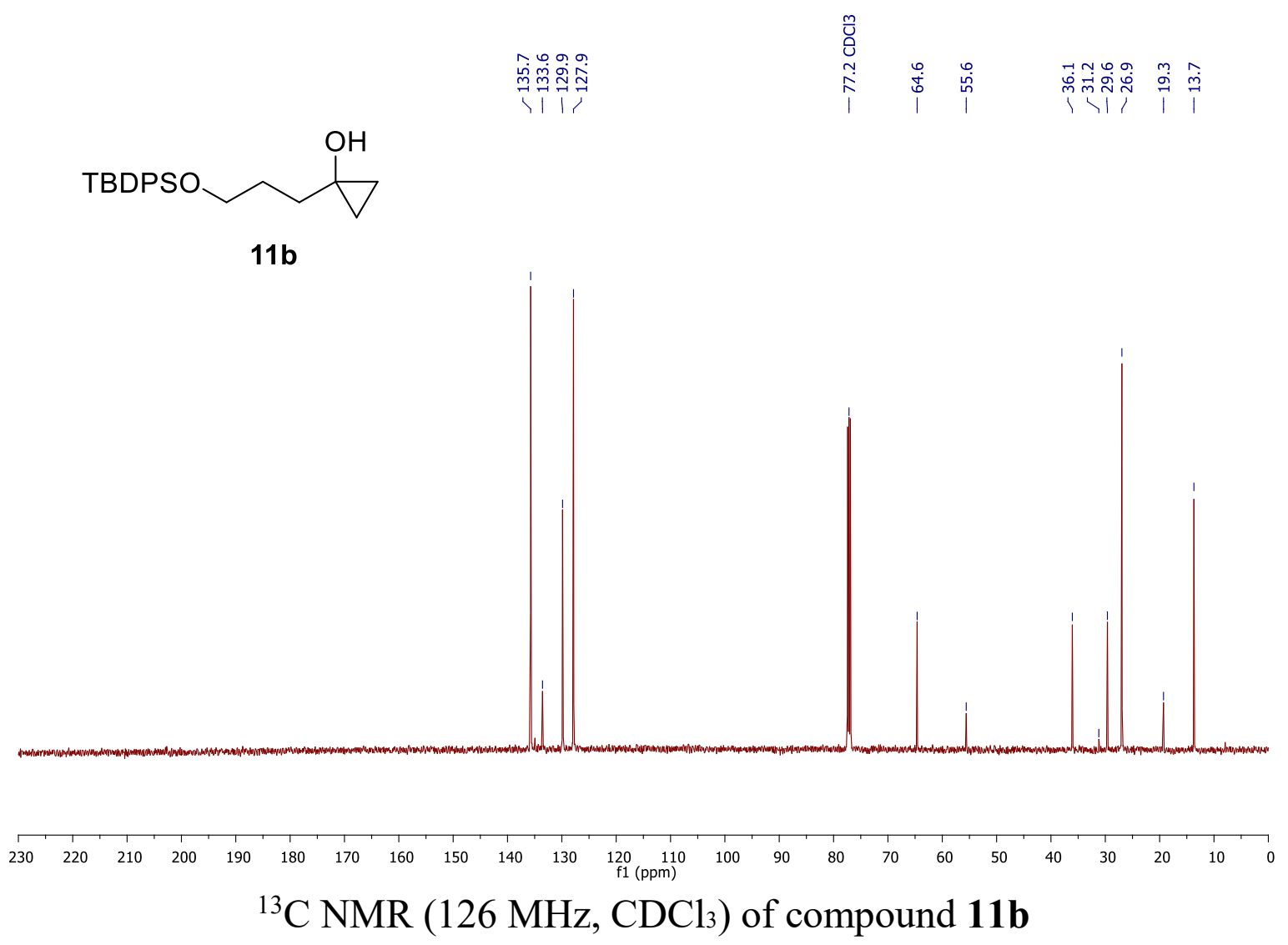



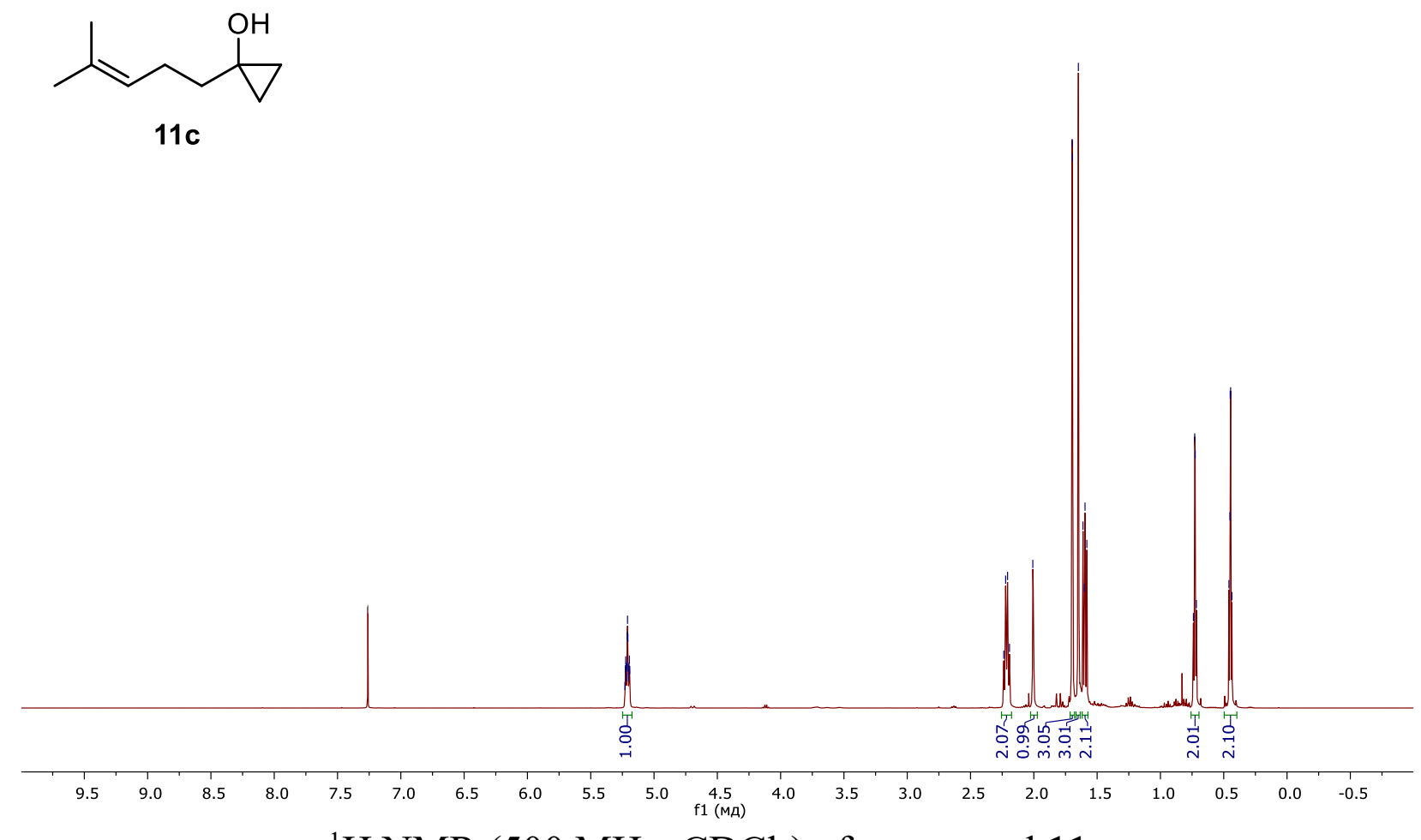

${ }^{1} \mathrm{H} \mathrm{NMR}\left(500 \mathrm{MHz}, \mathrm{CDCl}_{3}\right)$ of compound 11c<smiles>CC(C)=CCCC1(O)CC1</smiles>

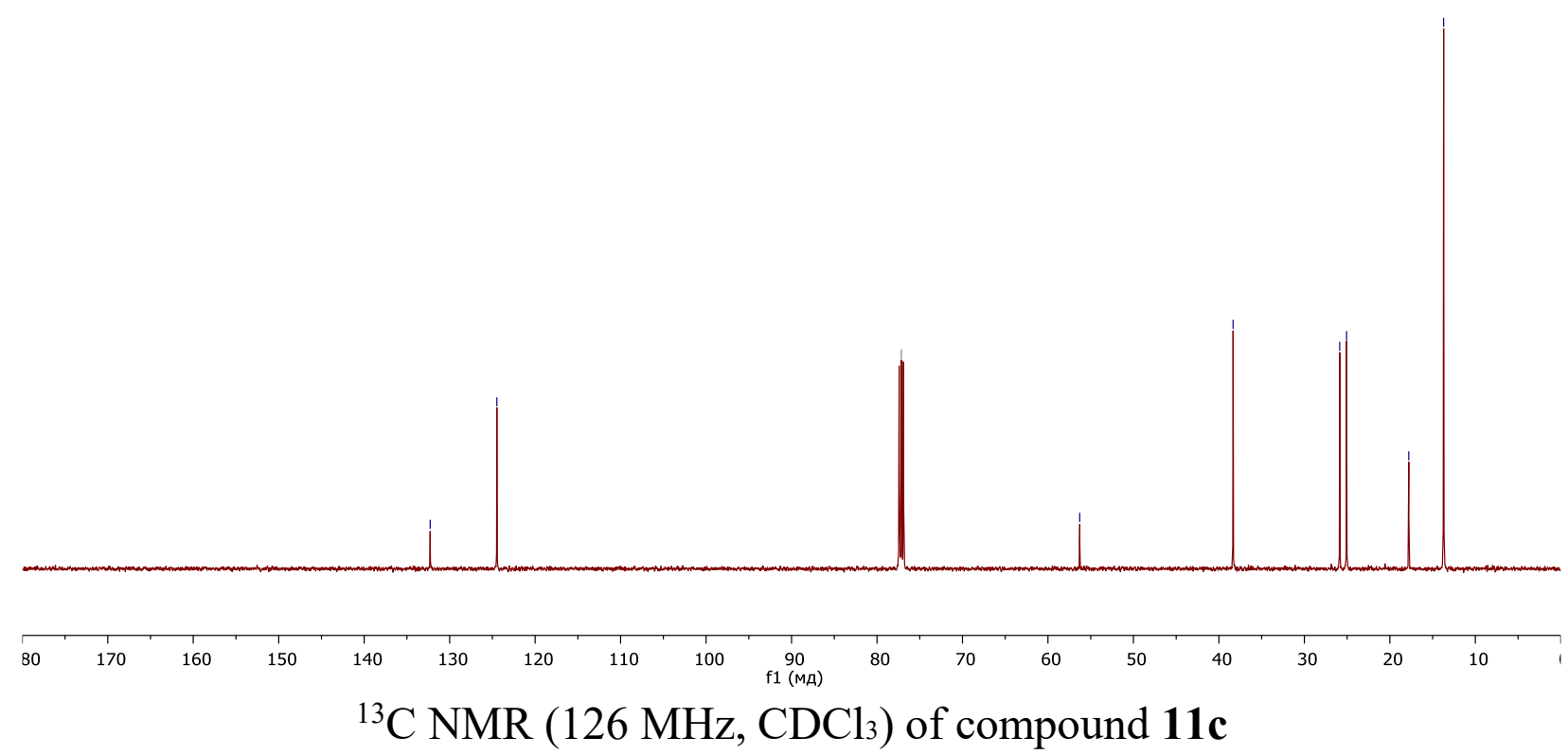


<smiles>CCOC(CC1(O)CC1)OCC</smiles>

11d

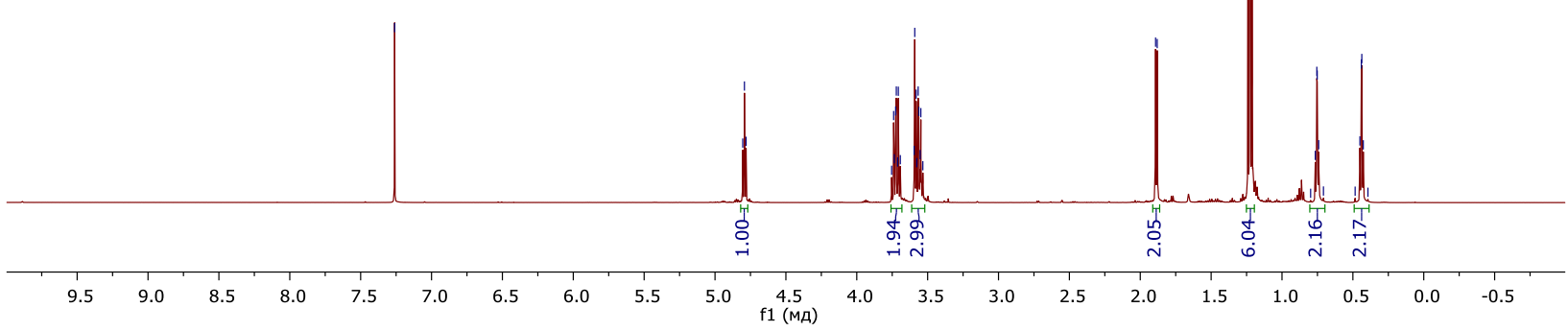

${ }^{1} \mathrm{H}$ NMR $\left(500 \mathrm{MHz}, \mathrm{CDCl}_{3}\right)$ of compound 11d

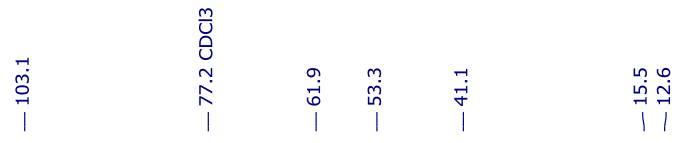<smiles>CCOC(CC1(O)CC1)OCC</smiles>

11d

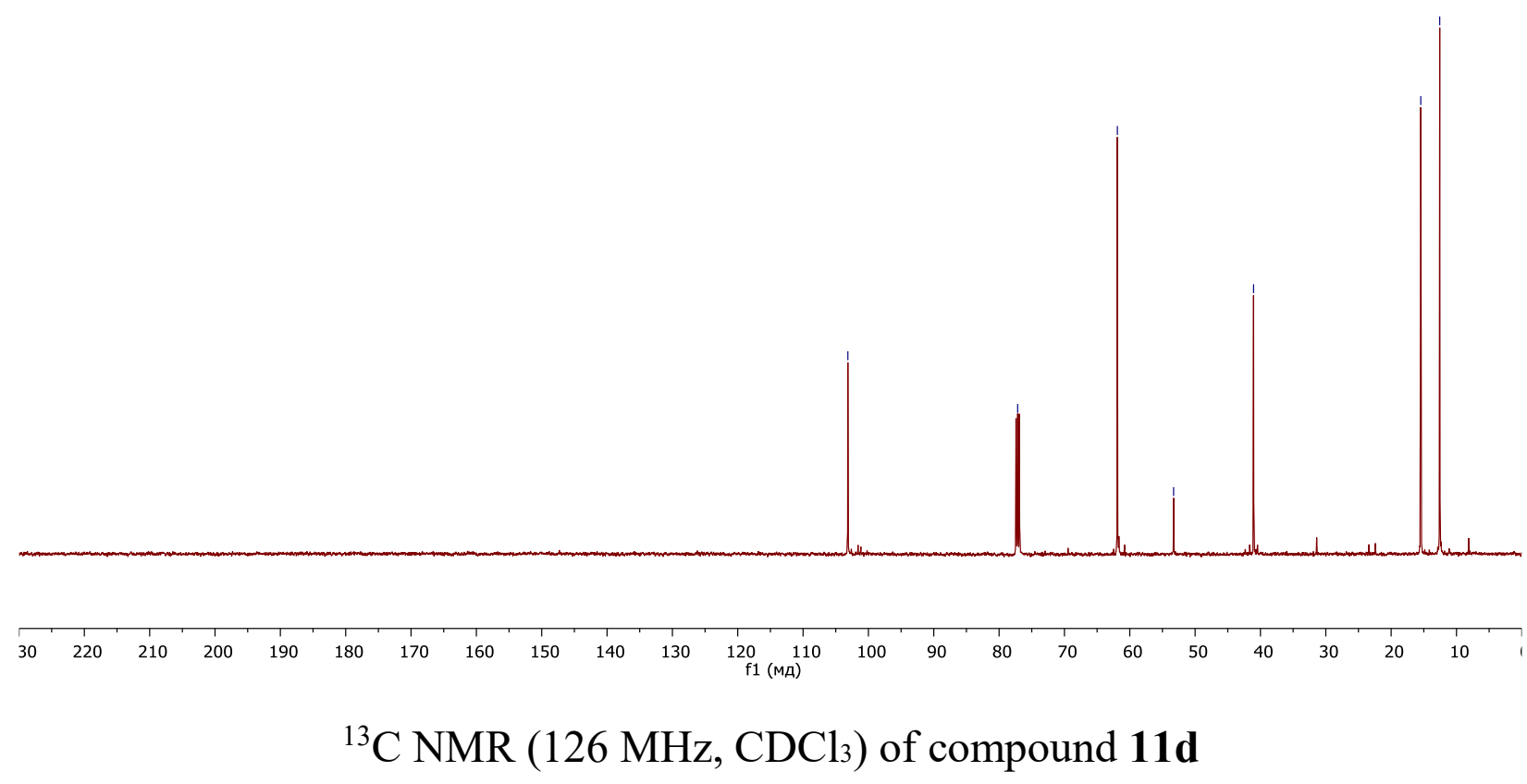


<smiles>CC1CC1(O)CCc1ccccc1</smiles>

$11 \mathrm{e}$

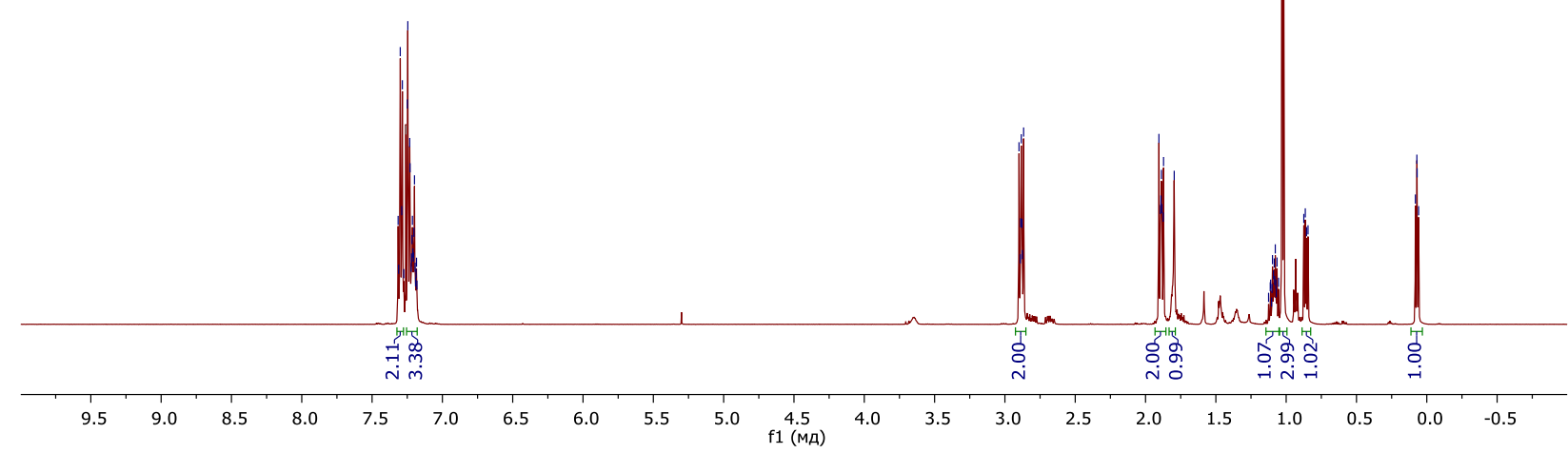

${ }^{1} \mathrm{H}$ NMR $\left(500 \mathrm{MHz}, \mathrm{CDCl}_{3}\right)$ of compound 11e<smiles>CC1CC1(O)CCc1ccccc1</smiles>

$11 \mathrm{e}$

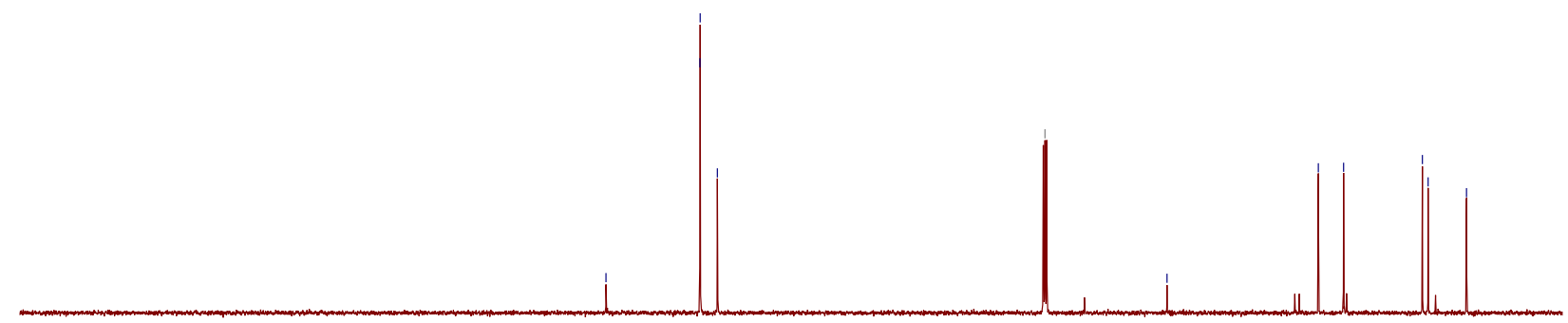

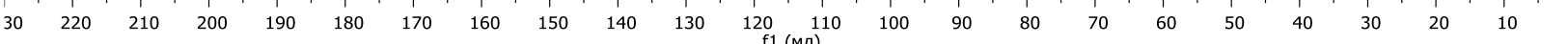

${ }^{13} \mathrm{C} \mathrm{NMR}\left(126 \mathrm{MHz}, \mathrm{CDCl}_{3}\right)$ of compound 11e 
<smiles>OC1(CCCCC2(O)CC2)CC1</smiles>

$11 \mathrm{f}$

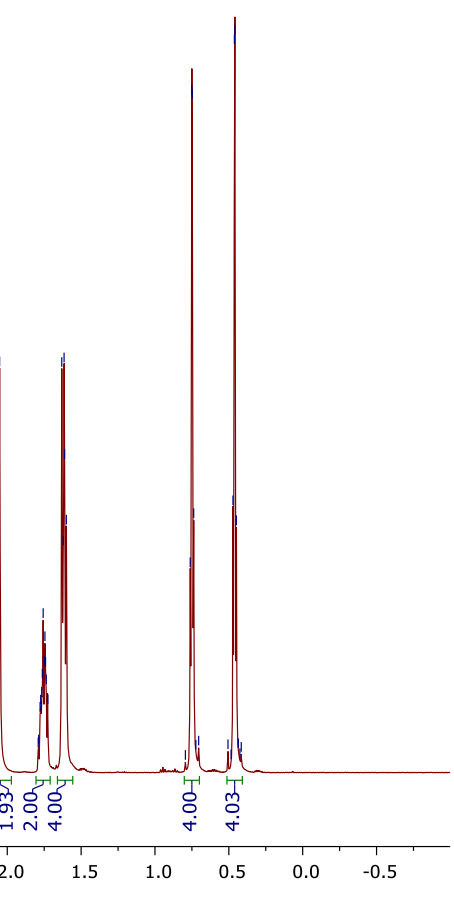

${ }^{1} \mathrm{H}$ NMR $\left(500 \mathrm{MHz}, \mathrm{CDCl}_{3}\right)$ of compound $11 \mathrm{f}$

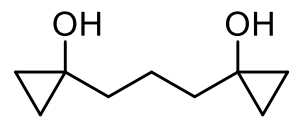

$11 f$

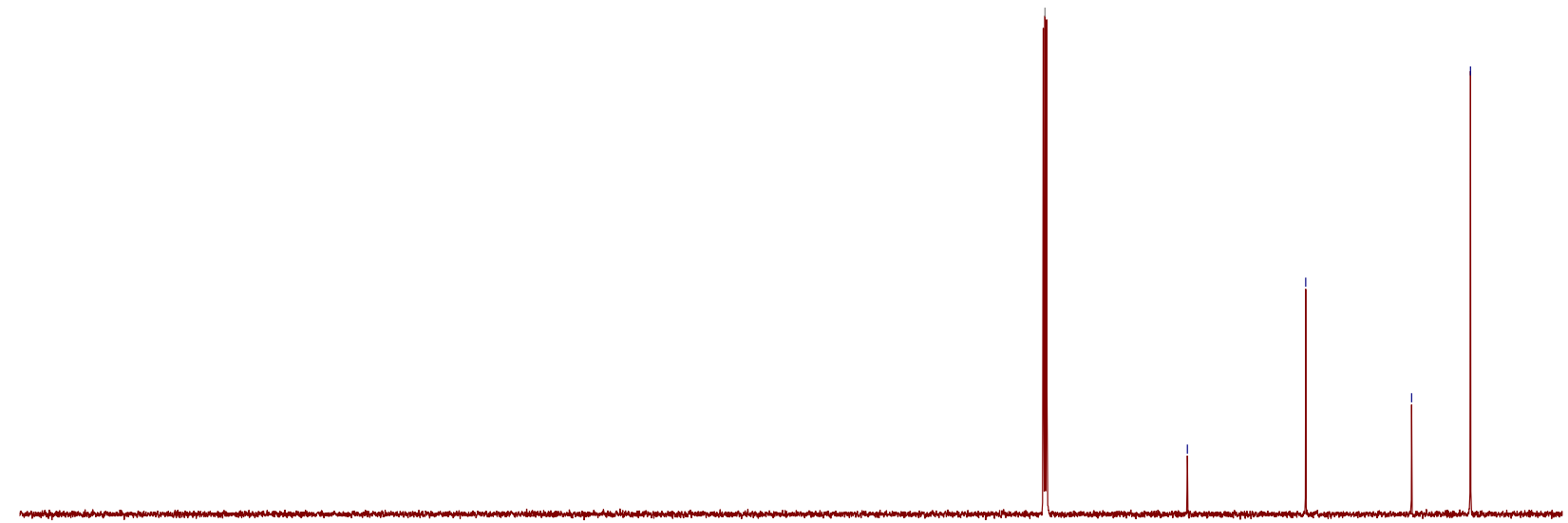

$\begin{array}{lllllllllllllllllllllll} & 220 & 210 & 200 & 190 & 180 & 170 & 160 & 150 & 140 & 130 & 120 & 110 & 100 & 90 & 80 & 70 & 60 & 50 & 40 & 30 & 20 & 10\end{array}$ ${ }^{13} \mathrm{C}$ NMR (126 MHz, $\mathrm{CDCl}_{3}$ ) of compound $11 \mathbf{f}$ 
<smiles>OCCCCCC1(O)CC1</smiles>

$11 \mathrm{~g}$

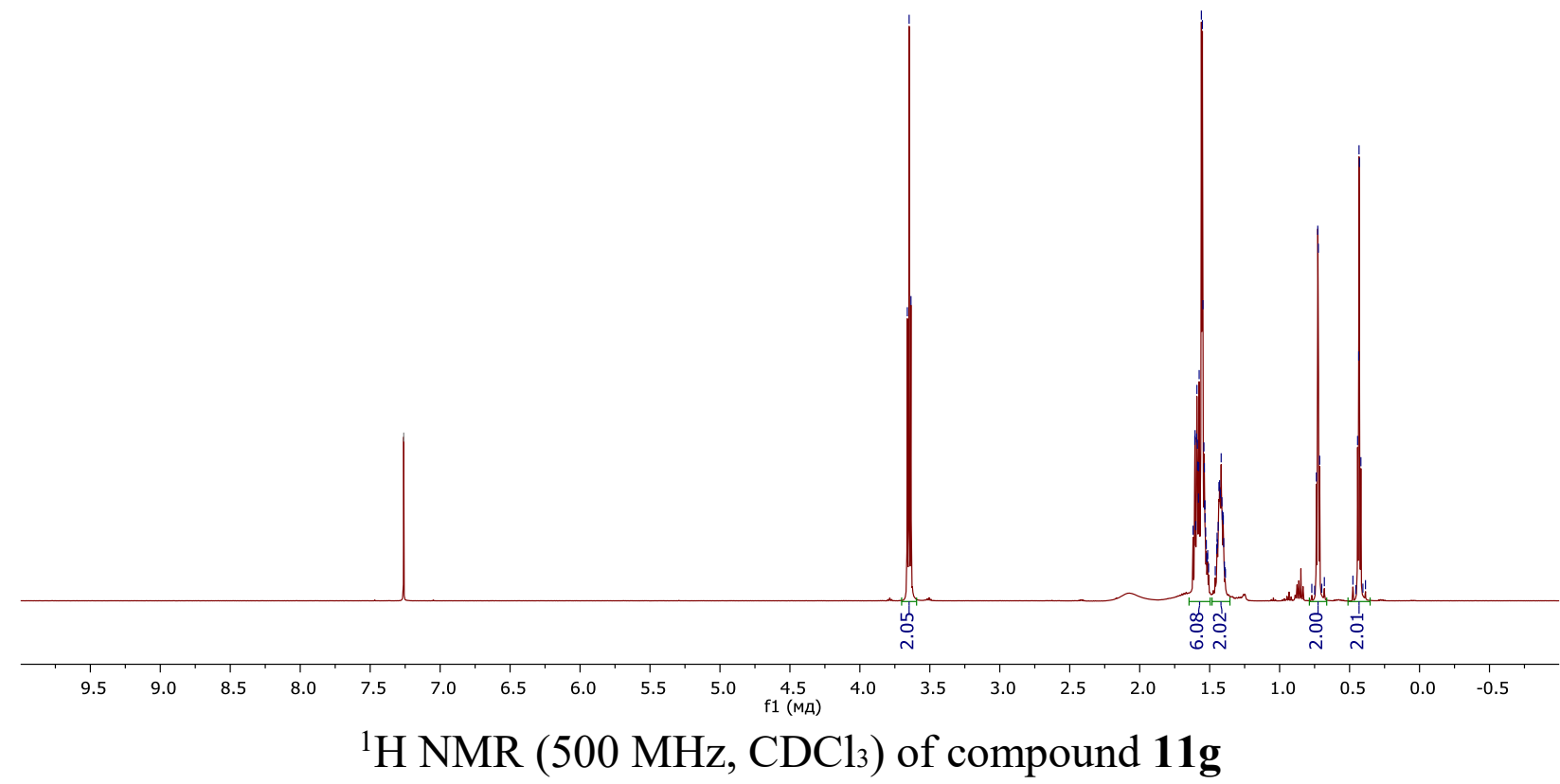

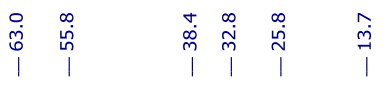<smiles>OCCCCCC1(O)CC1</smiles>

$11 \mathrm{~g}$

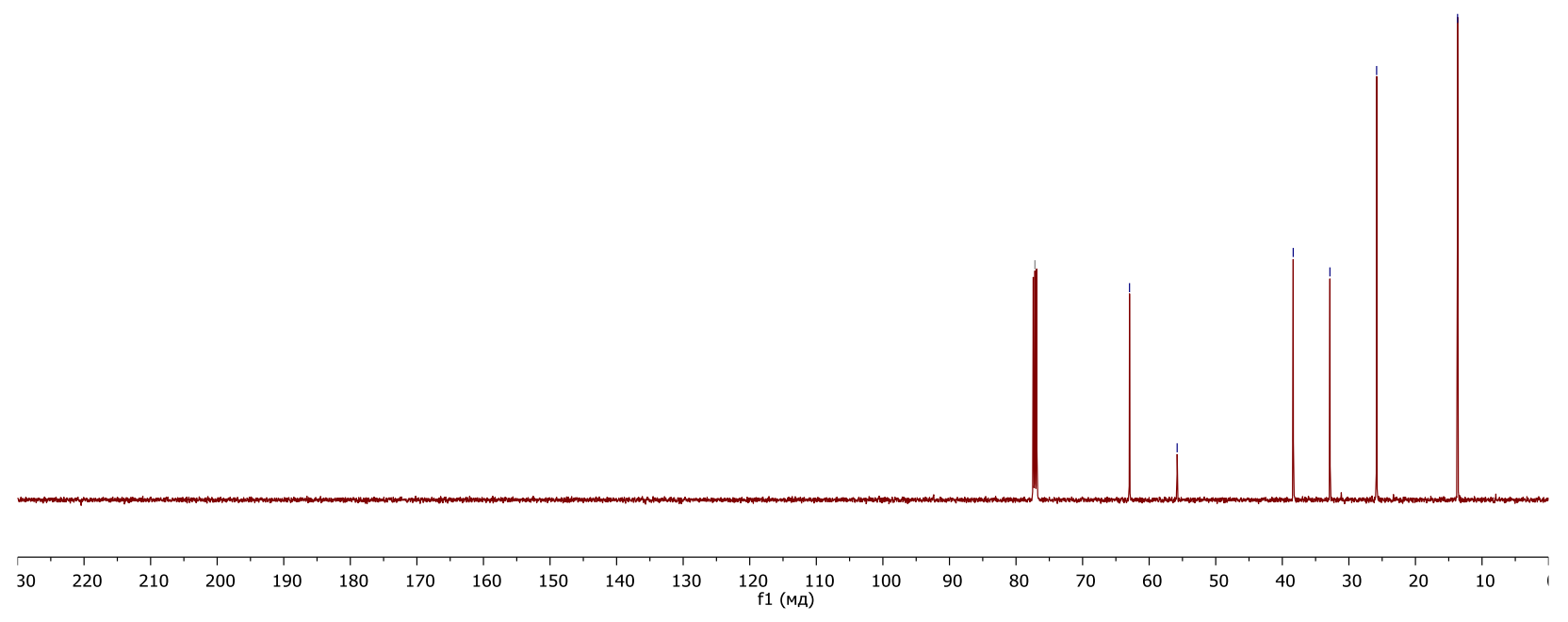

${ }^{13} \mathrm{C}$ NMR $\left(126 \mathrm{MHz}, \mathrm{CDCl}_{3}\right)$ of compound $\mathbf{1 1 g}$ 


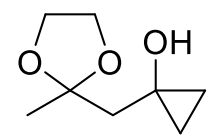

$11 \mathrm{~h}$

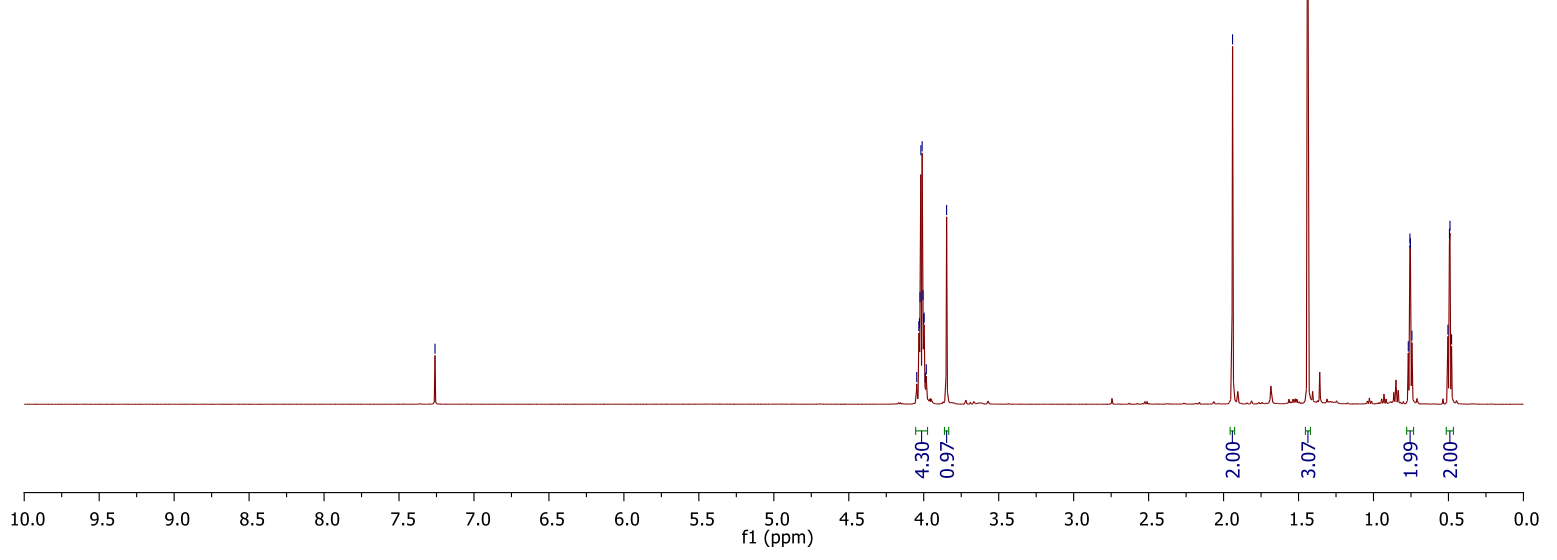

${ }^{1} \mathrm{H}$ NMR (500 MHz, $\left.\mathrm{CDCl}_{3}\right)$ of compound $\mathbf{1 1 h}$
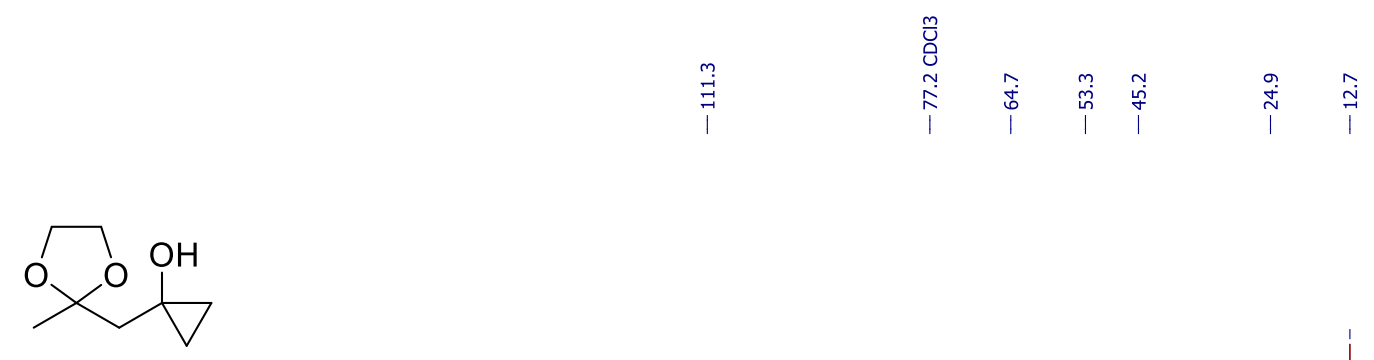

$11 \mathrm{~h}$

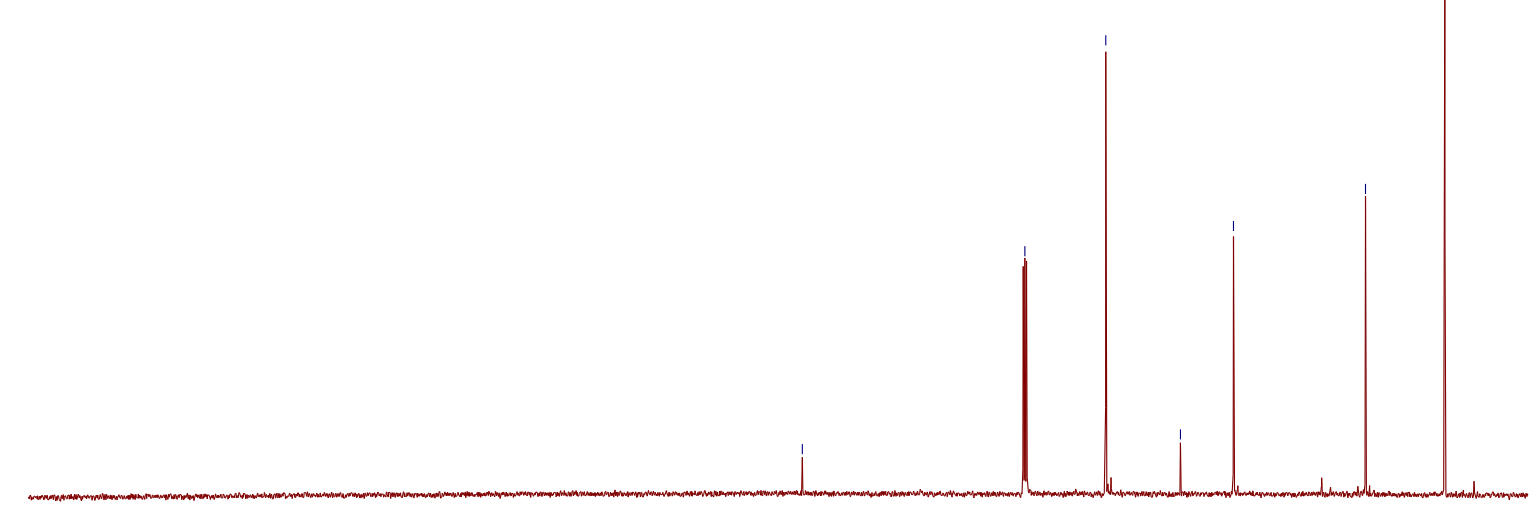

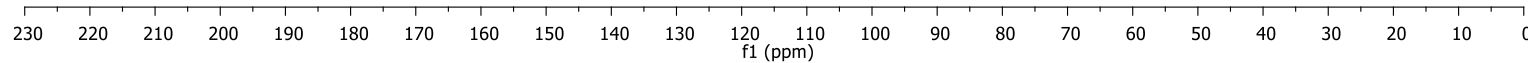

${ }^{13} \mathrm{C}$ NMR (126 MHz, $\left.\mathrm{CDCl}_{3}\right)$ of compound $\mathbf{1 1 h}$ 


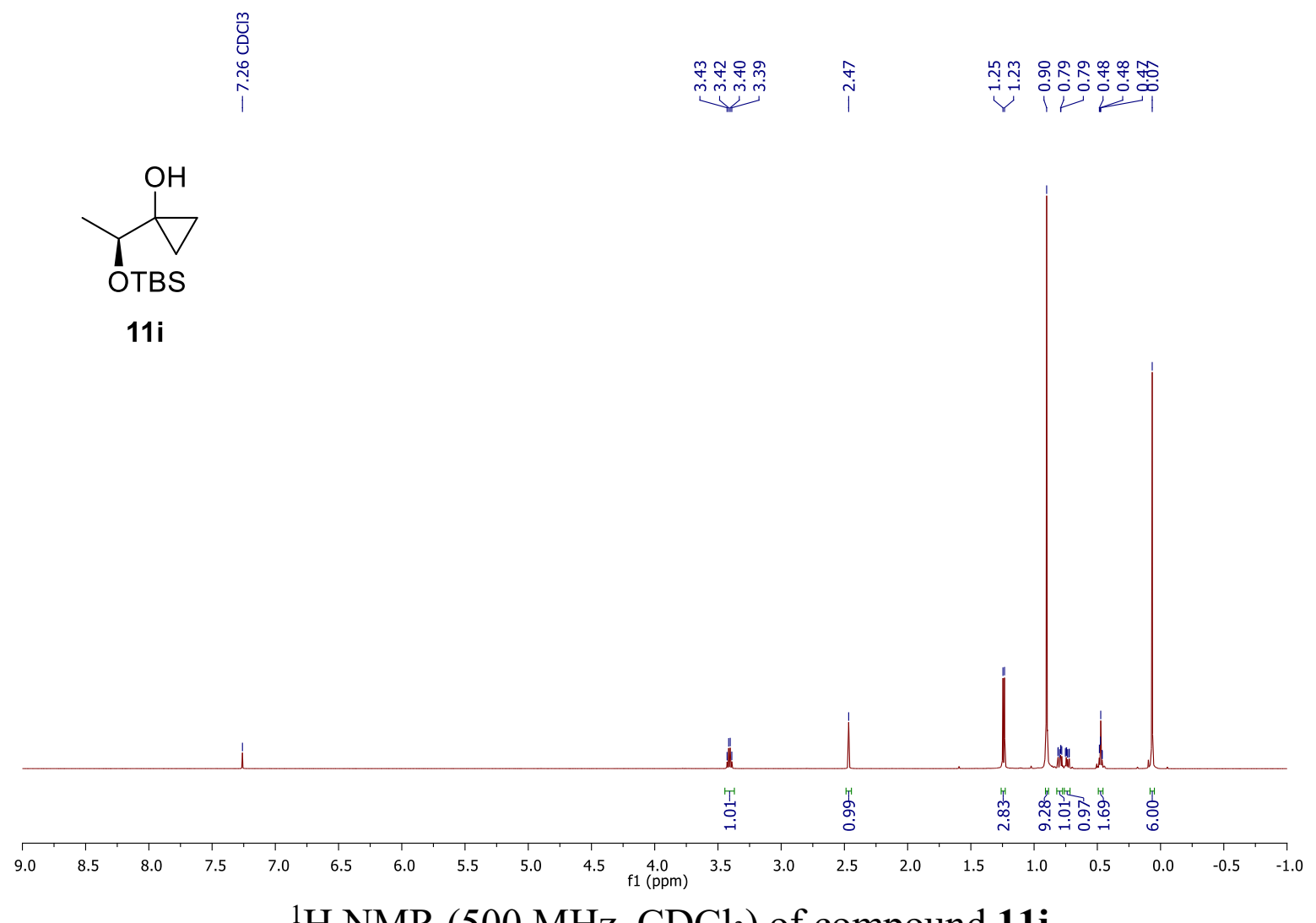

${ }^{1} \mathrm{H} \mathrm{NMR}\left(500 \mathrm{MHz}, \mathrm{CDCl}_{3}\right)$ of compound $\mathbf{1 1 i}$

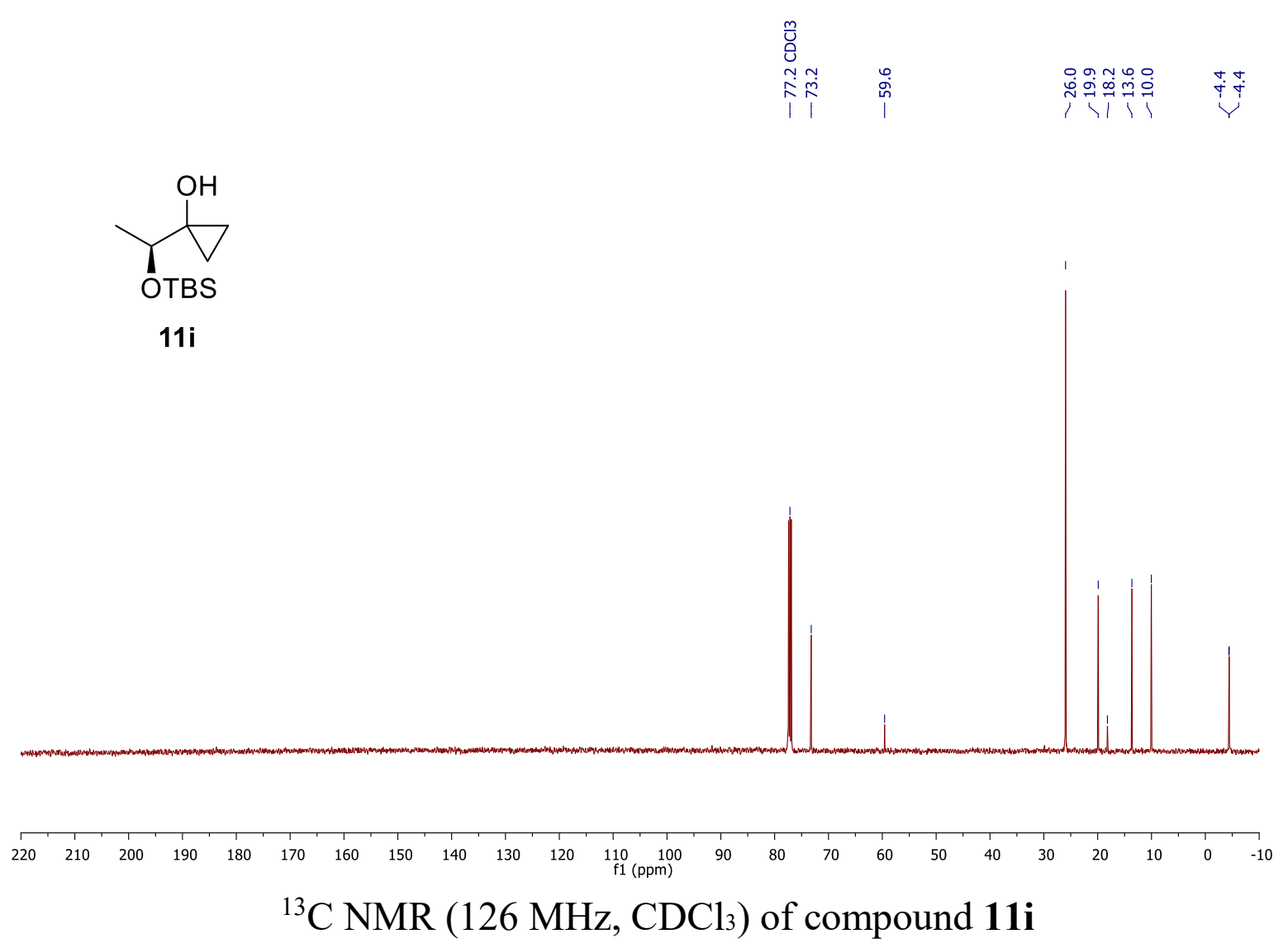




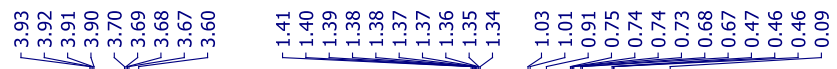

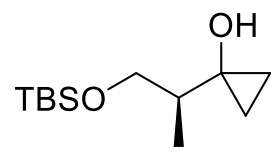

$11 \mathrm{j}$

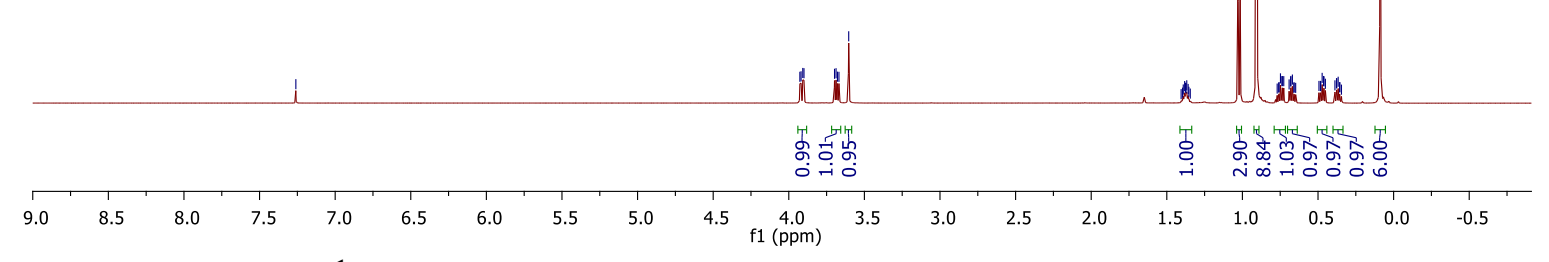

${ }^{1} \mathrm{H}$ NMR (500 MHz, $\mathrm{CDCl}_{3}$ ) of compound $\mathbf{1 1} \mathbf{j}$
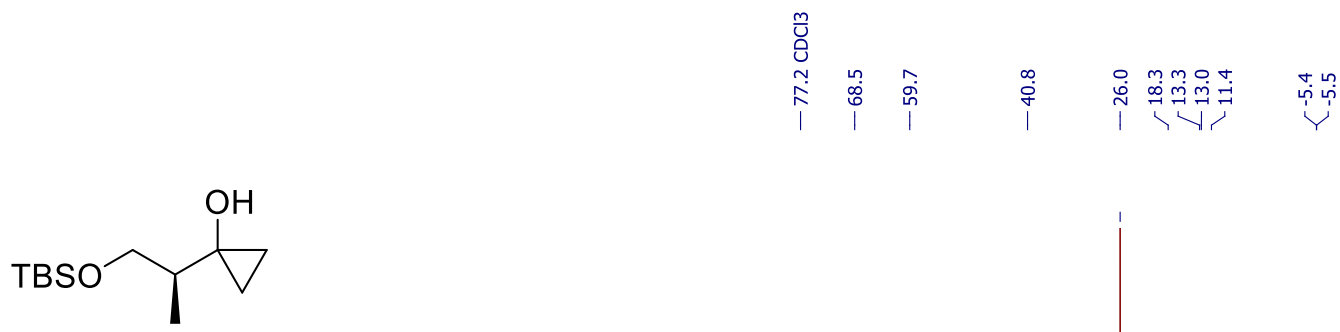

11j
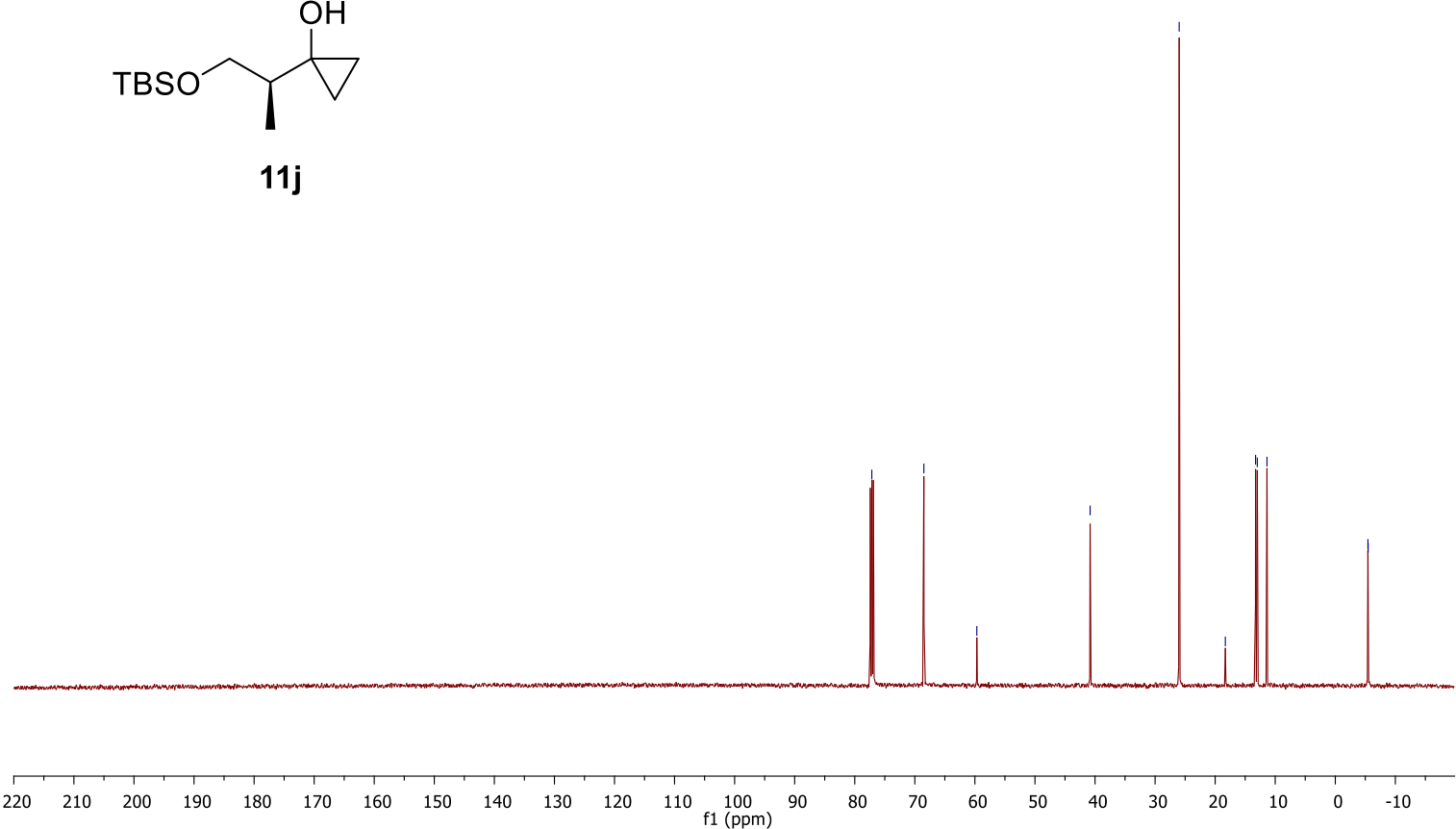

${ }^{13} \mathrm{C}$ NMR (126 MHz, $\mathrm{CDCl}_{3}$ ) of compound $\mathbf{1 1} \mathbf{j}$ 
<smiles>CCCCO[O+]SCCCCC1CC1(O)CCc1ccccc1</smiles>

$11 k$

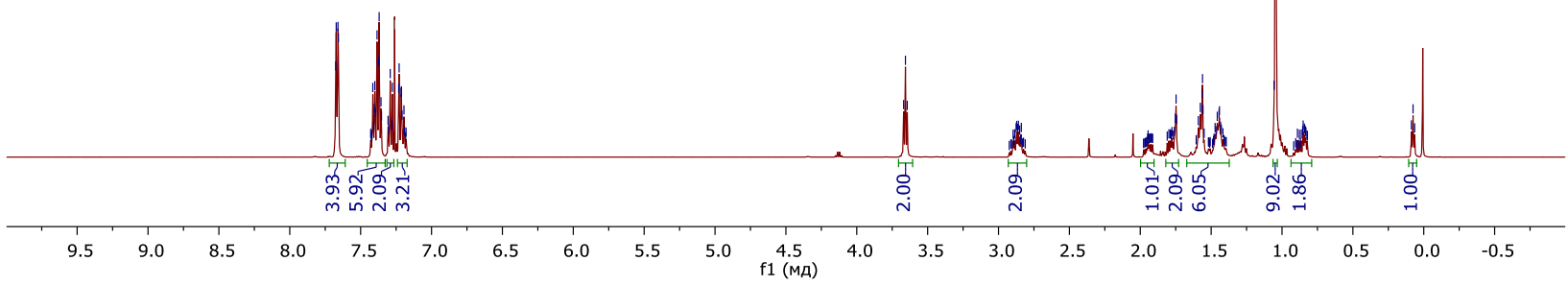

${ }^{1} \mathrm{H}$ NMR $\left(500 \mathrm{MHz}, \mathrm{CDCl}_{3}\right)$ of compound $\mathbf{1 1} \mathbf{k}$

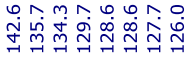

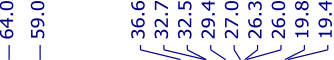<smiles>CCCCO[O+]SCCCCC1CC1(O)CCc1ccccc1</smiles>

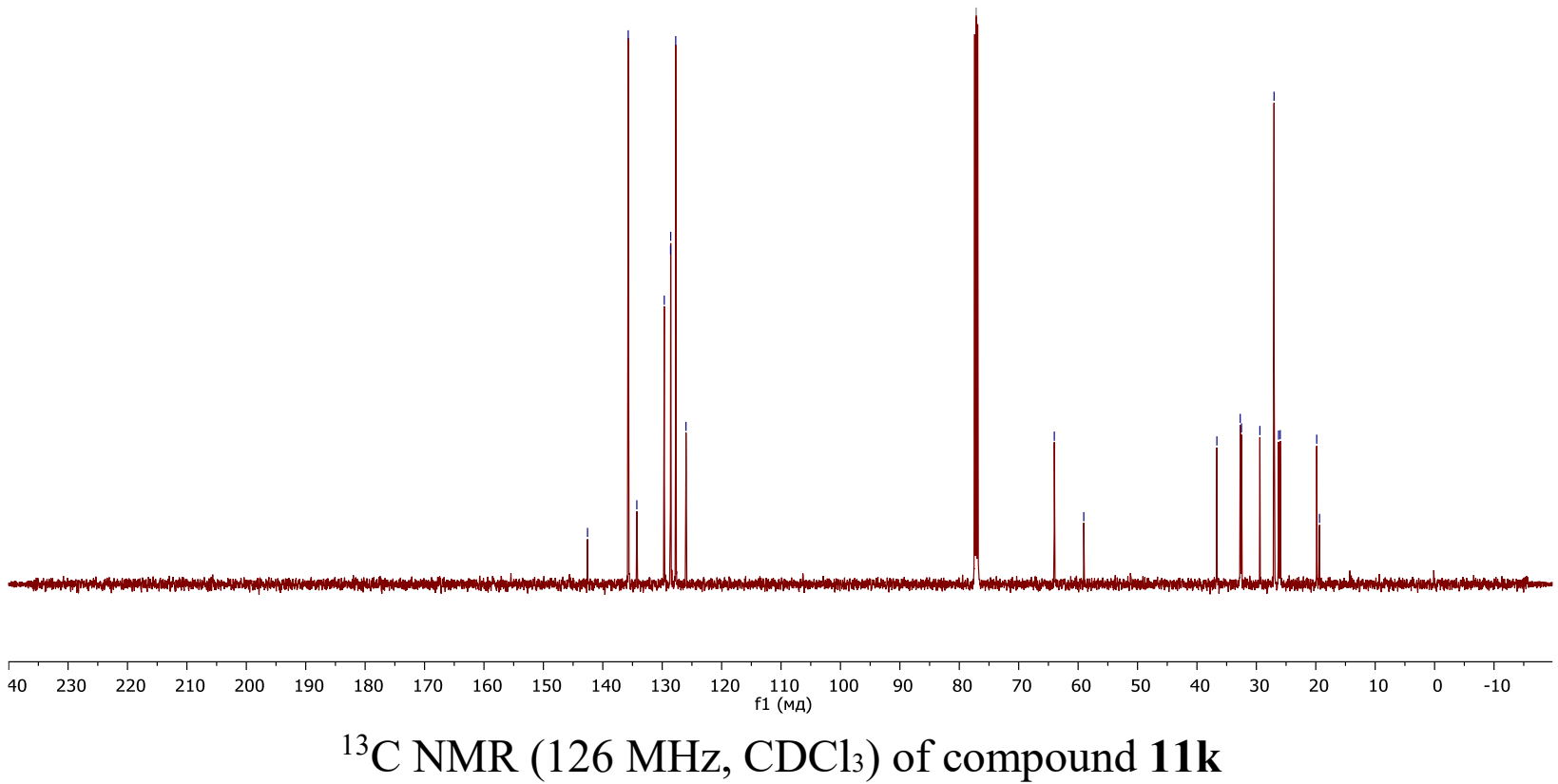


<smiles>CCC(O)CC1C[C@]1(O)CCc1ccccc1</smiles>

$( \pm)-\mathbf{S 1}$

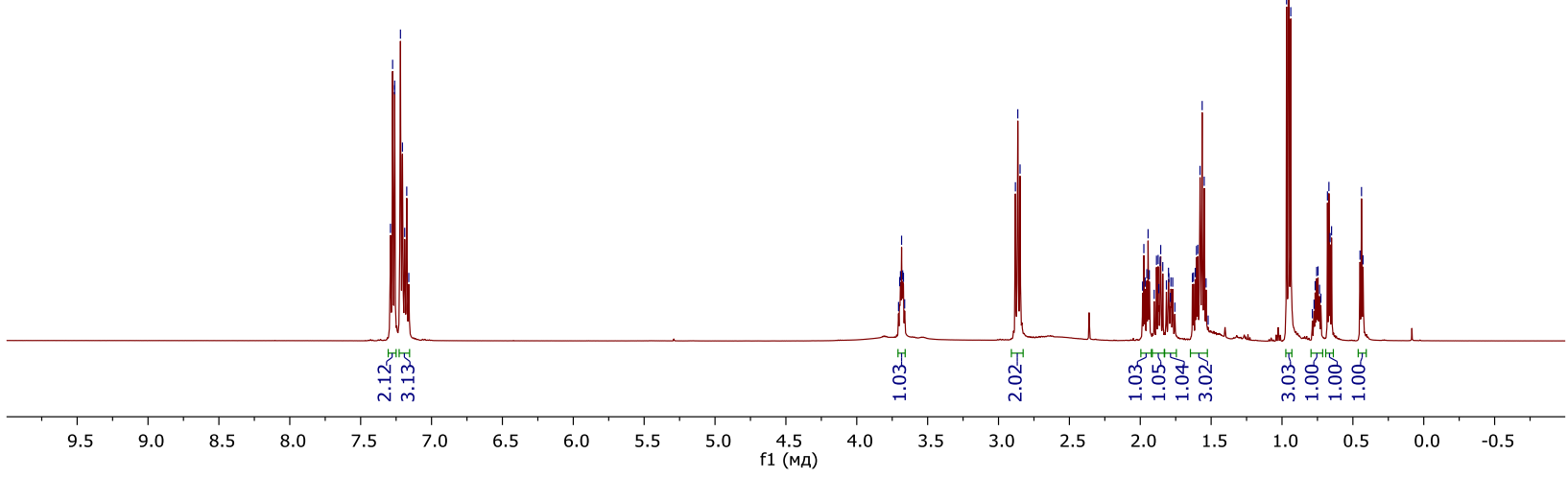

${ }^{1} \mathrm{H}$ NMR $\left(500 \mathrm{MHz}, \mathrm{CDCl}_{3}\right)$ of compound $\mathbf{S 1}$

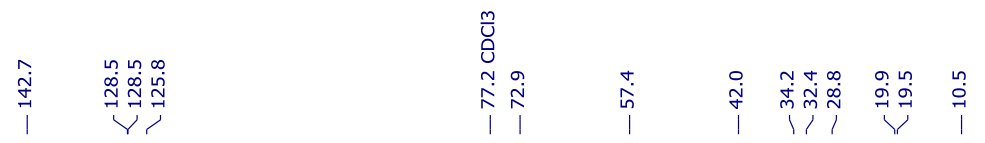<smiles>CCC(O)CC1C[C@]1(O)CCc1ccccc1</smiles>

$( \pm)-\mathbf{S 1}$

$\begin{array}{llllllllllll}30 & 220 & 210 & 200 & 190 & 180 & 170 & 160 & 150 & 140 & 130 & 120 \\ \text { f1 (MA) }\end{array}$

${ }^{13} \mathrm{C}$ NMR (126 MHz, $\left.\mathrm{CDCl}_{3}\right)$ of compound $\mathbf{S 1}$ 
<smiles>CCC(CC1C[C@]1(O)CCc1ccccc1)ON</smiles>

$( \pm)-111$

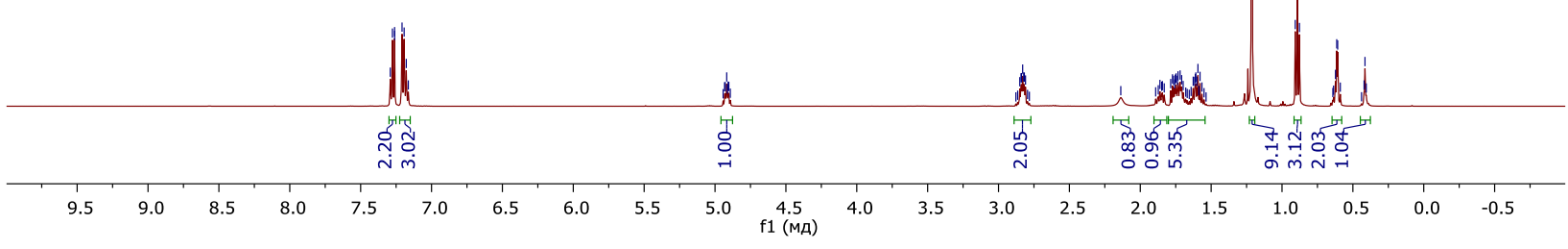

${ }^{1} \mathrm{H}$ NMR (500 MHz, $\left.\mathrm{CDCl}_{3}\right)$ of compound 111

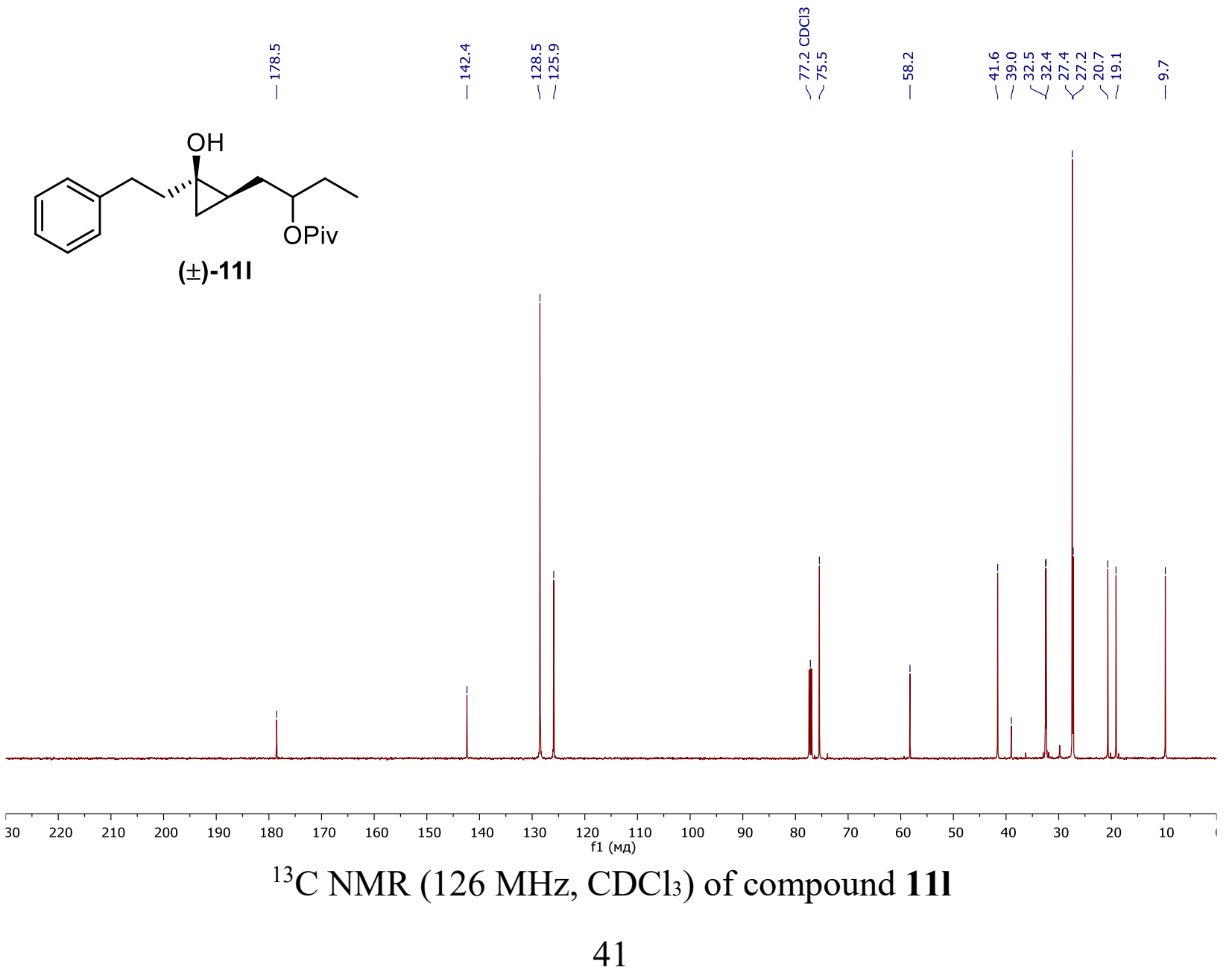




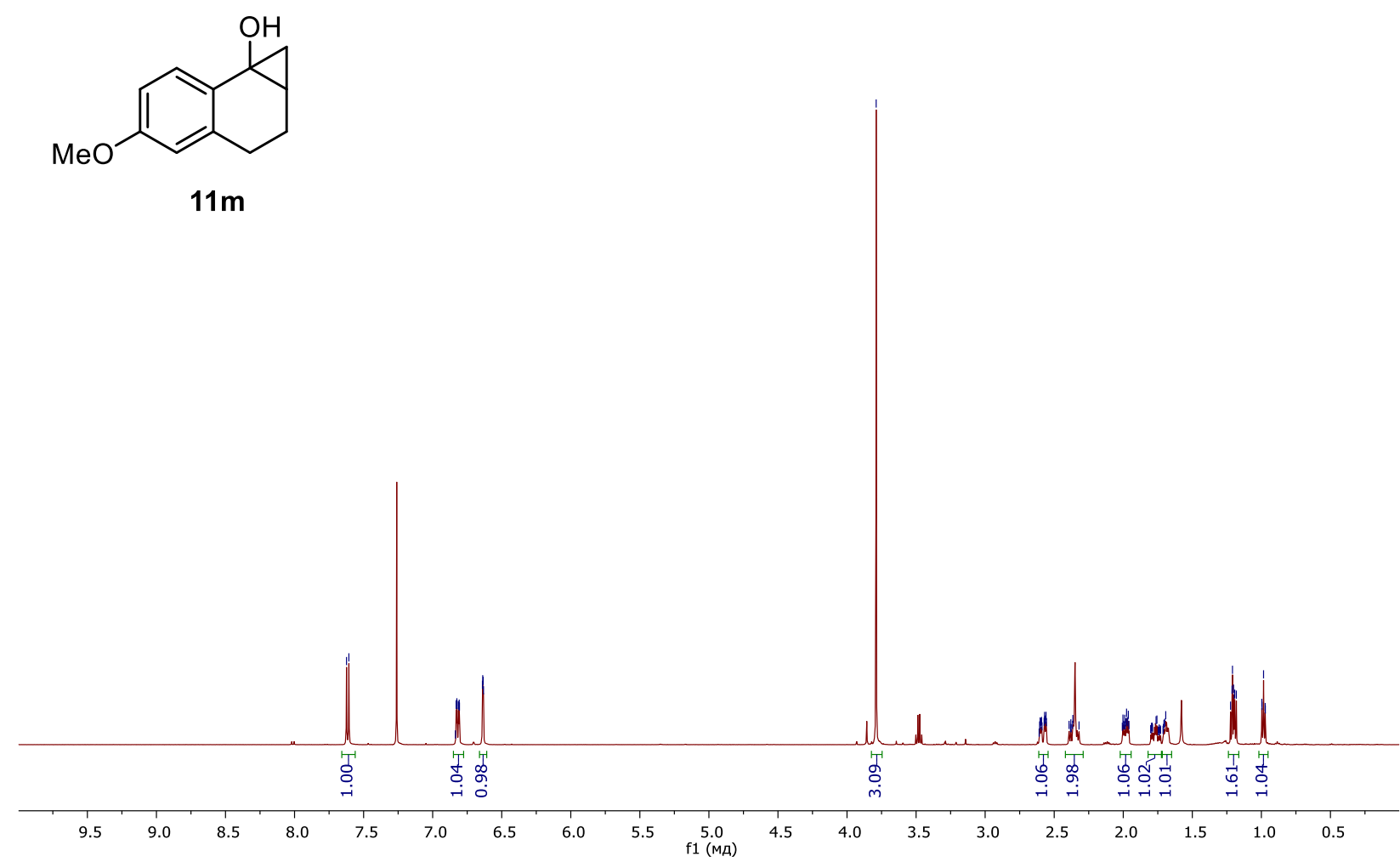

${ }^{1} \mathrm{H}$ NMR $\left(500 \mathrm{MHz}, \mathrm{CDCl}_{3}\right)$ of compound $11 \mathrm{~m}$

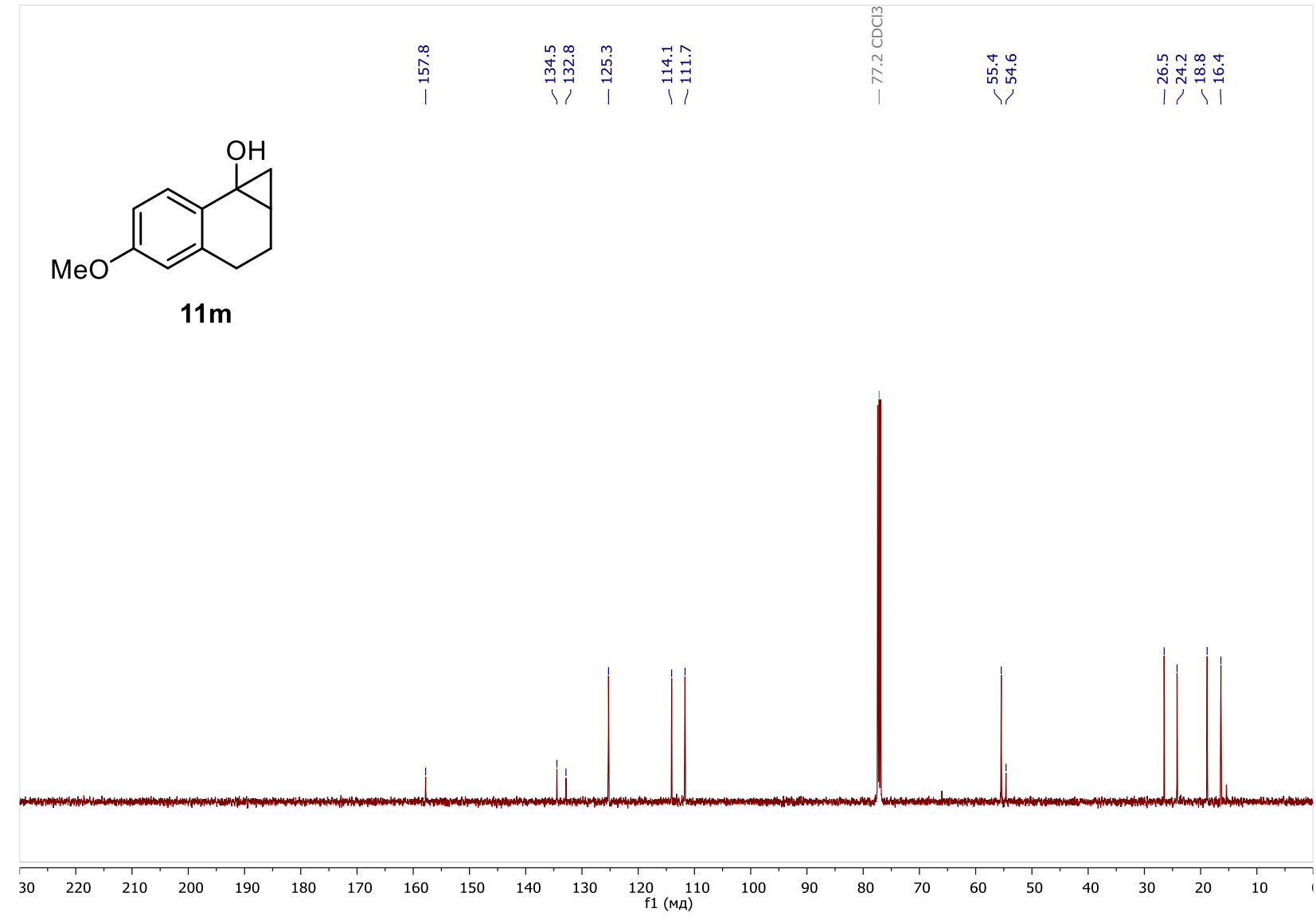

${ }^{13} \mathrm{C}$ NMR $\left(126 \mathrm{MHz}, \mathrm{CDCl}_{3}\right)$ of compound $\mathbf{1 1 m}$ 

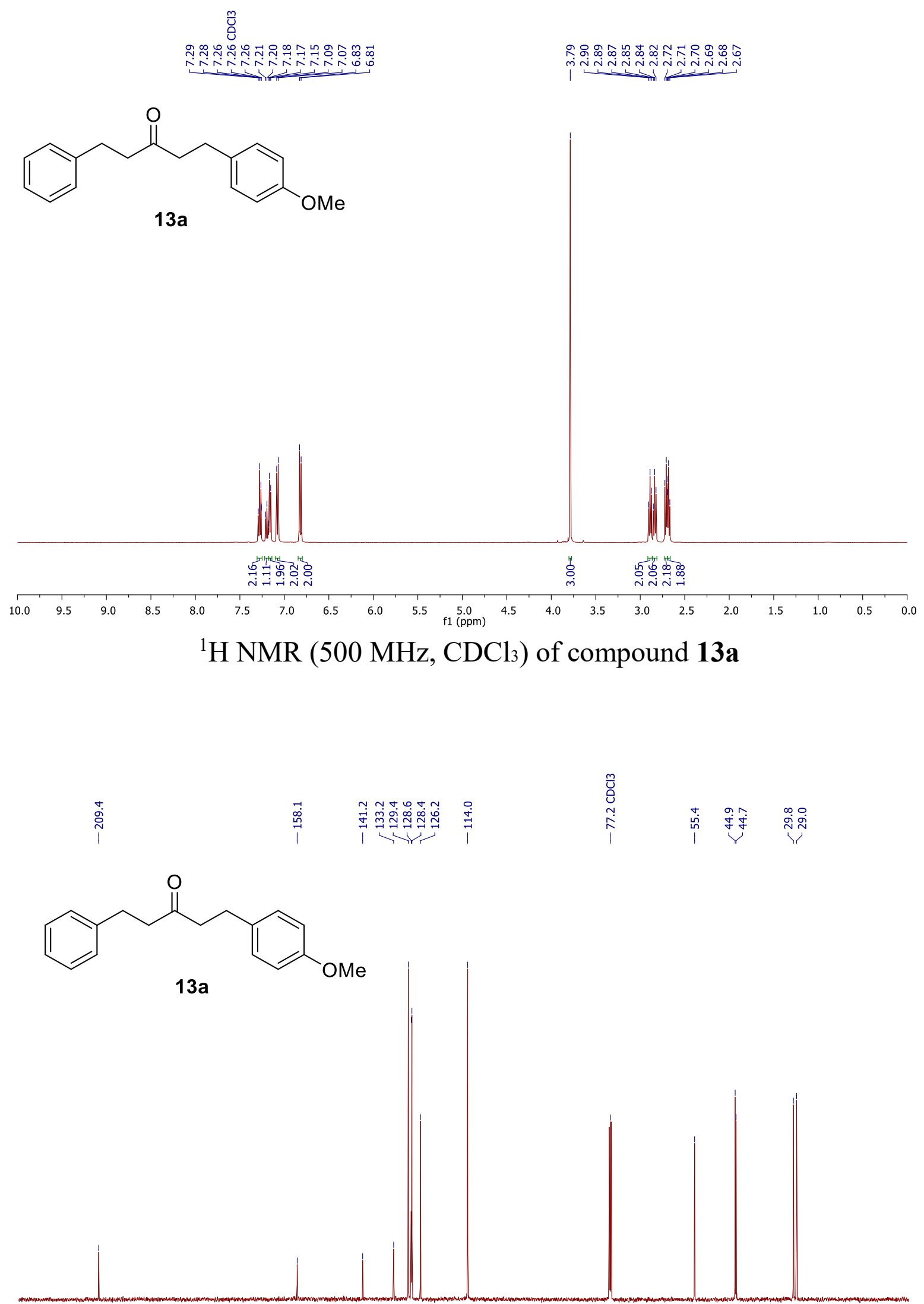

$\begin{array}{rlllllllllllllllllllllllll}230 & 220 & 210 & 200 & 190 & 180 & 170 & 160 & 150 & 140 & 130 & \underset{\mathrm{f} 1(\mathrm{ppm})}{120} & 110 & 100 & 90 & 80 & 70 & 60 & 50 & 40 & 30 & 20 & 10 & 0\end{array}$

${ }^{13} \mathrm{C}$ NMR (126 MHz, $\mathrm{CDCl}_{3}$ ) of compound 13a 

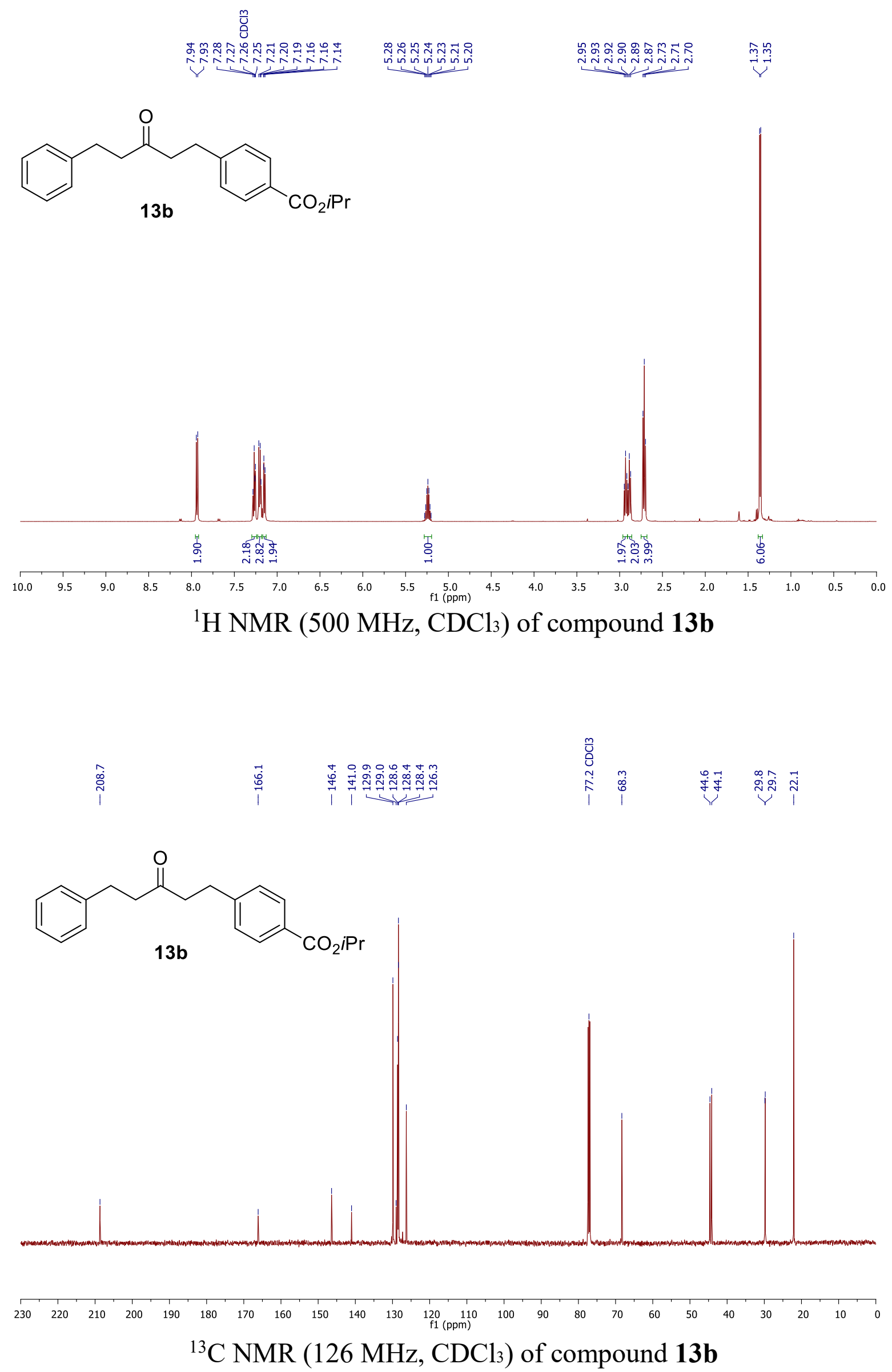

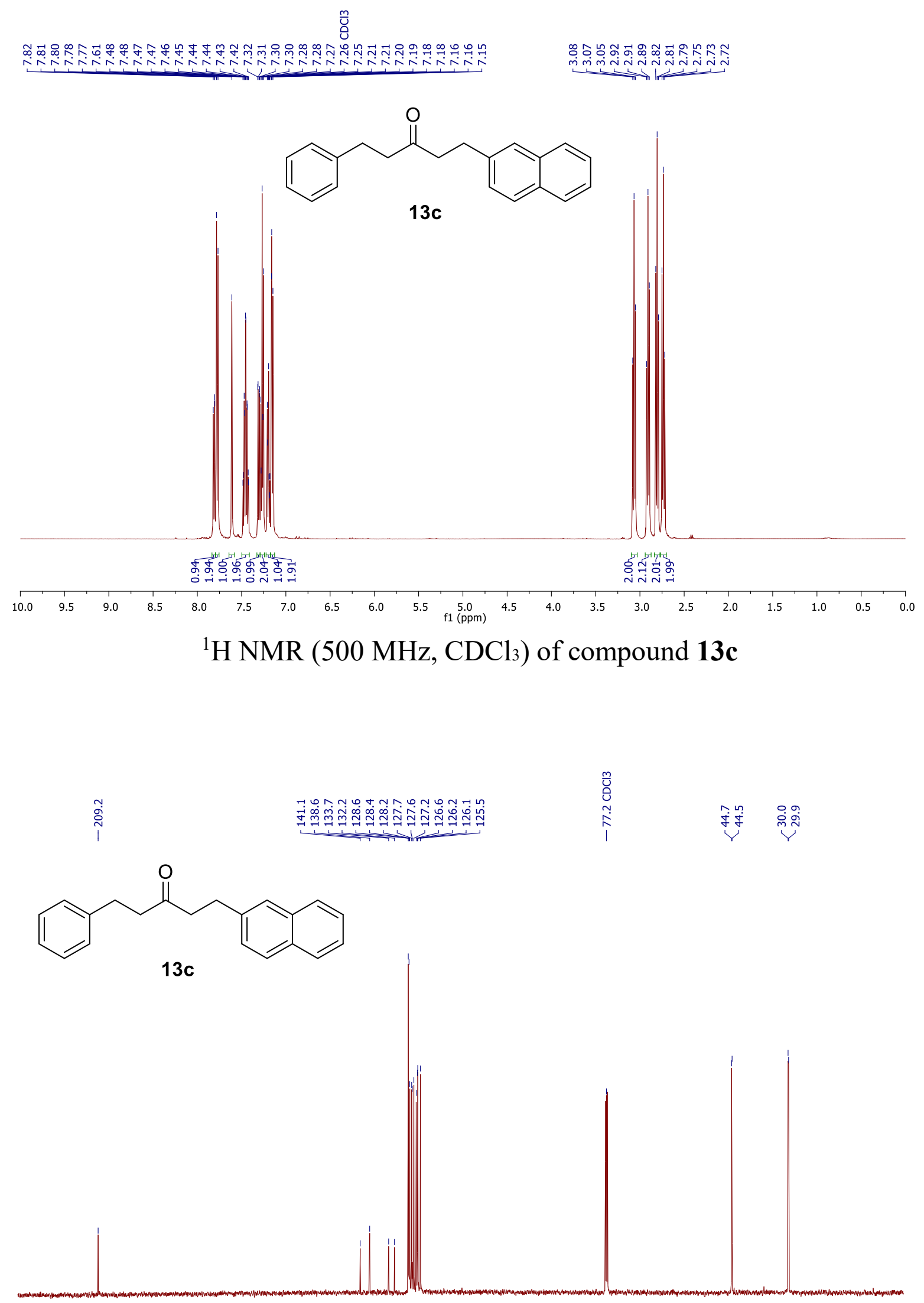

\begin{tabular}{rlllllllllllllllllllllllllllllll}
\hline 230 & 220 & 210 & 200 & 190 & 180 & 170 & 160 & 150 & 140 & 130 & 120 & 110 & 100 & 90 & 80 & 70 & 60 & 50 & 40 & 30 & 20 & 10 & 0
\end{tabular}

${ }^{13} \mathrm{C} \mathrm{NMR}\left(126 \mathrm{MHz}, \mathrm{CDCl}_{3}\right)$ of compound $\mathbf{1 3 c}$ 

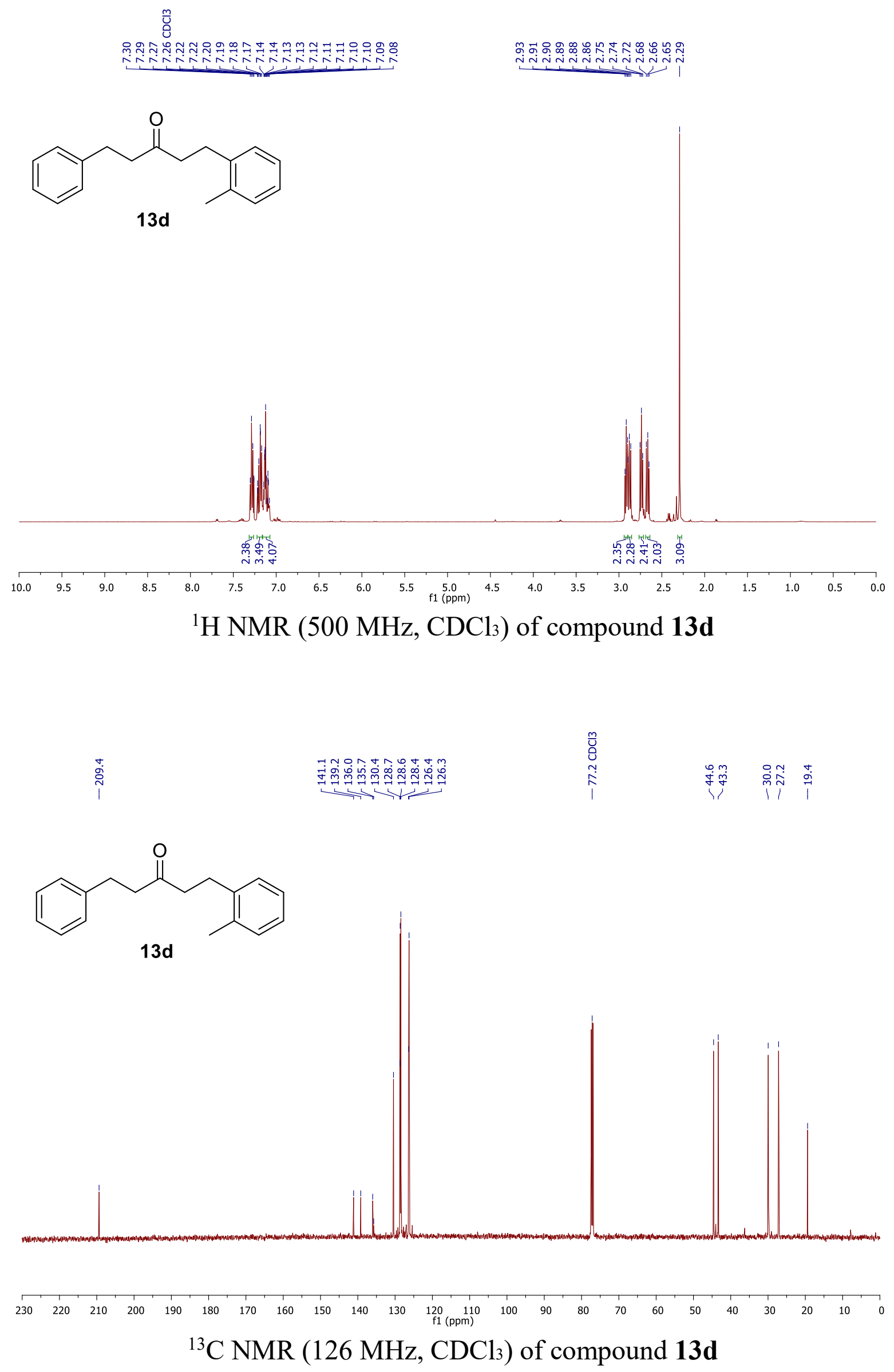
<smiles>CCCOC(=O)Cc1ccccc1CCC(=O)CCc1ccccc1</smiles>

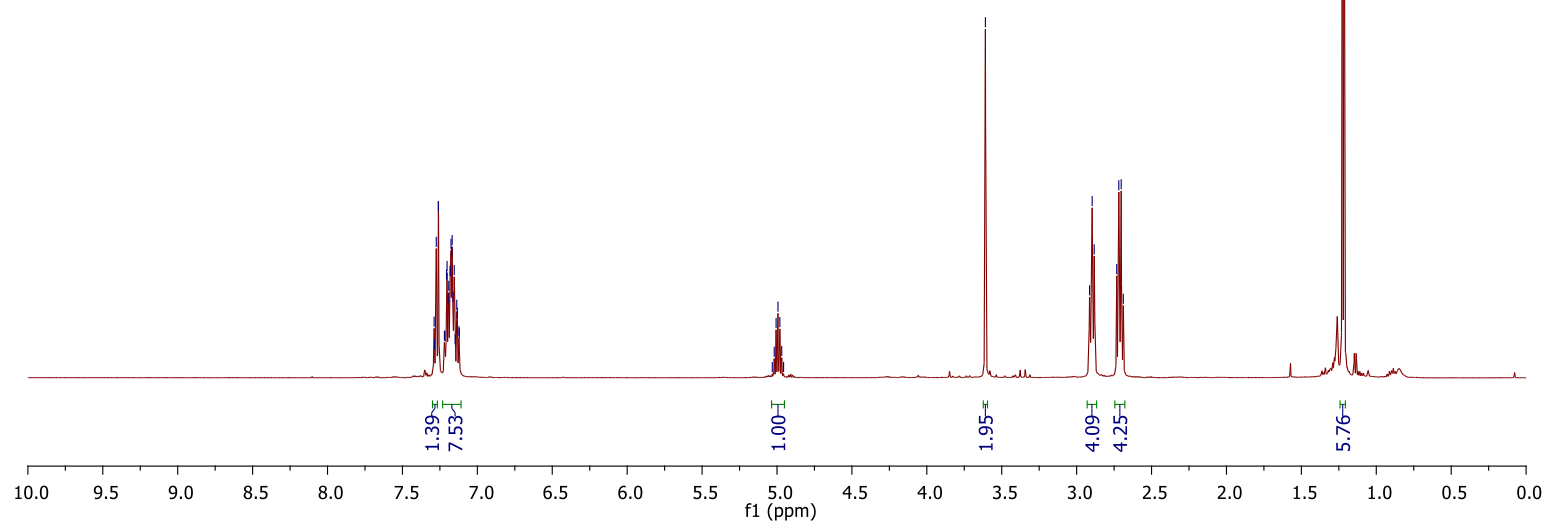

${ }^{1} \mathrm{H}$ NMR $\left(500 \mathrm{MHz}, \mathrm{CDCl}_{3}\right)$ of compound $\mathbf{1 3 e}$

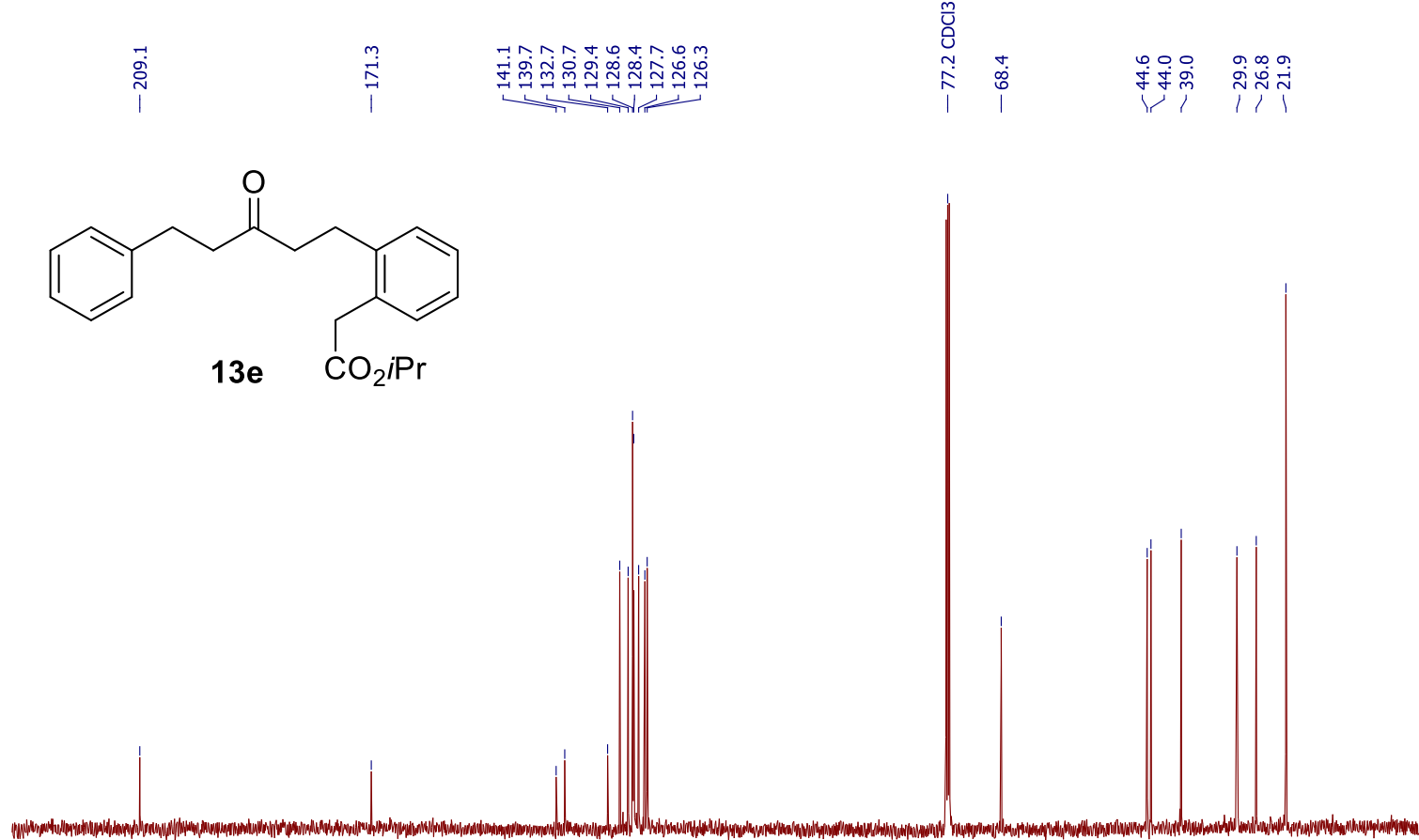

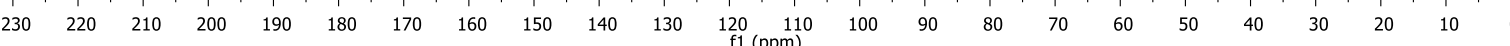

${ }^{13} \mathrm{C}$ NMR (126 MHz, $\mathrm{CDCl}_{3}$ ) of compound 13e 


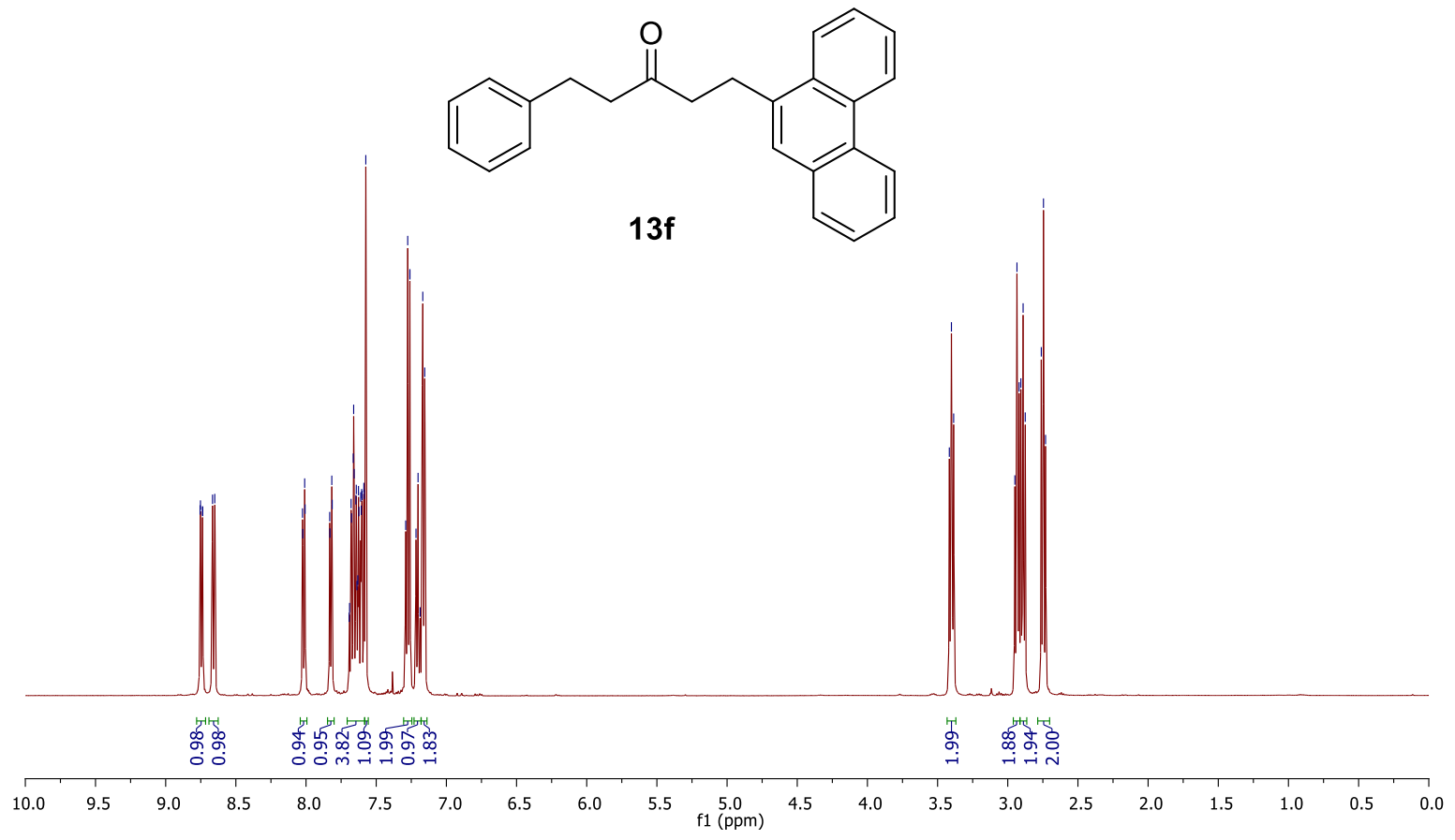

${ }^{1} \mathrm{H}$ NMR (500 MHz, $\left.\mathrm{CDCl}_{3}\right)$ of compound $\mathbf{1 3 f}$

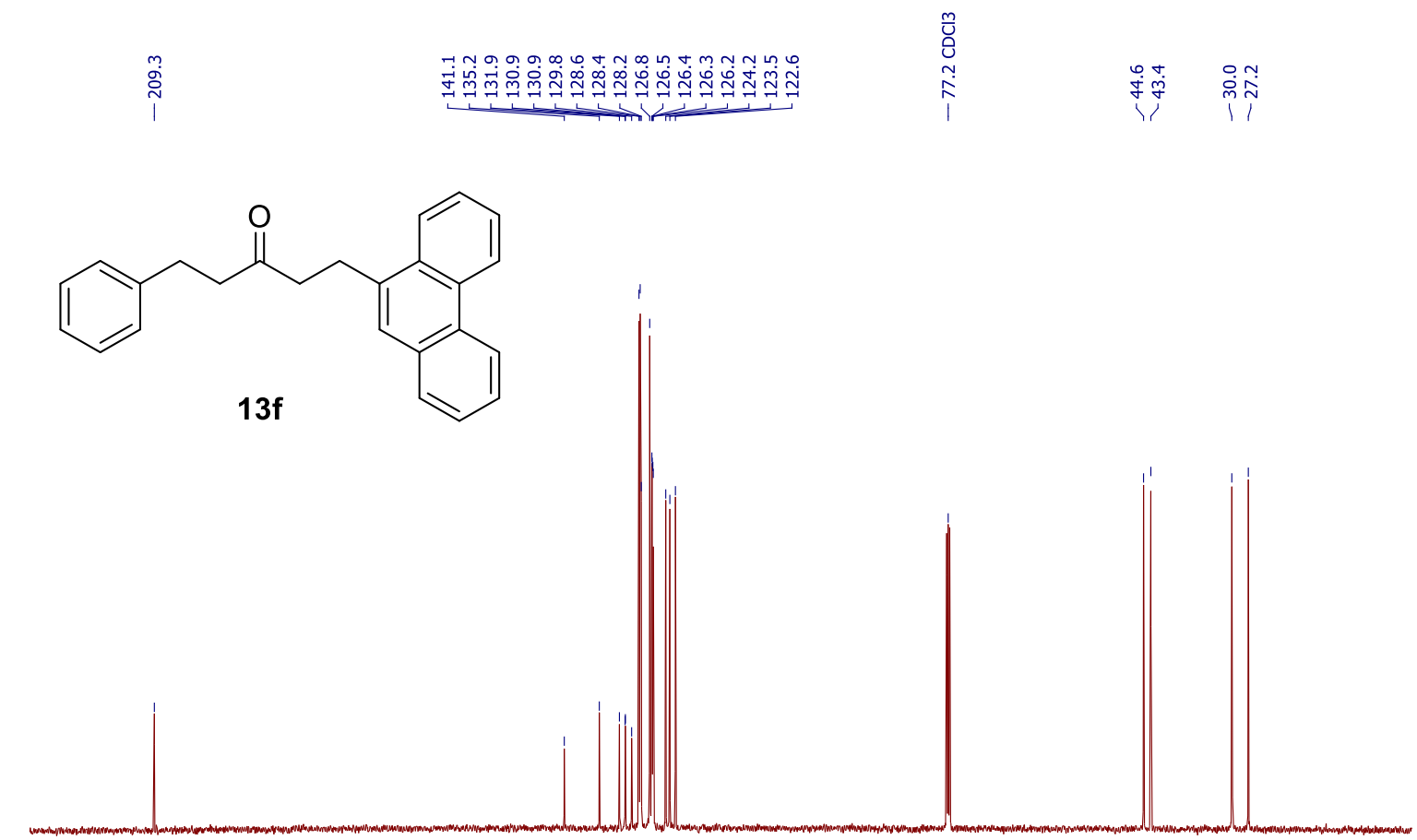

$\begin{array}{lllllllllllllllllllllllll}230 & 220 & 210 & 200 & 190 & 180 & 170 & 160 & 150 & 140 & 130 & \underset{120}{120}(\mathrm{pl}) & 100 & 90 & 80 & 70 & 60 & 50 & 40 & 30 & 20 & 10 & 0\end{array}$

${ }^{13} \mathrm{C}$ NMR $\left(126 \mathrm{MHz}, \mathrm{CDCl}_{3}\right)$ of compound $\mathbf{1 3 f}$ 
<smiles>COc1ccc(CCC(=O)CCCOS(=O)(=O)[Mg]C(C)(C)C)cc1</smiles>

$13 \mathrm{~g}$

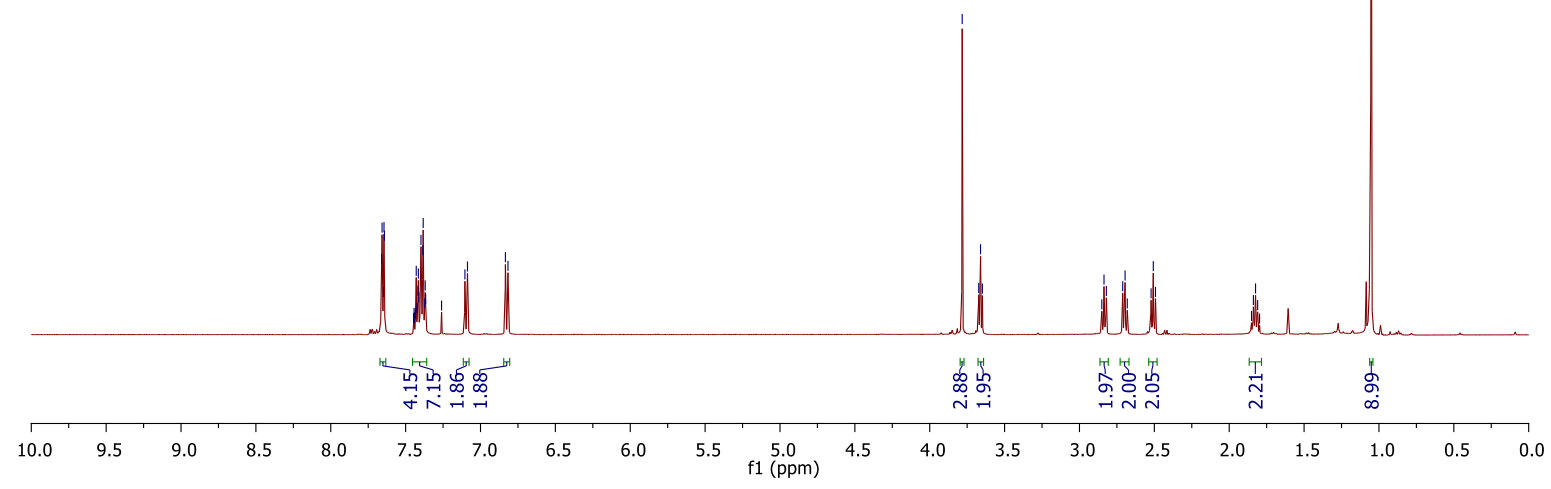

${ }^{1} \mathrm{H}$ NMR $\left(500 \mathrm{MHz}, \mathrm{CDCl}_{3}\right)$ of compound $\mathbf{1 3 g}$
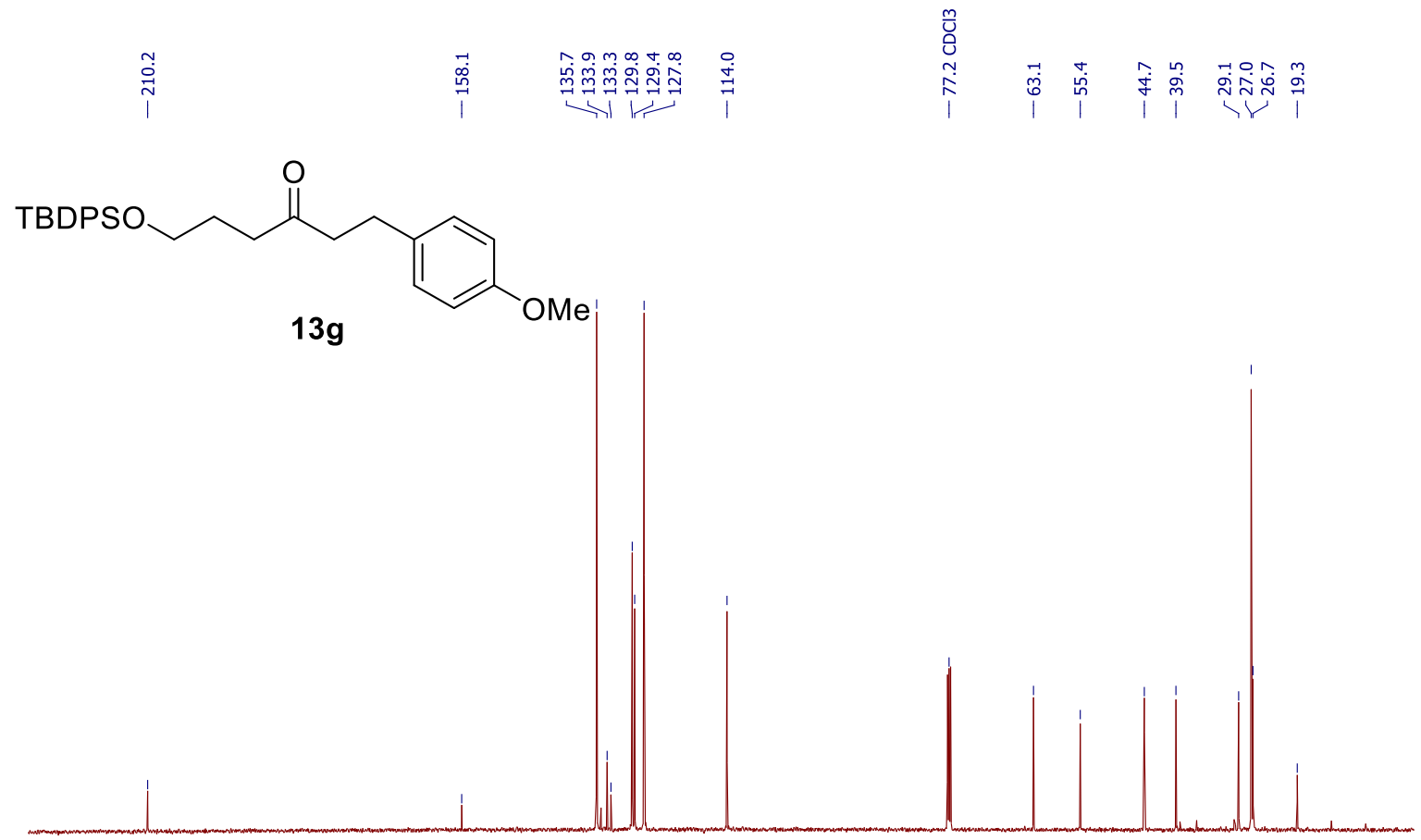

$\begin{array}{llllllllllllllllllllllll}230 & 220 & 210 & 200 & 190 & 180 & 170 & 160 & 150 & 140 & 130 & \left.\begin{array}{l}120 \\ \mathrm{f} 1(\mathrm{ppm})\end{array}\right) & 100 & 90 & 80 & 70 & 60 & 50 & 40 & 30 & 20 & 10\end{array}$

${ }^{13} \mathrm{C}$ NMR $\left(126 \mathrm{MHz}, \mathrm{CDCl}_{3}\right)$ of compound $\mathbf{1 3 g}$ 

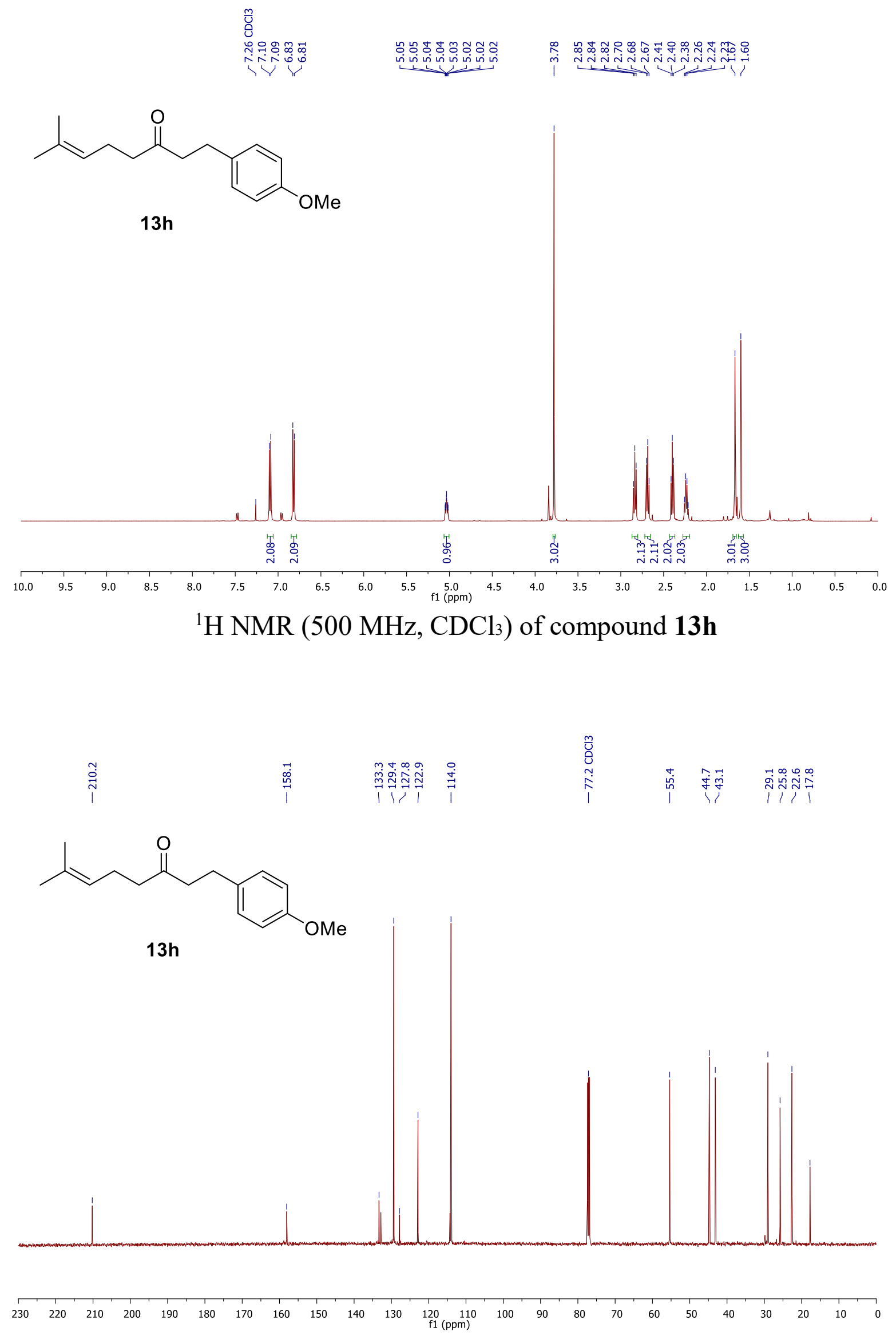

${ }^{13} \mathrm{C}$ NMR (126 MHz, $\mathrm{CDCl}_{3}$ ) of compound $\mathbf{1 3 h}$ 


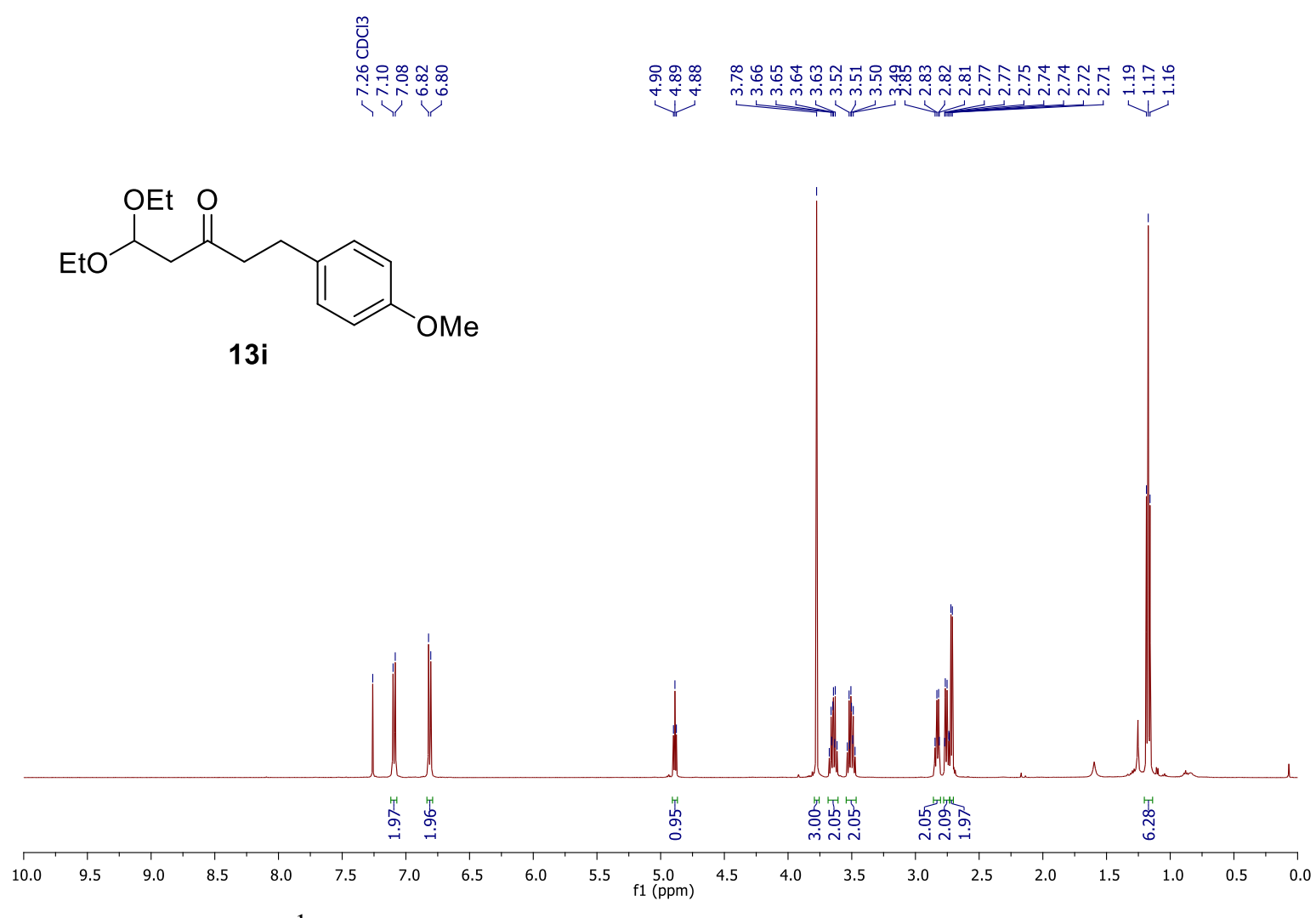

${ }^{1} \mathrm{H}$ NMR (500 MHz, $\mathrm{CDCl}_{3}$ ) of compound $\mathbf{1 3 i}$

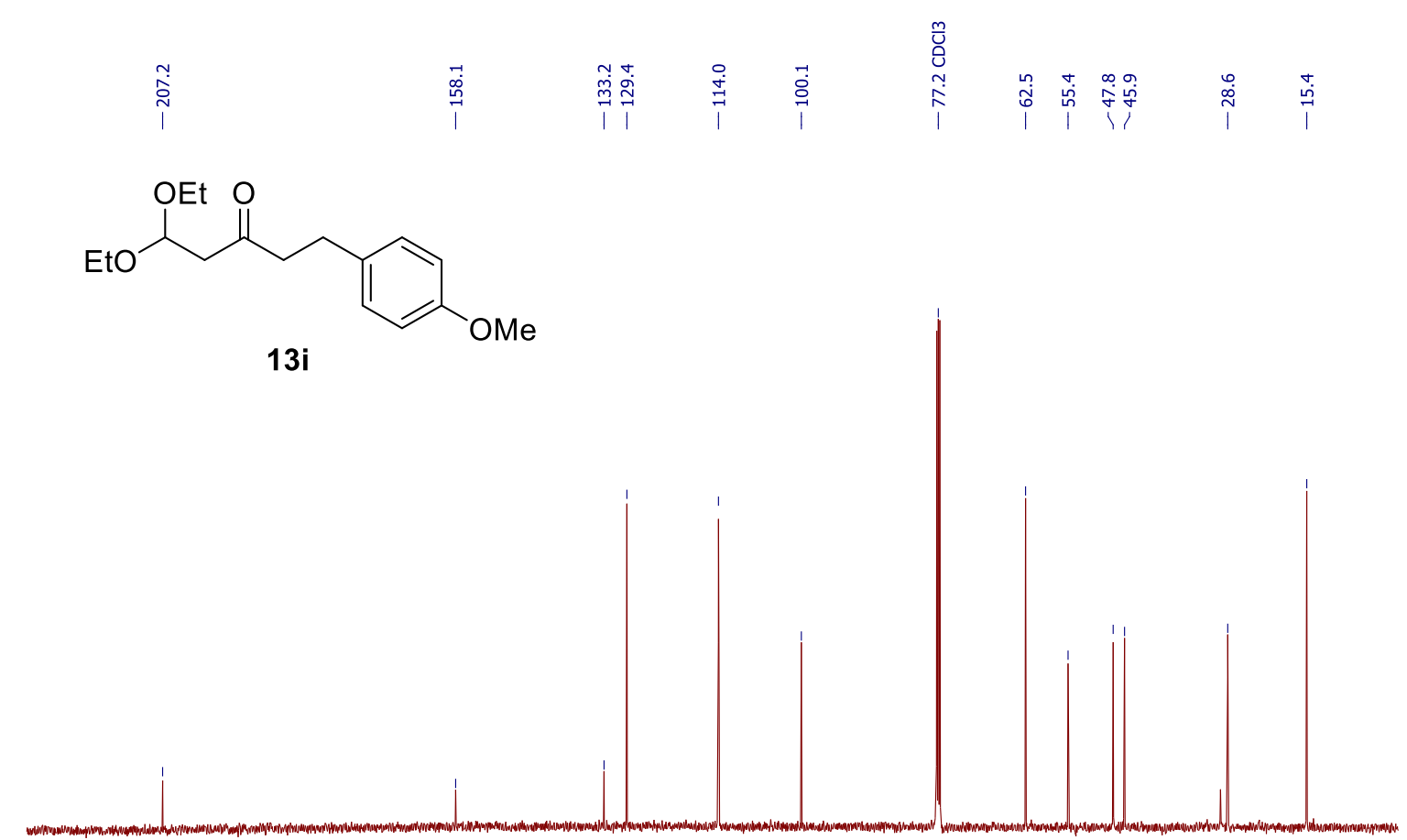

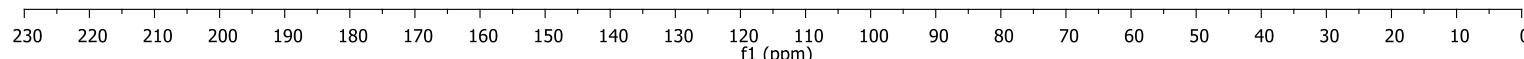

${ }^{13} \mathrm{C}$ NMR (126 MHz, $\mathrm{CDCl}_{3}$ ) of compound 13i 


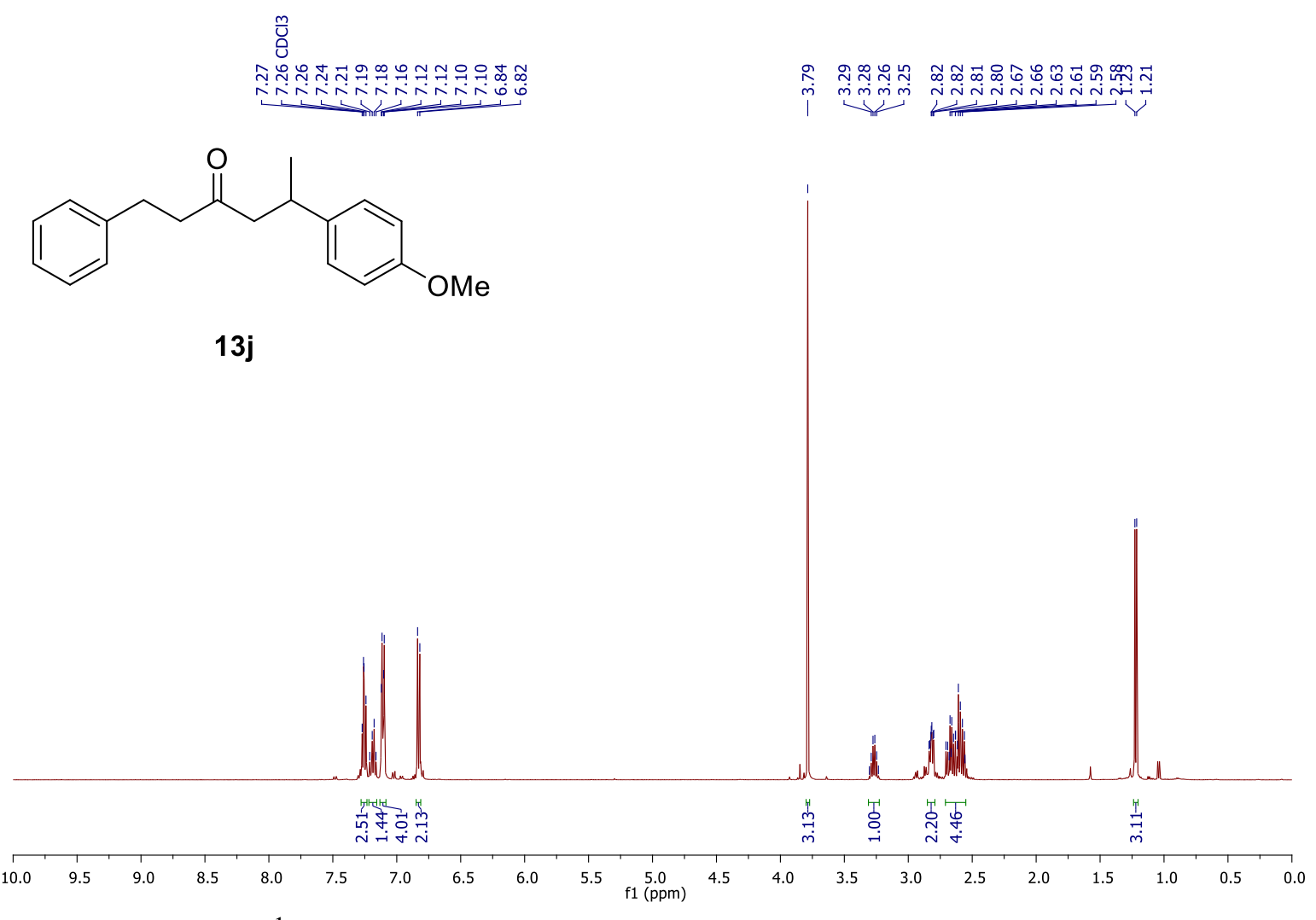

${ }^{1} \mathrm{H}$ NMR $\left(500 \mathrm{MHz}, \mathrm{CDCl}_{3}\right)$ of compound $\mathbf{1 3} \mathbf{j}$

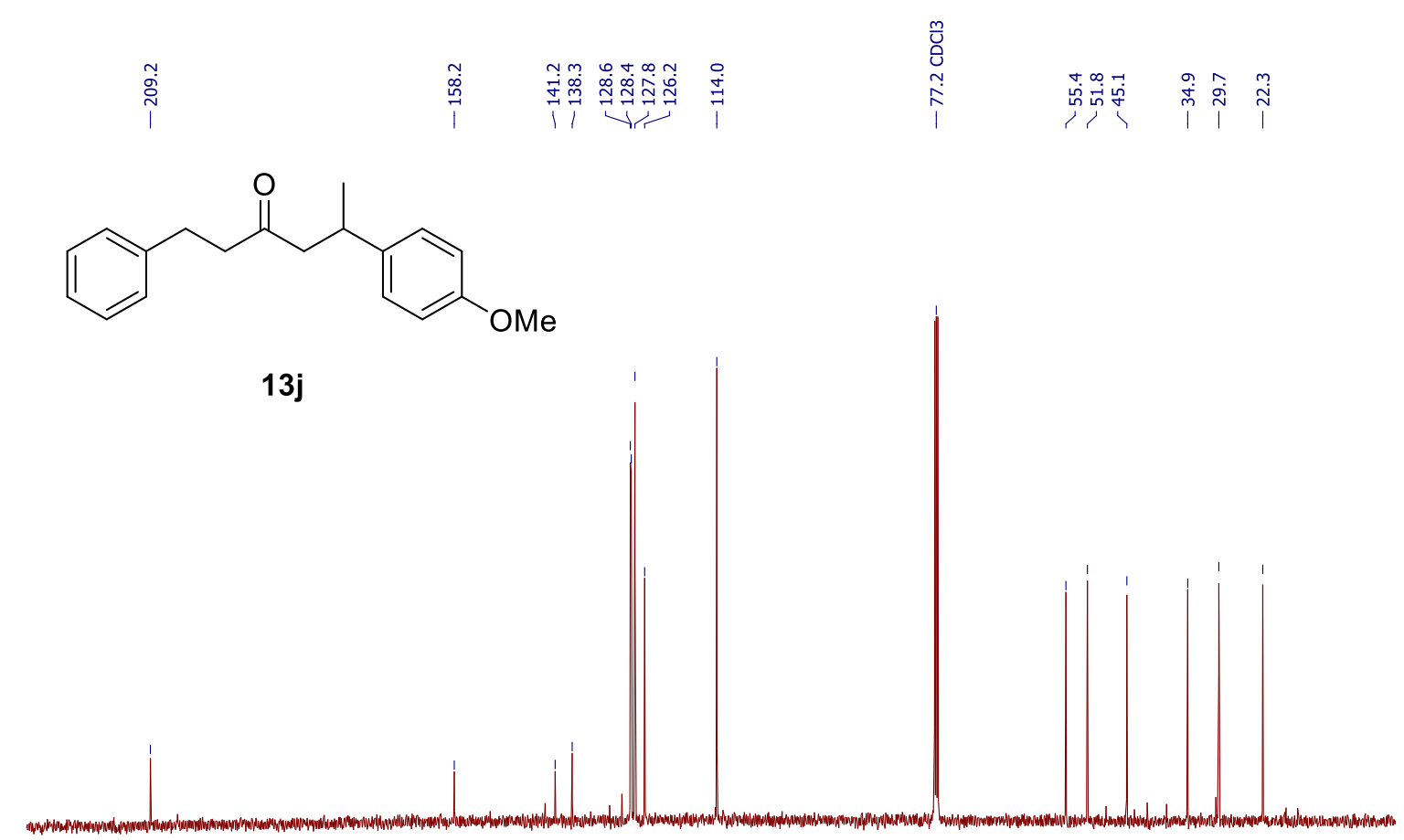

\begin{tabular}{rlllllllllllllllllllllll}
\hline 230 & 220 & 210 & 200 & 190 & 180 & 170 & 160 & 150 & 140 & 130 & $\underset{\mathrm{f} 1}{120}(\mathrm{ppm})$ & 110 & 100 & 90 & 80 & 70 & 60 & 50 & 40 & 30 & 20 & 10 &
\end{tabular}

${ }^{13} \mathrm{C} \mathrm{NMR}\left(126 \mathrm{MHz}, \mathrm{CDCl}_{3}\right)$ of compound $\mathbf{1 3} \mathbf{j}$ 

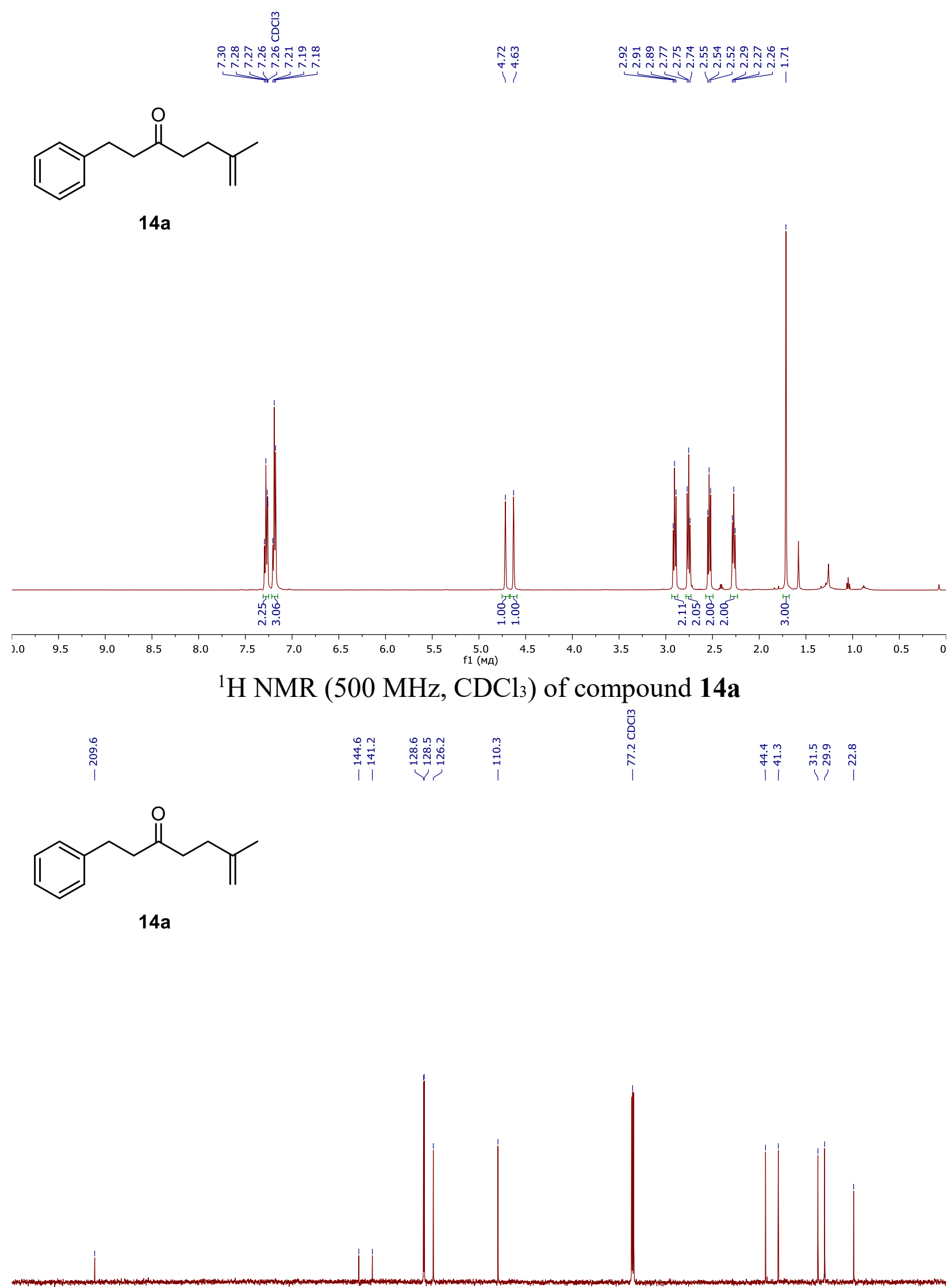

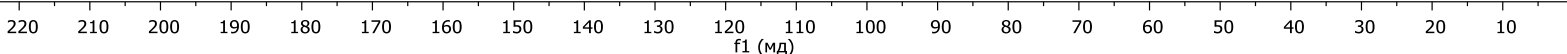

${ }^{13} \mathrm{C}$ NMR (126 MHz, $\left.\mathrm{CDCl}_{3}\right)$ of compound 14a 


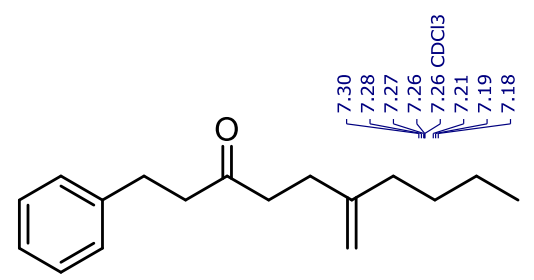

$14 b$

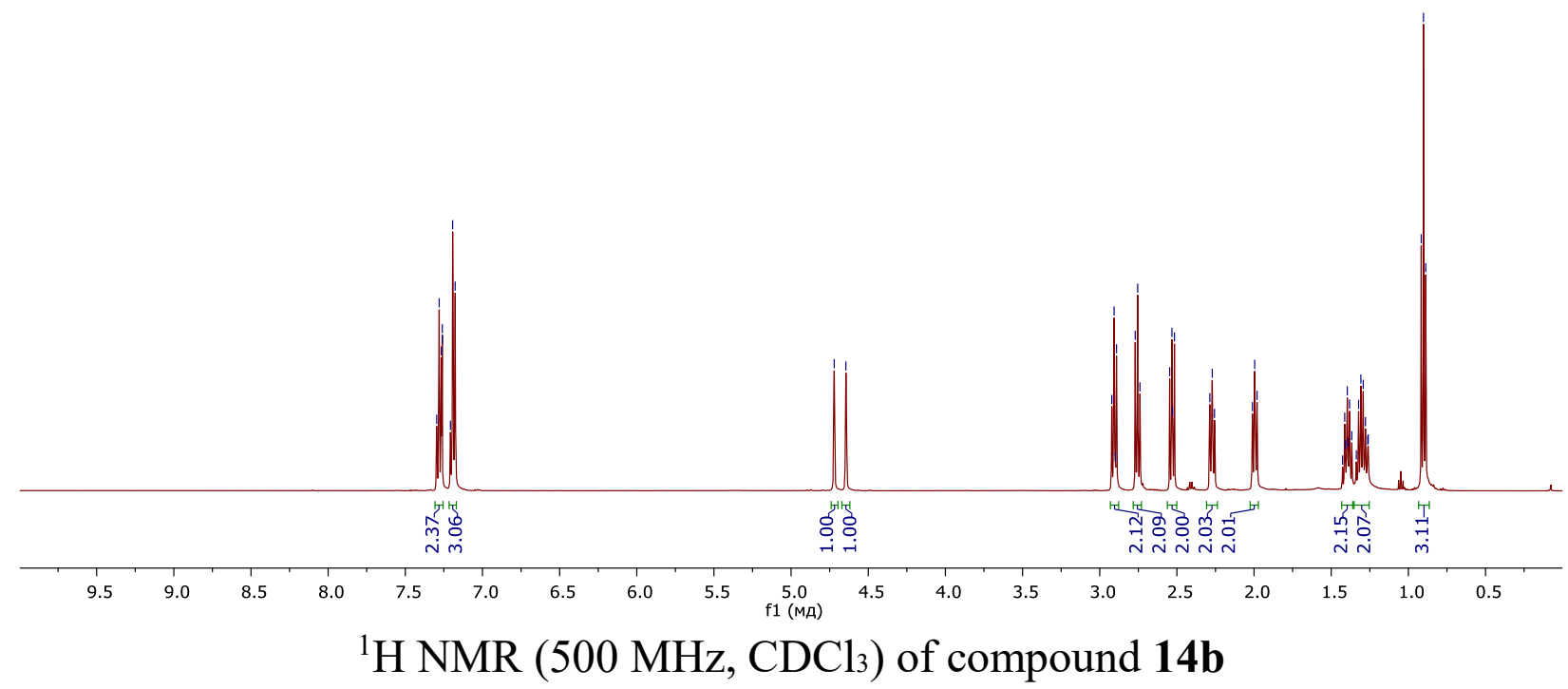<smiles>C=C(CCCC)CCC(=O)CCc1ccccc1</smiles>

$14 b$

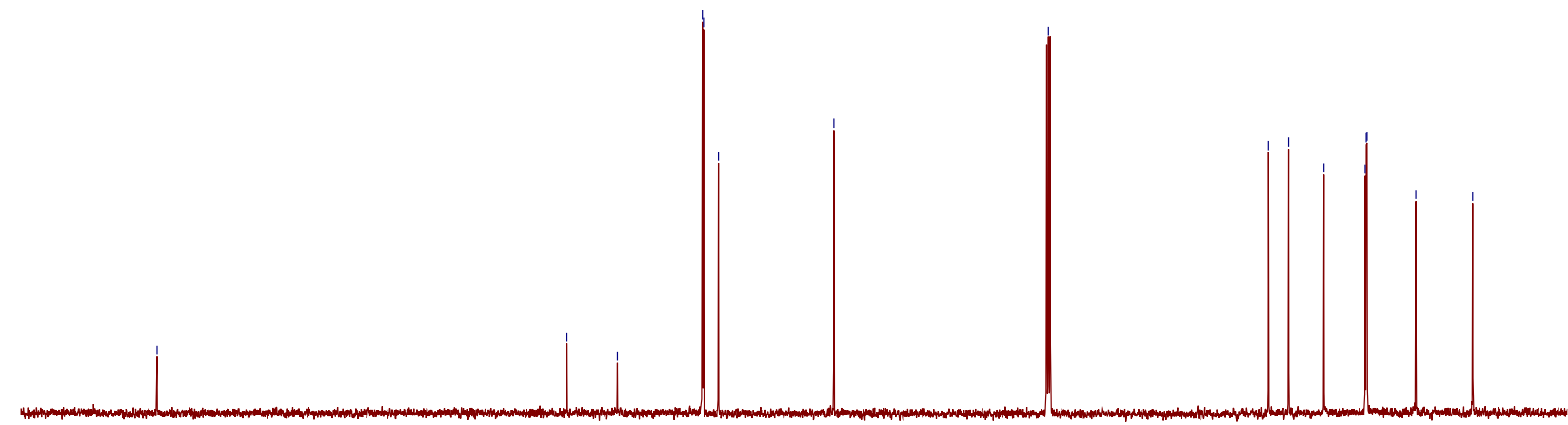

$\begin{array}{llllllllllllllllllllllll}1 & 220 & 210 & 200 & 190 & 180 & 170 & 160 & 150 & 140 & 130 & \underset{f 1}{120}(\mathrm{MA}) & 110 & 100 & 90 & 80 & 70 & 60 & 50 & 40 & 30 & 20 & 10\end{array}$

${ }^{13} \mathrm{C} \mathrm{NMR}\left(126 \mathrm{MHz}, \mathrm{CDCl}_{3}\right)$ of compound $\mathbf{1 4 b}$ 

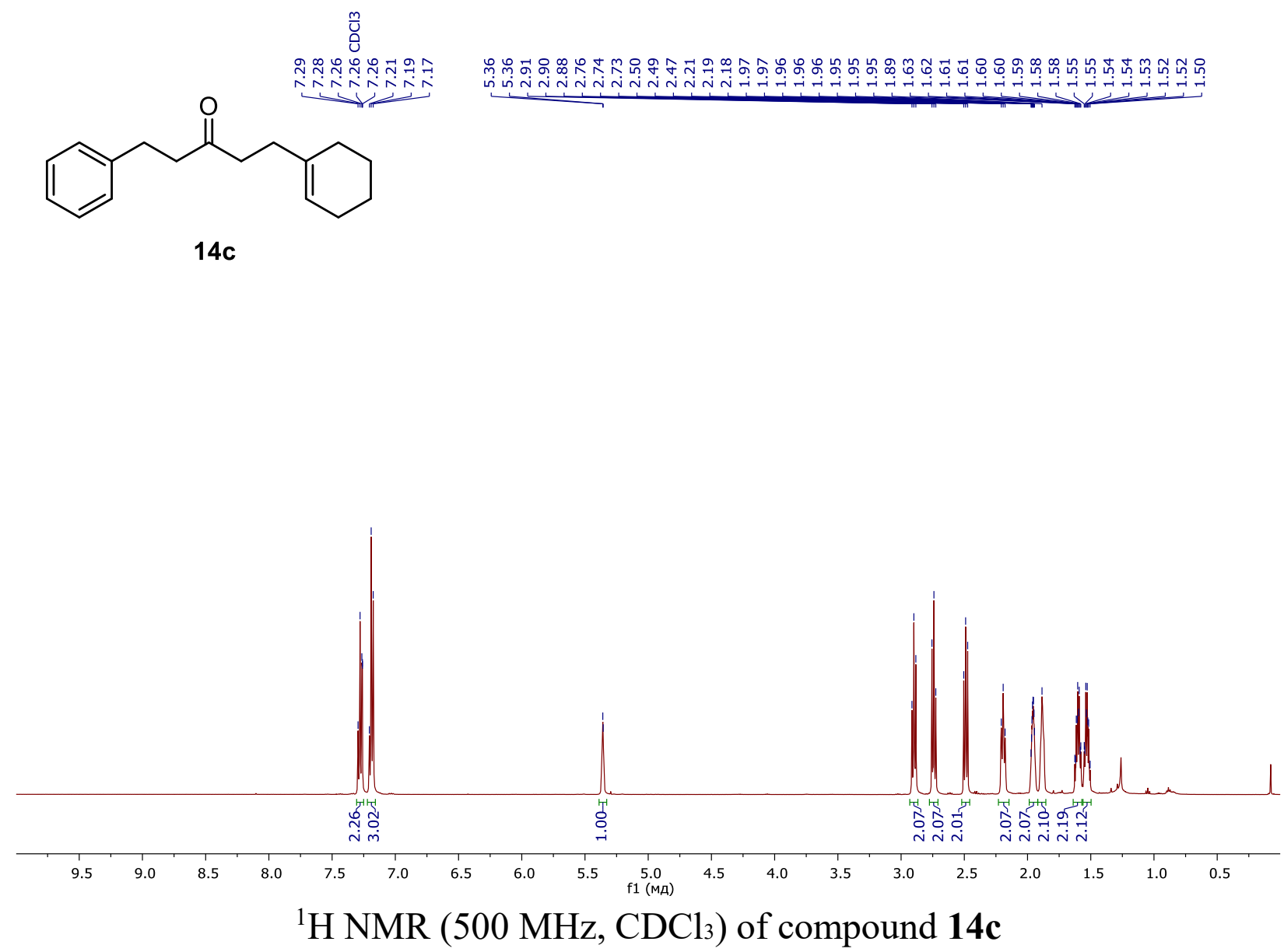
i.

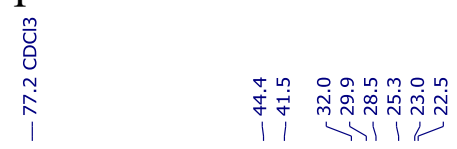<smiles>O=C(CCC1=CCCCC1)CCc1ccccc1</smiles>

$14 c$

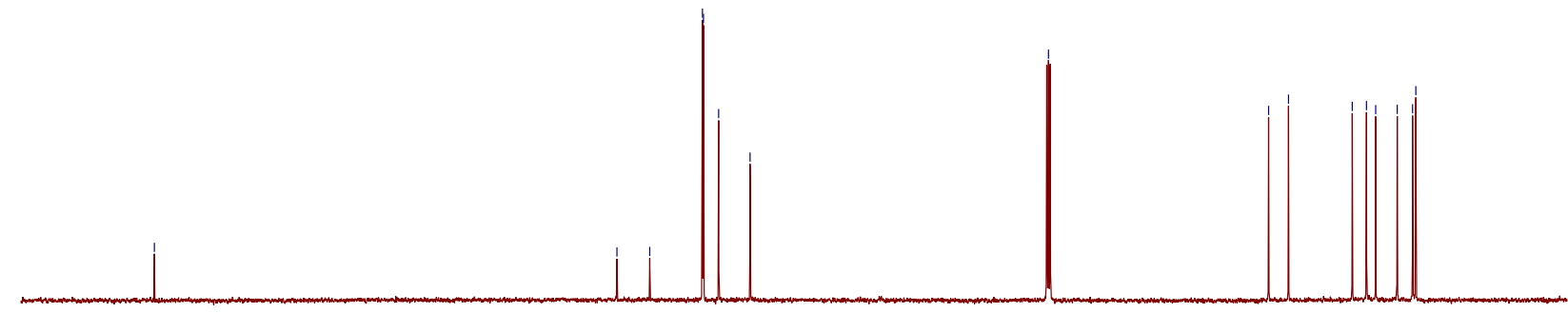

$\begin{array}{llllllllllllllllllllllllllllllll}30 & 220 & 210 & 200 & 190 & 180 & 170 & 160 & 150 & 140 & 130 & 120 & 110 & 100 & 90 & 80 & 70 & 60 & 50 & 40 & 30 & 20 & 10\end{array}$ ${ }^{13} \mathrm{C}$ NMR (126 MHz, $\left.\mathrm{CDCl}_{3}\right)$ of compound $\mathbf{1 4 c}$ 

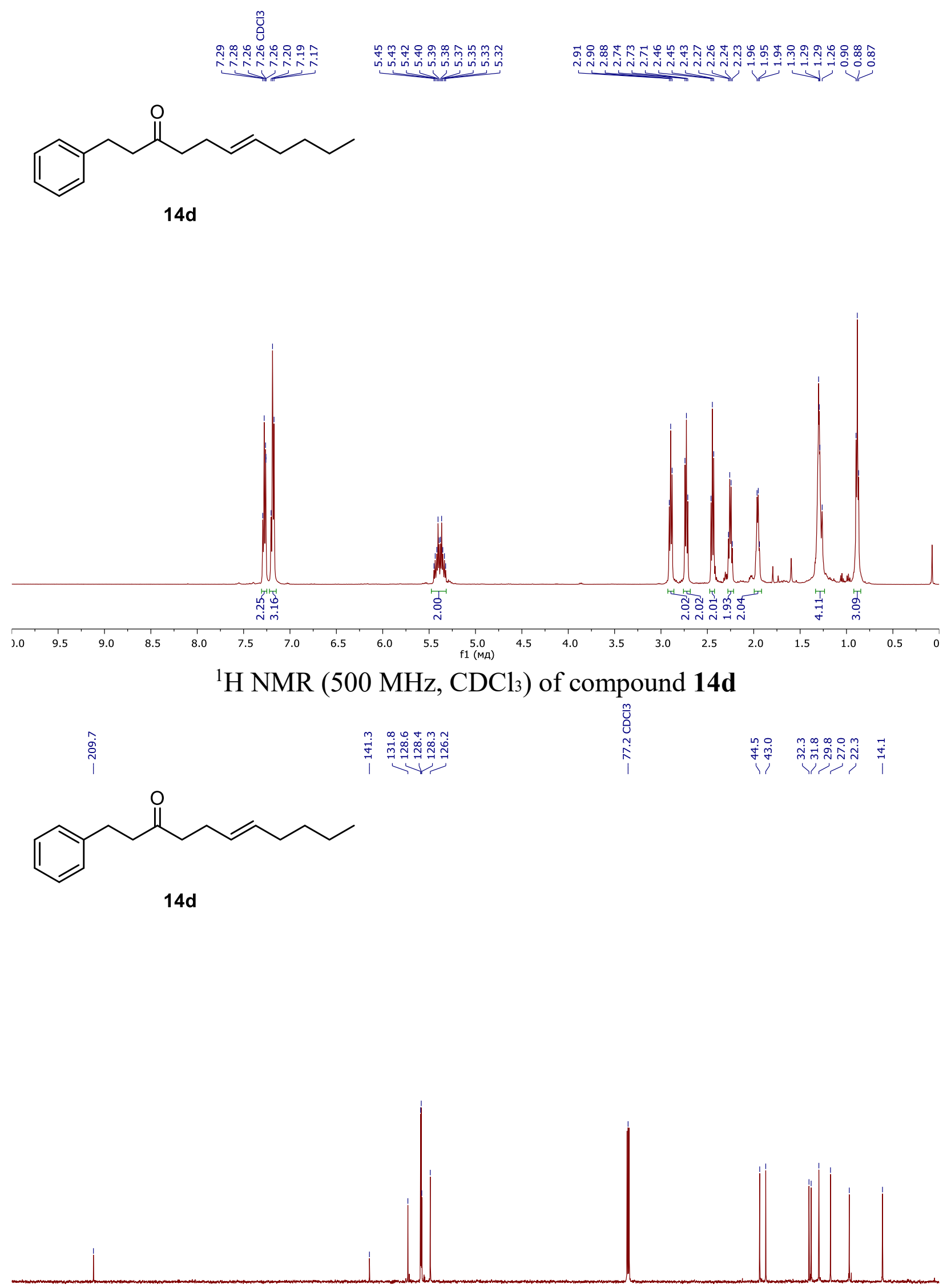

$\begin{array}{llllllllllllllllllllllll}30 & 220 & 210 & 200 & 190 & 180 & 170 & 160 & 150 & 140 & 130 & 120 & 110 & 100 & 90 & 80 & 70 & 60 & 50 & 40 & 30 & 20 & 10 & \end{array}$

${ }^{13} \mathrm{C}$ NMR (126 MHz, $\left.\mathrm{CDCl}_{3}\right)$ of compound $14 d$ 

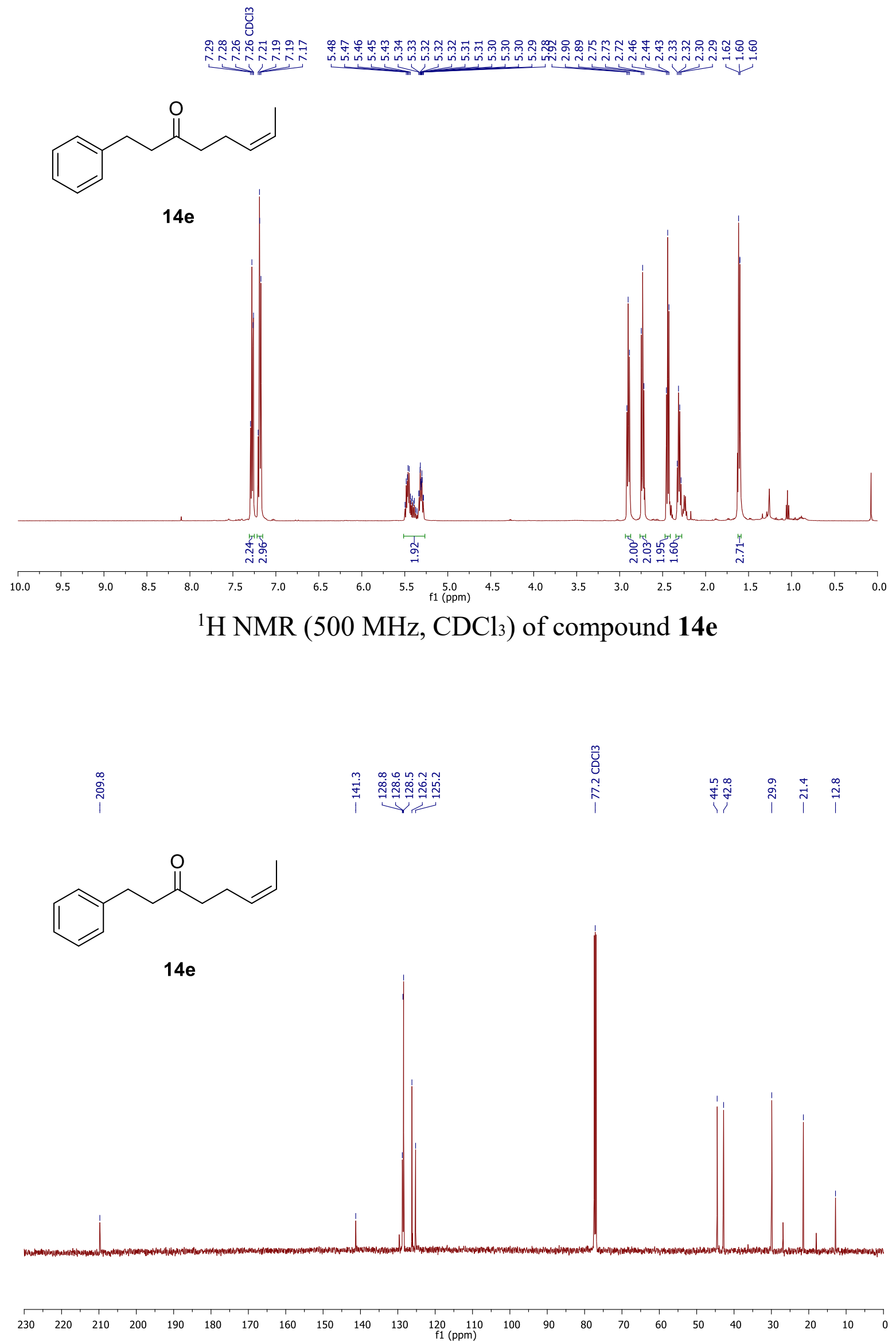

${ }^{13} \mathrm{C}$ NMR (126 MHz, $\mathrm{CDCl}_{3}$ ) of compound $\mathbf{1 4 e}$ 


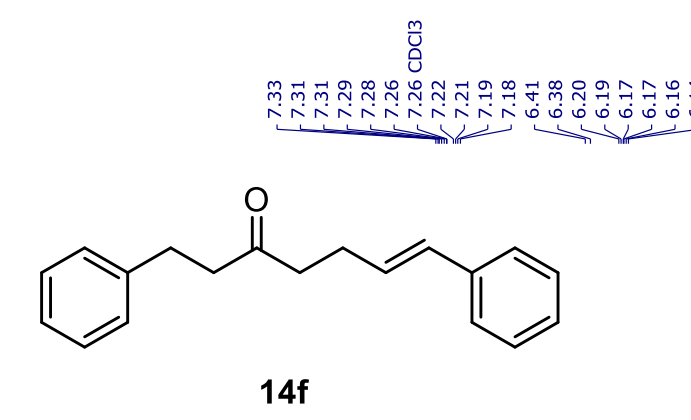

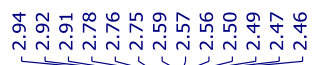

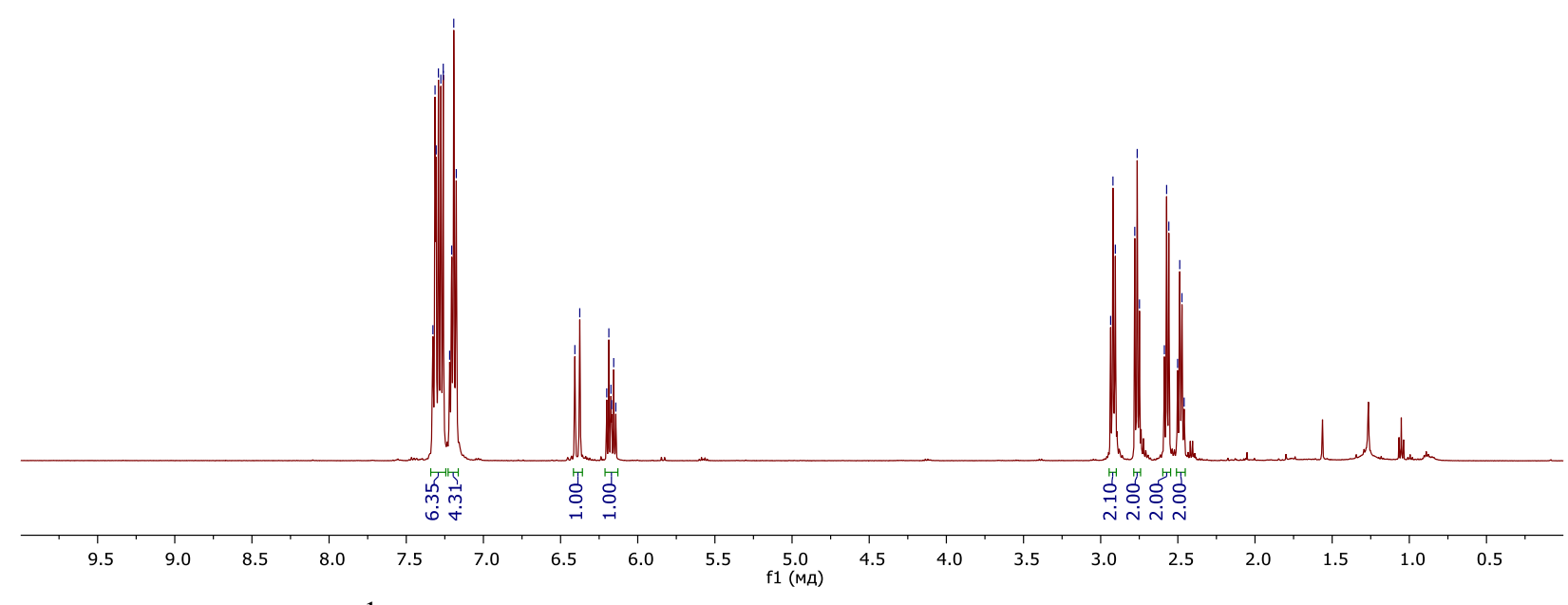

${ }^{1} \mathrm{H}$ NMR (500 MHz, $\mathrm{CDCl}_{3}$ ) of compound $\mathbf{1 4 f}$

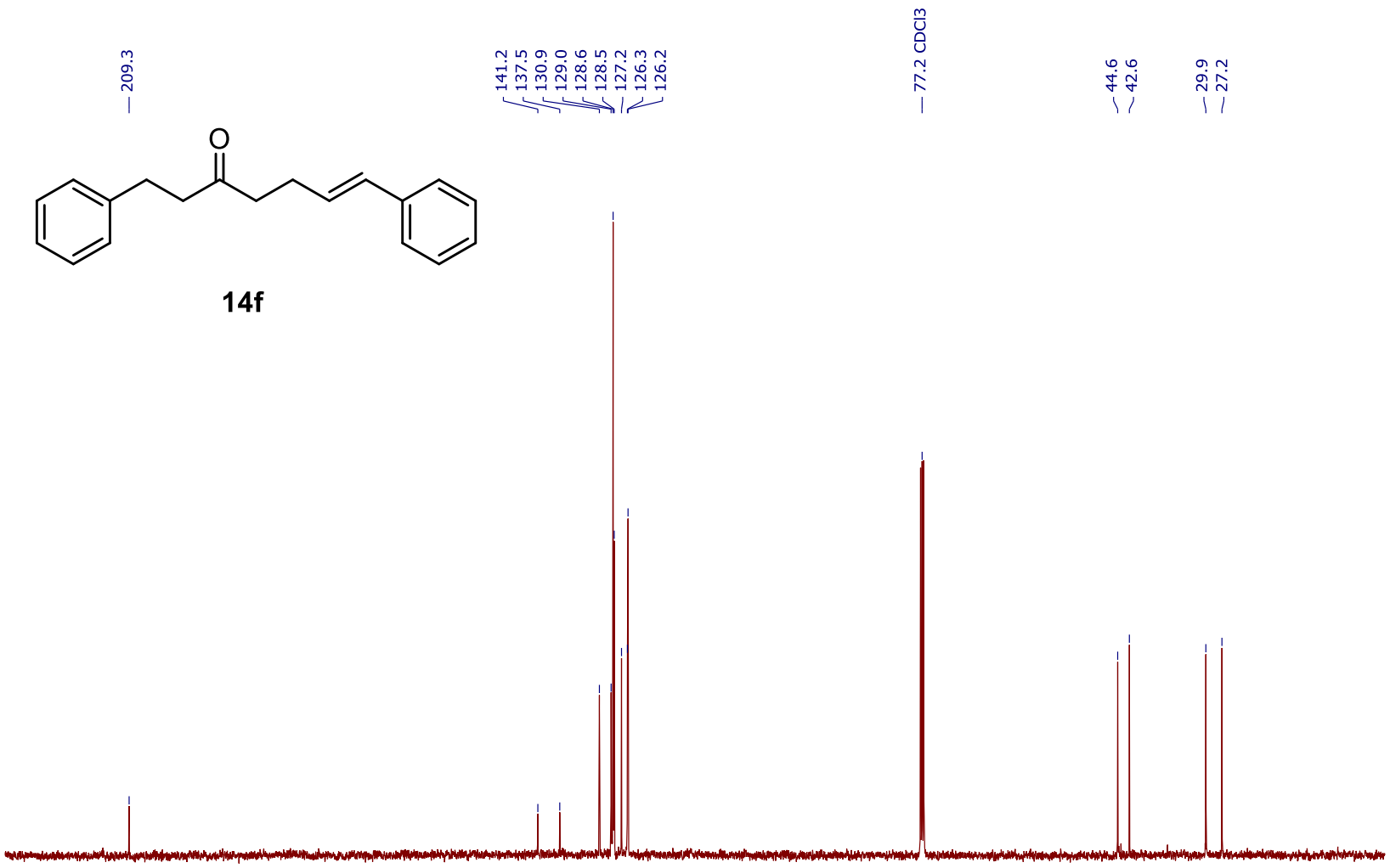

$\begin{array}{llllllllllllllllllllll}220 & 210 & 200 & 190 & 180 & 170 & 160 & 150 & 140 & 130 & \underset{\mathrm{f} 1(\mathrm{MA})}{110} & 100 & 90 & 80 & 70 & 60 & 50 & 40 & 30 & 20 & 10 & \end{array}$

${ }^{13} \mathrm{C} \mathrm{NMR}\left(126 \mathrm{MHz}, \mathrm{CDCl}_{3}\right)$ of compound $\mathbf{1 4 f}$ 

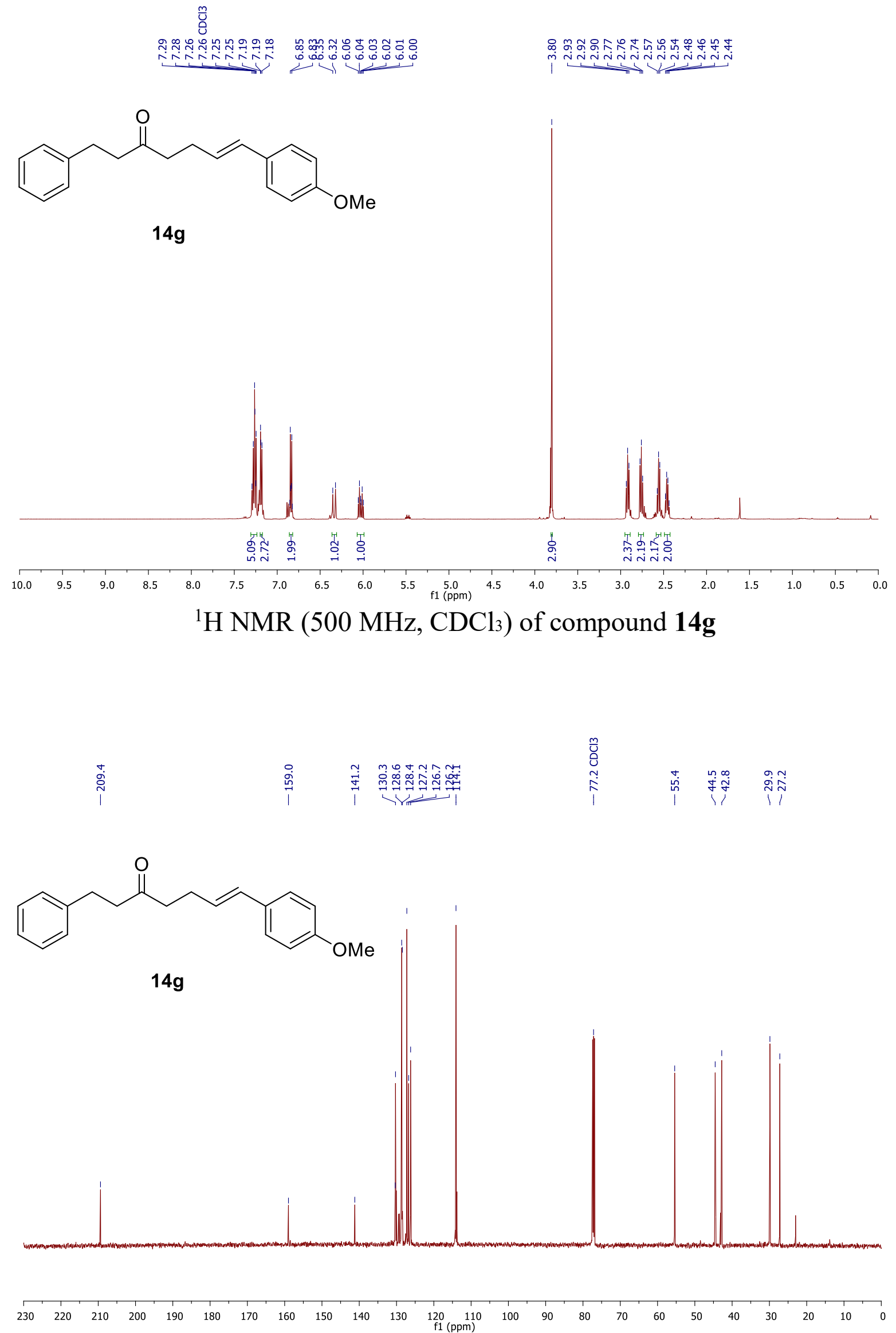

${ }^{13} \mathrm{C}$ NMR (126 MHz, $\mathrm{CDCl}_{3}$ ) of compound $\mathbf{1 4 g}$ 

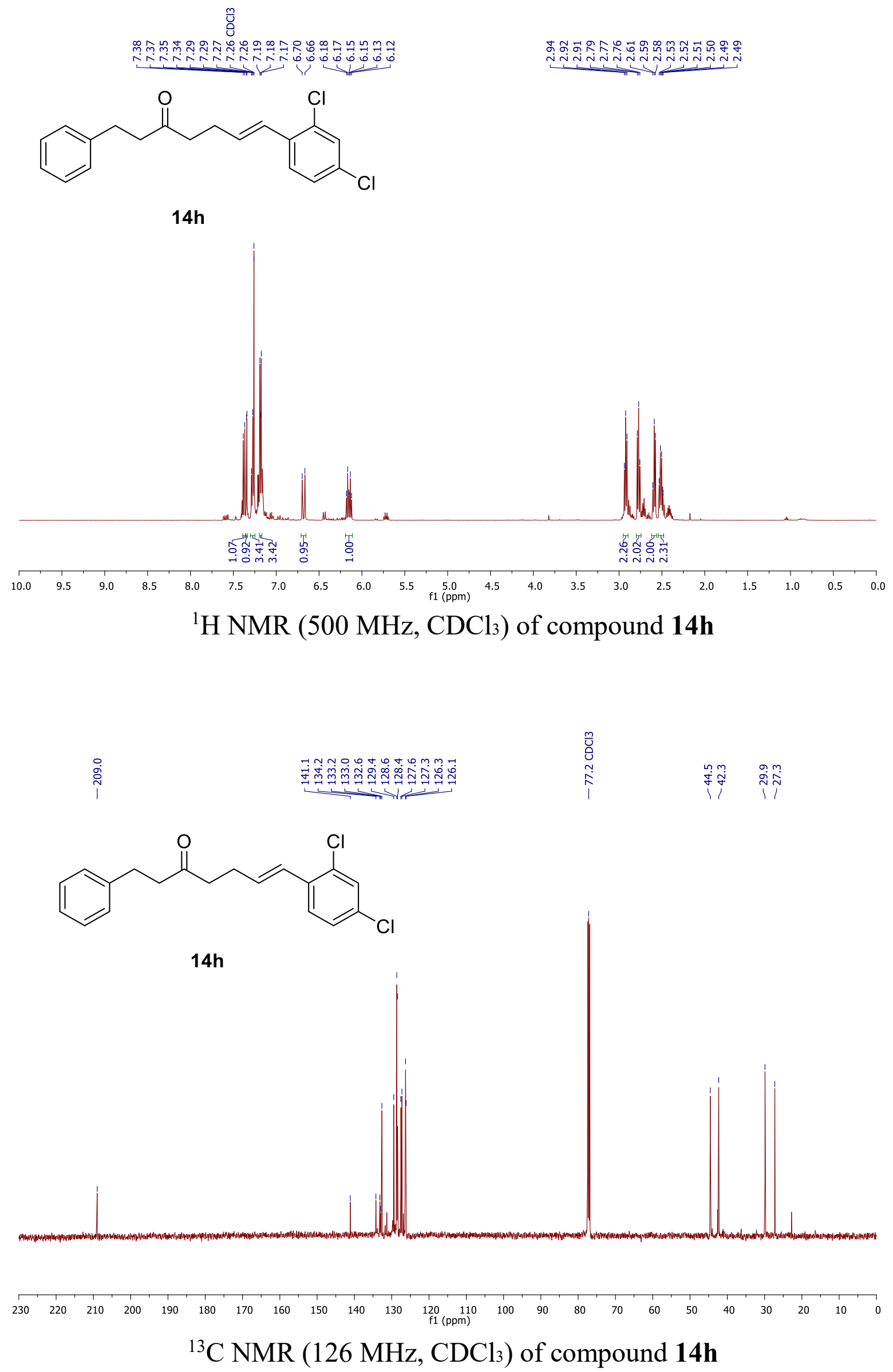


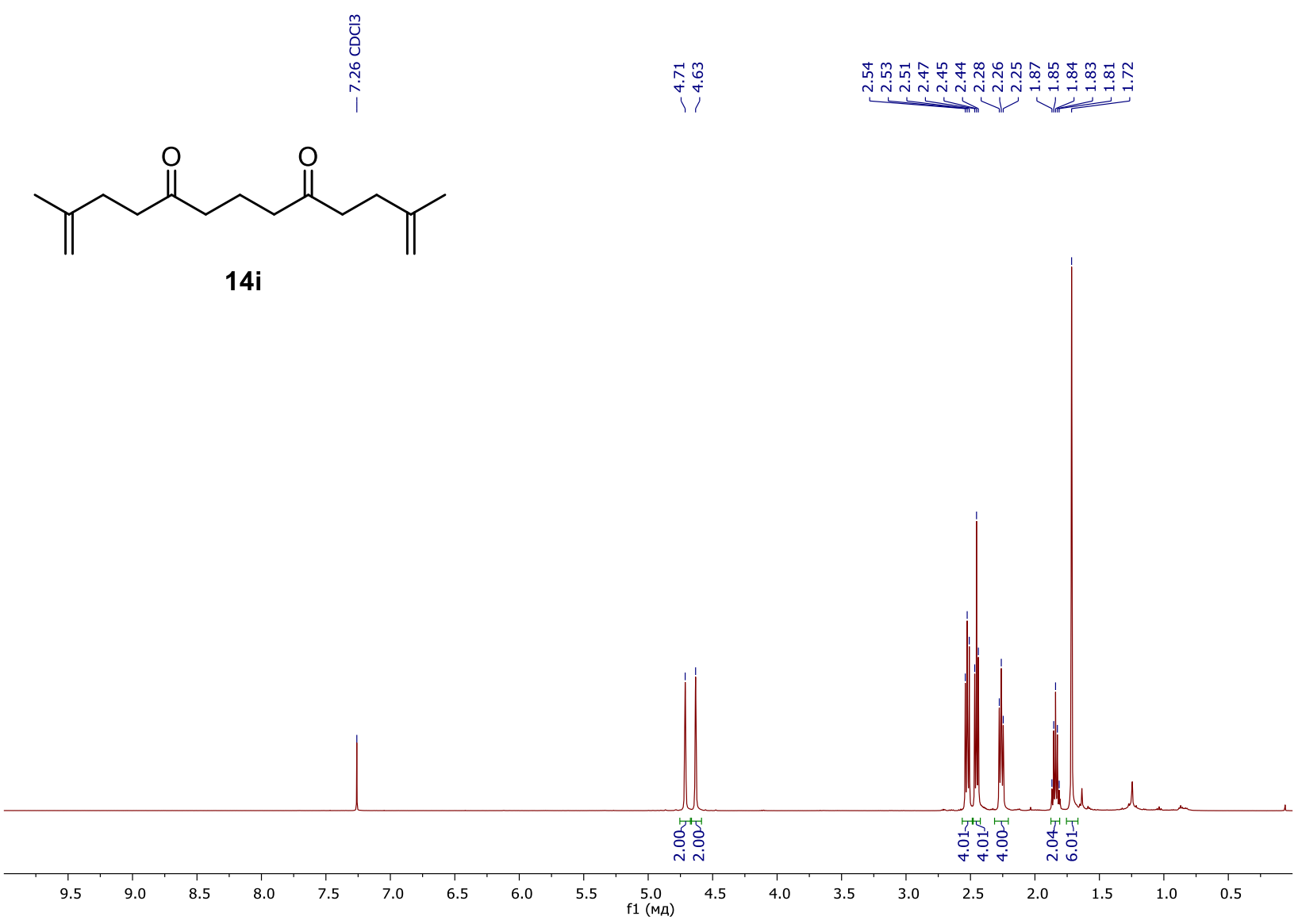

${ }^{1} \mathrm{H}$ NMR (500 MHz, $\mathrm{CDCl}_{3}$ ) of compound 14i

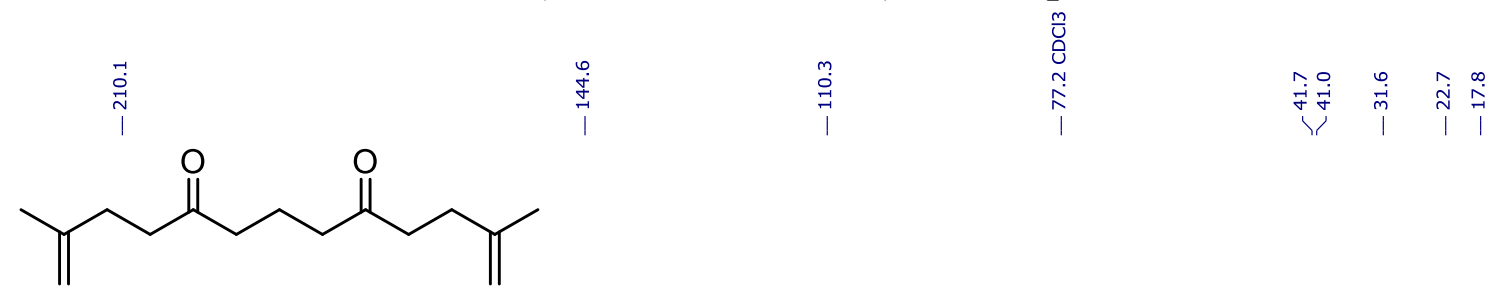

$14 i$

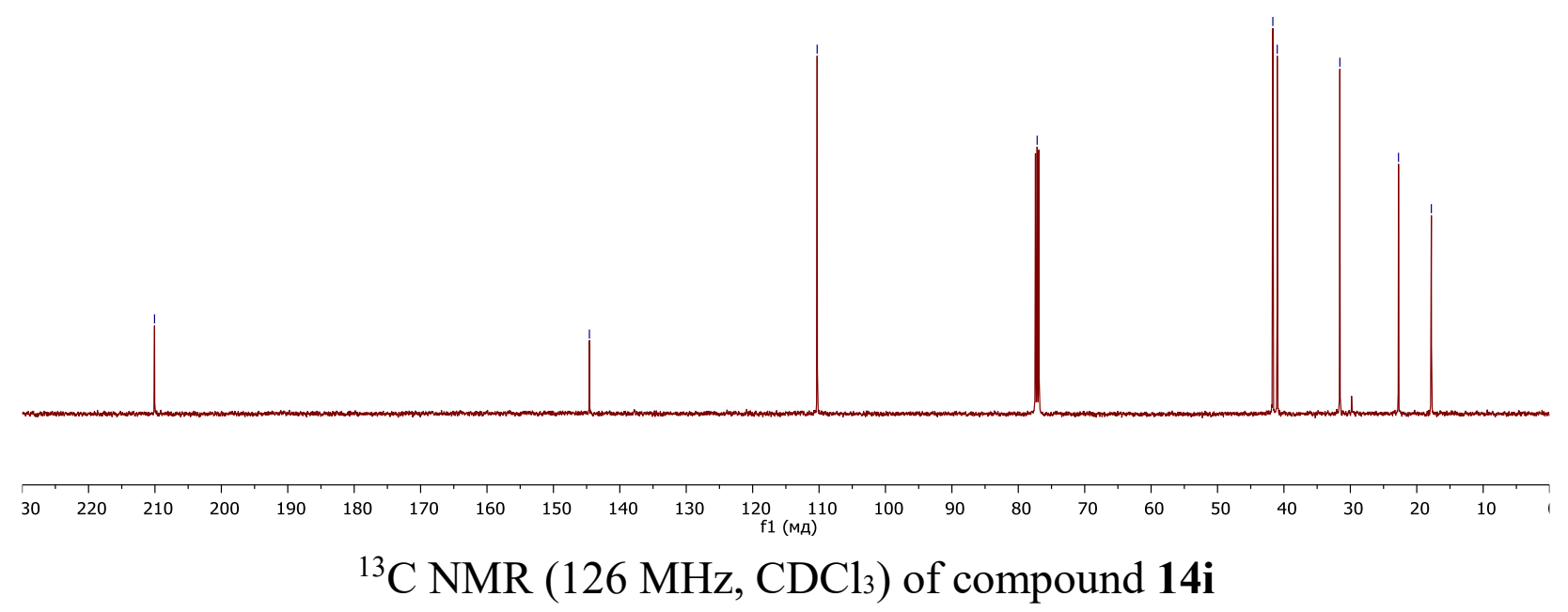




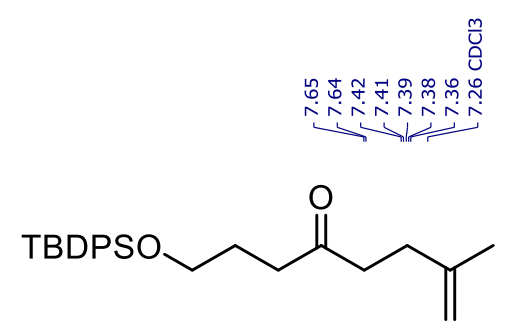

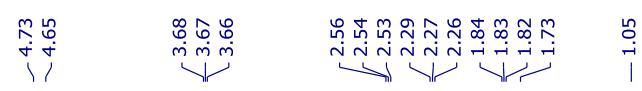

14j

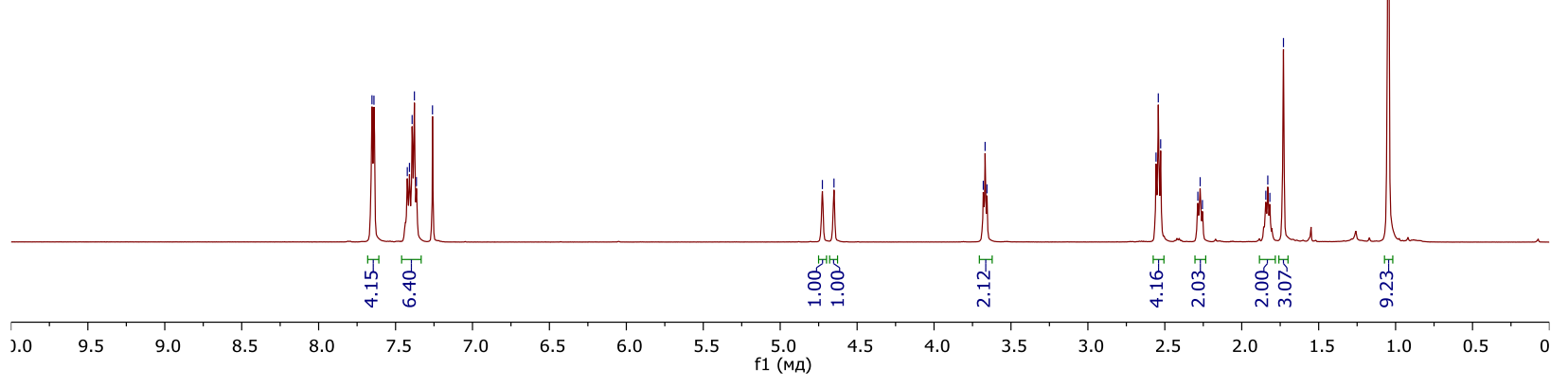

${ }^{1} \mathrm{H} \mathrm{NMR}\left(500 \mathrm{MHz}, \mathrm{CDCl}_{3}\right)$ of compound $\mathbf{1 4} \mathbf{j}$

$\stackrel{\substack{n \\ \stackrel{n}{1}}}{1}$

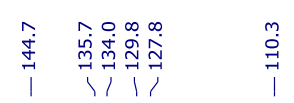

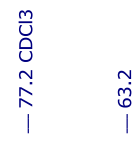

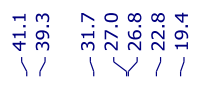

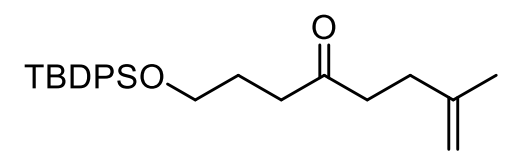

14j

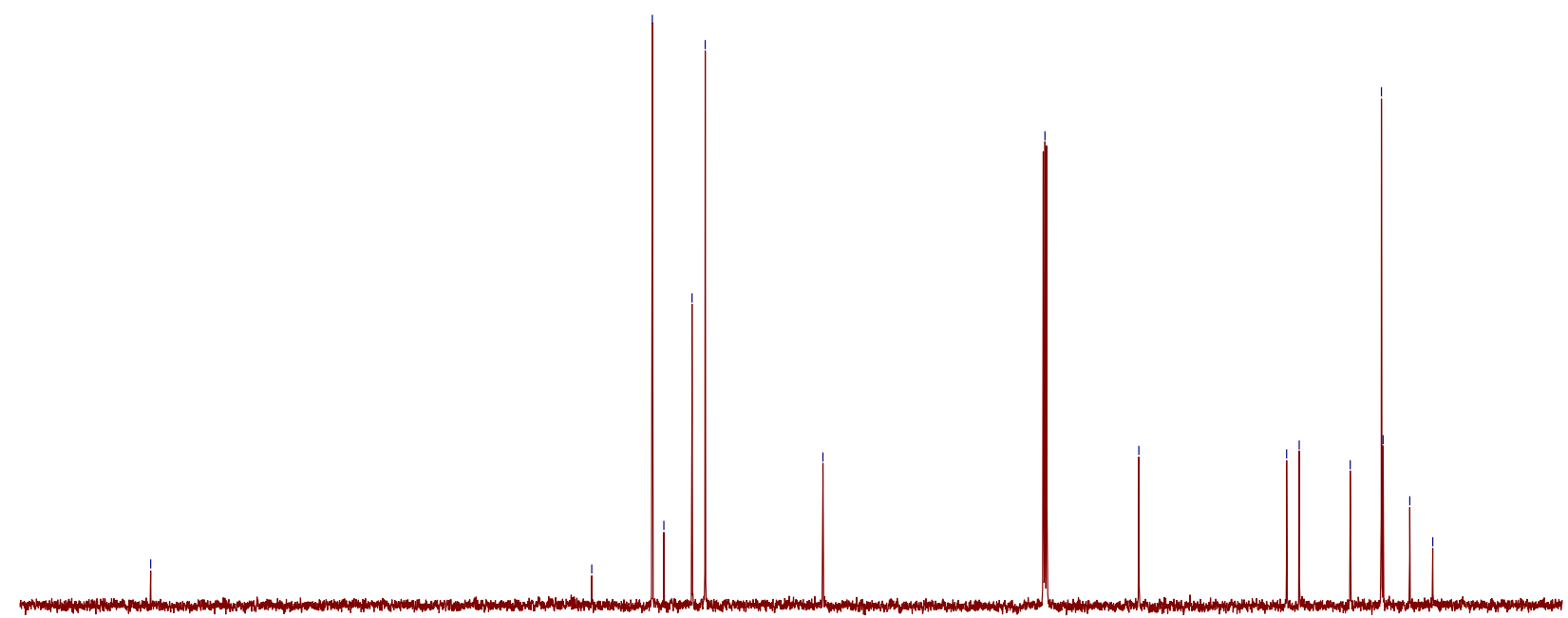

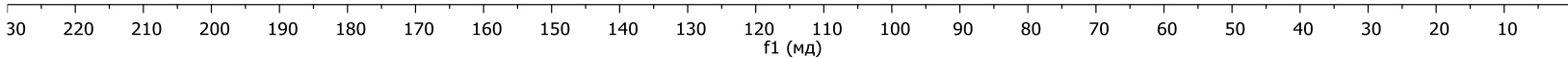

${ }^{13} \mathrm{C}$ NMR (126 MHz, $\left.\mathrm{CDCl}_{3}\right)$ of compound $\mathbf{1 4} \mathbf{j}$ 


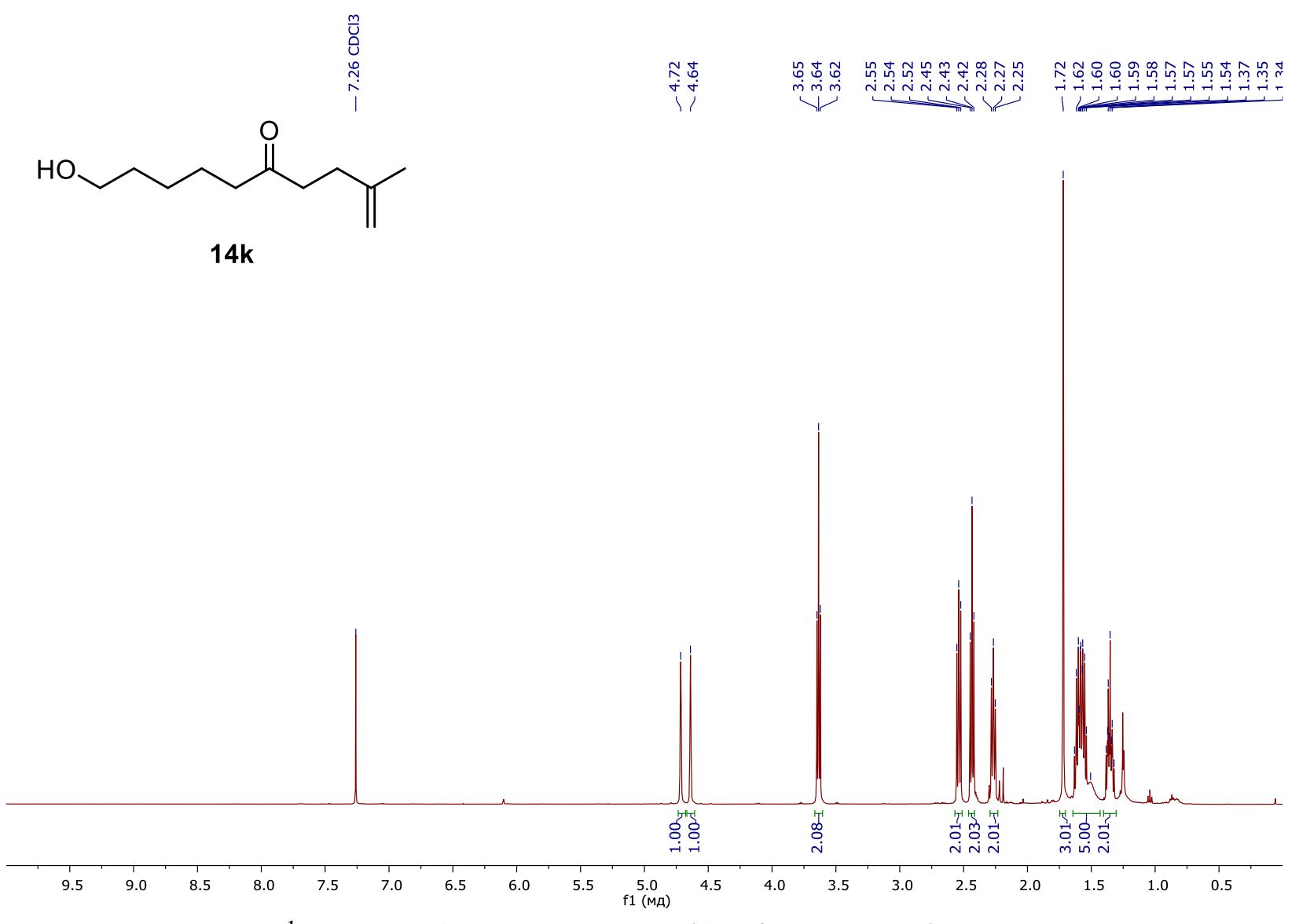

${ }^{1} \mathrm{H}$ NMR (500 MHz, $\mathrm{CDCl}_{3}$ ) of compound 14k
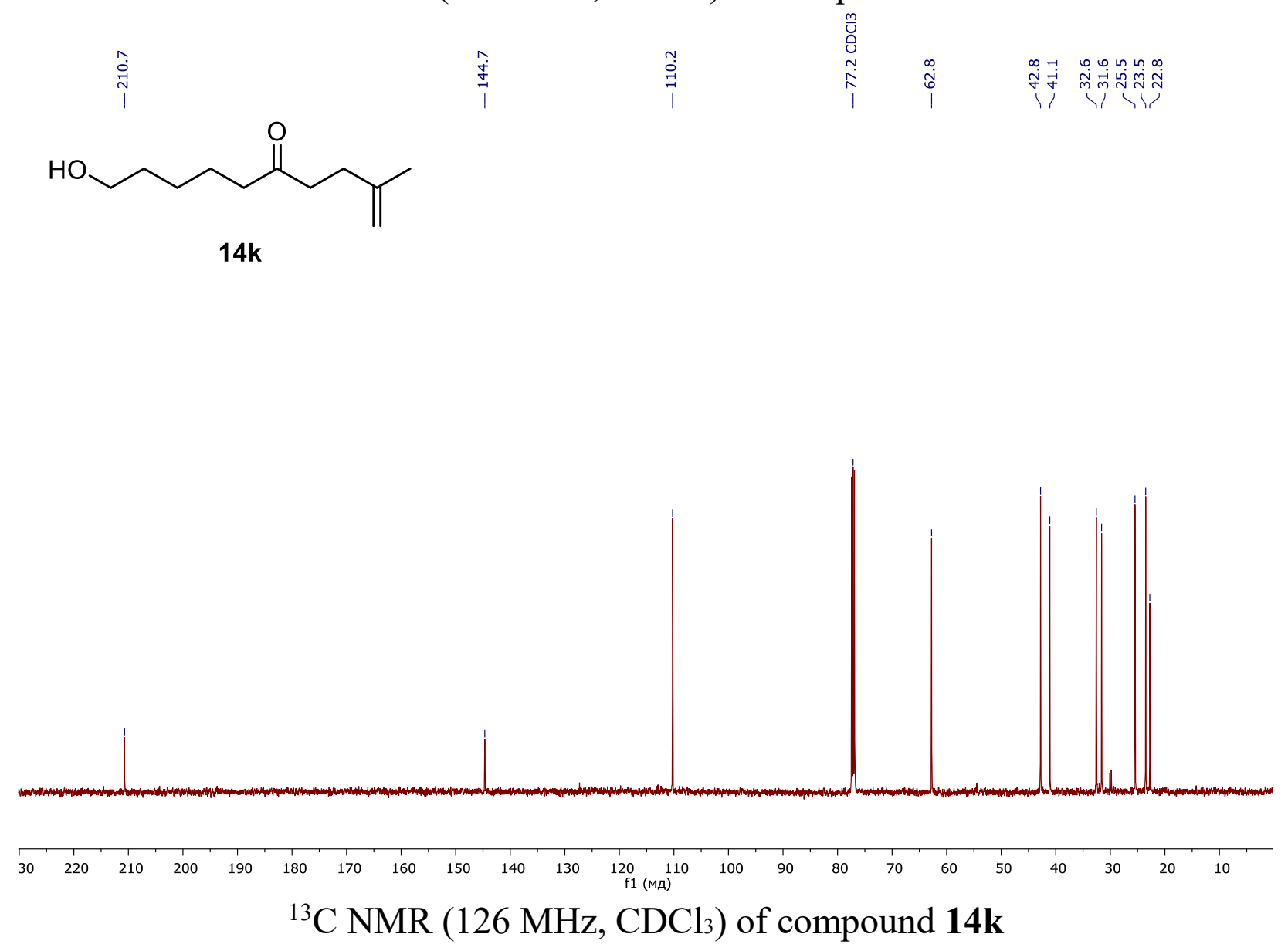
<smiles>[C+]=NI</smiles>

$14 \mid$

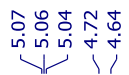

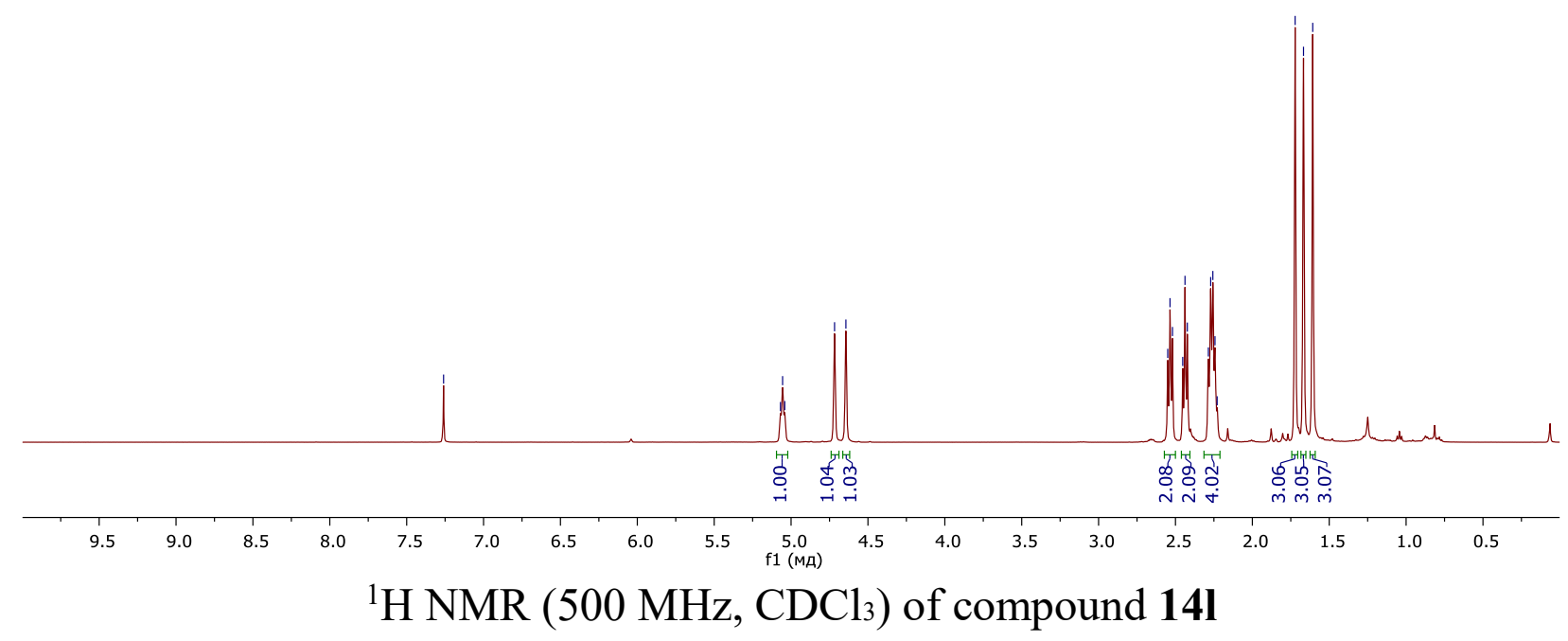<smiles>C=C(C)CCC(=O)CCC=C(C)C</smiles>

14 I

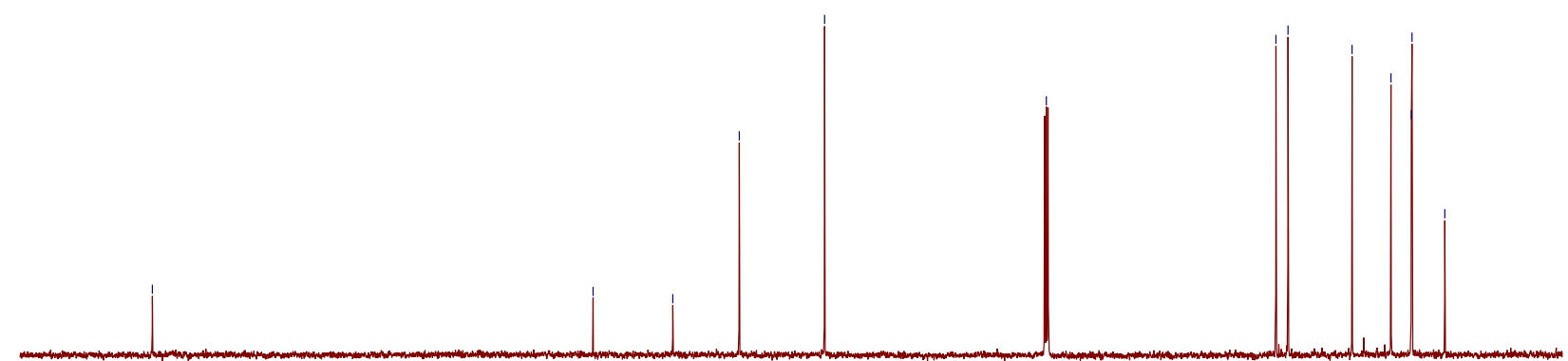

$\begin{array}{llllllllllllllllllllllll}30 & 220 & 210 & 200 & 190 & 180 & 170 & 160 & 150 & 140 & 130 & 120 & 110 & 100 & 90 & 80 & 70 & 60 & 50 & 40 & 30 & 20 & 10\end{array}$

${ }^{13} \mathrm{C} \mathrm{NMR}\left(126 \mathrm{MHz}, \mathrm{CDCl}_{3}\right)$ of compound 141 


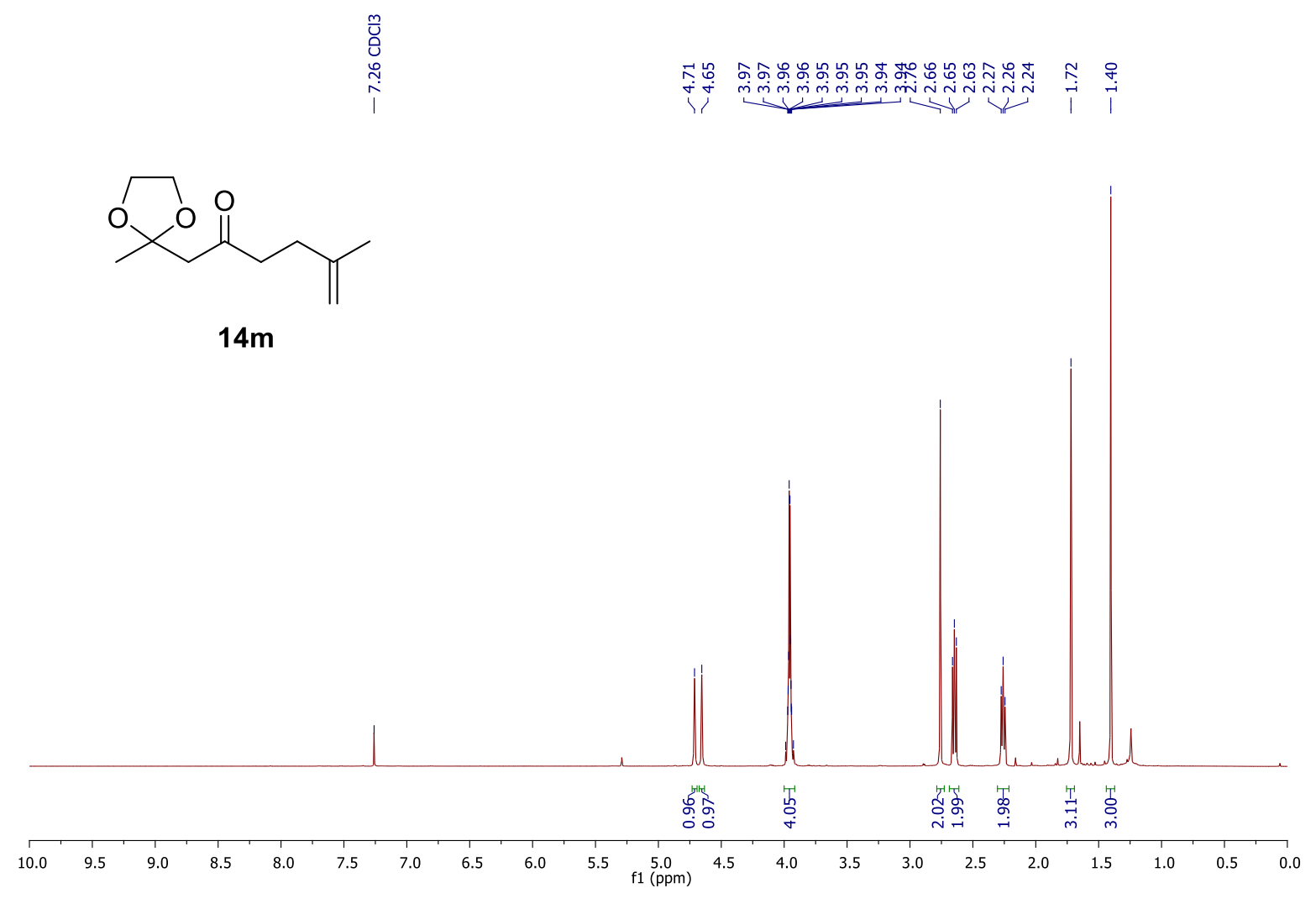

${ }^{1} \mathrm{H}$ NMR $\left(500 \mathrm{MHz}, \mathrm{CDCl}_{3}\right)$ of compound $\mathbf{1 4 m}$

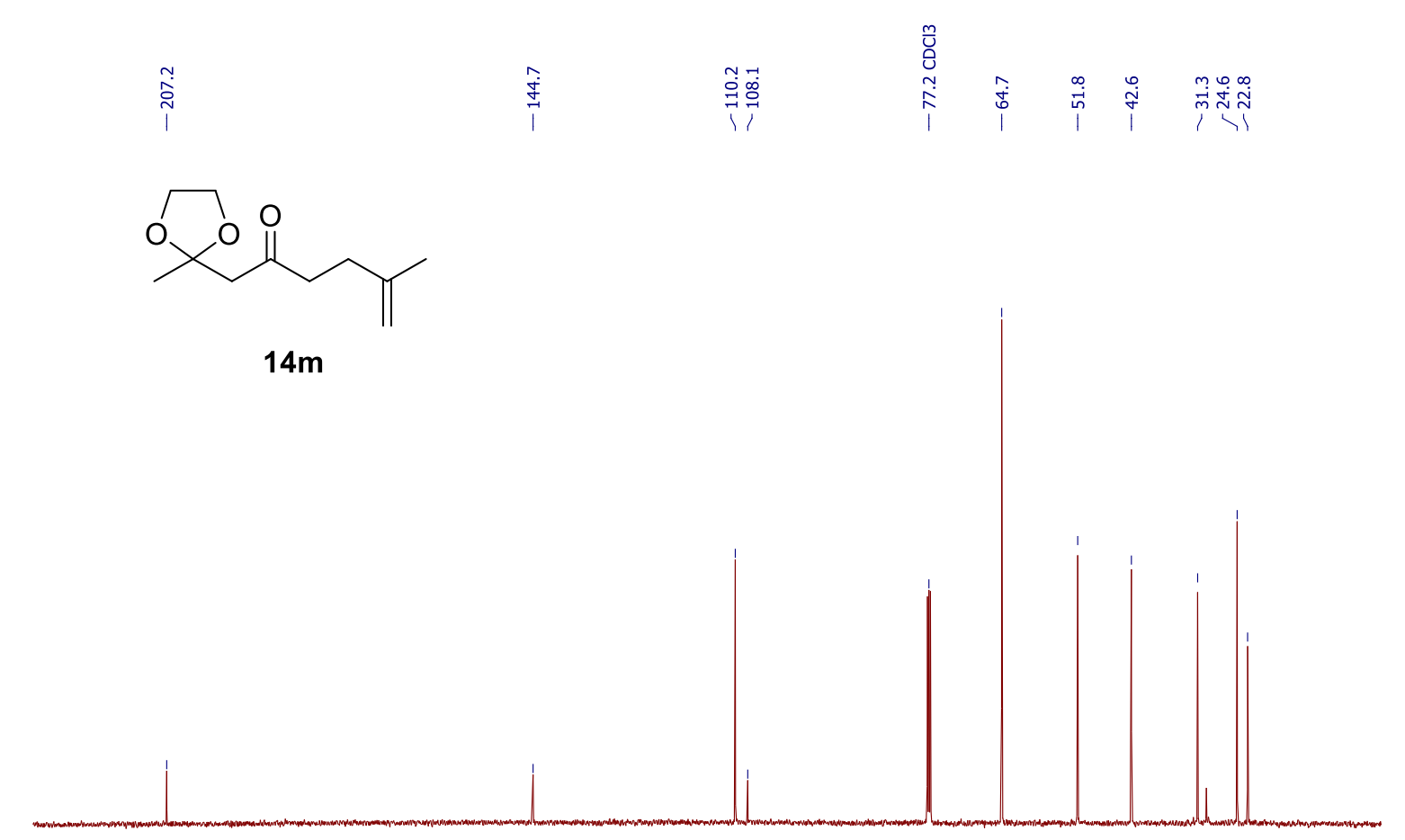

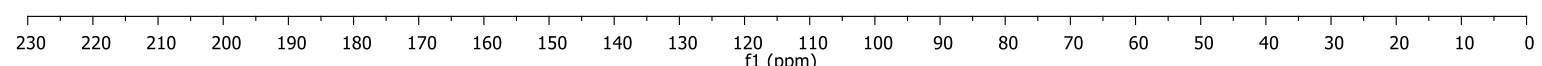

${ }^{13} \mathrm{C}$ NMR (126 MHz, $\left.\mathrm{CDCl}_{3}\right)$ of compound $\mathbf{1 4 m}$ 
<smiles>C=C(C)CCC(=O)CC(OCC)OCC</smiles>

$14 n$

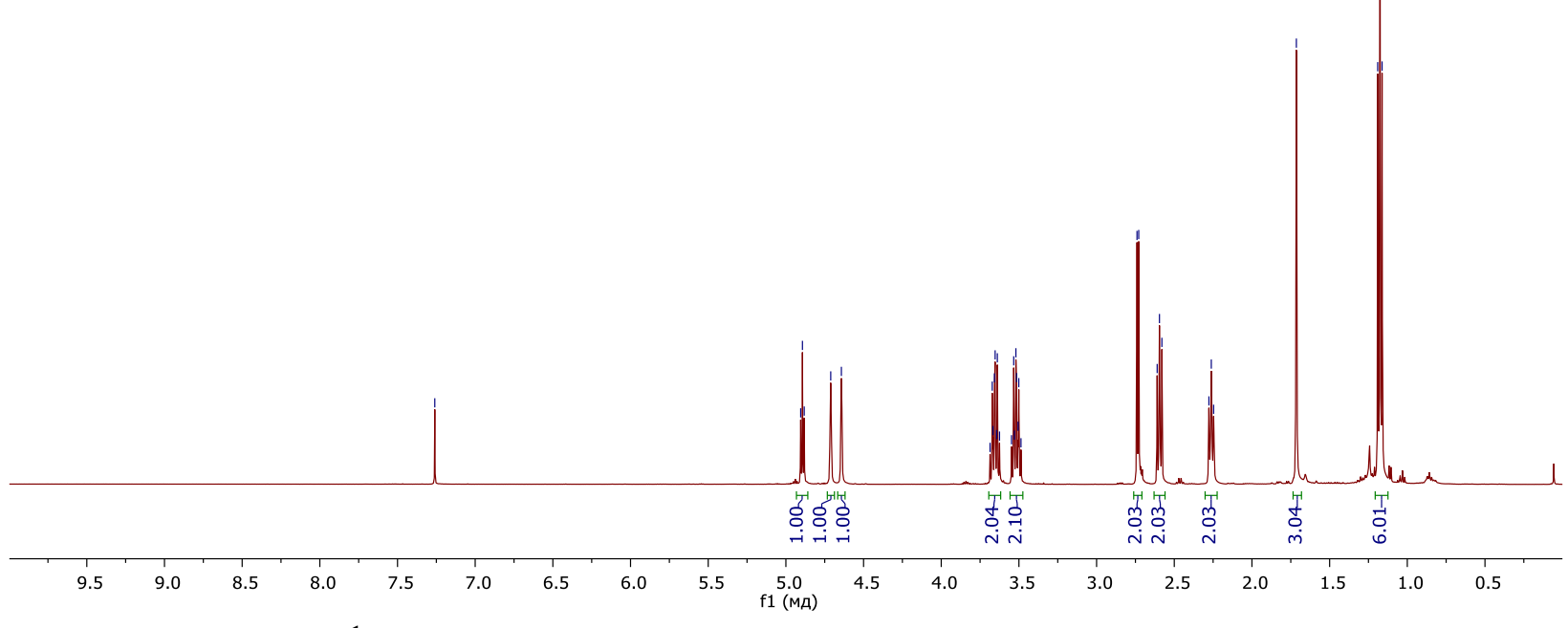

${ }^{1} \mathrm{H} \mathrm{NMR}\left(500 \mathrm{MHz}, \mathrm{CDCl}_{3}\right)$ of compound 14n
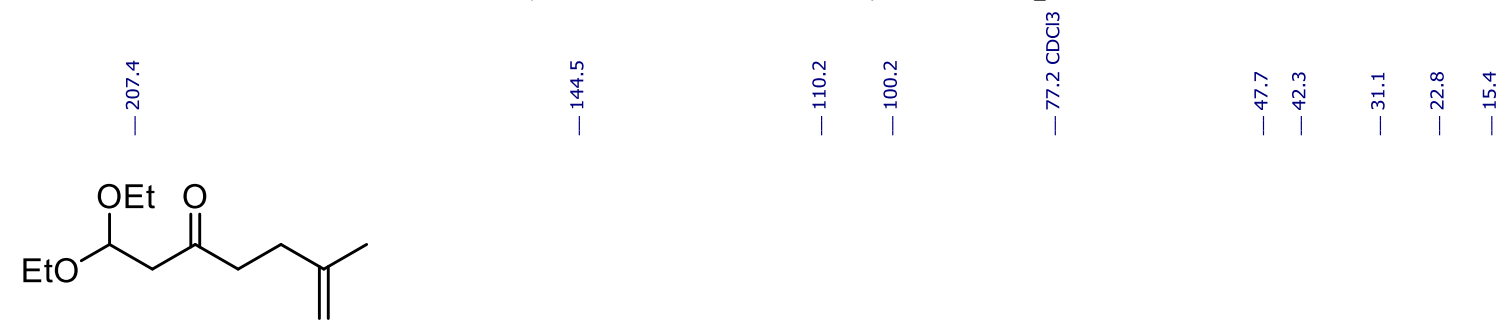

$14 n$

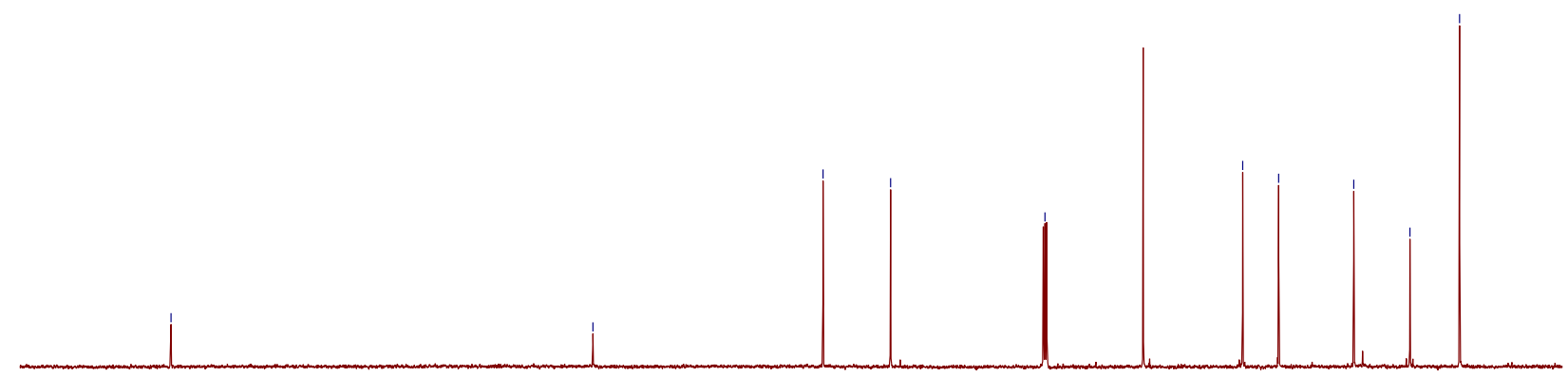

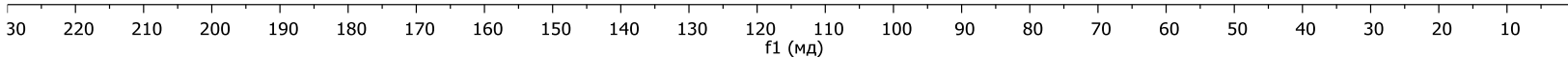

${ }^{13} \mathrm{C}$ NMR (126 MHz, $\left.\mathrm{CDCl}_{3}\right)$ of compound $\mathbf{1 4 n}$ 
<smiles>C=C(C)CCC(=O)C(C)[OH2+]</smiles>

140

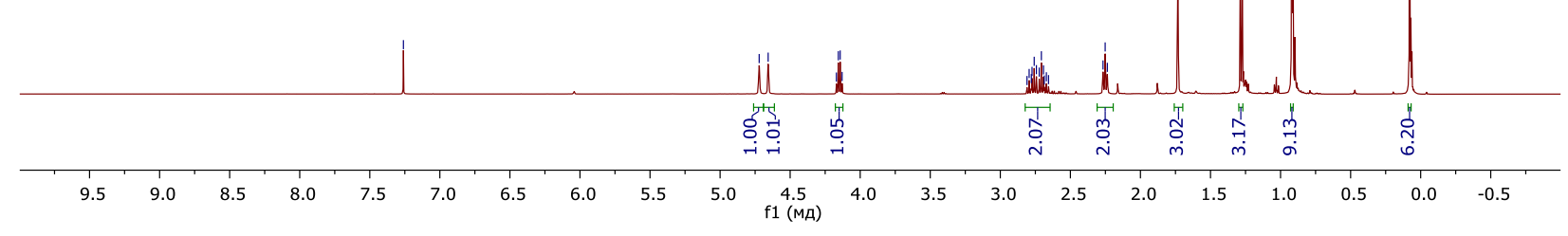

${ }^{1} \mathrm{H}$ NMR (500 MHz, $\mathrm{CDCl}_{3}$ ) of compound 140

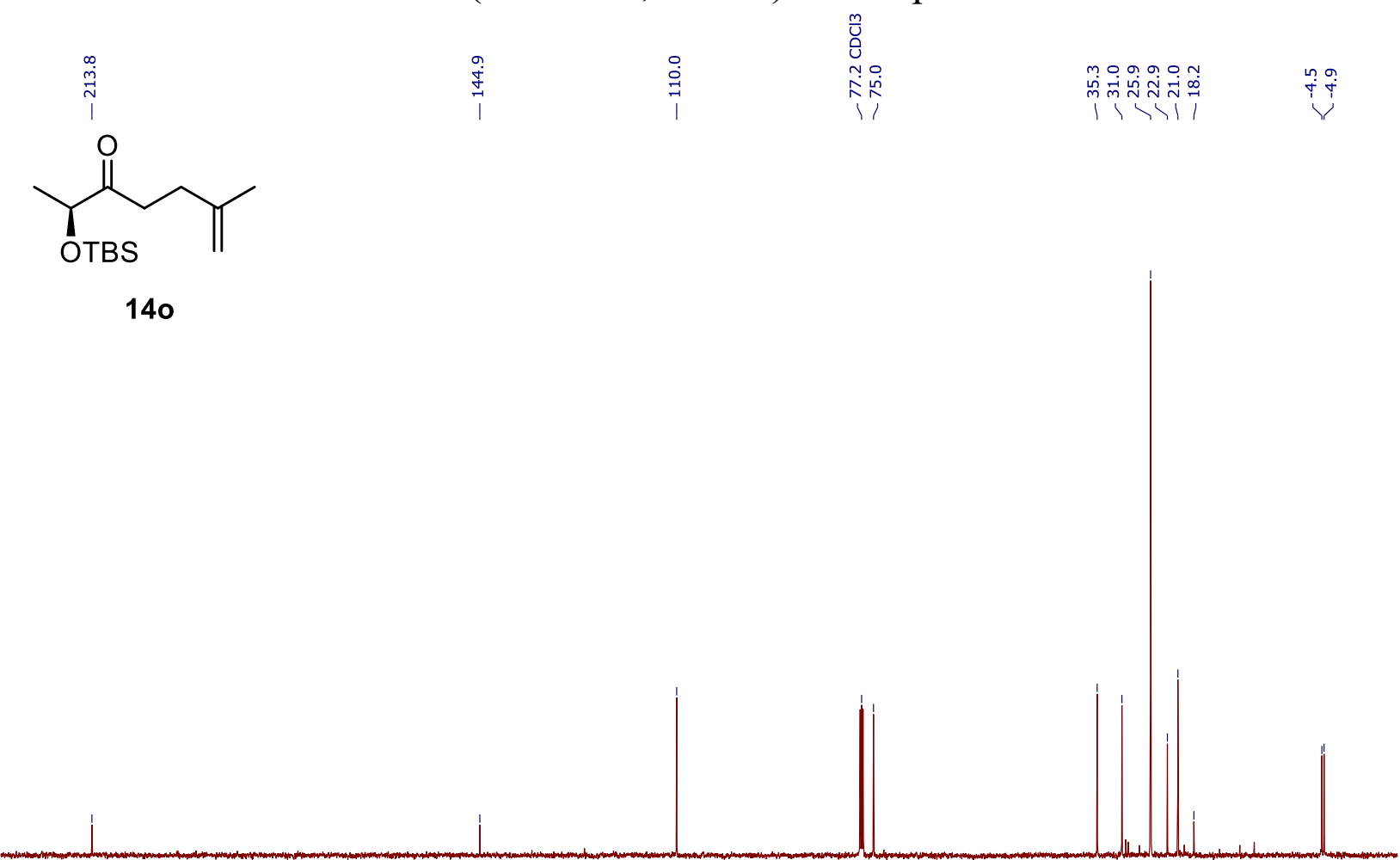

\begin{tabular}{lllllllllllllllllllllllllllll}
\hline 30 & 220 & 210 & 200 & 190 & 180 & 170 & 160 & 150 & 140 & 130 & 120 & $\underset{\mathrm{f} 1(\mathrm{MA})}{110}$ & 100 & 90 & 80 & 70 & 60 & 50 & 40 & 30 & 20 & 10 & 0 & -10
\end{tabular}

${ }^{13} \mathrm{C} \mathrm{NMR}\left(126 \mathrm{MHz}, \mathrm{CDCl}_{3}\right)$ of compound 140 
<smiles>C=C(C)CCC(=O)[C@H](C)COS(=O)(=O)c1ccccc1</smiles>

$14 p$

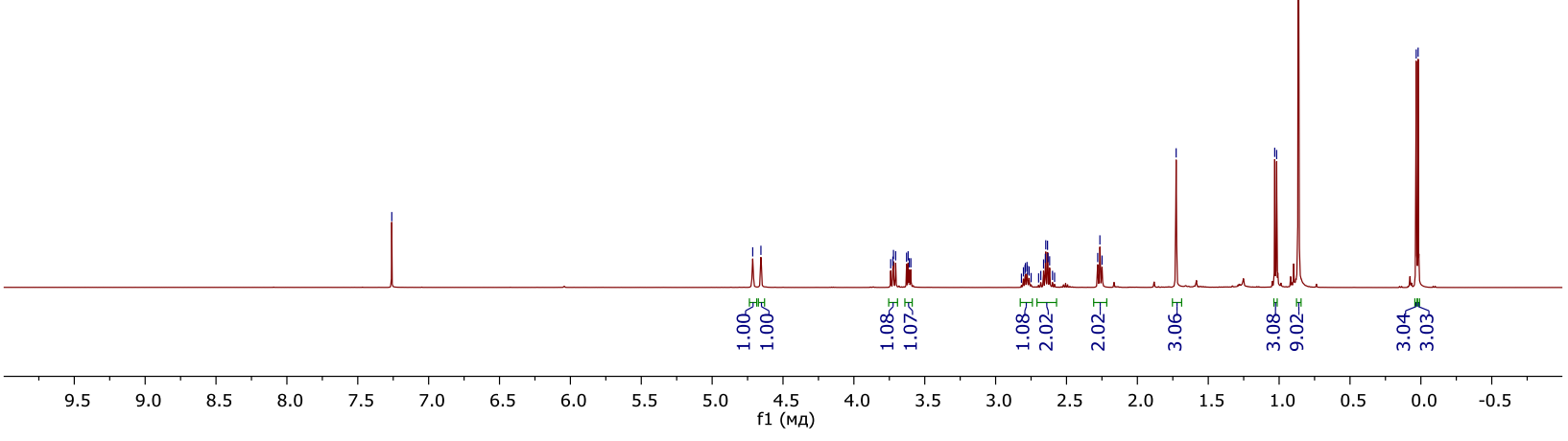

${ }^{1} \mathrm{H}$ NMR $\left(500 \mathrm{MHz}, \mathrm{CDCl}_{3}\right)$ of compound $\mathbf{1 4 p}$

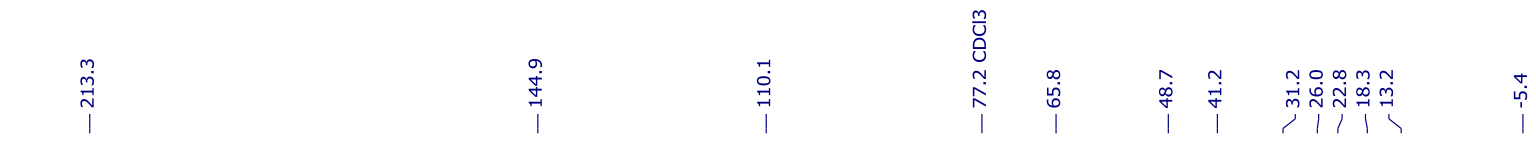<smiles></smiles>

$14 p$

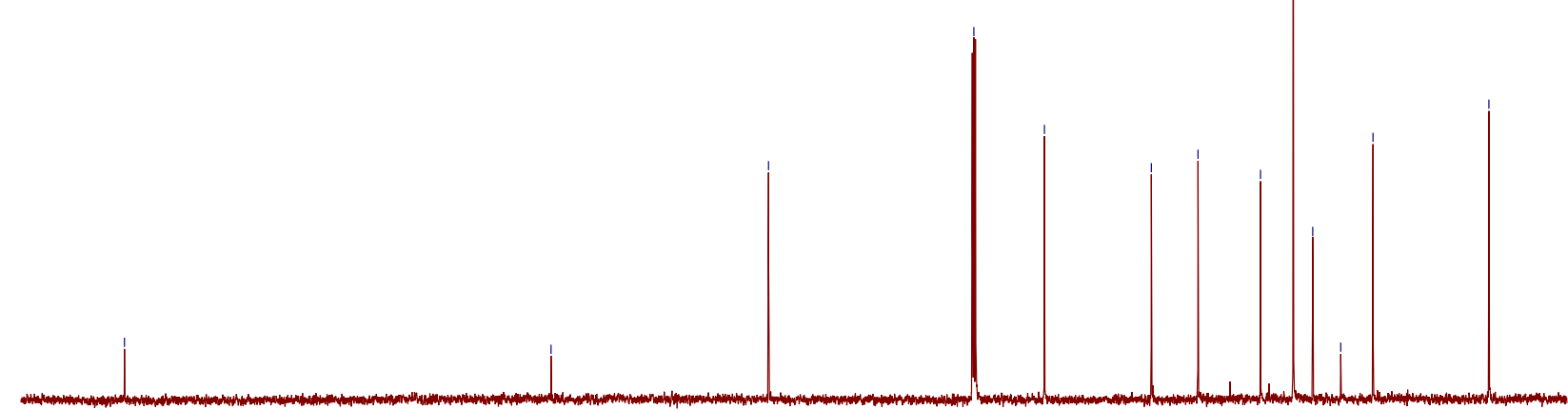

$\begin{array}{llllllllllllllllllllllllll}30 & 220 & 210 & 200 & 190 & 180 & 170 & 160 & 150 & 140 & 130 & 120 & \underset{\mathrm{f} 1(\mathrm{MA})}{110} & 90 & 80 & 70 & 60 & 50 & 40 & 30 & 20 & 10 & 0 & -10\end{array}$

${ }^{13} \mathrm{C}$ NMR (126 MHz, $\mathrm{CDCl}_{3}$ ) of compound 14p 

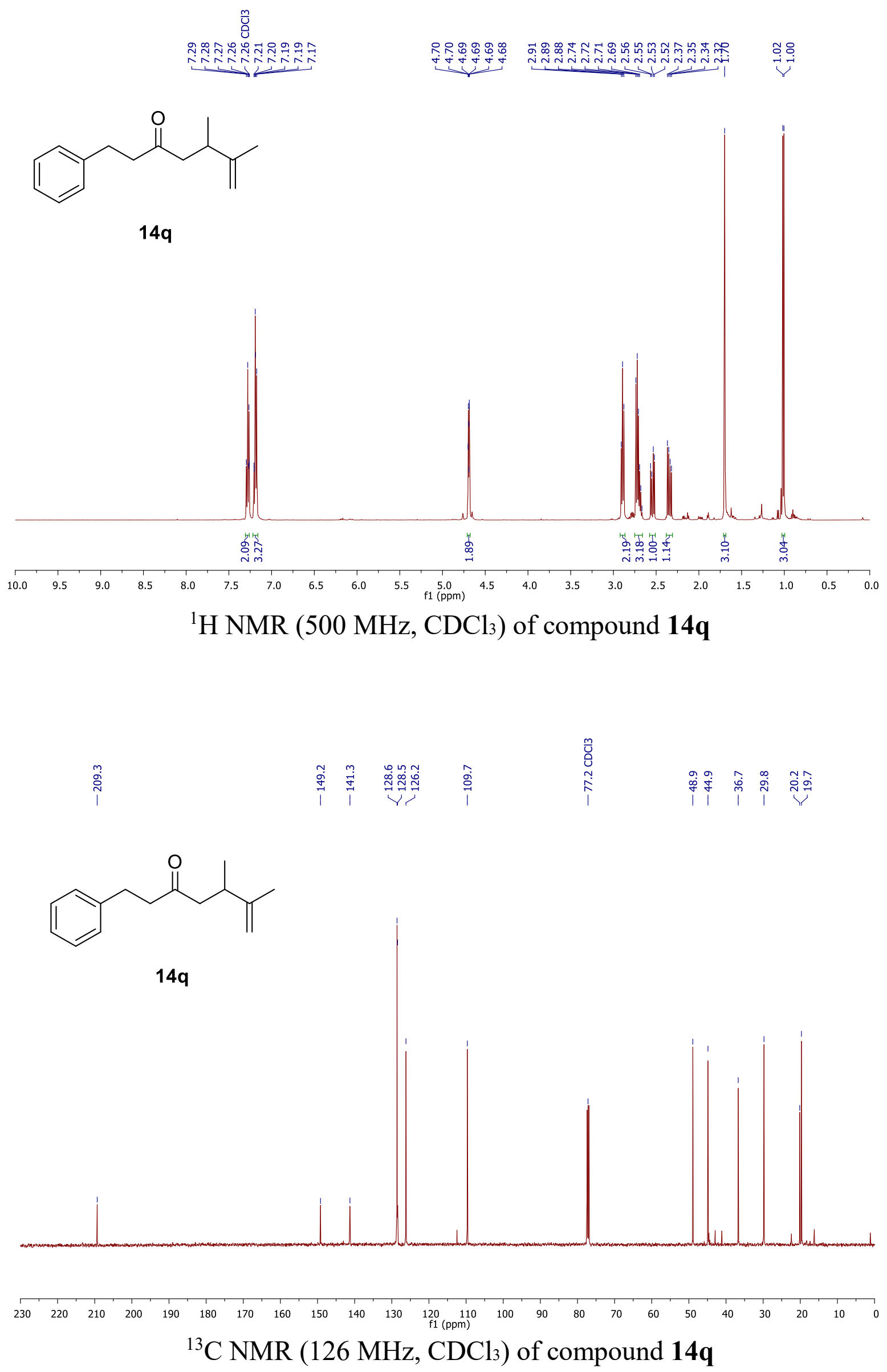

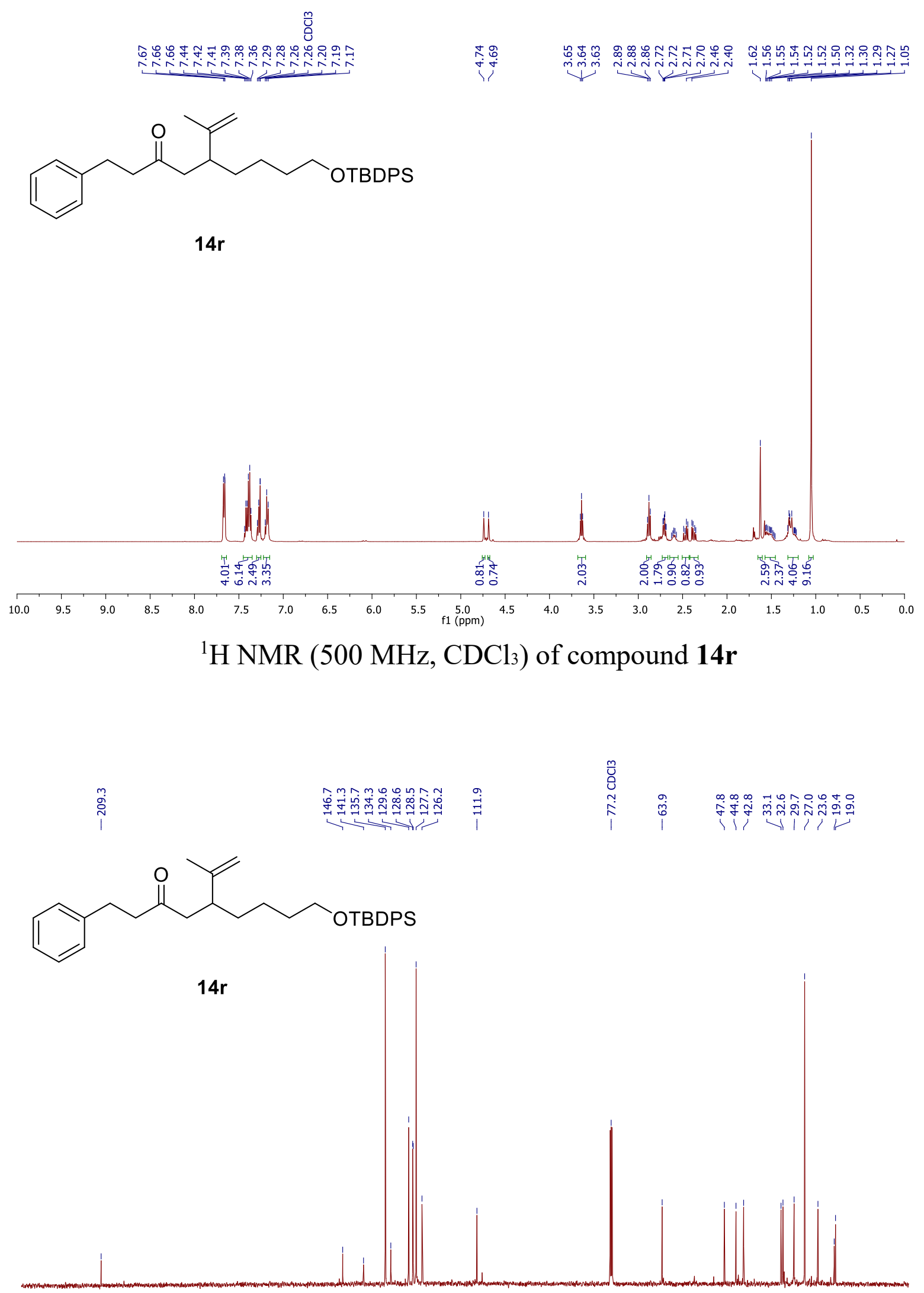

\begin{tabular}{rlllllllllllllllllllllllllll}
\hline 230 & 220 & 210 & 200 & 190 & 180 & 170 & 160 & 150 & 140 & 130 & 120 & 110 & 100 & 90 & 80 & 70 & 60 & 50 & 40 & 30 & 20 & 10 & 0
\end{tabular}

${ }^{13} \mathrm{C}$ NMR (126 MHz, $\left.\mathrm{CDCl}_{3}\right)$ of compound $\mathbf{1 4 r}$ 
<smiles>C=C(C)C(CC(=O)CCc1ccccc1)CC(CC)O[Na]</smiles>

$14 s$

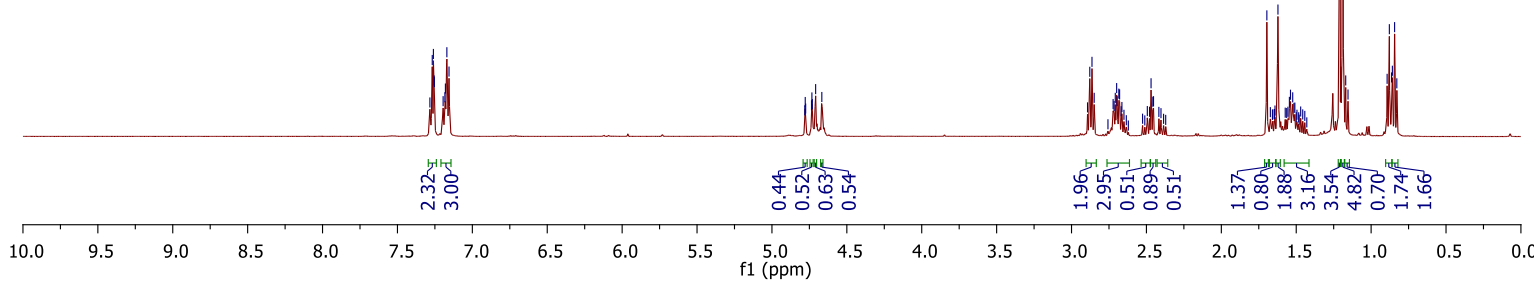

${ }^{1} \mathrm{H}$ NMR (500 MHz, $\mathrm{CDCl}_{3}$ ) of compound $14 \mathrm{~s}$

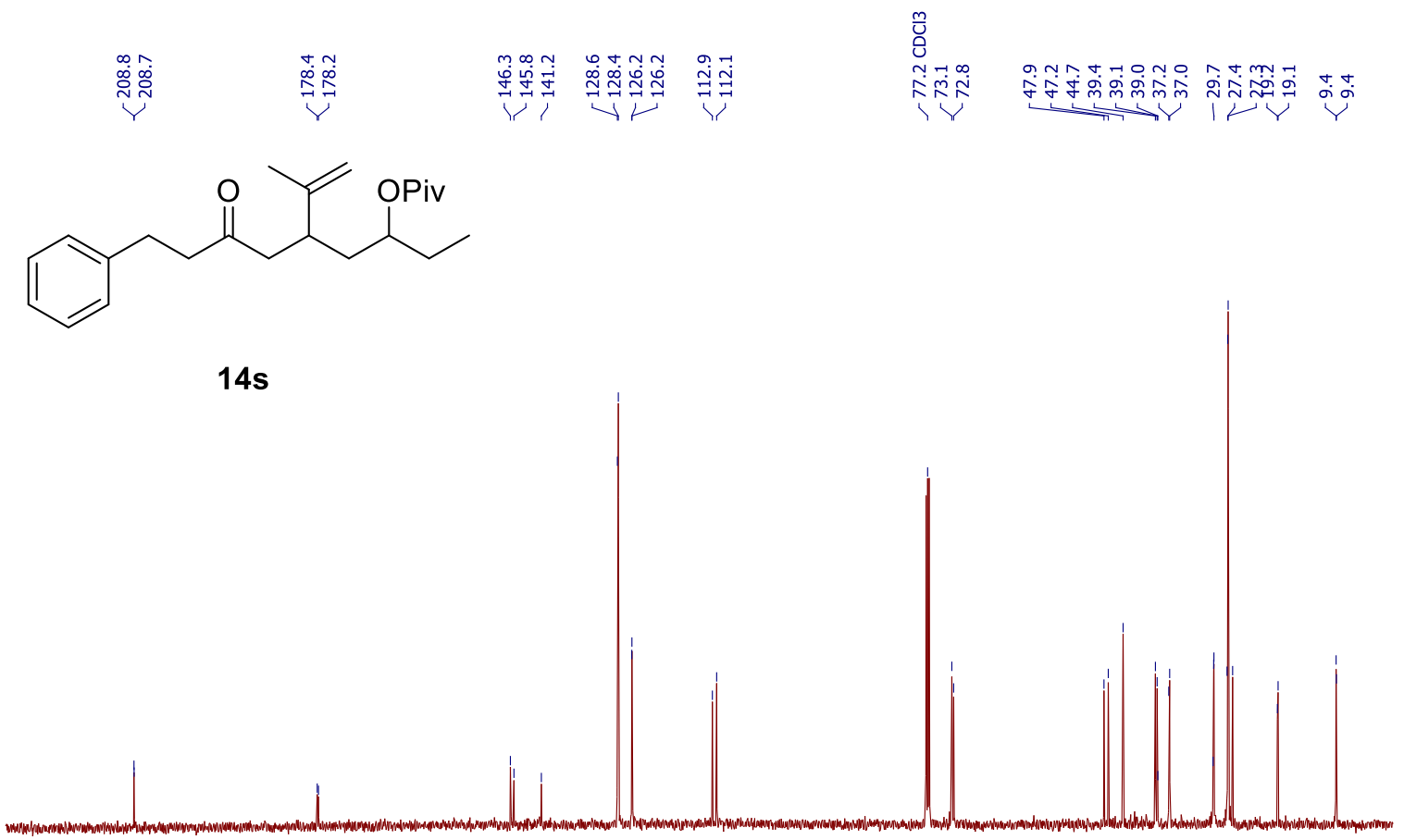

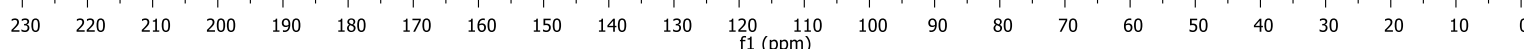

${ }^{13} \mathrm{C}$ NMR (126 MHz, $\left.\mathrm{CDCl}_{3}\right)$ of compound $\mathbf{1 4 s}$ 


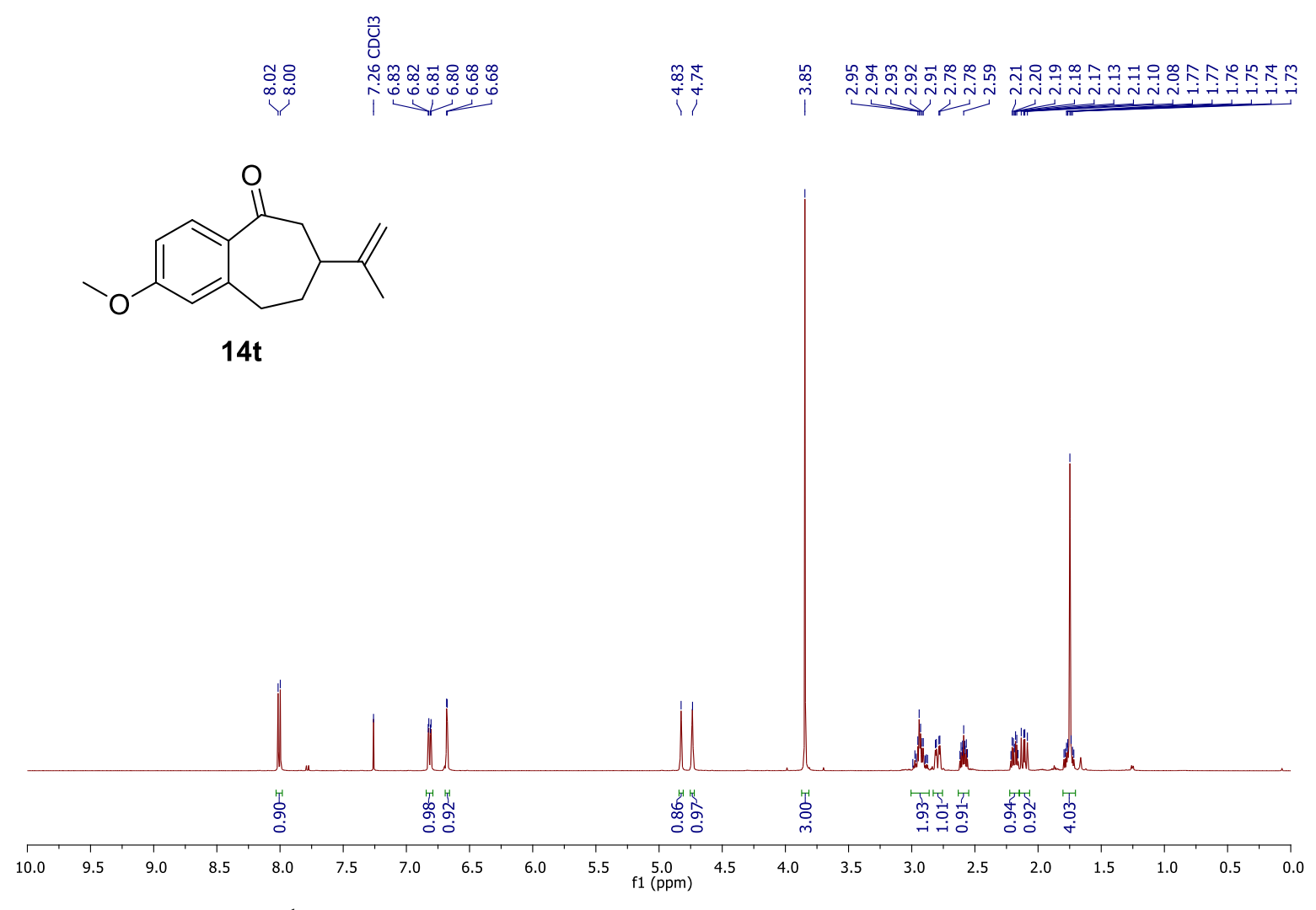

${ }^{1} \mathrm{H}$ NMR $\left(500 \mathrm{MHz}, \mathrm{CDCl}_{3}\right)$ of compound $\mathbf{1 4 t}$

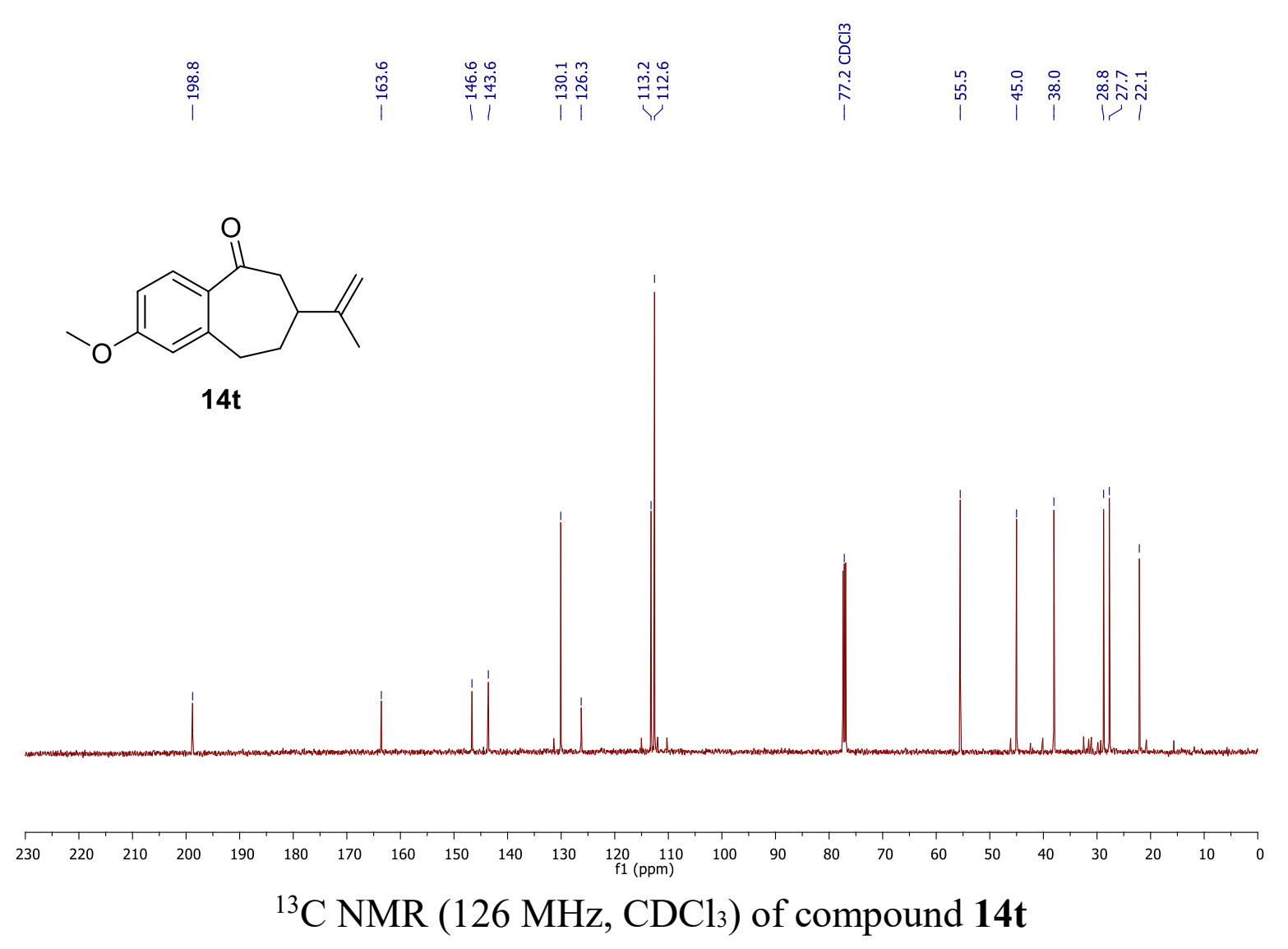



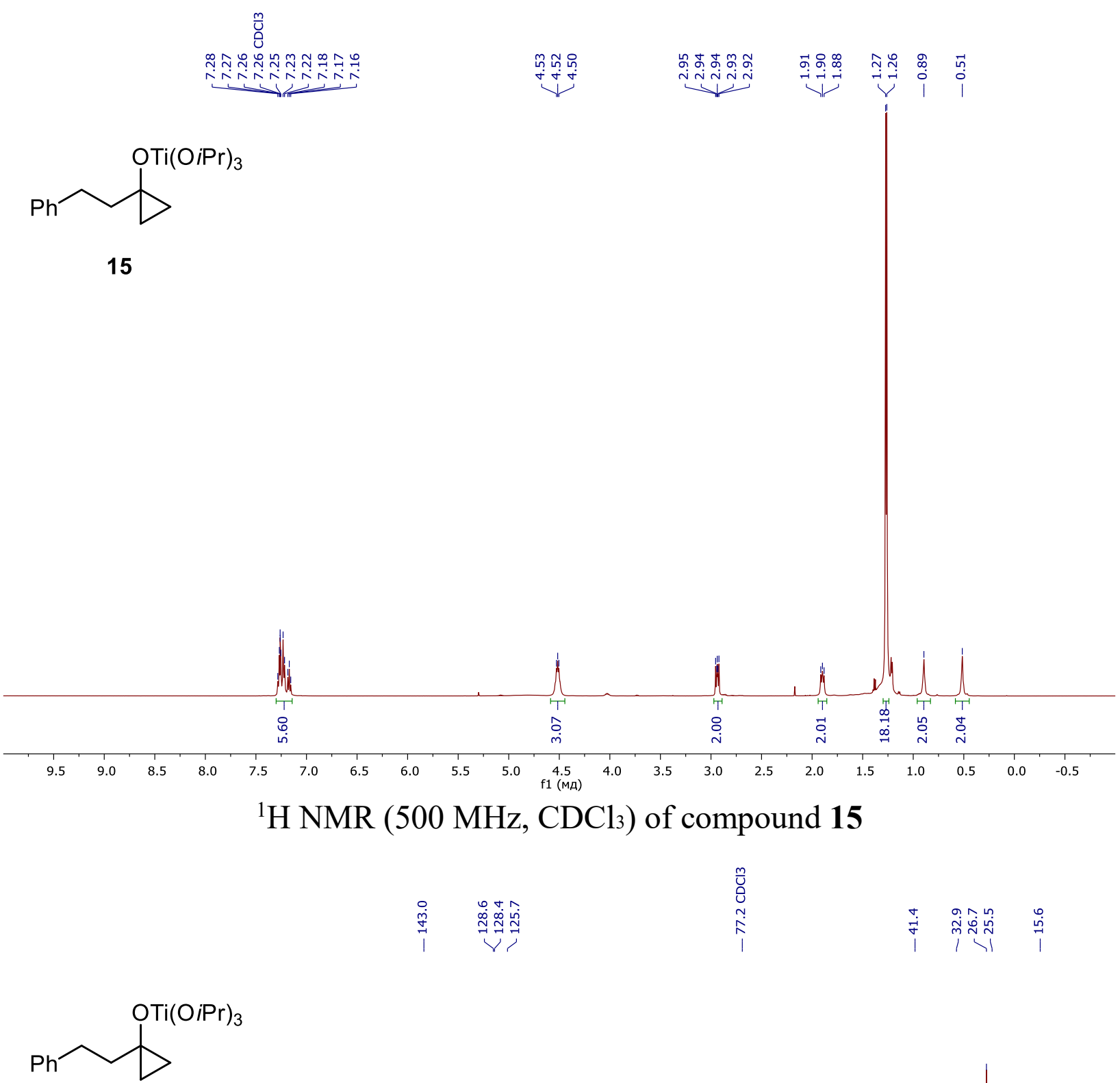

15

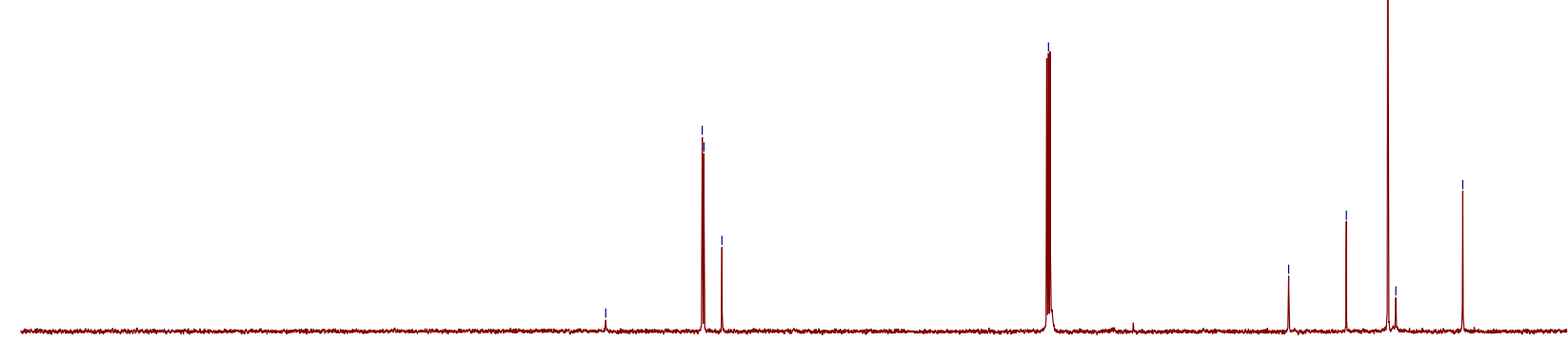

$\begin{array}{lllllllllllllllllllllllllll}30 & 220 & 210 & 200 & 190 & 180 & 170 & 160 & 150 & 140 & 130 & 120 & 110 & 100 & 90 & 80 & 70 & 60 & 50 & 40 & 30 & 20 & 10 & \end{array}$

${ }^{13} \mathrm{C}$ NMR (126 MHz, $\left.\mathrm{CDCl}_{3}\right)$ of compound 15 
